Universidad Politécnica de Cartagena

Escuela TéCnica Superior de Ingeniería Industrial

\title{
ANÁlisis, DECODIFICACIÓN Y CLASIFICACIÓN DE LA SEÑAL EEG EN ENTORNOS TRIDIMENSIONALES
}

Tesis presentada por Teodoro García Egea PARA OBTENER EL GRADo DE Doctor INGENIERo

Co-Directores: Dr. D. Juan López Coronado y Dr. D. José Luis Muñoz Lozano. Departamento de Ingeniería de Sistemas y Automática 


\section{Agradecimientos}

"Mientras el río corra, los montes hagan sombra y en el cielo haya estrellas, debe durar la memoria del beneficio recibido en la mente del hombre agradecido"

Virgilio

"Dicen que en la vida hay que escribir un libro, plantar un arbol y tener un hijo. Sin embargo, lo dificil es leer el libro, regar el arbol y criar al hijo"

Anónimo

Gracias...

A mi querida Teté, casi 10 años juntos y una niña preciosa. El futuro es desconocido, sin embargo de lo único de lo que estoy seguro es de querer vivirlo a tu lado.

A mi pequeña María José, que ha estado junto a mi en esas noches en las que escribía las últimas palabras de este trabajo.

A mi padre y a mi madre, que se han dejado la vida para enseñarnos el valor de lo importante a mis hermanos y a mí.

A mis suegros y cuñados, a quienes agradezco lo mejor que tiene mi familia, mi mujer.

A Juan López Coronado, mi director de tesis, mi amigo y alguien a quien siempre estaré agradecido. 
Agradecimientos

A José Luis Muñoz, porque aunque nunca me ha dado clase, siempre ha estado ahí para hacerme crecer como ingeniero. Gracias por tu dirección y por ayudarme en este trabajo revisando hasta la última coma.

A Pedro y Nuria que desde que los conocimos siempre han sido la perfecta definición de esos amigos de tercer grado de los que habla Ciceron en su "tratado de la amistad".

A Jose Luis, José, Samuel y Marco que me han ayudado a profundizar en la fe y mejorar como persona.

A Manolo, Ana, Benjamín, Jorge, Sofía, Ana Belén, Pedro, Jesús, Pablo, etc. Llevamos toda la vida compartiendo alegrias y penas, y este momento no iba a ser menos.

A Mario, Miguel y Francisco, porque me han acompañado y me acompañarán en todos los momentos importantes de mi vida, como los buenos amigos que son.

A Jesús, porque nuestra amistad se ha forjado surcando los mares, descendiendo montañas, en temperaturas bajo cero y en largos viajes en coche.

A Jesús, Pablo, Arsenio, Alfonso, Juan, etc. En quienes he encontrado una familia de acogida durante mis días de trabajo en Madrid.

A mis abuelos, que desde el cielo me ayudan en cada paso que doy. A mi abuela por su cariño incondicional hacia mí durante 30 años.

A Juan Manuel y Yoya, que nos acompañaron en el momento más importante y feliz de nuestras vidas.

A Vicente e Isabel, por abrirme siempre las puertas de vuestra casa y de vuestro corazón.

A Pablo e Isabel, porque son para nosotros un modelo a seguir como personas, como padres luchadores y como amigos.

A Cristina Nombela, la primera persona que me enseñó a colocar un electrodo electroencefalografico y con quien registré por primera vez una señal cerebral.

A mi amigo y compañero Pablo, gracias por tus consejos, tu tiempo, tu trabajo y tu amistad.

A mi colega y amigo Juanmi, porque trabajando en proyectos apasionantes hemos conseguido aumentar nuestra amistad. Espero que sigamos así durante mucho tiempo. 
A mi profesor, Decano y amigo Juan Luis Pedreño. Sus consejos siempre me han ayudado a mejorar personal y profesionalmente.

A Pablo por brindarme tu amistad y haberme introducido en el apasionante mundo del internet de las cosas.

A Fernando, porque fue de las primeras personas que confió en mi y me ayudó a crecer como Ingeniero y como persona. Espero seguir trabajando durante muchos años contigo.

A José Antonio y Rafael, porque desde que llegué a Madrid confiaron en mí para asumir importantes responsabilidades.

Gracias a Dios, por todas las buenas personas que ha puesto siempre en mi camino.

A los verdaderos amigos... 


\section{Índice general}

$\begin{array}{ll}\text { Agradecimientos } & 2\end{array}$

1. Introducción 1

1.1. Introducción . . . . . . . . . . . . . . . . . . . . 1

1.2. Alcance y objetivos . . . . . . . . . . . . . . . . 7

1.3. Desarrollo de la Investigación . . . . . . . . . . . . . . . . . . . 8

1.3.1. Análisis de las bases biológicas y estudio de los métodos de clasificación para señales cerebrales . . . . . . . . . . . . . 9

1.3.2. Obtención de datos y desarrollo del experimento . . . . . . . . 10

1.3.3. Transferencia tecnológica e integración con otros sistemas . . . 10

1.4. Organización de la Tesis . . . . . . . . . . . . . . . . . . . . 11

2. Fundamentos del Brain Computer Interface 13

2.1. Introducción . . . . . . . . . . . . . . . . . . . . . 13

2.1.1. El modelo Brain Computer Interface . . . . . . . . . . . . . 16

2.1.2. Evolución histórica del BCI . . . . . . . . . . . . . . . . 18

2.2. Bases del modelo BCI . . . . . . . . . . . . . . . . . . . . . . . 21

2.2.1. Bases biológicas y modelo neuronal . . . . . . . . . . . 23

2.2.2. La corteza cerebral y áreas de Brodmann . . . . . . . . . . . . 24

2.2.3. Relación estímulo-respuesta . . . . . . . . . . . . . . . . 33

2.3. Generación de la señal EEG . . . . . . . . . . . . . . . . . . . . 34

2.3.1. Ritmos cerebrales . . . . . . . . . . . . . . . 38

2.3.2. Conclusiones ... . . . . . . . . . . . . . . 44 
3. Análisis, procesado y clasificación de señales EEG 47

3.1. Introducción . . . . . . . . . . . . . . . . . . 47

3.2. Tipos de sistemas BCI . . . . . . . . . . . . . . . . . . . . 49

3.2.1. Sistemas sincronos y asincronos . . . . . . . . . . . . . 50

3.2.2. Sistemas endógenos y exógenos . . . . . . . . . . . . . 51

3.2.3. Dependientes e Independientes . . . . . . . . . . . . . . . 56

3.3. Análisis y clasificación de la señal en sistemas BCI . . . . . . . . . . . 57

3.3.1. Detección, selección y extracción de características . . . . . . . 58

3.3.2. Sistemas BCI espontáneos o de potenciales evocados . . . . . 64

3.3.3. Sistemas BCI online y offline . . . . . . . . . . . . . . 65

3.4. Algoritmos de Clasificación en sistemas BCI . . . . . . . . . . . . 67

3.4.1. Problemas en la clasificación en entornos BCI . . . . . . . . . 94

3.4.2. Comparativa de algoritmos BCI . . . . . . . . . . . . . 97

3.4.3. Algoritmos Genéticos . . . . . . . . . . . . . . . . 98

3.5. Conclusiones . . . . . . . . . . . . . . . . . . . 104

4. Decodificación y clasificación de la señal EEG en entornos tridimen$\begin{array}{lc}\text { sionales } & 109\end{array}$

4.1. Introducción . . . . . . . . . . . . . . . . . . . . . . 109

4.2. Materiales y métodos . . . . . . . . . . . . . . . . . . . . 113

4.2.1. Método de clasificación . . . . . . . . . . . . . . . 118

4.2.2. Selección de electrodos . . . . . . . . . . . . . . . . 121

4.3. Resultados . . . . . . . . . . . . . . . . . . 126

4.4. Discusión y conclusiones . . . . . . . . . . . . . . . . . 131

$\begin{array}{ll}\text { 5. Conclusiones } & 137\end{array}$

5.1. $\operatorname{Logros} \ldots \ldots \ldots$. . . . . . . . . . . . . . . 137

5.2. Desarrollos futuros . . . . . . . . . . . . . . . . . 139

5.3. Nuevo sistema basado en GoogleGlass y BCI . . . . . . . . . . . . . . 141

5.3.1. Actuadores en sistemas BCI . . . . . . . . . . . . . . . 143

5.3.2. GoogleGlass como actuador en sistemas BCI . . . . . . . . . . 150

5.3.3. EMOTIV EPOC y GoogleGlass . . . . . . . . . . . . . . . . 152

5.4. Modelo combinado BCI - GoogleGlass . . . . . . . . . . . . 157 
Bibliografía 


\section{Capítulo 1}

\section{Introducción}

" Tienes la cara del que acepta lo que ve, porque está esperando despertar"

Matrix

\subsection{Introducción}

La tecnología Brain Computer Interface o BCI está basada en la monitorización, el análisis y la decodificación de las señales generadas por las distintas estructuras cerebrales bajo determinadas condiciones. Un sistema BCI puede utilizar diversas técnicas para analizar la actividad cerebral con el objetivo de establecer un canal de comunicación directo entre el usuario y un dispositivo externo [1] [2]. Interpretando estos procesos y traduciendo esta información en órdenes concretas, será posible controlar sistemas software o hardware a partir de señales cerebrales, obtenidas tanto mediante tecnicas invasivas o no invasivas.

Un interfaz BCI no invasivo basa su funcionamiento en el análisis mediante métodos de baja invasividad. Uno de los métodos no invasivos más utilizados esta basado en la obtención de señales electroencefalográficas o EEG a través de electrodos colocados en la cabeza del sujeto. Estas señales serán captadas durante la presentación de estimulos al sujeto o bien durante algún tipo de actividad mental y/o motora. En algunas ocasiones, se lleva a cabo este procedimiento con el objetivo de extraer la correlación existente entre el estimulo que el sujeto recibe o la acción ejecutada por el 
sujeto y la respuesta cerebral generada. La actividad EEG incluye una amplia variedad de ritmos diferentes identificados por su frecuencia, localización o amplitud, que hacen que la señal EEG sea extremadamente compleja [3]. Sin embargo, numerosos estudios [4] [5] muestran que existe un enorme campo de actuación cuyo desarrollo permitirá identificar, clasificar y controlar algunas características de dicha actividad EEG. A pesar de que la tecnología BCI todavía está muy lejos de poder interpretar estados mentales complejos e identificar pensamientos específicos, a día de hoy es posible interpretar patrones cerebrales en las señales eléctricas de la corteza cerebral utilizando señales electroencefalográficas o EEG.

En los últimos años están surgiendo infinidad de productos electrónicos relacionados con la estimulación cerebral. Esta situación muestra que, lejos de encontrarse confinada en los laboratorios de investigación, la percepción, agilidad y capacidad mental es algo que preocupa cada vez más al ciudadano de a pie [6]. Esta preocupación, junto con el creciente aumento de enfermedades degenerativas consecuencia de una mayor esperanza de vida en el mundo desarrollado, hace del trabajo con electroencefalografía y neurofeedback una pieza clave para el desarrollo humano. Los sistemas BCI tienen aplicaciones en muchos campos de la industria, la asistencia sanitaria e incluso el ocio [7]. Por ejemplo, sería posible utilizar un sistema BCI para construir una palabra completa, a través de la selección de cada una de las letras que componen dicha palabra [8], es posible calibrar el sistema para que se adapte a las señales EEG del sujeto. Una vez el sistema es capaz de identificar con cierta precisión los cambios en la actividad cerebral tras una serie de sesiones de calibración, el usuario está listo para escribir palabras utilizando únicamente su cerebro [9].

Existen gran cantidad de estudios [10] [11] [12] que demuestran que los diferentes procesos cerebrales pueden ser identificados mediante el análisis de los trazados electroencefalográficos. Estas señales EEG están directamente relacionadas con la actividad funcional del cerebro y pueden ser identificados y comparados. Por tanto, será posible desarrollar un mecanismo que controle la aparición de dichos estándares de actividad cerebral asociados a acciones o estimulos concretos. El nivel de complejidad del proceso de decodificación y clasificación de la señal electroenfalográfica está directamente relacionado con el tipo de acción asociada.

Algunos autores [13] [14] han sido capaces de decodificar la señal electroence- 


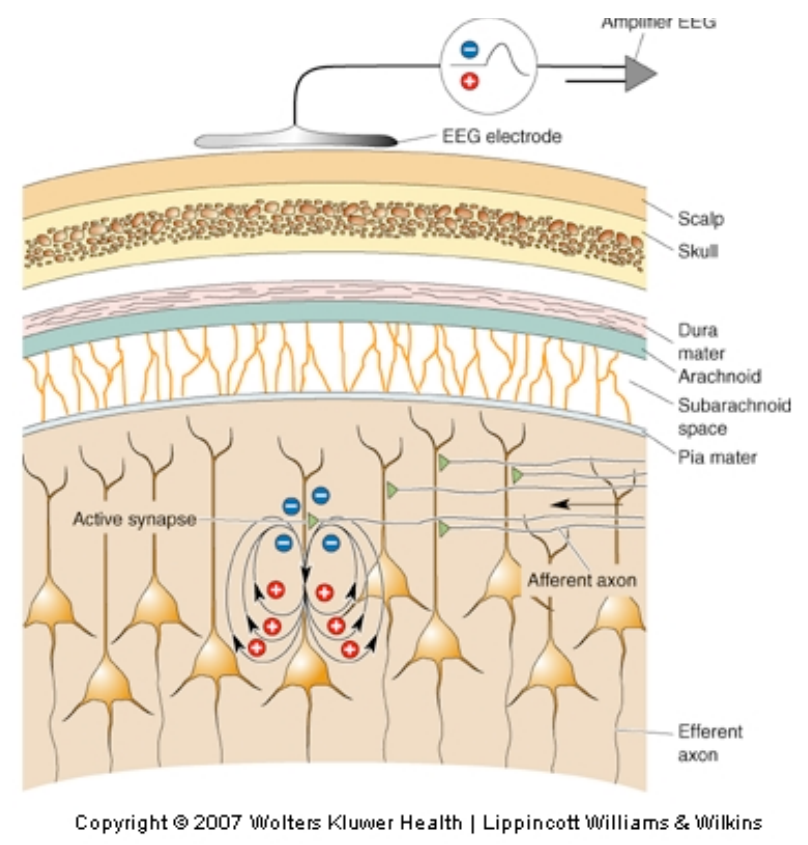

Figura 1.1: Captación de la actividad cerebral mediante un electrodo EEG

falográfica asociada a movimientos sencillos o decisiones con pocas opciones, desarrollándose sistemas capaces de interpretar esa señal cerebral y traducirla a acciones concretas.

La adquisición de la señal cerebral puede realizarse utilizando distintos métodos que pueden clasificarse en dos grandes grupos en función de la utilización de métodos invasivos o no invasivos. Algunos autores han llevado a cabo con éxito experimentos mediante técnicas como la magnetoencefalografía (MEG) [15] [16] la Electrocorticografía (ECoG) [17] [18], o la resonancia magnética funcional (fMRI) [19] [20]. Determinadas técnicas invasivas como el electrocorticograma presenta evidentes ventajas respecto de la señal EEG por su precisión y menor ruido de la señal obtenida. Sin embargo las técnicas invasivas generan un gran riesgo al paciente [21] [22] [23], lo que supone una desventaja importante para su utilización de forma masiva. A pesar de estos riesgos, muchos experimentos demuestran que con estos sistemas invasivos es posible obtener una alta precisión en la decodificación y clasificación de la señal cerebral [24]. Existen también otros métodos no invasivos, ya mencionados anteriormente, como la resonancia magnética funcional (fMRI), el magnetoencefalograma (MEG), etc. Estas tecnologías poseen características que desaconsejan su uso para 


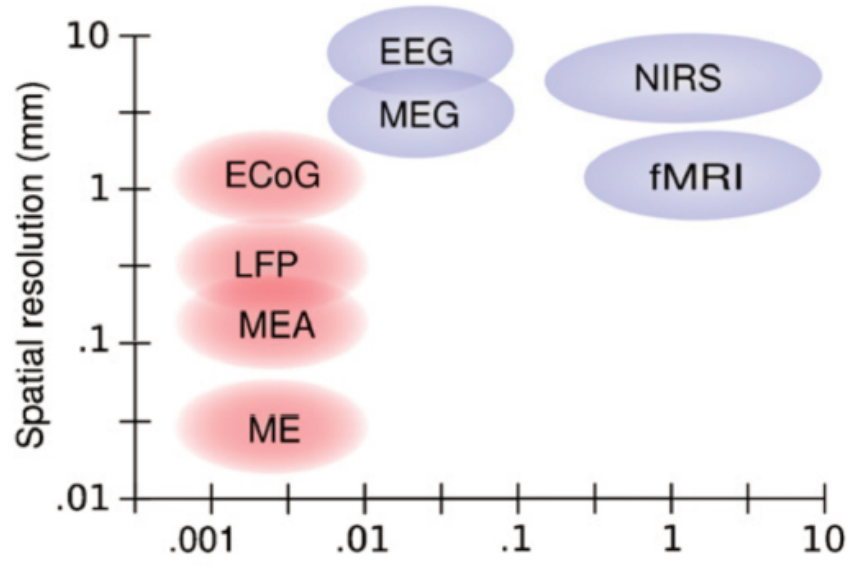

Figura 1.2: Comparativa entre métodos de adquisición de señal cerebral. Resolución espacial y temporal de los distintos metodos de obtención de la señal cerebral, tanto invasivos como no invasivos [28]

investigaciones en el campo del BCI debido a su complejidad, su alto precio y el hecho de que no sean adecuadas para su uso durante largo tiempo. Además, en el caso de la fMRI, cabe destacar como desventaja evidente, su baja resolución temporal (Figura 1.2). Este hecho hace que este tipo de métodos no invasivos tengan especial relevancia en aquellos estudios en los que se han utilizado conjuntamente con sistemas EEG [25] [26].

A pesar de lo anterior no se deben descartar totalmente los experimentos realizados a partir de técnicas invasivas, ya que dichos experimentos aportan información útil que puede ser utilizada posteriormente en otro tipo de ensayos [27]. El potencial de las técnicas invasivas está liderado por el electrocorticograma (ECoG) y los arrays de micro electrodos, que han sido utilizados en experimentos BCI arrojando resultados muy positivos en términos de precisión en la decodificación de las señales obtenidas [17] . Sin embargo, este tipo de tecnología presenta una serie de desventajas más allá de su invasividad. El ECoG se encuentra limitado debido a que una vez colocadas es muy dificil cambiar su ubicación. Además ofrecen desventajas adicionales ya que con este tipo de técnicas frecuentemente no es posible cubrir la totalidad del cortex. En la Figura 1.2 se realiza una comparativa entre los distintos métodos de adquisición de la señal cerebral en función de su invasividad y su resolución temporal. 
En el presente trabajo se pretende demostrar que los sistemas no invasivos basados en EEG pueden mejorar la precisión en la decodificación, obteniendo resultados comparables e incluso superiores a los alcanzados por sistemas BCI invasivos. Si el desarrollo del BCI basado en señales EEG consigue precisiones en la decodificación de la señal cerebral superiores a las obtenidas utilizando técnicas invasivas, se estará dando un paso importante para implantar los sistemas BCI.

Actualmente la robótica y la neurociencia poseen un gran potencial de desarrollo a través de la Neuro-robótica. Este campo representa un reto tecnológico y científico en el que toman parte disciplinas procedentes de la ingeniería y la medicina con un objetivo compartido: profundizar en el conocimiento de los procesos neuronales y neuromotores que dan lugar a determinados comportamientos, trasladando dichos conocimientos a la neuro-robótica. Un sistema BCI típico posee una señal de entrada, una señal de salida y un mecanismo complejo de procesado de señal que une la entrada con la salida (Figura 1.3). Un sistema BCI permite tener acceso directo en tiempo real a la información sobre la actividad cerebral del usuario. Para mejorar el proceso de clasificación y decodificación de la señal, existe la posibilidad de aplicar algoritmos genéticos con el objetivo de seleccionar aquellas características de la señal que contienen mayor información útil para el proceso de clasificación. En este sentido, algunos autores [29] han demostrado la efectividad de utilizar algoritmos genéticos a la hora de reducir el número de electrodos en experimentos BCI y aumentar sus prestaciones.

La evolución de la neurotecnología pasa por el análisis del conjunto de las funciones cerebrales asociadas no solo a procesos cognitivos, sino también al sistema motor. Esta línea de actuación abre nuevas oportunidades de colaboración entre científicos, neurocientíficos e ingenieros. Todo ello va a permitir arrojar luz sobre determinados comportamientos, que son fruto de procesos enormemente complejos y dinámicos tales como el aprendizaje continuo, la mejora de habilidades motoras o desarrollo cognitivo. La robótica está lista para proveer a cualquier disciplina de componentes antropomorfos que permitan validar las tesis planteadas por la neurociencia y la neurotecnología. El camino que queda todavía por recorrer debe mejorar la interacción entre la robótica y el ser humano a través de modelos flexibles y adaptativos, con los nuevos paradigmas basados en BCI [31]. La interacción entre neurociencia e ingenie- 


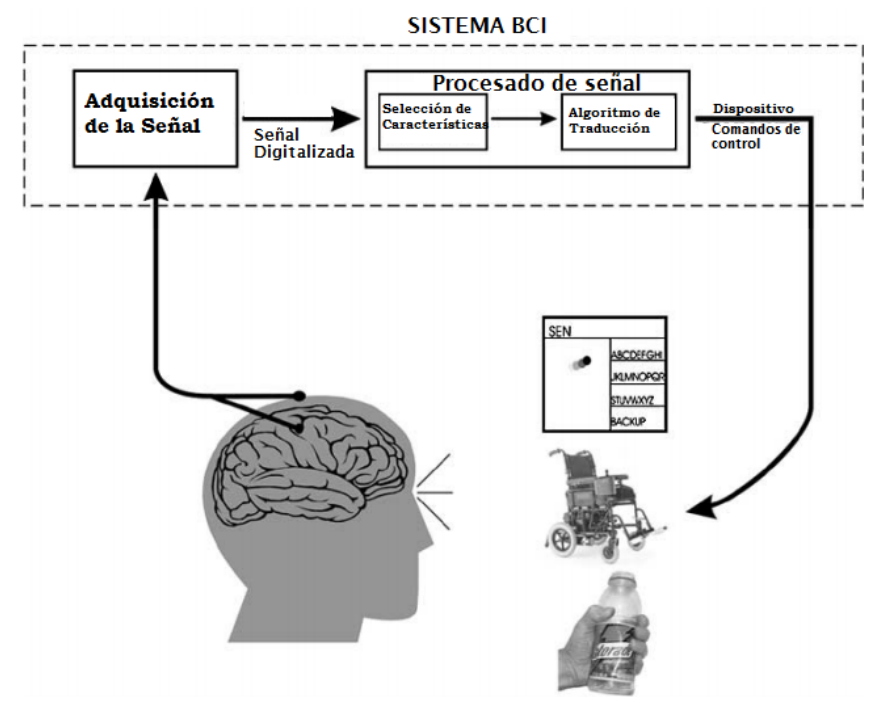

Figura 1.3: Esquema general de un sistema BCI [30]

ria permitirá mejorar el diseño de los nuevos dispositivos antropomorfos y mejorar el rendimiento de los sistemas BCI. Estos sistemas poseen además un enorme potencial si los combinamos con sistemas robóticos antropomorfos. Este extremo se aborda también en siguientes capítulos, todo ello desde la colaboración multidisciplinar al servicio del desarrollo de nuevos productos neurotecnológicos integrados. Desde que algunos investigadores comenzaron a explorar el uso de dispositivos robóticos para la restitución de movimientos, han existido progresos importantes [32] pero se ha mantenido la idea de que la comercialización y uso de este tipo de dispositivos estaba más cercano a la ciencia ficción que a la realidad. Aunque todavía hoy existen retos asociados con el diseño robótico que deben ser perfeccionados, los avances en este campo han sido enormes [33]. La combinación de la robótica y los sistemas BCI han arrojado buenos resultados en recientes estudios, que permiten atisbar un futuro esperanzador para sujetos con movilidad reducida [34].

El presente trabajo está centrado en el análisis del movimiento de las extremidades superiores del sujeto. El sujeto realizará una serie de trayectorias con su brazo, en un entorno tridimensional mientras se adquiere la señal EEG generada durante la ejecución de la tarea. Esto permitirá analizar la intencionalidad motora del sujeto y evaluar las posibilidades de los sistemas BCI no invasivos para obtener altas 


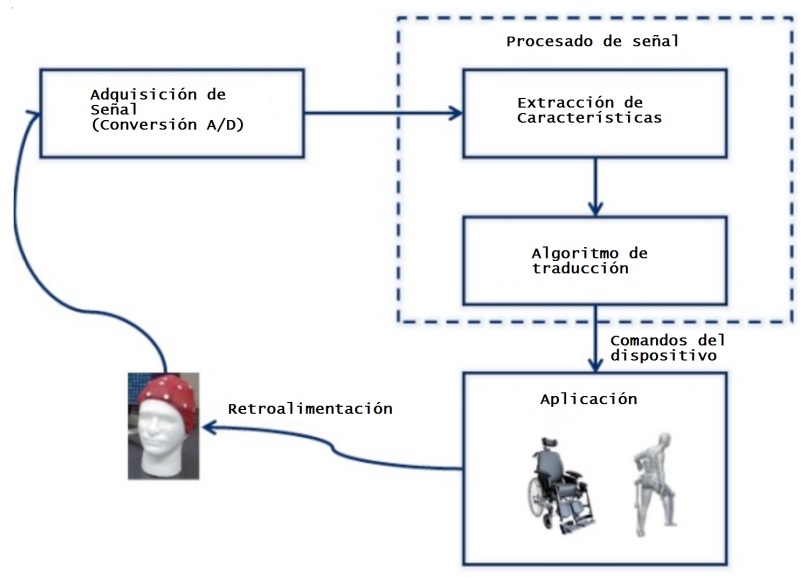

Figura 1.4: Ejemplo de un sistema BCI para restitución del movimiento en extremidades inferiores

precisiones en la decodificación en entornos tridimensionales.

Esta tesis doctoral se enmarca en el contexto general descrito hasta ahora. Los objetivos del presente trabajo están en línea con parte de los objetivos propuestos en proyectos de investigación desarrollados por el grupo de investigación NEUROCOR del que el autor de la presente Tesis forma parte. Los proyectos han sido financiados a nivel nacional e internacional en convocatorias competitivas. En este punto merecen especial mención los proyectos INCAMFUT e IRENE, relacionados directamente con el análisis, procesado, decodificación y clasificación de la señal cerebral para aplicaciones en sistemas BCI o el proyecto Exo-LEGS.

\subsection{Alcance y objetivos}

Este trabajo tiene como objetivo demostrar que es posible obtener un alto nivel de precisión en la decodificación de la señal electroencefalográfica, durante la realización de experimentos complejos en entornos tridimensionales. Con un adecuado proceso de adquisición, procesado, entrenamiento y clasificación de la señal electroencefalográfica, será posible obtener información suficiente a fin de clasificar movimientos y trayectorias en entornos tridimensionales con un alto nivel de precisión.

Los objetivos principales de esta Tesis Doctoral son los siguientes: 
1. Desarrollar un sistema BCI basado en tecnologías no invasivas, capaz de decodificar la intencionalidad del sujeto a través de la determinación de trayectorias en entornos tridimensionales.

2. Demostrar que es posible obtener altas precisiones en la decodificación de la señal electroencefalográfica en sistemas BCI.

3. Desarrollar un nuevo modelo de BCI que permita añadir robustez y nuevas funcionalidades al modelo propuesto

\subsection{Desarrollo de la Investigación}

Este trabajo pretende refrendar las teorías desarrolladas respecto de la señal EEG y las posibilidades futuras del BCI con experimentos que certifiquen su validez. En primer lugar se realizarán las observaciones pertinentes que permitan, a través de la inducción, extraer el principio particular de cada una de dichas observaciones. Paralelamente se plantearán las hipótesis que corresponda en cada caso para posteriormente confirmar dicho planteamiento mediante la ejecución del experimento. En base al resultado obtenido se extraerán las conclusiones correspondientes. Las teorías extraídas del presente trabajo, estarán apoyadas en experimentos científicos bajo condiciones parametrizadas que certifican su validez.

En primer lugar se han diseñado experimentos de adquisición de señal EEG para su posterior análisis. En colaboración con el Kinesiology laboratory de la "School of Public Health" de la Universidad de Maryland (EEUU), han sido seleccionados seis sujetos para realizar una tarea de movimiento de una extremidad superior desde un punto central hasta ocho puntos diferentes colocados alrededor de dicho punto central inicial y en diferentes planos. Durante el movimiento de cada uno de los sujetos, se obtienen datos respecto de la trayectoria espacial que realiza, junto con la señal EEG que genera el sujeto durante el intervalo en el que su extremidad se desplaza. El experimento ha sido diseñado de tal forma que permita disponer de la mayor cantidad de información posible, tanto desde el punto de vista de la señal EEG generada como respecto del movimiento efectuado por brazo. En este experimento toman parte varios sujetos que realizan sucesivos intentos hacia unos objetivos o 
"targets" situados de una forma concreta en un entorno tridimensional.

Entre los objetivos también está la evaluación de los diferentes algoritmos utilizados en entornos BCI. Se aborda la posibilidad de explorar nuevas formas de aplicación de los sistemas interfaz cerebro máquina para potenciar las prestaciones de dichos sistemas en personas con movilidad reducida o minusvalía severa. Además, se planteará la utilización de la información disponible a través del análisis de la señal electroencefalográfica junto con otra información de tipo posicional o inercial de los objetos del entorno, todo ello con el objetivo de mejorar el nivel de precisión de los sistemas BCI. Se desarrollará software específico para permitir al usuario visualizar la actividad cerebral e interaccionar a traves de las GoogleGlass [35]. Este punto merece especial atención, ya que el establecimiento de un canal de comunicación entre las googleGlass y un dispositivo BCI, puede abrir infinidad de posibilidades para el desarrollo de estos sistemas. Estas nuevas funcionalidades de los sistemas BCI pueden complementar a los sistemas robóticos antropomorfos controlados por BCI no invasivo, convirtiéndose en una pieza clave en el campo de la robótica asistencial.

\subsubsection{Análisis de las bases biológicas y estudio de los méto- dos de clasificación para señales cerebrales}

Se debe tener en cuenta el contexto bajo el que se realiza la adquisición de la señal cerebral para que, una vez tratada y analizada, pueda ser clasificada con la mayor precisión. Por tanto, será necesario conocer los mecanismos biológicos y fisiológicos que desencadenan los potenciales de acción, así como el modelo que opera entre las distintas estructuras cerebrales. En ese sentido, es de vital importancia conocer la forma en la que las estructuras cerebrales codifican la información sobre determinadas acciones del sujeto en señales cerebrales que se reflejan en la corteza cerebral y que serán captadas y analizadas por un sistema BCI.

Conocer los mecanismos de generación de la señal cerebral es importante para realizar el proceso inverso de decodificación. Conocida la señal reflejada en la corteza cerebral y captada a través de electrodos EEG, ésta debe ser procesada y clasificada mediante aquel algoritmo o conjunto de algoritmos que minimicen el error a la hora de obtener el proceso motor o cognitivo que generó esta señal. 


\subsubsection{Obtención de datos y desarrollo del experimento}

La obtención de las señales cerebrales se realizará mediante sistemas de monitorización EEG no invasivos cuyas caracteristicas serán descritas posteriormente. Los conjuntos de datos se obtendrán a partir de un número suficiente de sujetos seleccionados que desplazarán una extremidad superior en desde un punto central hasta ocho puntos diferentes, colocados tal y como se ha descrito en 1.3. Cada sujeto realizará un número de intentos suficiente durante los cuales el brazo sigue la trayectoria desde el punto central a cada uno de los targets. Durante el desplazamiento, se obtendrán datos de la trayectoria espacial que realiza. Toda esta información sera recopilada junto con la señal EEG que genera el sujeto, no sólo a lo largo del intervalo en el que su extremidad se desplaza, sino también unos segundos antes de comenzar el movimiento.

\subsubsection{Transferencia tecnológica e integración con otros sis- temas}

Esta tesis doctoral está centrada en la decodificación de las señales cerebrales que originan y guían la ejecución del movimiento de una extremidad superior en un entorno tridimensional. Tal y como se expondrá en siguientes capítulos, conseguir altas precisiones en la decodificación de las señales electroencefalográficas durante este tipo de tareas, supone un paso importante a la hora de desarrollar extremidades robóticas, capaces de sustituir a las extremidades humanas y recibir órdenes directamente de la corteza cerebral, todo ello utilizando métodos no invasivos de captación de la señal. El siguiente gran paso del BCI debe hacerse combinando las prestaciones que ya hoy ofrecen los sistemas BCI, con los últimos desarrollos en el campo de la robótica y, con sistemas de visión asistida. Los dispositivos de asistencia que se comenzarán a desarrollar, implementar y comercializar en un futuro próximo estarán formados por sistemas BCI de análisis de la señal cerebral en combinación con otros sistemas robóticos y de análisis del entorno. Estos sistemas tendrán la posibilidad de adaptarse a las características particulares de cada situación y cada sujeto, todo ello a través de algoritmos inteligentes que analizarán determinados parámetros durante un periodo de aprendizaje inicial adaptando su funcionamiento futuro. Esto 
permitirá optimizar la precisión de estos sistemas, mejorar la adaptación a las características particulares de cada sujeto y mejorar la toma de decisiones. La utilización de algoritmos genéticos permitirá optimizar la operación global del sistema mediante la selección de aquellos electrodos que aportan información útil y no redundante.

El presente trabajo pretende mostrar el gran número de posibilidades que se abren con el desarrollo conjunto del BCI, la robótica y la visión por computador no solo dentro del laboratorio, sino también fuera de él. El desarrollo de un nuevo modelo de BCI portatil que sirva como base de desarrollos futuros mas complejos, también forma parte del presente trabajo.

\subsection{Organización de la Tesis}

Este trabajo se estructura en 5 capítulos que desarrollan los objetivos anteriormente expuestos.

En el segundo capítulo se describen las principales características de un sistema BCI en función del contexto en el que se desarrolla el experimento. En este apartado también se aborda la evolución histórica del BCI así como otros aspectos sobre la composición y tratamiento de la señal EEG. Además, se describen los fundamentos biológicos y neurológicos responsables de la generación de la señal cerebral asociada a procesos motores o cognitivos.

En el tercer capítulo se describe el estado del arte de los principales algoritmos utilizados en los experimentos BCI para decodificar, analizar y clasificar la señal EEG. Se analizan y describen las principales características de cada uno de ellos, dependiendo del tipo de datos analizado. Este capítulo se centra en una de las partes clave de cualquier sistema BCI, ya que los algoritmos utilizados condicionarán, en gran medida, los resultados del experimento en términos de precisión en la clasificación, agilidad del proceso de decodificación, coste computacional, etc.

En el cuarto capítulo, se muestran los resultados de decodificación de señal obtenidos en un experimento BCI mediante técnicas no invasivas, en un entorno tridimensional. En este apartado se describe detalladamente el modelo BCI completo desde la adquisición de la señal EEG, durante la realización de una determinada tarea por parte de los sujetos, pasando por el procesado y clasificación de dicha señal, junto 
con el análisis de los resultados de todo el proceso. La comparación de los resultados obtenidos permitirá extraer conclusiones sobre el avance que ha supuesto este estudio en relación con otros experimentos realizados con tecnicas invasivas. Además, se discuten los resultados obtenidos tras aplicar algoritmos genéticos a los análisis de la señal EEG permitiendo seleccionar aquellos canales que más información transportan, reduciendo así las dimensiones de la información a tratar y, en consecuencia, aumentando la velocidad de ejecución del proceso completo. E

En el quinto capítulo se describen los principales avances y conclusiones derivadas del trabajo descrito en los capítulos anteriores. n este capítulo, también se desarrolla un nuevo modelo de BCI portátil concebido en el marco de la presente tesis doctoral. Este nuevo modelo de BCI esta integrado por dispositivos de última generación que añaden robustez y nuevas funcionallidades al modelo desarrollado. Con ese punto de partida, se describen algunas líneas futuras en el marco del BCI y su interacción con la robótica en línea con los avances aquí expuestos. Estos retos podran ser desarrollados e implementados tomando como base el trabajo realizado en esta tesis doctoral. 


\section{Capítulo 2}

\section{Fundamentos del Brain Computer Interface}

Ahora sé por qué llorán los humanos, pero eso es algo que yo nunca podré hacer

Terminator

\subsection{Introducción}

El Brain Computer Interface puede definirse como un enlace directo entre el cerebro humano y un sistema externo, que detecta determinados patrones en la actividad cerebral y es capaz de traducir dichas señales en comandos para controlar un dispositivo externo. Las aplicaciones basadas en BCI nacieron con el objetivo de establecer nuevos canales de comunicación para aquellos usuarios con pérdida de movilidad o afectados por enfermedades como esclerosis lateral amiotrófica, tetraplegia, etc [36] [37]. El desarrollo de los sistemas BCI están suponiendo un cambio de paradigma desde el punto de vista de la interacción del ser humano con un teclado, ratón u otro tipo de dispositivo tal y como se ha venido demostrando desde los primeros experimentos exitosos con sistemas BCI [38]. Estos sistemas no sólo pueden ser complementarios a los dispositivos clásicos de control e interacción, sino que en determinados casos pueden llegar a sustituir total o parcialmente a estos tras un proceso de aprendizaje específico [39]. 
El sistema BCI establece un canal de comunicación independiente del camino que siguen los impulsos nerviosos desde el cerebro hasta los nervios y músculos periféricos [40]. Para conseguir controlar un dispositivo a través de la actividad mental generada por el usuario, los sistemas BCI utilizan diversas técnicas para diferenciar entre las diferentes actividades mentales. Dependiendo de las caracteristicas, los sistemas BCI pueden ser clasificados en función de diversos parámetros en: dependientes e independientes, síncronos o asíncronos, en función del fenómeno neurológico en el que se basa el sistema BCI, dependiendo del método de extracción, selección y traducción de características, e incluso en función del dispositivo de salida. Estas características del sistema BCI son sólo una pequeña muestra de la lista propuesta por algunos autores [41] que ha sido utilizada para describir el estado del arte de la tecnología BCI [42].

Si analizamos la naturaleza de las señales en las que se basa un sistema BCI, este trabajo y la mayor parte de trabajos de investigación realizados en los últimos años sobre tecnología BCI han tenido como base las señales EEG [43] [44] [45] [46]. Esto se debe a que este tipo de señales pueden ser captadas sin riesgo para el sujeto, de una forma no invasiva y conservando la correspondencia existente entre la información contenida en la señal EEG y los procesos cerebrales que la desencadenan [47] [48]. Sin embargo, también existen otras razones ligadas a las características propias de la señal EEG tales como la alta resolución temporal, la facilidad de uso o el relativo bajo coste de los componentes básicos para electroencefalografía. A lo largo de este capítulo se analizarán las bases biológicas, fisiológicas y computacionales que sustentan el funcionamiento de todo sistema BCI basado en señales EEG.

Los sistemas BCI poseen una estructura y modelo muy similar en todos los casos [30], aunque pueden diferir en el método mediante el que se obtiene la señal cerebral, el número de electrodos utilizados o el fin para el que fueron diseñados. El sistema detecta la presencia de patrones específicos en la actividad cerebral del sujeto. Una vez captada y analizada la señal, el sistema BCI es capaz de decodificar estos patrones contenidos en la señal, traduciéndolos en comandos de control. Para detectarlos, los sistemas BCI utilizan diferentes algoritmos de procesado de señal dependiendo del entorno en el que se desarrolla el experimento, tipo de tarea a ejecutar, naturaleza de la señal adquirida, dispositivo de salida o dimensiones de los datos obtenidos [49]. 
Estos algoritmos constituyen una parte fundamental del diseño de un sistema Brain Computer Interface. Todo ello puede apreciarse con detalle en la Figura 2.1. Además, en algunos sistemas, el usuario puede recibir información adicional a través de un display que muestra las señales generadas por el sistema BCI relativas a su actividad cerebral [50].

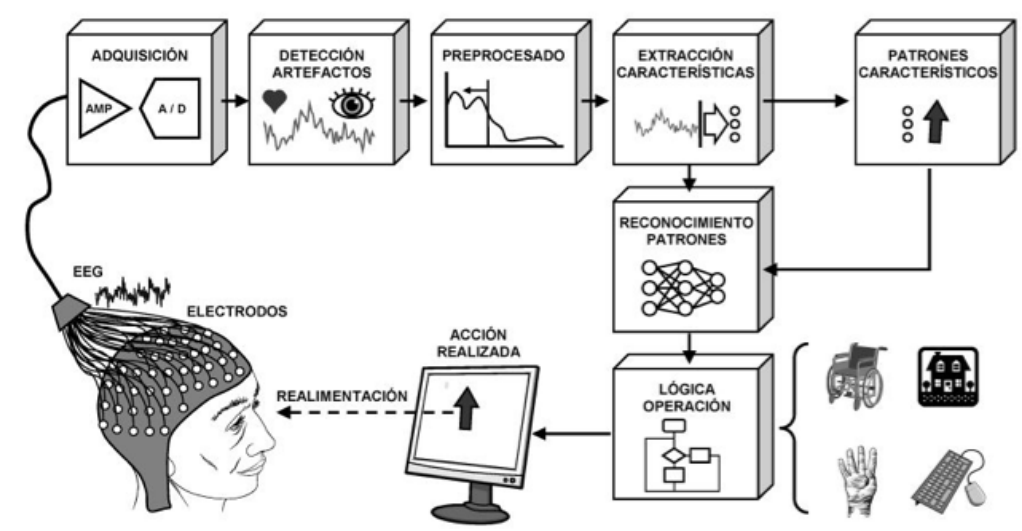

Figura 2.1: Diseño y funcionamiento general de un sistema BCI básico [49]

La señal cerebral captada a través de sistemas EEG no sólo contiene información útil para controlar un sistema BCI, sino que también contiene otro tipo de información procedente de fuentes distintas a la actividad cerebral. Este tipo de perturbaciones reflejadas en la señal EEG, cuyo origen no son las funciones cerebrales objeto de monitorización, se denominan 'artifacts'. Debido a esto, existen métodos [51] [47] que tienen como objetivo eliminar el ruido de la señal EEG separando la señal útil de los 'artifacts'. Este tipo de perturbaciones contenidas en la señal EEG no tienen por qué ser totalmente externas, sino que pueden proceder del propio sujeto pero de fuentes distintas a la actividad cerebral. Esto ocurre en el caso de las perturbaciones fisiológicas, producidas por movimientos voluntarios o involuntarios de la musculatura, tanto en la cara como en otras partes del cuerpo. La Figura 2.1 muestra el proceso que sufre la señal desde que es captada hasta su clasificación. Esta señal EEG también puede contener perturbaciones externas o extrafisiológicas como las procedentes de fuentes externas y ajenas al propio sujeto: perturbaciones electromagnéticas del ambiente, señales eléctricas de equipamientos cercanos, etc. Es necesario disponer de un subsistema que elimine el ruido procedente de otras fuentes 
distintas de la actividad cerebral que se encuentre contenido en la señal EEG antes de que esta señal sea amplificada o tratada. Algunos dispositivos de adquisición de señal EEG modernos poseen electrodos activos que permiten minimizar la aparición de perturbaciones extrafisiológicas en la señal EEG [52].

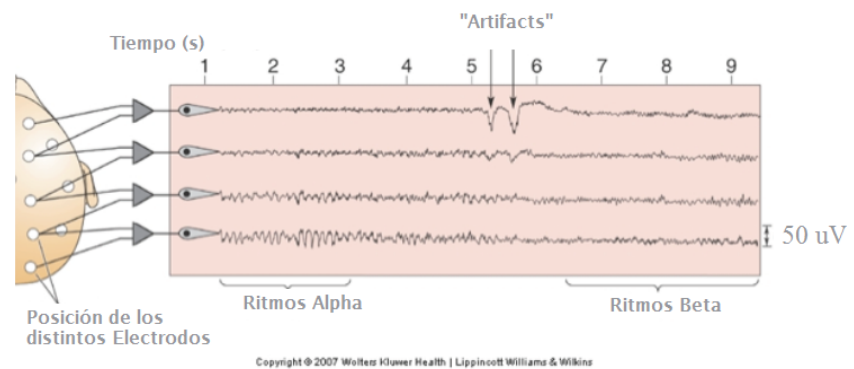

Figura 2.2: Registro de señales EEG afectadas por perturbaciones o ártifacts'

Para maximizar la precisión del sistema BCI decodificando el mecanismo neurológico que dio lugar a la señal original se prondrán en marcha algoritmos para reducir las dimensiones de la señal, facilitar su clasificación y extraer sus características principales. Este se describe en los bloques centrales de la Figura 2.1. En ese punto, las señales con demasiadas dimensiones o excesivo ruido son tratadas o descartadas. Sólo en sistemas ideales serán identificadas y seleccionadas aquellas características, dimensiones y canales que poseen significado y utilidad, en detrimento de aquellas señales que no aportan información útil al proceso de clasificación. En este proceso es posible introducir procesamiento previo. Utilizando algoritmos genéticos [53], se obtendrá el conjunto óptimo de electodos que aporten mayor cantidad de información útil, minimizando el error y optimizando la precisión del sistema BCI.

\subsection{1. $\quad$ El modelo Brain Computer Interface}

El modelo funcional y la estructura de un sistema BCI ha sido detalladamente descrito en el pasado por diversos autores [43] [49]. Es posible comprobar cómo algunos trabajos científicos combinan la tecnología BCI con la robótica para neurorehabilitación [54], permitiendo mejorar la calidad de la rehabilitación y disminuyendo el tiempo de recuperación. Las señales de entrada son procesadas extrayendo las características seleccionadas. Tomando como base las características seleccionadas, 
tiene lugar el proceso de clasificación, generando la señal de salida, cuyo destino final será el dispositivo o aplicación que ejecute la acción. El modelo BCI tiene el dispositivo de salida como su último eslabón. Los dispositivos de salida se han desarrollado gracias a la utilización de entornos de realidad virtual en los que el sujeto puede operar en entornos muy similares a los videojuegos [55] o ejercer el control de navegadores web [56] [57] a través del sistema BCI.

Los electrodos situados en la superficie de la cabeza del sujeto monitorizan la actividad cerebral obteniendo señales procedentes de la corteza cerebral, almacenando estas señales eléctricas que pueden ser posteriormente tratadas, filtradas, decodificadas, procesadas y clasificadas. Un sistema BCI se compone a su vez de varios subsistemas encargados de realizar tareas concretas. Las tareas desarrolladas por estos subsistemas dependen fundamentalmente de la naturaleza de la señal, de su procedencia, de sus características y del contexto en el que estas señales han sido obtenidas. La estructura de estos subsistemas no varía demasiado de un sistema BCI a otro, a pesar de que pudieran estar concebidos para fines muy distintos.

Dentro de las posibilidades para adquirir señales EEG disponibles en la actualidad, es dificil conseguir cierta calidad en la señal sin la utilización de fluidos o geles entre el electrodo y la superficie de la cabeza. Estos fluidos permiten reduzcir la impedancia y aumentar la conductividad. Sin embargo, desde la industria de los sistemas BCI se están dando los pasos necesarios para mejorar la tecnología y conseguir electrodos "secos" que consigan una calidad de señal similar a los electrodos "húmedos". Ya hoy existen algunos experimentos que utilizan electrodos secos en sistemas BCI con resultados esperanzadores [58]. Los electrodos secos no son utilizados todavía para experimentos BCI complejos, aunque hay estudios que han arrojado buenos resultados con este tipo de captadores de señal EEG. Algunos autores han desarrollado ensayos con electrodo seco [59] con buenos resultados tras un periodo inicial de 15 minutos de calibración y puesta en marcha. Estos resultados podrían ser el inicio de los futuros sistemas BCI para aquellas aplicaciones que requieran la monitorización continua de señales EEG durante largo tiempo. 


\subsubsection{Evolución histórica del BCI}

El Brain Computer Interface es una disciplina de investigación que aglutina importantes áreas no sólo de la ingeniería y la robótica sino también de la medicina y la neurociencia. Las investigaciones en este campo son relativamente recientes ya que los primeros experimentos que iniciarían el camino hacia el BCI fueron llevados a cabo por Carlo Matteucci (1811-1868) y Emil Du Bois-Reymond (1818 - 1896). Estos experimentos han sido descritos en la literatura científica [60] haciendo constar que fueron los primeros en establecer el concepto de neurofisiología al registrar señales eléctricas procedentes del movimiento muscular utilizando un galvanómetro. Sin embargo, no fue este el paso definitivo hacia lo que originaría la monitorización de señales electroencefalográficas y el Brain Computer Interface. Estos primeros experimentos en los que se registró por primera vez la señal cerebral procedente de movimientos musculares pueden considerarse una primera aproximación al análisis de las señales eléctricas del cerebro junto con los científicos Fritsch $(1838$ - 1927) y Hitzig $(1838$ - 1907) que fueron los primeros en llevar a cabo un experimento de estimulación eléctrica del cerebro. Algunos autores [61] atribuyen al fisiólogo inglés Catón el hecho de ser el primero en registrar corrientes cerebrales mientras examinaba cerebros de mono y conejo recogiendo las oscilaciones en un galvanómetro, observando que la iluminación que recibía el ojo de los animales era capaz de modificar el potencial de la señal obtenida. Este hecho fue posteriormente confirmado por Fleisch von Marxow en 1880, pudiendo comprobar además la facilidad de trabajar sobre la corteza cerebral a la hora de obtener registros. Con estos antecedentes, la comunidad científica atribuye a Hans Berger (1873 - 1941) la colocación de la primera piedra en el análisis de la señal cerebral tal y como hoy lo conocemos. Se le atribuye el descubrimiento de la existencia de las señales EEG en humanos registrando la actividad cerebral de un joven de 17 años. Comenzó sus estudios en este campo en 1902 con una motivación totalmente distinta que la de encontrar señales cerebrales para su monitorización; a estos estudios sobre la señal EEG dedicaría toda su vida [62]. Hans Berger es una referencia para todo aquel que investiga aspectos relacionados con la electroencefalografía. Su primer trabajo publicado sobre EEG en humanos data de 1929 y puede ser considerado uno de los padres de esta disciplina. Este trabajo [63], titulado "sobre el electroencefalograma humano", no sólo mostraba que 
las oscilaciones regulares de corriente eléctrica pueden ser obtenidas directamente de la superficie de la cabeza en sujetos humanos, sino también que estas oscilaciones no están relacionadas ni con el flujo sanguíneo, ni con las propiedades eléctricas de la piel, aspecto clave para comenzar a relacionar la actividad muscular o cognitiva del sujeto con la señal cerebral. Posteriores estudios de Berger [64] probarían definitivamente que es el cerebro el origen de dichas ondas electroencefalográficas siendo el primero en medir e identificar la actividad oscilatoria del cerebro como las onda alpha (8-10hz). Otros científicos como Kornmüller o Fischer y Löwenbach validaron posteriormente los planteamientos de Hans Berger [65]. A partir de este momento, las señales EEG serían utilizadas para tratar de localizar patologías cerebrales y asociar señales EEG con actividad mental concreta.

En 1930, el científico inglés Grey Walter fue más allá que Berger convirtiéndose en el primer científico en detectar actividad anormal asociada a la presencia de tumores o epilepsia [66]. También fue pionero realizando adquisiciones de señal EEG con varios electrodos simultaneamente al tiempo que mostró determinados patrones cerebrales cuyos cambios estaban asociados a determinadas tareas mentales. A partir de 1940, según publicaciones ligadas al estudio de la epilepsia [67], el camino que inició Hans Berger comienza a ser recorrido por otros científicos como Adrian, Matheus y Grey Walter en Gran Bretaña; Fisher Kornmüller, Foerster y Tönis en Alemania y otros científicos como Jassper, Bishop o Lennox desde Norteamérica. Es a partir de este momento en el que las señales EEG comienzan a ser valoradas como parte del instrumental clínico, con el objetivo de realizar diagnósticos fiables sobre múltiples desórdenes neurológicos.

Partiendo de estos hallazgos, gran parte de los desarrollos en el campo del BCI han estado motivados por un intento de mejorar las vidas de sujetos que han sufrido lesiones neurológicas o neuromotoras, tales como la esclerosis lateral amiotrófica, derrames cerebrales, parálisis, tretaplegia, etc... El BCI está siendo estudiado durante los últimos 30 años y nació como una respuesta para solucionar estos graves problemas a los que se enfrentan esos pacientes que han perdido casi totalmente la movilidad de su cuerpo pero conservan intactas las funciones de razonamiento, visuales y cognitivas. Los investigadores comienzan a valerse de estas funciones para establecer mecanismos que están permitiendo a todo tipo de pacientes comunicarse 
con el exterior. En este sentido, los sistemas BCI no sólo han utilizado como fuente las señales EEG o ECoG, sino también la señal generada a partir del reconocimiento del movimiento de los ojos [68].

Las investigaciones en el campo del Brain Computer Interface son cada vez más multidisciplinares, introduciendo conceptos procedentes de la neurociencia, la psicología, las matemáticas, la ingeniería y la informática. Sin embargo, en los últimos años a medida que los sistemas BCI han ido incorporándose a experimentos que buscaban aplicaciones fuera de los laboratorios de investigación, el desarrollo en este campo ha comenzado a estar influido por la ciencia cognitiva, la ingeniería o las humanidades. El campo de la neurociencia suministra información muy valiosa sobre la estructura, funciones y mecanismos del cerebro humano. La ingeniería y los sistemas de tratamiento de señal juegan un papel fundamental en la implementación de sistemas que permitan ejecutar acciones concretas sobre el reconocimiento de los datos EEG.

Debido a que la usabilidad y fiabilidad de los sistemas BCI han sido mejoradas de una forma importante en los últimos 20 años, su aplicabilidad para todo tipo de escenarios, fuera de los usos con pacientes de movilidad reducida, ha ido creciendo de forma paralela. La introducción de sistemas BCI pasivos e híbridos han permitido que esta tecnología pueda resultar interesante también para personas sanas que realizan tareas muy específicas como usos militares, personas que interactúan con realidad aumentada, astronautas, etc. Las aplicaciones de los prototipos BCI comienzan a desarrollarse en el campo de los videojuegos y el entretenimiento, posibilitando el control de un videojuego utilizando las señales cerebrales conjuntamente con las habilidades fisicas tradicionales [69]. Los usuarios sin ningún tipo de problema motor o cognitivo, podrían beneficiarse de los avances en los sistemas BCI, utilizando esta tecnología como un input adicional al sistema de decisión.

El BCI basado en señales electroencefalográficas y entornos no invasivos no ha hecho mas que despegar, a la vista de los diversos experimentos que están mostrando el enorme potencial que encierra el análisis de la señal EEG, ya desde las primeras sesiones de test. Mejorar la precisión en la decodificación de la señal es fundamental para que los sistemas BCI avancen desde el laboratorio al mundo real, por eso algunos trabajos ya estan mostrando una alta precisión en la decodificación desde las 
primeras pruebas [70], detectando el movimiento del dedo a izquierda y derecha. El BCI basado en EEG se encuentra actualmente en un estado embrionario del que debe salir mediante la maduración de tecnologías de adquisición de señales, mejora de los algoritmos utilizados para decodificación de la señal y aumento del conocimiento sobre los mecanismos cerebrales que originan la señal en la corteza a partir de las decisiones del sujeto. En este sentido serán aspectos clave por un lado aumentar la capacidad de comunicación entre el sujeto y la máquina o interfaz, y por otro diseñar tecnología capaz de integrarse en la vida cotidiana facilitando al sujeto la ejecución de tareas rutinarias.

\subsection{Bases del modelo BCI}

El cerebro humano y una computadora clásica poseen muchas similitudes que hacen inevitable que, en ocasiones, estos sistemas sean comparados para evaluar sus rendimientos durante la ejecución de determinadas tareas. Si se tuviese que comparar el cerebro con una computadora actual, se encontrarían diferencias sustanciales tanto en su estructura y arquitectura como en las funciones desempeñadas por cada uno de los elementos. A pesar de que tanto el cerebro como la computadora han sido diseñados para solucionar problemas enormemente complejos, ambos tienen diferencias importantes que los hacen muy distintos. El modelo computacional del cerebro está basado en una arquitectura distribuída, en una red neuronal que integra tanto la memoria como la capacidad de computación. No existe una separación clara y directa entre la unidad de procesamiento y la memoria. Esta es una de las diferencias importantes desde el punto de vista estructural, ya que en una computadora tradicional existe una separación clara entre la memoria y la unidad de procesamiento. En el cuadro 2.1 se puede encontrar una comparativa sobre las diferencias estructurales y funcionales de cerebro humano y computadora. La capacidad de procesamiento ante problemas de distinta naturaleza también diferencia al cerebro de una computadora convencional. En el caso de la computadora tradicional existe una separación clara tanto entre el software (o programa) y el hardware, como entre la capacidad de procesamiento y la memoria. Por el contrario, la memoria y el aprendizaje en la red cerebral se encuentra definida y almacenada en las conexiones sinápticas, en 


\begin{tabular}{|c|c|}
\hline Cerebro & Computadora \\
\hline $\begin{array}{l}\text { Arquitectura Distribuida. No existe se- } \\
\text { paración entre unidad de proceso y me- } \\
\text { moria }\end{array}$ & $\begin{array}{l}\text { Arquitectura Harvard o Von Neuman. } \\
\text { La CPU y la Memoria están claramente } \\
\text { separadas }\end{array}$ \\
\hline $\begin{array}{l}\text { El Cerebro humano posee aproximada- } \\
\text { mente } 10^{11} \text { Neuronas con } 10^{4} \text { conexio- } \\
\text { nes cada una }\end{array}$ & $\begin{array}{l}\text { Un Chip de silicio posee } 10^{10} \text { transisto- } \\
\text { res con relativamente pocas conexiones }\end{array}$ \\
\hline $\begin{array}{l}\text { Computación paralela y conexiones } \\
\text { adaptativas de los elementos neurona- } \\
\text { les }\end{array}$ & $\begin{array}{l}\text { Computación secuencial a través de } \\
\text { CPUs y conexiones fijas }\end{array}$ \\
\hline $\begin{array}{l}\text { Excelente para resolver problemas com- } \\
\text { plejos (reconocimiento de rostros, len- } \\
\text { guaje, etc.) }\end{array}$ & $\begin{array}{l}\text { Excelente realizando cálculos ma- } \\
\text { temáticos de alta complejidad }\end{array}$ \\
\hline
\end{tabular}

Cuadro 2.1: Comparación funcional y estructural entre la computación neuronal y la de una computadora clásica.

la capacidad de producir cambios en los umbrales de excitación con el objetivo de poner en marcha un proceso de aprendizaje.

Desde el punto de vista de la rapidez, la velocidad a la que el cerebro es capaz de resolver operaciones complejas en las que intervengan raíces cuadradas, potencias o problemas geométricos será lenta en comparación con la capacidad de la computadora para resolver el problema matemático por muy complejo que este sea. Sin embargo, la computadora convencional será extremadamente lenta en resolver problemas de la vida cotidiana, analizar escenas complejas o tomar decisiones correctas del mundo cotidiano. En este último caso, los mecanismos cerebrales ganarán en velocidad al computador más avanzado.

El cerebro no constituye una máquina de propósito general que pudiera asimilarse con un computador compuesto de una unidad central de proceso y una unidad de memoria. Muy al contrario, el cerebro está compuesto por una red compleja de subsistemas altamente especializados que se encargan de realizar tareas concretas. Según se desprende de estudios recientes, a pesar de que hasta ahora la visión general sobre las funciones cerebrales ha estado exclusivamente ligada con el control motor y la coordinación, se han encontrado evidencias desde el punto de vista de la neuroanatomía, el 
comportamiento y el estudio de imagen cerebral que está cambiando profundamente esta visión. En este sentido, estudios neuroanatómicos habrían demostrado que los mecanismos cerebrales alcanzan grandes áreas de la neocorteza, incluyendo regiones prefrontales y de la corteza parietal posterior. Además, recientemente ha quedado claro que el cerebro está recíprocamente conectado con los ganglios basales y con el cerebelo, lo que podría sugerir que estas estructuras subcorticales son parte de una red densamente interconectada [71] [72]. A nivel anatómico, es sobradamente conocida la división del cerebro en dos partes bien diferenciadas: La corteza cerebral y las regiones subcorticales. Los sistemas BCI no invasivos están basados en el análisis de la señales captadas en el cuero cabelludo, debido a que cualquier tarea ejecutada por el ser humano tiene su reflejo en la corteza, concentrando en ella las principales funciones motoras, funciones de razonamiento y cognición, etc.

Años atras se ha estado trabajando en aspectos relacionadas con la naturaleza de las fuentes de la señal EEG, el origen de las mismas, asi como el análisis de sus patrones y características [73]. Algunos estudios [74] han analizado conjuntamente los resultados del análisis de la señal EEG y los obtenidos a través de la resonancia magnética funcional (fRMI). Este tipo de estudios [75] ha permitido identificar el nivel de oxígeno en sangre dependiendo de los cambios de la señal EEG asociados a cambios patológicos y psicológicos analizando ritmos cerebrales de la señal EEG.

Todos estos estudios, ayudan a conocer el comportamiento de las estructuras cerebrales relacionándolo con la señal EEG generada.

\subsubsection{Bases biológicas y modelo neuronal}

Las señales EEG constituyen la firma de los procesos que tienen lugar a nivel cerebral. En sistemas BCI basados en señales EEG, las fuentes electrofisiológicas están ligadas a mecanismos y/o procesos neurológicos que son identificados para generar las señales de control. Como ya se ha relatado, la corteza cerebral es el centro principal para el control del movimiento voluntario. Utiliza la información que recibe del cerebelo, de los ganglios basales y de otros centros del sistema nervioso central, así como las señales de retroalimentación procedentes de la periferia, con el objetivo de controlar el movimiento voluntario. Además, la corteza cerebral proporciona las funciones intelectuales avanzadas del ser humano, como capacidades de memoria y 
de recuerdo, así como otras funciones cognitivas superiores. Hace varias décadas, la asociación entre los procesos cognitivos y un patrón de señal EEG era objeto de estudio en la comunidad científica [76].

Se argumentaba que desde el análisis realizado por Hans Berger sobre los cambios de la señal cerebral relativa a la actividad mental, diversos estudios han intentado relacionar ciertas señales EEG espontaneas con funciones mentales complejas en adultos. En aquellos años, dichos esfuerzos no consiguieron desarrollar un modelo que permita relacionar los patrones EEG a determinados aspectos cognitivos. Estudios recientes [77] han puesto de manifiesto que la oscilación $\theta(3.5-7.5 \mathrm{~Hz})$ podria jugar un rol de integración importante en la organización de la actividad cerebral. En toda actividad relacionada con los mecanismos de la memoria se ven involucrados una gran variedad de procesos cognitivos. Por ello, estos autores sugieren que la coordinación se realiza mediante un mecanismo de integración cerebral con presencia de actividad $\theta$. A nivel de proceso, se analizá la sincronización interregional de la banda theta para integrar las estructuras cerebrales necesarias para el funcionamiento de la memoria.

\subsubsection{La corteza cerebral y áreas de Brodmann}

La corteza cerebral es la encargada de percibir, comprender e integrar los diferentes estímulos y en ella podemos analizar toda la actividad cerebral del movimiento voluntario y la mayor parte de procesos cognitivos de una forma fiable. Desde el punto de vista anatómico y superficial, la corteza cerebral se divide en lóbulos, giros y surcos. Los giros son elevaciones repentinas de la superficie cerebral que aparecen a partir de pliegues de la corteza sobre sí misma. Los giros o circunvoluciones están separados entre sí por surcos. A los surcos que poseen una profundidad considerable se les denomina cisuras, que separan zonas importantes de la corteza cerebral tanto desde el punto de vista anatómico como funcional. Es el caso de la cisura interhemisférica, que se encuentra en la línea de separación de los dos hemisferios. También cabe mencionar o las cisuras de Silvio o Rolando que separan el lóbulo frontal del temporal y el lóbulo parietal del frontal respectivamente. La división originada por estas cisuras dividen los hemisferios cerebrales en los lóbulos frontal, occipital, parietal y temporal, que se localizan bajo la masa osea craneal del mismo nombre. 


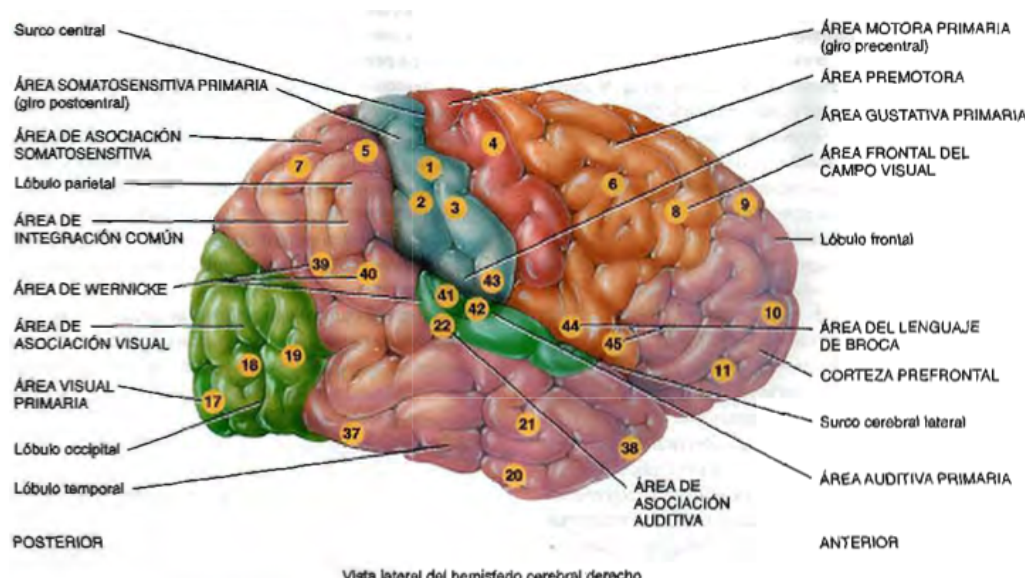

Figura 2.3: Estructura de la corteza cerebral. Hemisferio derecho [78]

Analizando la estructura de la corteza cerebral, se observa una arquitectura caracterizada por seis capas que componen su estructura en la mayor parte de las zonas corticales, formando la neocorteza o isocorteza. La corteza cerebral de mamíferos está representada por una lámina de sustancia gris que cubre los hemisferios cerebrales. Es una estructura extremadamente compleja en la que los distintos órganos sensoriales aparecen representados en determinadas áreas o zonas conocidas como áreas sensoriales primarias. Desde el sentido del olfato hasta el de la visión, cada modalidad sensorial posee su propio territorio cortical, el cual mantiene una localización relativamente constante en todos los mamíferos. Además de estas áreas sensoriales primarias, la corteza cerebral posee múltiples áreas somáticas secundarias, de asociación y motoras, en las que los diferentes sistemas aferentes corticales y de asociación son finalmente elaborados en complejas respuestas de aprendizaje, memoria y comportamiento [79]. Desde el punto de vista anatómico, la información recibida por la corteza cerebral llega a traves del tálamo. Esta estructura está formada por una acumulación de grupos neuronales en el diencéfalo y toma parte en el desarrollo de funciones sensitivas, motoras y límbicas. El tálamo se divide en núcleos que, en función de su especialización, mandan información a cada una de las partes de la corteza cerebral encargada de la función asociada a dicho núcleo. Las neuronas de los ganglios basales juegan un papel importante en los instantes previos al inicio del movimiento. Este hecho, junto con el análisis derivado de los efectos que produce en el movimiento voluntario la alteración de los ganglios basales [80] han reforza- 
do la idea de que los ganglios basales intervienen claramente en la planificación del movimiento y la acción voluntaria. La Figura 2.4 describe las principales conexiones excitadoras (línea punteada) e inhibidoras entre la corteza, el tálamo y los ganglios basales.

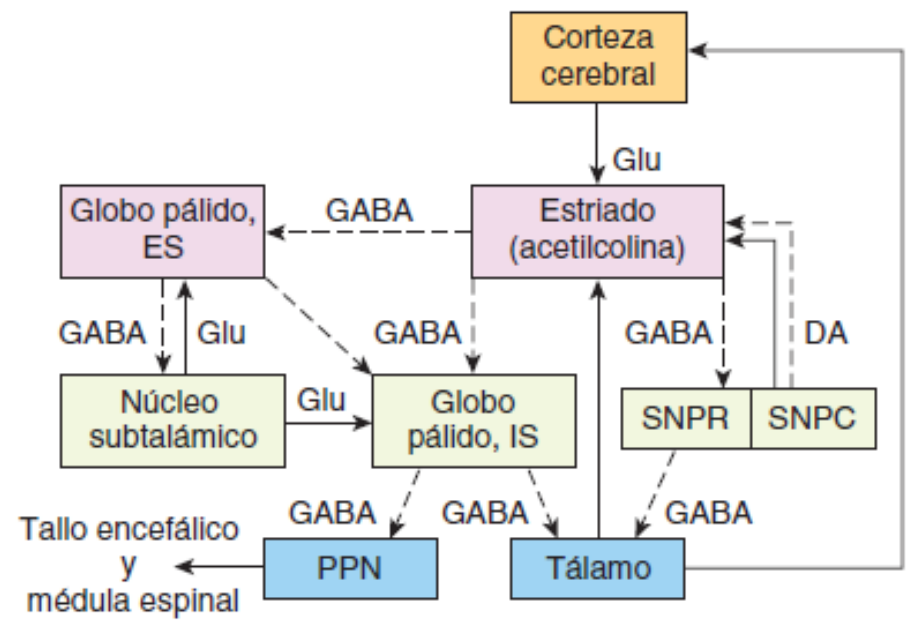

Figura 2.4: Conexiones principales entre corteza, tálamo y ganglios basales [81]

Las células más abundantes y de mayor presencia en la corteza cerebral son las células piramidales, origen de la vía corticoespinal, y que representan la principal vía de eferencia, permitiendo la transmisión de información desde la corteza. Cabe destacar que el espesor de las seis capas de la neocorteza no permanece constante en toda su estructura, dependiendo el grosor de la función desarrollada en cada caso. Las diferentes vías tanto sensoriales como motoras envían impulsos que discurren a través de los diferentes tractos neuronales hacia la corteza. En este sentido, las oscilaciones generadas en el interior de las capas de la corteza cerebral así como entre otras estructuras del sistema nervioso como los ganglios basales, han sido estudiadas para profundizar en su relación con las oscilaciones electroencefalograficas [82] [83]. El conjunto de fenómenos que dan lugar a la generación de la señal electroencefalográfica no tiene su origen en otras estructuras distintas del cerebro, sino que proceden directamente de la corteza cerebral. Además, a través del análisis de la señal EEG es posible analizar otras estructuras subcorticales debido a que la señal EEG contiene información integrada sobre la interacción entre estructuras corticales 
y subcorticales que se proyectan en la superficie de la corteza para ser captadas por sistemas BCI [82] [84]. Contrariamente a lo que podría pensarse, las capas de la corteza cerebral no interaccionan entre si a través de un patrón horizontal, sino que las neuronas de las distintas capas operan de forma mayoritaria con aquellas células que se encuentran directamente en la capa superior o inferior de una célula concreta. Hace algunas décadas, se pudo identificar y corroborar esta organización en forma de columnas [85]. Recientemente se ha confirmado esta teoría, profundizado en el estudio para definir anatómicamente las columnas corticales a través del análisis de la excitación sináptica de las neuronas contenidas en una columna concreta, como las pertenecientes al cortex somatosensorial primario de un ratón [86]. Por tanto, la semejanza funcional de estas células a lo largo de las seis capas sugiere que la unidad más sencilla de la corteza es una columna de céulas orientadas verticalmente con similares funciones. Este hecho refuerza la posibilidad de extraer información útil como entrada a un sistema BCI, procedente de las distintas zonas del cortex cerebral, a traves de la señal EEG. El origen de todo se encuentra en los procesos sinápticos producidos en las distintas capas de la corteza, así como en las distintas estructuras cerebrales, que generan un reflejo en la señal EEG que puede ser captada mediante electrodos no invasivos directamente desde el cuero cabelludo. Hace algunas décadas, algunos autores [82] demostraron que las lesiones producidas en determinadas estructuras subcorticales, modifican de forma significativa la señal EEG. Por tanto, es posible apoyarse en este hecho para detectar cambios funcionales o anatómicos en las estructuras subcorticales a través del análisis de la señal EEG generada.

Debido a esta especialización en forma de columnas de las distintas neuronas de la corteza cerebral, se ha tratado de asociar áreas concretas de la corteza cerebral con partes del cuerpo o funciones sensoriales. En este sentido, la estimulación de ciertas regiones motoras y somatosensoriales, llevaron a Penfield y su equipo a la identificación de algunas regiones que se encontraban asociadas a partes concretas del cuerpo como la mano, pierna o cara [87]. Desde entonces, han sido muchos los autores que han ampliado estas conclusiones y utilizado el trabajo de Penfield para formular nuevos planteamientos a partir de la representación visual de las diversas funciones del cuerpo innervadas en la corteza cerebral, conocida como el Homúnculo de Penfield [88]. El homúnculo cerebral es el resultado visual de las investigaciones llevadas a 
cabo sobre aquellas partes del cerebro encargadas de determinadas funciones motoras y sensoriales. Representa la innervación en la corteza cerebral de ciertas partes del cuerpo, identificando las áreas responsables de las sensaciones táctiles, la presión, la temperatura, movimientos de zonas concretas, etc. En el homúnculo cerebral se muestran claramente partes del cuerpo que tienen un mayor tamaño respecto de la proporcionalidad lógica de un cuerpo humano normal, esto se debe a que en este caso, cada órgano se encuentra descrito por la sensibilidad del área cortical y no por el tamaño de la misma.

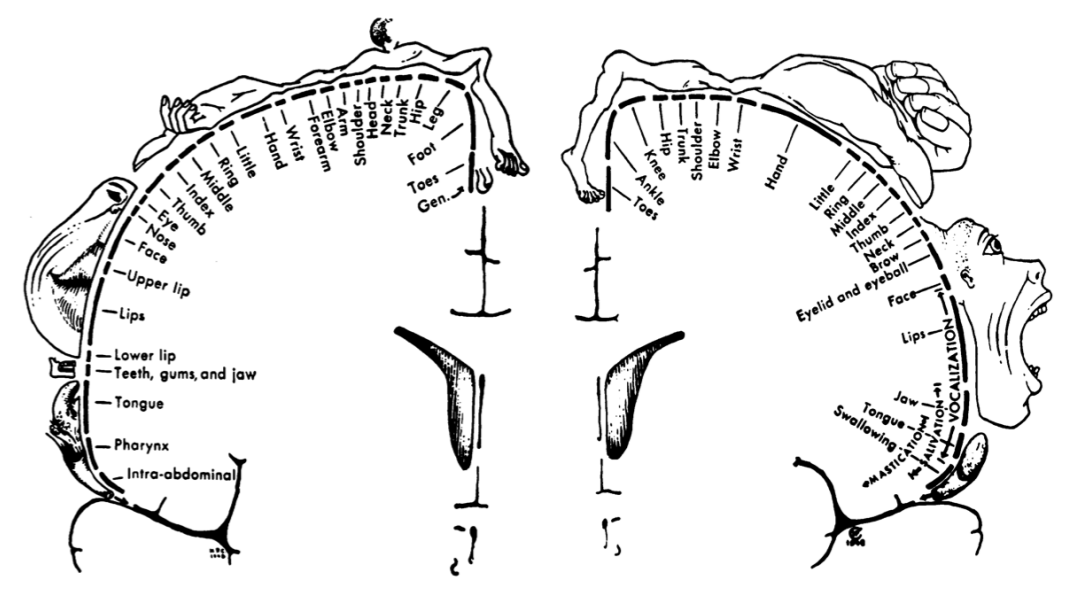

Figura 2.5: Primer mapa dell homúnculo motor y sensorial [88]

El homúnculo motor es diferente entre sujetos en función de la edad. Esto es debido a que la representación de una extremidad en un sujeto de corta edad difiere en gran medida respecto de un sujeto adulto. Además, estas diferencias también se encuentran entre sujetos de la misma edad, ya que pueden existir diferencias significativas en lo que a capacidad de movimientos se refiere, debido a las diferencias organizativas respecto de las funciones de las áreas cerebrales relacionadas. Estas diferencias son consecuencia de la presencia en algunos casos de un mayor o menor número de neuronas asociadas a dichas áreas. Sin embargo, está fuera de toda duda que la corteza cerebral posee áreas bien diferenciadas a nivel superficial donde se localizan distintas funciones motoras, sensoriales y cognitivas cuya información puede ser extraida en forma de impulsos eléctricos [89] [90].

A partir de todo lo desarrollado anteriormente, el investigador Korbinian Brod- 
mann (1968 - 1918) trató de sectorizar la corteza en distintas zonas basándose en las diferencias estructurales y funcionales de cada sector. Estas zonas son conocidas como "áreas de brodmann" y han sido numeradas desde la 1 hasta la 52 [91]. Esta numeración se realizó teniendo en cuenta desde las diferencias en el espesor de las distintas capas que forman la corteza cerebral, hasta la forma y tamaño de las neuronas que componen una zona concreta de la corteza cerebral. La numeración de cada una de estas zonas está orientada exclusivamente a la identificación de las mismas, sin que el orden guarde ningún tipo de relación con la función desempeñada por cada una de las áreas. De hecho, no existe ninguna relación en el orden de los números debido a que Brodmann delimitó y numeró cada una de las áreas en orden aleatorio a partir de su identificación y asociación con funciones concretas. Desde que estas áreas fueron descritas, la comunidad científica ha demostrado en diversos estudios la validez de los postulados enunciados por Brodmann.

La corteza somatosensitiva primaria comprende las áreas de brodmann 1, 2 y 3, y realiza funciones relativas a la recepción de sensaciones procedentes del tacto, articulaciones, músculos y otras estructuras situadas en la parte contralateral. Existen estudios que han corroborado que la mano está totalmente representada en estas áreas [92]. El área motora primaria ocupa el área precentral y corresponde al área 4 de brodmann. Debido a que se corresponde con el homúnculo motor, anteriormente descrito, el tamaño de cada una de las partes asociadas a acciones concretas será directamente proporcional al nivel de complejidad de dichas acciones. El área 5 y 7 forma el área sensitiva secundaria ubicada en el lóbulo parietal superior. La función fundamental de estas áreas está relacionada con los movimientos voluntarios; su lesión incapacita al sujeto para ejecutar movimientos concretos, especialmente cuando se tiene una referencia externa en el espacio. La respuesta a nivel neuronal dentro del área de Brodmann 5 ha sido estudiada arrojando como resultado una sensibilidad a la estimulación mecánica suave del tejido de la piel así como a la rotación articular [93]. El área 6 forma el área motora suplementaria. Situada junto al área motora primaria, es la encargada del control de los movimientos secundarios como consecuencia de un movimiento voluntario. Además de las funciones motoras, se han estudiado las funciones mentales no motoras relacionadas con tareas verbales, espaciales y numéricas [94]. Los resultados obtenidos sugieren que esta zona es importante en las tareas 
de asociación y en los comportamientos tanto motores como no motores. Tanto las áreas motoras como las premotoras pueden englobarse, desde un punto de vista funcional, como una parte del sistema para el control del movimiento voluntario. Las áreas 8, 9, 10, 11 y 12 forman el área prefontal, ejecutando procesos relacionados generalmente con el pensamiento, el juicio, la voluntad o el razonamiento. En el caso del área prefrontal, se ha demostrado la utilidad de los circuitos frontales subcorticales para explicar gran parte del comportamiento humano [95]. Las fibras nerviosas que parten de la corteza motora hacia los núcleos de los nervios craneales forman el tracto corticubulbar, de igual forma que las fibras nerviosas que atraviesan por la línea media las pirámides medulares forman el tracto corticoespinal. Las áreas corticales de las que se originan ambos sistemas son aquellas en las que una estimulación produce un movimiento rápido y opuesto. La estructura principal es la corteza motora primaria, sin embargo también ha de citarse al área motora suplementaria y la corteza premotora, asi como el área sensitiva-somática, cuya estimulación puede generar respuestas motoras [96].

El área 17 constituye la zona visual primaria, encargada de integrar la información espacial con la información visual procedente de los ojos. La imaginería visual está siendo utilizada en una gran variedad de actividades mentales e incluso como base para interactuar con un sistema BCI. La contribución de la corteza visual primaria, concretamente del área 17 de Brodmann, a la imaginería visual mental ha sido examinada por algunos autores mostrando activación del área 17 cuando el sujeto ejecuta tareas de visualización mental [97]. Es en el área psicovisual, compuesta por las áreas de Brodmann 18 y 19, donde tiene lugar la recepción de los estímulos visuales complejos. Experimentos en monos han demostrado que producir daño cerebral sobre estas zonas generan un deterioro notable en la capacidad visual [98]. Desde el punto de vista del procesamiento de los estímulos auditivos, existe una zona concreta donde este proceso tiene lugar, las áreas 41 y 42 (corteza primaria) y el área 22 (corteza secundaria). Esta última zona, el área 22, es normalmente considerada como la contenedora del área de Wernicke, perteneciente al cortex asociativo realizando funciones específicamente auditivas y asociada de forma muy concreta a funciones lingüísticas. La producción del lenguaje está muy ligada al área de Wernicke y su análisis puede proveer información sobre la producción del habla que sigue 
a los movimientos de la cara. A partir de estas predicciones, se podría establecer un mecanismo mediante el cual los movimientos de las articulaciones están, de forma precisa, ligados a la intención del habla [99]. Además, los resultados relacionados con el aprendizaje de una segunda lengua mostraron que el comportamiento del área de Wernicke no sufría variación dependiendo de la edad en la que se adquiría la segunda lengua [100], al contrario de lo que ocurre con el área de Brocca. También juega un papel importante en el campo del BCI el área de Wernicke. El análisis de este área es fundamental para los sistemas de reconocimiento del habla imaginada o "silent speech interfaces". Algunos autores ya han demostrado en estudios recientes el potencial de estos sistemas BCI [101].

Las áreas 23, 24, 28, 29, 30 y 35 corresponden al área límbica, cuyas funciones principales están asociadas al campo de las emociones, así como las áreas 28 y 34 están asociadas fundamentalmente al sentido del olfato. Por otro lado, forman parte del área de Wernicke las áreas 39 y 40 de Brodmann, encontrándose ubicado el cortex gustativo en el área 43. La función de producción del habla y comprensión del lenguaje hablado y escrito, así como el procesamiento del lenguaje, se encuentran en las áreas 44 y 45, en el área de Broca. Un haz nervioso conecta el área de Broca con el área de Wernicke donde también se desarrollan funciones de procesamiento lingüístico. El área de Broca está encargada de la producción del habla, el área de wernicke de la percepción del habla, y juntas son las responsables del lenguaje. El análisis de la coherencia entre las señales precdedentes de electrodos colocados en el área de Broca y Wernicke, y entre las áreas temporales derecha e izquierda, han permitido reforzar teorias sobre la esquizofrenia en pacientes que sufren alucinación auditiva esquizofrenica [102]. El papel del área de Broca en la producción del habla se pone de manifiesto en recientes estudios [103], a partir de los cuales se puede afirmar que la estimulación transcraneal en el area de Broca, puede ser utilizada como instrumento terapéutico en pacientes con trastornos del lenguaje debidos a daños cerebrales.

El mapa cortical funcional es, en realidad, un poco más complejo que el desarrollado por Brodmann. Sin embargo, la comunidad científica ha venido reconociendo en multitud de estudios la utilidad de trabajar con el modelo de Brodmann a la hora de realizar análisis sobre la organización funcional de la corteza cerebral. Prueba de ello 
son los recientes estudios sobre áreas de Brodmann que podrían tener asociadas otro tipo de funciones adicionales a las conocidas hasta ahora. Es el caso de un estudio [104] en el que se especula con las funciones del área 47, tradicionalmente asociada al procesamiento lingüístico, y que a tenor de los resultados experimentales obtenidos tras examinar la respuesta cerebral a un experimento auditivo de trece sujetos, podría tener funciones relacionadas con el procesamiento detallado y la integración estructurada de estímulos concretos. Existen también estudios que han profundizado en definir de forma más exacta la frontera de determinadas áreas de brodmann, este es el caso de un trabajo relativamente reciente [105] sobre la definición experimental de las áreas 17 y 18 a través del análisis de 10 estructuras cerebrales de diferentes sujetos. A partir de aquí, algunos estudios han profundizado hasta el punto de desarrollar herramientas específicas [106] para conseguir combinar información estructural procedente de los mapas cerebrales existentes con la información funcional obtenida a partir de técnicas avanzadas de neuroimagen. Otros estudios se han centrado en demostrar que determinadas acciones activarían partes específicas de la corteza premotora, así como en determinar hasta qué punto la presencia de un objeto influye en el análisis de una acción observada [107]. Cuando se actúa sobre un objeto, tiene lugar un análisis específico de dicho objeto en el lóbulo parietal, distinto del procesamiento semántico que tiene lugar en el lóbulo temporal. Según dicho estudio, cabe preguntarse si la observación de acciones relacionadas con objetos concretos generan dicho análisis. Los resultados de dicho estudio son concluyentes, ya que cuando un sujeto observa acciones realizadas por otro sujeto se produce la activación de diferentes sectores de la corteza premotora. A modo de ejemplo, la realización de acciones relacionadas con la boca produce una activación bilateral de la zona ventral del área 6 y 44 además de la activación derecha del área 45. Además, la observación de acciones relacionadas con los pies, producen una activación del sector dorsal del área 6 de forma bilateral. La importancia de estas acciones reside en el establecimiento de la diferencia entre aquellos experimentos en los que tienen lugar acciones relacionadas o no con la presencia de objetos. En el momento en el que un objeto sea el objetivo de una acción, el lóbulo parietal se activará fuertemente.

Las oscilaciones generadas por las señales electroencefalograficas reflejan procesos cognitivos y representaciones sensoriales. Sin embargo, mientras que la relación entre 
la amplitud y las variables cognitivas-sensoriales ha sido ampliamente estudiada, recientes estudios revelan que los trazados de oscilaciones dinámicas (patrones de fase) pueden transportar información sobre dichos procesos en mayor grado que en el caso que la amplitud de la señal. Para extraer las correlaciones entre los patrones oscilatorios de fase, algunos autores [108] han comparado la selección de estímulos de los umbrales de respuestas neuronales y las oscilaciones electroencefalograficas a partir de estímulos auditivos. Este estudio concluyó que los estímulos que pueden ser discriminados por patrones neuronales relacionados con la respuesta neuronal pueden ser también discriminados por patrones de fase, no ocurriendo lo mismo en el caso de utilizar como parametro la amplitud de la señal. Esto revela un nivel importante de interrelación entre las señales electroencefalograficas y la respuesta neuronal, más allá de las posibles relaciones en las amplitudes simples de ambas señales.

\subsubsection{Relación estímulo-respuesta}

La actividad neuronal es generada a partir del procesamiento que el cerebro hace de determinados estimulos externos. Una vez analizado de forma visual el resultado de la monitorización cerebral y observando las señales generadas por cada neurona, cabe preguntarse:

1. ¿Cómo podrían usarse estas respuestas para extraer información útil y determinar el proceso cerebral o estímulo al que ha sido sometido el sujeto?

2. ¿Son estas respuestas independientes entre cada neurona o existe algún tipo de respuesta conjunta generada por grupos de neuronas similares o cercanas?

El objetivo principal de todo sistema BCI es conseguir la decodificación total o parcial de la señal cerebral. Si fuese posible conocer la respuesta generada ante un estímulo desconocido, seria posible obtener dicho estímulo aplicando métodos de procesado y clasificación de señal a la respuesta cerebral que originó dicho estímulo. Dicha respuesta, no es otra que la señal EEG generada durante la presentación de un estimulo o la ejecución de una acción. La señal EEG puede ser decodificada cuando se pretende obtener el estímulo que generó una respuesta conocida. Sin embargo, también puede recorrerse el camino inverso, y será posible hablar de codificación de 
la señal cerebral, cuando lo que se busca es estimar la respuesta en términos de señal EEG, conociendo previamente el estímulo que se aplica durante el proceso. En el caso de la codificación de la señal, se busca la forma en la que las estructuras cerebrales codifican ese estímulo en forma de respuesta cerebral [109]. La codificación de la señal cerebral y su representación probabilística está basada en la probabilidad de obtener una determinada respuesta dado un estimulo concreto $\mathrm{P}$ (Respuesta-Estímulo). Por el contrario, la probabilidad de obtener un estimulo dada una respuesta conocida es la base de la decodificación. Hasta ahora, se ha asumido que la respuesta cerebral se corresponde con el trazado electroencefalográfico generado. Por tanto, podemos definir la respuesta como la probabilidad de obtener un potencial de acción determinado en una neurona o conjunto de neuronas.

Los sistemas BCI basan su efectividad en el estudio de la relación entre el estimulo y la respuesta a traves de algoritmos de procesado de señal cerebral y el conocimiento de las teorias biológicas que dan lugar a los procesos neuronales.

\subsection{Generación de la señal EEG}

La señal EEG es captada tras la excitación de las células nerviosas que reciben estímulos eléctroquímicos, cuyo umbral de excitación es relativamente bajo. La información es transportada de una estructura celular a otra tras la generación y transmisión de los impulsos nerviosos. Estos impulsos nerviosos transmitidos por los nervios son conocidos como potenciales de acción. El proceso mediante el cual se genera un potencial de acción en una célula está basado en un intercambio de iones a través de la membrana neuronal, en este sentido un potencial de acción se genera gracias a un cambio temporal en el potencial de la membrana neuronal. Se pueden iniciar a través de los nervios sensoriales como respuesta a estímulos químicos, de presión, luz, etc. El potencial de acción es transmitido a través de los axones hasta la terminación de dichas estructuras. Las unidades de información del sistema nervioso son las excitaciones generadas por los potenciales de acción. La primera manifestación de la aproximación del potencial de acción comienza con la despolarización de la membrana, tal y como puede apreciarse en la Figura 2.6. Una vez superado el umbral de activación, la velocidad de la despolarización se incrementa hasta que se 
alcanza el valor de $+35 \mathrm{mV}$, momento en el cual el potencial cae de nuevo hasta el potencial de reposo [96]. Esta subida y bajada repentina en el potencial, generándose un impulso conducido al estimular el axón, constituye el potencial de acción. Para que un potencial de acción sea generado y viaje a través de la estructura nerviosa, es necesario que el estímulo que lo genera esté por encima de un determinado umbral. En reposo, la membrana celular se encuentra polarizada con cargas positivas alineadas en el exterior y cargas negativas en su interior. El proceso comienza cuando las dendritas de una célula nerviosa abren los canales de sodio debido a la recepción de un estímulo. Las cargas positivas son extraidas y la polaridad de la membrana disminuye, generando una respuesta local hasta alcanzar o no un nivel determinado. En este punto es en el que se determina si el estímulo es lo suficientemente importante como para generar un potencial de acción. Cabe destacar que aquellos estímulos que no consiguen superar el umbral de activación, no producen un potencial de acción aunque sí poseen un efecto sobre el potencial de membrana. El proceso continuará si el estímulo es capaz de llevar el potencial interior hasta superar los $-55 \mathrm{mV}$. Antes de alcanzar el valor definitivo de reposo, alrededor de $-70 \mathrm{mV}$, se produce la hiperpolarización, un fenómeno que lleva al potencial de acción a valores cercanos a los $-90 \mathrm{mV}$ antes de estabilizarse. La importancia de la hiperpolarización como paso previo a la entrada en el potencial de reposo se justifica debido a que gracias a este periodo se asegura que el potencial de acción enviado a través del axón discurre en una dirección, evitando cualquier estímulo que pudiera haber sido ya enviado para ejecutar alguna ación. Al final del proceso, la estructura neuronal se encuentra lista para recibir un nuevo estímulo [81]. 


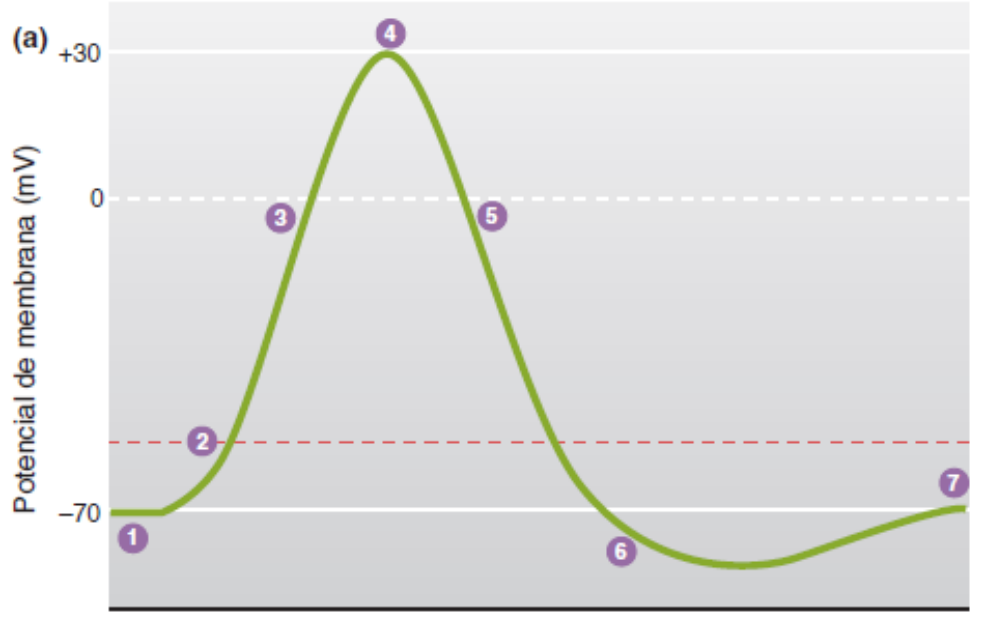

Figura 2.6: Registro del potencial de acción en una neurona [81]

En 1952, los cientificos Alan Lloyd Hodgkin y Andrew Huxley propusieron un modelo que describe la forma en la que se inician y propagan los potenciales de acción a lo largo del circuito neuronal [110]. Utilizando para este análisis un axón gigante de calamar, fueron capaces de desarrollar un modelo que explicaba los mecanismos moleculares que dan lugar a la iniciación y propagación de los potenciales de acción. El trabajo de Hodgkin y Huxley describe el proceso de transmisión a lo largo del axon. Su aportación se puede dividir en dos partes. Una es el proponer un mecanismo mediante el cual el voltaje mantiene su forma al propagarse: el flujo de iones de sodio y de potasio a través de la membrana del nervio. La otra es el planteamiento de un modelo matemático que permite cuantificar y verificar la propuesta. La validez del modelo ha quedado establecida al hacer predicciones que coinciden con las observaciones experimentales, la de mayor éxito es la predicción de la velocidad del impulso: el modelo matemático predice una velocidad de $18.8 \mathrm{~m} / \mathrm{seg}$, mientras que los experimentos con el axon gigante del calamar dieron una velocidad de 21.2 $\mathrm{m} / \mathrm{seg}[111]$.

El estudio sobre la generación de estas señales cerebrales, procedentes de los impulsos eléctricos durante la ejecución de una tarea concreta motora o cognitiva, puede aportar gran cantidad de información sobre el funcionamiento de los procesos cerebrales. Sin embargo, es evidente que modelar la actividad neuronal entraña una dificultad mucho mayor que modelar la actividad de cualquier otro órgano o parte del 
cuerpo humano debido la alta complejidad de su morfología y su multifuncionalidad. Además, se añade una dificultad extra cuando se intenta modelar dicha actividad y extraer información útil mediante el análisis de señales EEG captadas en la superficie del cuero cabelludo. La señal captada no procede únicamente de la zona de la corteza cerebral donde se encuentra situado un electrodo. Esta señal es la suma de señales EEG procedentes de fuentes de señal alrededor de la zona donde el electrodo capta el potencial eléctrico (Figura 2.7).

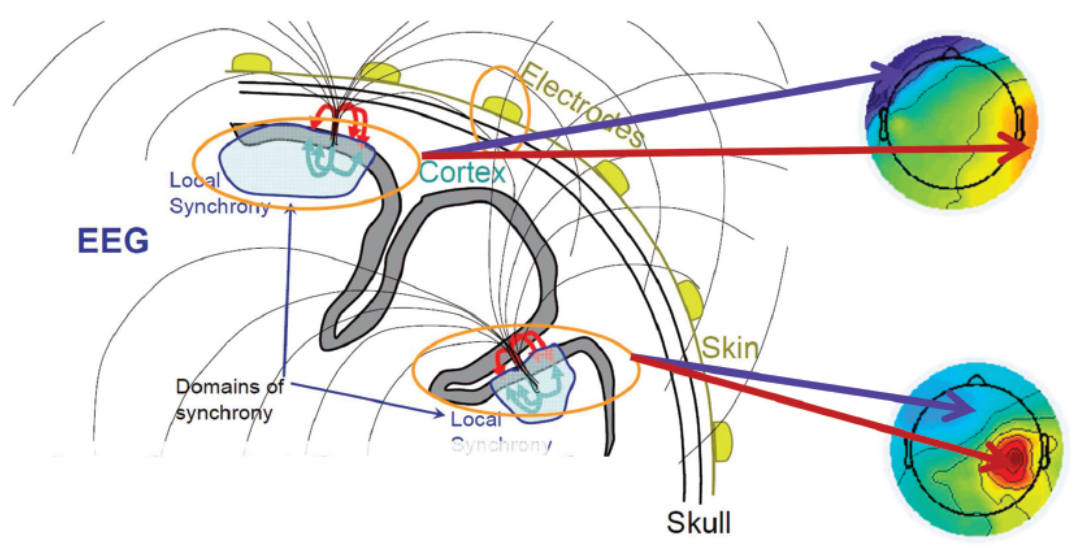

Figura 2.7: Proyecciones desde dos fuentes de señal cercanas entre sí hasta la zona donde los electrodos captan la señal EEG. El color azul indica campo eléctrico negativo, el campo rojo indica campo eléctrico positivo [28]

Existen diferentes estudios relacionados con la localización de las fuentes de la señal cerebral [112] [113] a partir del análisis de las señales EEG. Recientemente, algunos autores demostraron la utilidad que tiene el análisis de las fuentes EEG cuando se trata de estudiar las propiedades cerebrales y neuronales en la neurociencia clínica y cognitiva [114]. La mayor parte de las aplicaciones del EEG fracasan a la hora de capitalizar la información disponible en los datos registrados, como es el caso de la localización de las fuentes activas en el cerebro. La localización de las fuentes de señal a nivel cerebral a partir de una medida realizada en la corteza cerebral es posible a partir de la resolución del problema inverso. Introduciendo determinados condicionantes a priori, el problema inverso puede ser resuelto permitiendo la localización de 
la mayor parte de las fuentes de generación de señal cerebral de forma precisa. Como conclusión, los autores pusieron de manifiesto que las señales electroencefalograficas constituyen una herramienta importante para el estudio de los circuitos neuronales.

\subsubsection{Ritmos cerebrales}

Es bien conocida la existencia de cinco tipos de señales cerebrales caracterizadas por sus rangos de frecuencia. Este tipo de señales, desde la frecuencia más baja hasta la más alta son conocidas como: alfa $(\alpha)$, theta $(\theta)$, beta $(\beta)$, delta $(\delta)$ y gamma $(\gamma)$.

Los ritmos cerebrales son patrones de la señal EEG captada identificados por su amplitud o frecuencia caracteristicas. Los ritmos cerebrales no son más que fluctuaciones de la señal cerebral a frecuencias concretas que están asociados a determinados estados cerebrales tales como el descanso, sueño profundo, vigilia, etc. Estos ritmos cerebrales han sido estudiados ampliamente en el campo de las neurociencias y en diversos estudios se ha analizado la correspondiencia entre estos ritmos y los estados cerebrales que representan [115] [116] [117].

En un estado de reposo y ojos cerrados, la actividad electroencefalografica genera ondas regulares con una frecuencia de entre 8 y $13 \mathrm{~Hz}$ con una amplitud de entre 50 y $100 \mu \mathrm{V}$ conocidas como ritmos $\alpha$. El origen fisiológico de las ondas alfa es todavía desconocido y existen diversos estudios que tratan de arrojar luz sobre los procesos asociados a esta banda de frecuencias. Algunos autores incluso han comprobado la existencia de ritmos alpha de similar frecuencia y ancho de banda captados en animales desde el cortex visual para estudiar los orígenes neurofisiológicos de la actividad alpha [118]. En el caso de los ritmos $\alpha$, recientes estudios han demostrado que las alteraciones en estos ritmos producen cambios en la capacidad de atención y concentración durante la realización de tareas de aprendizaje [116]. A pesar de que el rol de las oscilaciones alpha en tareas de atención espacial, memoria y percepción está ampliamente documentada, algunos autores [115] sostienen que el papel detallado de los ritmos alpha en el aprendizaje percibido y su contribución a la variabilidad observada en los resultados del aprendizaje precisan de una mayor evaluación empírica y teórica. La combinación de diferentes medidas incluyendo el comportamiento, la actividad cerebral con una alta resolución temporal y espacial, así como el modelado computacional será crucial para superar las dificultades para comprender el enlace 
directo entre las oscilaciones de dichas ondas alfa y la percepción del aprendizaje. Este sera el primer paso para utilizar dichos hallazgos tanto en el campo clínico como en la vida cotidiana.

Cuando el sujeto se encuentra despierto y concentrado, la actividad electroencefalográfica presenta una frecuencia de entre 14 y $30 \mathrm{~Hz}$ y un voltaje inferior al registrado para el caso de los ritmos alfa. Este patron es conocido como ritmo $\beta$. El ritmo beta está generalmente asociado al pensamiento activo, procesos de atención, resolución de problemas concretos, etc. Este ritmo se encuentra en adultos sanos y, según algunos autores, un alto nivel de ritmo beta está originado por estados de pánico [119]. La alteración de los patrones EEG $\alpha$ y $\beta$ ha sido analizada en el pasado para demostrar la influencia que determinados compuestos como las benzodiacepinas ejercen de forma clara en la atención de un sujeto. Analizando los ritmos EEG ha podido encontrarse un deterioro en la capacidad atencional y de concentración como consecuencia de la ingesta aguda o cronica de benzodiacepinas. Estos efectos residuales en la atención se han encontrado también en los cambios que estos medicamentos provocan en la actividad electroencefálica espontánea y en la actividad cerebral como respuesta a estímulos externos [120]. Todavia hoy, algunos estudios afirman que el papel de las oscilaciones generadas en la banda $\beta$ no está suficientemente aclarado [121]. De este estudio, podria desprenderse que la actividad en la banda beta está relacionada con el mantenimiento del estado cognitivo en el instante actual. Dichos autores han estudiado la posibilidad de que las oscilaciones beta se manifiesten con mayor fuerza en el caso de que el mantenimiento del "status quo. ${ }^{\text {es }}$ algo esperado, que en el caso de que se espere un cambio. Ademas, se sugiere que un incremento en la actividad beta puede causar una persistencia anormal del "status quo", generando un deterioro en el control cognitivo y el comportamiento flexible.

La señal electroencefalográfica presenta frecuencias de entre 30 a $80 \mathrm{~Hz}$ correspondientes al ritmo $\gamma$ cuando el individuo concentra toda su atención en algo y se encuentra en estado de alerta. Recientemente se ha analizado la actividad Gamma tras realizar un experimento con sujetos a los que se les presentaban estimulos relacionados con un objeto concreto, para que fuese percibido por sentidos distintos [122]. En concreto se presentaban estimulos visuales y estímulos auditivos, con muy poco tiempo de diferencia entre ellos. Una parte de estos estimulos era semánticamen- 
te incongruente, mientras se mantenía el resto en perfecta congruencia semántica. Se argumenta que la actividad oscilatoria de la banda gamma juega un papel importante para la integración mental de las caracteristicas de determinados objetos. Tambien se sugiere que estas oscilaciones están ligadas al establecimiento de relaciones entre información especifica extraida de los objetos y la información relacionada en la memoria. Los datos obtenidos, han soportado la hipótesis de que la actividad oscilatoria en la banda gamma esta relacionada con el procesamiento multisensorial. La sincronización de la banda gamma entre la corteza auditiva derecha e izquierda en pacientes con esquizofrenia ha sido utilizada por algunos autores para estudiar las alucionaciones auditivas [123]. Los resultados sugieren que la sincronía de largo alcance en las oscilaciones gamma se encuentra alterado en pacientes con esquizofrenia. Este déficit está relacionado directamente con los síntomas clínicos de la enfermedad como las alucionaciones auditivas. Esto puede deberse a la existencia de un problema entre las conexiones de la corteza, el tálamo y la formación reticular (ver Figura 2.4), que genera una alteración en la sincronización de las ondas gamma.

Durante las distintas fases del sueño aparecen los ritmos $\theta$ y $\delta$. La Figura 2.8 muestra el ritmo $\theta$ que aparece en la fase 1 del sueño de un sujeto con una frecuencia de entre 4 y $7 \mathrm{~Hz}$. En una etapa más avanzada del sueño, en la tercera fase, aparece el ritmo $\delta$. La banda delta suele encontrarse en el rango $0.5-4 \mathrm{~Hz}$ y está fundamentalmente asociada con el sueño profundo, pudiendo estar presente también en otros estados. Aplicando métodos de análisis de señal es posible diferenciar la señal que contiene un ritmo Delta, de señales muy similares pero de naturaleza bien distinta como las asociadas a movimientos musculares originados en el cuello o la mandíbula. Cada vez más investigaciones sugieren que las oscilaciones lentas en la corteza auditiva, por debajo de $10 \mathrm{~Hz}$, aparecen para seguir el habla escuchada y otras señales auditivas complejas [124]. La conclusión mas importante de un reciente estudio [3] se basa en que las señales EEG en el rango de frecuencias tetha y alpha están relacionadas con la cognición y con el funcionamiento de la memoria en particular, teniendo en cuenta una doble disociación entre los cambios absolutos y relativos a eventos de las ondas theta y alpha. Esta doble disociación está caracterizada por un gran incremento en la potencia theta, durante un estado de reposo aparejado a un gran decremento en la potencia alpha, reflejan un buen funcionamiento de los procesos 
cognitivos y de memoria. El análisis de las ondas EEG en la banda delta y theta ha sido utilizado también para trabajar con adolescentes y averiguar cómo tienen lugar los procesos de maduración cerebral. Los ritmos delta y theta han sido ligados a los procesos de aprendizaje y a la plasticidad cerebral. Está ampliamente reconocido que los cambios que conducen a la maduración tienen lugar en el cerebro humano durante la adolescencia. Recientemente se realizó el primer estudio longitudinal [125] sobre la disminución de las señales EEG "non-rapid eye movement" (NREM) delta (1-4 Hz) y theta $(4-8 \mathrm{~Hz})$. Analizando dos veces al año las caracteristicas de la banda theta y delta de la señal EEG durante el sueño, en niños de 9 y 12 años, se observa cómo a partir de una edad concreta la densidad de potencia de la banda delta cae. Con la banda theta ocurre algo parecido, pero a una edad más temprana. Las conclusiones de este estudio sugieren que el intervalo de los 11 a los 16.5 años es el más importante en lo que a maduración para los procesos cerebrales se refiere. Este estudio es un ejemplo de que es posible utilizar la magnitud, la diferencia entre individuos y las caracteristicas adaptadas la edad de estos cambios de EEG como instrumentos excelentes no invasivos para investigar comportamientos neuronales que tienen correlación con la maduración del cerebro en la adolescencia.

Personas en estado despierto, no implicadas en procesamiento sensorial o en estado de toma de decisiones a nivel motor, desarrollan ritmos EEG 8-12Hz $(\mu)$ y $18-26 \mathrm{~Hz}$ $(\beta)$, sobre las áreas primarias de la corteza motosensorial. Estos ritmos EEG están bloqueados por movimientos activos y su representación mental. Por un lado, el decremento de la actividad oscilatoria en las bandas de frecuencia alfa y beta genera un fenómeno conocido como desincronización relativa a los eventos o ERD, generalmente producida por movimientos o preparación de movimientos. Por otro lado, tras el movimiento y en estado de relajación se producen ritmos EEG relacionados con el ERS o "Event Related Synchronization". Algunos sistemas BCI hacen uso del incremento o decremento de los ritmos Mu y Beta en las señales EEG durante las actividades motoras; estas respuestas son típicamente detectadas por análisis del espectro. El sujeto es entrenado para aprender a controlar sus ritmos mu y beta, principalmente a través de la imaginación de movimientos. Uno de los principales grupos de investigación en este tipo de sistemas BCI es el Wadsworth Center [126]. Diversos estudios desarrollados en este centro durante los últimos 14 años han mostrado que las perso- 
nas, independientemente que tengan o no discapacidades motoras, pueden aprender a controlar la ampitud de los ritmos cerebrales de la actividad electroencefalográfica sobre el cortex motosensorial y pueden usar dicha amplitud para, por ejemplo, controlar o mover un cursor en un entorno bidimensional [127]. En este experimento el sistema BCI está guiado por ritmos motosensoriales mu y beta, utilizando un cursor para realizar tareas en entornos de una y dos dimensiones. El procesado de señal que se realiza en este caso va desde el filtrado espacial hasta el análisis espectral pasando por la selección de canales. La traducción de características se realiza a través de un sistema de regresión y normalización. Las líneas de investigación actuales del Wadsworth Center se centran en aumentar la velocidad y la precisión de los sistemas BCI, tarea en la que también se centra la presente tesis doctoral. Otro de los centros de referencia es el "Graz center" [128] cuyas investigaciones han llegado a desarrollar sistemas pioneros que permiten a tetraplégicos controlar una silla de ruedas a través de señales electroencefalográficas [129]. El objetivo principal de este estudio fue demostrar que por primera vez las señales cerebrales podían ser utilizadas por tetraplégicos para controlar movimientos de su silla de ruedas en realidad virtual. En este caso, el sujeto que controlaba el BCI era un lesionado de columna vertebral, siendo capaz de generar oscilaciones en su electroencefalograma en banda beta a través de la imaginación de movimientos de sus pies paralizados. Estas oscilaciones beta fueron utilizadas para el control del dispositivo a través de un sistema BCI. De media, los participantes fueron capaces de desarrollar este experimento de forma asíncrona con una precisión del $90 \%$.

Tal y como afirman algunos autores [119], diversos desórdenes cerebrales pueden ser diagnosticados por la inspección visual de la señal EEG, fundamentalmente por aquellos expertos familiarizados con los cambios que podrían producirse en la señal EEG. La frecuencia y amplitud de las señales cambian de un estado a otro en el caso de adultos sanos dependiendo de la actividad que se esté ejerciendo. Un ejemplo de esto se muestra en la Figura 2.8, en la cual puede observarse el tipo de onda cerebral generado por un adulto sano en distintos estados de sueño y vigilia junto con los ritmos cerebrales asociados. La Figura 2.8 muestra registros EEG junto con señales electromiográficas registradas en los músculos y señales electrooculográficas (EOG) que registran el movimiento ocular. Los registros han sido obtenidos en distintas si- 


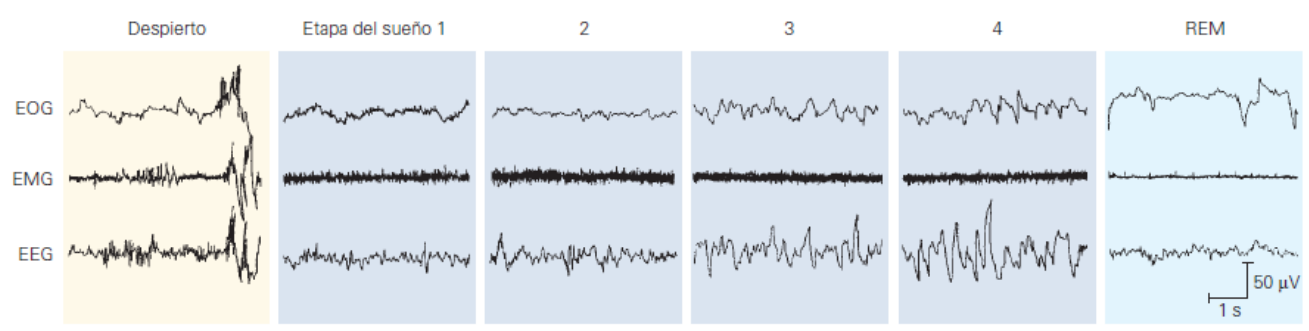

Figura 2.8: Diferentes estados del sujeto durante el registro simultaneo de señales electroencefalograficas (EEG), electromiográficas (EMG) que registra la actividad muscular y Electrooculograficas (EOG) que registra los movimientos oculares[130]

tuaciones. En primer lugar se han obtenido registros mientras el sujeto se encontraba despierto y consciente, y posteriormente se han obtenido registros en diferentes fases de sueño. Esto no solo ha permitido observar los distintos ritmos EEG, sino que también ha sido posible apreciar la correlación existente entre la señal cerebral, la señal muscular y el movimiento ocular en distintas situaciones.

Analizando las respuestas a eventos de la banda alpha, tetha y delta, algunos autores [117] han tratado de relacionarlas con el comportamiento de la banda gamma, fuertemente interconectada con las funciones sensoriales y cognitivas. De hecho, en este estudio se argumenta que las oscilaciones delta, theta, alpha y gamma pueden actuar como redes de comunicación resonantes para una gran población de neuronas. En este sentido se argumentaba que estos procesos oscilatorios podrían jugar un papel fundamental en la comunicación funcional dentro del cerebro relacionándose con la memoria y otras funciones de integración.

Hay evidencias suficientes para pensar que el ritmo mu y otros ritmos aplha son fenómenos independientes debido a las diferencias en la fuente de generación, sensibilidad a eventos sensoriales, coherencia bilateral, frecuencia y potencia. Los ritmos mu están presentes en una etapa muy temprana del desarrollo humano y de otras especies de mamíferos. Muestran capacidad de adaptación y propiedades para el cambio de forma dinámica. Sujetos individuales pueden aprender a controlar los ritmos mu en un corto espacio de tiempo. Recientes estudios han sugerido que los ritmos mu reflejan la modulación del cortex motor por las neuronas espejo prefrontales. Por ejemplo, células que podrian jugar un rol crítico en la imitación del aprendizaje y en la habilidad para entender algunas acciones. En este sentido, algunos autores [131] 
afirman que los ritmos mu representan una función importante en el procesado de información que une especificamente la percepción y la acción, la transformación de lo que el sujeto puede 'ver'y puede éscuchar én acciones concretras que puede 'hacer'. Otros autores [132] han estudiado las señales EEG sobre el área sensitiva-motora primaria y sobre el área premotora durante movimientos discretos de la mano y el pie. Se encontró desincronización relativa a eventos sobre el área primaria de la mano durante el movimiento del dedo y sobre el área primaria del pie durante el movimiento del dedo gordo del pie. A partir de estos resultados se podría pensar que cada área primaria sensitiva-motora tiene su propio ritmo intrínseco, el cual comienza a desincronzarse cuando el área correspondiente se activa. Se observó sincronización relativa a eventos, en forma de ritmo mu sobre electrodos que cubren el área primaria de la mano. Esto se observó no sólo durante el tratamiento visual, sino que también ocurrió durante el movimiento de pie. El área motora suplementaria también juega un rol importante en la preparación y planificación del movimiento. Ha sido demostrado que este área también muestra actividad rítmica dentro de la banda alpha, relacionado con el ritmo mu intrínseco del área primaria de la mano. Con la planificación y preparación del movimiento, el ritmo del área primaria suplementaria está desincronizado.

\subsubsection{Conclusiones}

Las señales EEG constituyen la firma de los procesos que tienen lugar a nivel cerebral. Estas señales electroencefalográficas pueden ser captadas a través de electrodos en el cuero cabelludo que monitorizan las señales eléctricas procedentes de la corteza cerebral mediante técnicas no invasivas. La corteza cerebral es la encargada de percibir, comprender e integrar los diferentes estímulos y en ella podemos analizar toda la actividad cerebral del movimiento voluntario así como la mayor parte de procesos cognitivos de una forma fiable. Tal y como se ha descrito en el presente capítulo, la mayoría de los trabajos de investigación en el campo del BCI está basado en señales electroencefalográficas. Esto es debido, entre otras razones, a la alta resolución temporal que presenta, a la facilidad de uso de estos sistemas, al bajo riesgo que genera para la salud del usuario y al bajo coste de los componentes. Sin embargo, en capítulos posteriores quedarán de manifiesto las limitaciones que hoy 
existen para el desarrollo de sistemas BCI de alta precisión con señales EEG. Estas barreras, como la baja relación señal a ruido, la vulnerabilidad ante perturbaciones fisiológicas y extrafisiológicas o las dificultades para localizar las fuentes de señal con precisión, están siendo superadas gracias al gran número de investigadores que trabajan en este campo. La mejora de estos sistemas debe estar focalizada en varios aspectos. En primer lugar debe optimizarse la calidad de la señal adquirida, mejorando tanto las condiciones de adquisición como los electrodos que captan la señal. $\mathrm{Y}$ en segundo lugar, es necesario mejorar el proceso por el cual se definen y detectan las características de la señal en los sistemas BCI. En este segundo punto, en los algoritmos y procesos a través de los cuales se analiza y clasifica la señal EEG, se centrará el siguiente capítulo.

Las conclusiones expuestas por los autores citados en este capítulo, demuestran que la señal EEG contiene información útil sobre procesos motores y cognitivos ejecutados por el sujeto. Extraer la información útil de la señal EEG constituye el principal reto de los sistemas BCI. 


\section{Capítulo 3}

\section{Análisis, procesado y clasificación de señales EEG}

¿Carreteras? A donde vamos no necesitamos carreteras.

Regreso al Futuro

\subsection{Introducción}

En el presente capítulo se abordan las principales características de los sistemas BCI, así como las técnicas de procesado de señal existentes para clasificar la señal EEG. Existen infinidad de algoritmos y métodos para decodificar y clasificar la señal EEG, por ello ha de analizarse la idoneidad de utilizar determinados algoritmos o métodos en función del tipo de sistema BCI a desarrollar. Será necesario analizar las características de la señal generada, así como las condiciones bajo las cuales funciona de forma óptima. Esto permitirá seleccionar el algoritmo más apropiado maximizando la precisión obtenida.

Para discutir y profundizar en los distintos tipos de sistemas BCI se utilizará como base el esquema propuesto en la Figura 3.1. En él se muestra el flujo de información en un sistema BCI típico [133], comenzando con la ejecución de una determinada tarea por parte del usuario mientras recibe un estímulo. Tras extraer las señales EEG, se procede a preprocesarlas, a extraer las características relevantes y a emitir una 


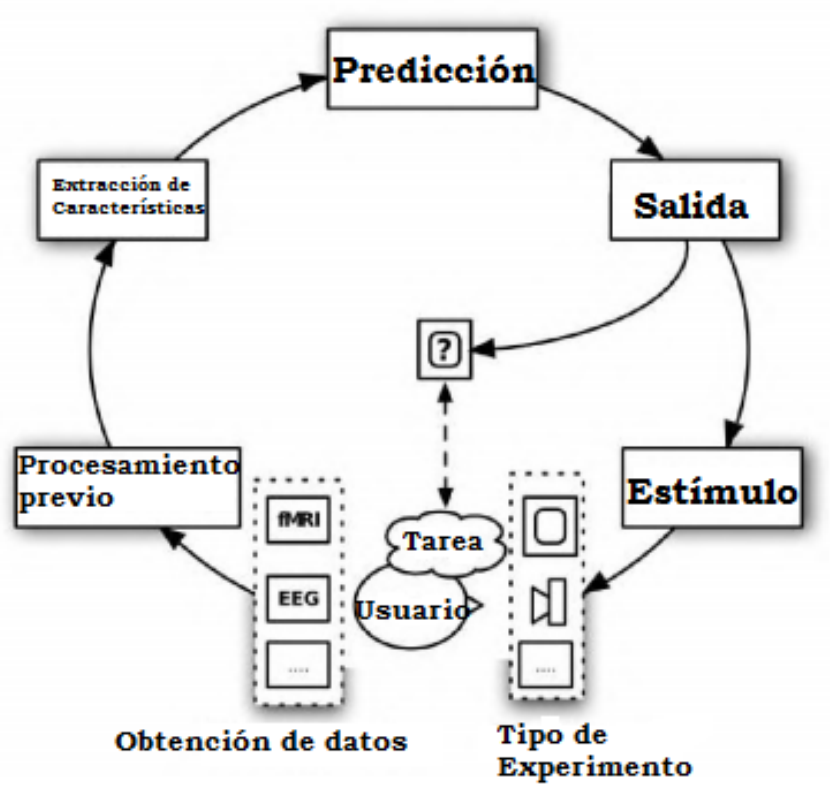

Figura 3.1: El ciclo del sistema BCI [133]

respuesta basada en la intención del usuario durante la captación de la señal EEG. Esta salida actúa como una señal de salida para controlar un dispositivo externo. El círculo se cierra cuando el usuario recibe la salida y se evalúa la precisión y comportamiento del sistema en función de la respuesta emitida. En este momento es posible ajustar el sistema o adaptar la actividad mental del usuario.

Debido a que el canal de comunicación BCI no depende ni de los nervios periféricos ni de los músculos, es posible utilizarlo en sujetos con graves problemas del sistema locomotor. Un sistema BCI permite que aquellos pacientes que están totalmente paralizados por esclerosis lateral amiotrófica, derrame cerebral o cualquier otra enfermedad neuromuscular puedan comunicarse con el entorno. Sin embargo, las aplicaciones prácticas de la tecnología BCI para satisfacer las necesidades de estos sujetos están limitadas por los requerimientos del estado del arte en la actualidad $[2]$.

Según algunos autores [133] la tarea ideal en un sistema BCI debe ser fácil de ejecutar, utilizando para ello el mínimo esfuerzo posible para prevenir la fatiga del sujeto. Debe generar grandes señales cerebrales para garantizar una interpretación rápida y veraz de las señales captadas. Sin embargo, no existe ninguna tarea que 
cumpla los requisitos anteriormente expuestos. Por ello, para maximizar la precisión en la decodificación de las señales EEG, se hace necesario evaluar los distintos tipos de sistemas BCI, los algoritmos disponibles para tratar la señal EEG captada y la forma en la que el usuario y el sistema BCI interaccionan durante la ejecución de una tarea motora o cognitiva.

A lo largo de los últimos años se han publicado estudios sobre los diferentes algoritmos de clasificación utilizados en investigaciones sobre el BCI basado en EEG, asi como sobre la identificación de las propiedades criticas de estos sistemas [134] [135]. Esto ha permitido obtener unas pautas claras que permiten seleccionar el algoritmo de clasificación mas apropiado en función del experimento BCI a realizar. En ocasiones, se realizan sesiones de calibración offline previas a la utilización del sistema BCI de forma online. Algunos estudios [136] han mostrado que las señales cerebrales utilizadas para controlar el sistema pueden cambiar de forma sustancial desde las sesiones de calibración offline hasta el control online, incluso con una sola sesión.

Las características de cada sistema BCI, los algoritmos de clasificación y la optimización de la precisión en la clasificación en función del objetivo de cada sistema, son los puntos fundamentales en los que se basa el presente capítulo.

\subsection{Tipos de sistemas BCI}

Los investigadores se han enfrentado a no pocas dificultades para comparar las tecnicas BCI descritas durante estos últimos años en la literatura cientifica. Como respuesta a esta situación, se ha venido trabajando para establecer métodos objetivos que permitan comparar entre sí tecnologias BCI distintas. Al tratarse de un campo de investigación muy joven, que se está desarrollando en un entorno multidisciplinar la comunidad BCI dispone de un lenguaje común. Sin embargo, no existe un modelo funcional común para describir un sistema BCI que posibilite la comparación de tecnologias BCI con otros sistemas BCI o interfaces de usuario no basadas en BCI. En este sentido, se ha propuesto en el pasado [137] la creación de este marco común que podría describir, evaluar y clasificar de forma efectiva todo tipo de sistemas y diseños BCI. Algunos autores han comenzado a clasificar los sistemas BCI en tres 
Capítulo 3. Análisis, procesado y clasificación de señales EEG

\begin{tabular}{ll}
\hline \hline $\begin{array}{l}\text { Sub-categoria del sis- } \\
\text { tema BCI }\end{array}$ & Descripción \\
\hline BCI Activo & Un sistema BCI activo es aquel cuya salida deri- \\
& va de la actividad cerebral generada bajo control \\
& consciente del usuario, independiente de eventos \\
& externos, con el propósito de controlar una aplica- \\
& ción o dispositivo
\end{tabular}

BCI Reactivo Un sistemas BCI reactivo es aquel cuya salida deriva de la actividad cerebral generada como respuesta a estimulación externa y es indirectamente modulada por el usuario, con el objetivo de controlar una aplicación o un dispositivo

BCI Pasivo Un sistema BCI pasivo es aquel cuya salida deriva de actividad cerebral arbitraria sin propósito de control voluntario y con el objetivo de enriquecer la interacción máquina-sujeto con información implícita

Cuadro 3.1: Clasificación de los sistemas BCI

sub-categorias: BCI activos, BCI reactivos y BCI pasivos. La descripción de cada uno de estos sistemas puede encontrarse en la Tabla 3.1.

\subsubsection{Sistemas sincronos $\mathrm{y}$ asincronos}

Desde el punto de vista de la sincronía del sistema BCI, es posible realizar una distinción clara entre los sistemas BCI síncronos y asíncronos. En el caso de los sistemas síncronos, un protocolo controla la duración de las operaciones así como el momento de inicio y fin. Es el caso de aquellos sistemas BCI en los que el sujeto es entrenado para mover un cursor en una dirección concreta: a izquierda o a derecha por ejemplo. Los movimientos imaginarios de la mano derecha desplazarían el cursor hacia la derecha del mismo modo que lo harían en dirección contraria en caso de imaginar movimientos con la mano izquierda. En este caso, el momento en el que el target aparece se informa al sujeto de que la tarea empieza y unos segundos después comienza a analizarse la señal EEG hasta que, una vez terminado el periodo de 
análisis el sistema toma la decisión de mover a un lado o a otro. En estos casos, suele ser frecuente que el sistema deje de analizar la señal EEG que se genera mientras la acción de movimiento, izquierda o derecha, es ejecutada. Por tanto, un sistema BCI síncrono, no permite al sujeto controlar el instante en el que una acción concreta debe o no ejecutarse, sino que reduce la posibilidad de ejecutar acciones a determinados periodos de tiempo durante los cuales la señal EEG será analizada y en base a la cual el sistema siempre tomará una decisión, esté usando un sujeto el sistema o no. Podemos encontrar multitud de ejemplos de experimentos que han utilizado sistemas BCI síncronos [138]. Por otro lado, los sistemas BCI asíncronos permanecen siempre activos mediante un análisis continuo de la señal EEG, reaccionando ante cualquier identificación positiva de un patrón de señal concreto asociado no sólo a acciones cognitivas o motoras, sino también ante la identificación del estado de reposo. Al igual que en el caso de los sistemas síncronos, existen multitud de experimentos recientes en los que se han utilizado sistemas BCI asíncrono [139] [140].

\subsubsection{Sistemas endógenos y exógenos}

La clasificación de los sistemas BCI se realiza en función del parámetro que tomemos como referencia. Si tomamos como base la naturaleza de la señal de entrada, podemos clasificar los sistemas BCI en sistemas endógenos y exógenos. Los sistemas BCI endógenos están basados en la habilidad del usuario para ejercer control directo y modular determinados parámetros de la actividad cerebral como la amplitud EEG dentro de un rango frecuencial determinado. Para conseguir un óptimo funcionamiento de estos sistemas es necesario un período de entrenamiento intensivo. Un ejemplo de sistemas endógenos son los sistemas BCI en potenciales corticales lentos (Slow Cortical Potentials - SCP). Los SCP son cambios lentos de voltaje generados sobre el córtex cerebral, con una duración variable entre 0.5 y 10 segundos. Los SCP negativos se asocian típicamente con el movimiento y otras funciones que implican una activación cortical. Se ha demostrado que las personas pueden aprender a controlar estos potenciales tras un proceso de aprendizaje. Este tipo de señales aparecen en la señal EEG debido a que también pueden producirse oscilaciones de menor duración que los ritmos EEG conocidos. Los SCPs han sido utilizados en sistemas BCI demostrando algunos estudios [141] que su autoregulación permitiría a un sujeto ha- 
bilitar un canal de comunicación verbal sin la necesidad de controlar ningún tipo de movimiento voluntario. La habilidad de los sujetos para controlar sus SCPs ha sido demostrada en diversos estudios que han constituido la base para los sistemas BCI conocidos como TTD (Thougth translation Device) [142]. Estos sistemas han sido ampliamente probados en personas con esclerosis lateral amiotrófica así como en personas sanas, habilitándoles mecanismos y capacidades de comunicación bien a través de navegadores web o dispositivos que permiten reconocer frases decodificando letra a letra. En los sistemas SCP, las sesiones de entrenamiento están basadas en retroalimentación visual, donde el sujeto puede monitorizar la negatividad/postitividad de las señales EEG, de esta forma los sujetos aprenden mecanismos para ajustar su actividad cerebral. El periodo de entrenamiento podría tardar semanas o meses, considerando sesiones de entrenamiento de 1 o 2 horas a la semana. Tras aprender la autoregulación de los SCP negativos, el funcionamiento de las tareas motoras y cognitivas se verá claramente mejorado. Algunos estudios han demostrado la eficacia de los SCP para establecer vías de comunicación en pacientes totalmente paralizados mediante el desarrollo de la habilidad de autoregular los SCP [143] [142]. Sin embargo, el principal problema de esta técnica proviene de la gran cantidad de tiempo de aprendizaje necesario para conseguir controlar y autoregular los SCP. En algunos casos, se ha conseguido autoregulación de los SCPs en un periodo de entrenamiento de 8 semanas SCP [141]. Los potenciales SCP también han sido utilizados en estudios recientes para analizar desórdenes neurológicos en niños, como el trastorno de déficit de atención e hiperactividad. A la vista de los resultados obtenidos [144], se podría afirmar que los niños que sufren este trastorno, podrían mejorar la habilidad para mantener la atención durante tiempo prolongado mediante el entrenamiento de los SCP.

Dentro de los sistemas BCI endógenos también cabe destacar los basados en imágenes motoras o ritmos sensoriomotores. Se basa en un paradigma de dos o más clases de imágenes motoras (movimiento de la mano derecha o izquierda, de los pies, de la lengua, etc.) u otras tareas mentales (rotación de un cubo, realización de cálculos aritméticos, etc.). Este tipo de tareas mentales producen cambios en la amplitud de los ritmos sensoriomotores $\mu(8-12 \mathrm{~Hz})$ y $\beta(16-24 \mathrm{~Hz})$, registrados sobre la zona somatosensorial y motora del córtex cerebral. Estos ritmos presentan 
variaciones tanto para la ejecución de un movimiento real como para la imaginación de un movimiento o la preparación al mismo. La imaginación motora engloba todos aquellos experimentos en los que se entrena al sujeto para controlar un sistema BCI mediante la imaginación de movimientos concretos. En este tipo de sistemas BCI, el sujeto es instruido para que imagine movimientos concretos frente a la pantalla de un ordenador con el objetivo de obtener los datos necesarios para diseñar el experimento en tiempo real. Este diseño se efectúa a partir de los datos EEG obtenidos y los estímulos presentados en la pantalla. La imaginación motora ha sido objeto de diversos estudios en el pasado [145], en algunos casos analizando los ritmos mu en la señal EEG y su asociación con la imaginación de movimientos de la mano derecha, mano izquierda, pie y lengua, demostrando que es posible utilizar un sistema BCI mediante imaginación de movimientos con múltiples clases.

Por el contrario los sistemas BCI exógenos basan su funcionamiento en la actividad electrofisiológica generada a partir de estímulos externos. Este tipo de sistemas, al contrario que los sistemas endógenos, no necesitan de una etapa intensiva de entrenamiento. Los sistemas BCI basados en potenciales evocados P300 o en potenciales evocados visuales de estado estable (Steady State Visual Evoked Potentials, SSVEP) son ejemplos de sistemas exógenos. Para traducir la señal EEG en acciones concretas, es posible analizar la señal desde distintos puntos de vista. Es comunmente aceptada [146] la existencia de señales de control contenidas en la actividad EEG a partir de cuyo análisis es posible desarrollar los sistemas BCI interpretando las intenciones del usuario. A partir de esta actividad cerebral, es posible detectar pequeñas alteraciones en el potencial eléctrico que aparecen tras un evento concreto, originado en el propio sujeto o en el exterior. Estos potenciales eléctricos, desencadenados como consecuencia de un evento, son conocidos como Potenciales Relacionados con Eventos (ERP). Debido a su pequeño valor en comparación con los valores que adquiere normalmente la señal EEG, han de utilizarse métodos de procesado de señal mediante la repetición de un mismo estímulo para poder extraer la respuesta de la señal EEG que la contiene. Dento de los ERP cabe destacar la onda P300 [44] que está siendo objeto de estudio desde hace varias décadas. La onda P300 puede describirse como una desviación positiva durante un ERP obtenido en humanos. Esta onda sólo ocurre si el sujeto está activamente implicado y concentrado en esta tarea. Según diversos estudios, su 
amplitud varía con el nivel de precisión del target, y su retardo varía con la dificultad de distinguir el estímulo objetivo de los estímulos estándar. Un retardo típico máximo cuando un sujeto hace una discriminación simple es de 300 milisegundos. Algunos autores [147] han demostrado recientemente que los pacientes con esclerosis múltiple sufren disfunciones cognitivas que pueden ser corroboradas analizando los cambios en la onda P300. Se demostró que aquellos sujetos con esclerosis múltiple han alterado la onda P300, reduciendo su amplitud y prolongando su retardo. Esta alteración ha sido utilizada como punto de partida por la comunidad científica para demostrar la existencia de disfunciones cognitivas en pacientes de esclerosis múltiple. La amplitud del P300 es directamente proporcial a la relevancia del estímulo que la genera. Dentro del grupo de los sistemas BCI con potenciales evocados, que será descrito posteriormente, se encuentran este tipo de sistemas BCI basados en la componente P300. Son señales generadas en la actividad EEG sobre el cortex parietal, que producen un evento infrecuente que puede ser auditivo, visual o somatosensorial, diferenciado de eventos frecuentes. El P300 es un componente positivo, generado después de 300ms de un evento infrecuente. El componente P300 comenzó a utilizarse en un sistema BCI hace más de dos décadas [148]. En estos casos el usuario se sitúa delante de un monitor con una matriz de MxM, con caracteres en cada uno de los elementos, donde cada columna y cada fila es iluminada alternativamente cada $125 \mathrm{~ms}$. El sistema hace la selección contando cuantas veces la columna o la fila contiene el carácter deseado que ha sido iluminado en función de los estímulos captados por el sistema EEG. Otros grupos han realizado estudios con el objetivo de mejorar el sistema descrito anteriormente [148], centrado su actividad en el desarrollo de nuevas aplicaciones, como el movimiento de un cursor de forma virtual desde un punto de partida hasta una localización deseada [44] en 2D o posibilitando la formación de palabras a través de la selección individual de los caracteres que la forman [8]. Si tras la aplicación de un estímulo visual, se analiza la señal EEG generada en la zona visual de la corteza cerebral, será posible detectar potenciales evocados visuales. Las señales procedentes de respuestas naturales a la estimulación visual a frecuencias concretas, los SSVEP. Estos tipos de señales cerebrales contienen una componente periódica que posee la misma frecuencia fundamental que la estimulación repetitiva, junto con una serie de armónicos secundarios. Por tanto, si el usuario fija su atención en una imagen que 
sufre un parpadeo a una frecuencia concreta, será posible detectar la frecuencia de parpadeo analizando las propiedades de la señal EEG generada debido a la relación directa entre el estímulo y la amplitud del SSVEP. Algunos autores [149] han realizado estudios para investigar en qué medida la precisión en la clasificación de un sistema BCI con 4 clases para clasificar, puede ser mejorada incorporando las oscilaciones de los armónicos evocados visualmente. Este estudio revela que el uso de tres armónicos partiendo de los SSVEP arrojan una mayor precisión en la decodificación muy significativa, en comparación con aquellos casos en los que se utilizan sólo dos armónicos. En este caso, la precisión obtenida por los distintos sujetos estaba entre $42.5 \%$ y $94.4 \%$. El potencial de los SSVEP está en continuo estudio, debido a las limitaciones en determinados usos. Por ejemplo, existen importantes limitaciones de los pacientes impedidos y con lesiones graves para la utilización de sistemas BCI basados en SSVEP debido a la necesidad de fijar la atención en los estímulos. De hecho, experimentos recientes [150] sugieren que es posible establecer comunicación en tiempo real a través de este tipo de sistemas, saltando las limitaciones impuestas por los SSVEP, todo ello de forma independiente de las funciones neuromusculares. A pesar de que en el pasado han existido tímidos avances en el diseño de un sistema BCI-SSVEP que supere las limitaciones anteriores, este no ha sido todavía probado con éxito en pacientes con lesiones cerebrales. La importancia de este estudio radica en que se propone un novedoso sistema BCI-SSVEP independiente y con tasas de clasificación mejoradas. Además, los resultados obtenidos tras el test en tiempo real, tanto en sujetos sanos como en pacientes con lesión cerebral grave, han arrojado precisiones de clasificación superiores al $80 \%$ en muchos casos. Por tanto, el potencial uso clínico de este sistema BCI tanto como herramienta de diagnóstico como para establecer comunicación con pacientes de lesiones cerebrales, deberá ser explorado en profundidad a partir de estos resultados. Recientemente ha cobrado especial interés la posibilidad de trasladar desde el laboratorio a la vida real, los avances en los sistemas BCI basados en SSVEP. Algunos autores han desarrollado estudios en este sentido [151], analizando la calidad de la señal obtenida mediante técnicas SSVEP a través de un sistema electroencefalográfico portatil. Además, estas señales fueron obtenidas mediante electrodos secos y comunicación inalámbrica, bajo condiciones desfavorables. Los resultados de este estudio demuestran que, mediante las 
técnicas adecuadas de análisis y detección de los SSVEP, es posible obtener buenos resultados que mejoren los sistemas BCI. Estos resultados constituyen una mejora considerable de las perspectivas de crecimiento de los sistemas BCI, ya que abren la puerta para la utilización de los sistemas BCI en entornos reales. Hasta ahora se han visto ejemplos en los que los sistemas BCI se han utilizado para interaccionar con un dispositivo externo seleccionando una de las opciones a través de la interpretación de las señales cerebrales. Sin embargo, según recientes estudios [152], en las últimas tres décadas ha incrementado considerablemente el interés de los sistemas BCI como herramienta para assistir, aumentar y rehabilitar las funciones sensitivas y motoras en los pacientes clínicos. Los sistemas BCI están siendo estudiados como elementos de ayuda en la terapia de rehabilitación para pacientes que han sufrido derrames cerebrales o lesiones con consecuencias similares. En particular, los sistemas BCI dedicados a la rehabilitación incorporan partes robóticas que manipulan de forma pasiva las extremidades afectadas. Estos sistemas detectan los eventos de sincronización/desincronización relativos a la actividad oscilatoria de los ritmos cerebrales asociados con la imaginación motora, encargados de controlar el sistema BCI por turnos. Evidencias previas sugieren que el proceso de imaginación motora activa preferentemente regiones sensoriales y motoras de forma similar a como ocurre con tareas realmente ejecutadas. Esta práctica repetida de imaginación motora puede inducir a cambios en la plasticidad del cerebro. Por tanto, tal y como sostiene [152], la combinación de la imaginería motora y los sistemas BCI podrían aumentar y mejorar la rehabilitación en pacientes que han sufrido derrame cerebral, mediante la activación de las redes corticomotoras a través de la imaginación motora, al tiempo que se suministra información sensorial de la extremidad afectada utilizando las partes robóticas.

\subsubsection{Dependientes e Independientes}

De lo expuesto anteriormente se infiere que un sistema BCI no utiliza los canales de salida normales del cerebro, tales como músculos o nervios para enviar comandos de control a una computadora. Sin embargo la actividad en estos canales de salida podría necesitarse como parte del proceso. Dependiendo de si el usuario utiliza la actividad de los músculos o nervios para producir la actividad cerebral, los sistemas 
BCI pueden clasificarse en dependientes o independientes. Un sistema BCI dependiente es aquel cuya información o comandos no son enviados a través de los canales de salida normales del cerebro, a pesar de que utilizan la información contenida en dichos canales para producir la actividad cerebral que contiene la información. Buenos ejemplos de este tipo de sistemas BCI dependientes son los VEP , o los BCI basados en SSVEP explicados anteriormente o los sistemas BCI basados en P300. Por otro lado, los sistemas BCI independientes no necesitan el soporte de los nervios periféricos o los músculos para producir los comandos para controlar la aplicación final. La mayoría de los sistemas BCI desarrollados suelen estar dentro de este último grupo de sistemas BCI independientes, como los sistemas basados en los ritmos motosensoriales y SCP [153].

\subsection{Análisis y clasificación de la señal en sistemas BCI}

La fiabilidad de un sistema BCI depende en gran medida de los procedimientos utilizados para la adquisición y procesamiento de las señales cerebrales. Para conocer la forma en la que cada una de las partes de un sistema BCI influyen en la precisión final del sistema, será necesario conocer el papel que desempeña cada parte del sistema dentro del ciclo BCI descrito anteriormente (Figura 3.1) [133].

La capacidad de los usuarios de los sistemas BCI para controlar ciertas características de la actividad EEG es una pieza clave para su buen funcionamiento. Por tanto, las condiciones fisicas, psicológicas y emocionales bajo las que el sujeto utilice los sistemas BCI condicionarán de forma clara los resultados obtenidos. Deben centrarse gran parte de los esfuerzos en la selección, detección y extracción de las características de la señal cerebral asociadas a una acción o estado mental concreto. Hace una década, algunos autores [154] ya se encontraban trabajando en diferentes métodos de extracción de características relevantes de la señal EEG para su utilización en sistemas BCI. Los resultados de estos estudios reforzaban la hipótesis de que las características seleccionadas reflejaban, al menos parcialmente, los aspectos independientes de los procesos corticales abriendo una nueva perspectiva para aumentar la eficacia de los sistemas BCI. Es posible concluir que la elección del algoritmo 
Capítulo 3. Análisis, procesado y clasificación de señales EEG

adecuado y su adaptación a las necesidades del sistema BCI es tan importante como la selección de las características relevantes de la señal EEG. Será esencial fijar claramente qué funciones desempeña el sistema, a qué objetivos esta orientado y qué tipo de señal EEG se desea procesar. Cada uno de los algoritmos utilizados en sistemas BCI poseen características específicas que aconsejan su uso bajo circunstancias concretas. Seleccionando el algoritmo correcto y las características adecuadas será posible optimizar la precisión total del sistema, tal y como quedará demostrado en capítulos posteriores.

\subsubsection{Detección, selección y extracción de características}

La selección de características ha sido objeto de investigación en infinidad de áreas de aplicación en las que se utilizan conjuntos de datos con decenas, cientos o miles de variables. Estas áreas incluyen el procesamiento de texto de documentos online, análisis de genes o química combinatoria. El objetivo de la selección de características tiene tres vertientes: Mejorar el funcionamiento de los sistemas de predicción, mejorar el sistema de predicción a través del aumento de la velocidad y reduciendo el coste computacional de la operación y mejorar el nivel de conocimiento sobre el proceso que ha generado los datos disponibles [155]. Por tanto, la selección de características es un aspecto fundamental en los sistemas BCI.

A lo largo de la historia del BCI han sido utilizadas una amplia variedad de características para diseñar sistemas BCI tales como los valores de amplitud de la señal EEG, la potencia de las bandas, los valores de potencia de la densidad espectral, parámetros autoregresivos o características de tiempo frecuencia. Tal y como algunos autores describían hace algunos años respecto al duseño de un sistema BCI [134], algunas propiedades criticas de estas características deben ser consideradas. En primer lugar debe tenerse en cuenta el ruido y los valores atípicos. Las características BCI son ruidosas o contienen valores atípicos debido a que las señales EEG suelen tener un nivel muy pobre de señal a ruido. Por otro lado hay que considerar también su alta dimensionalidad, ya que en los sistemas BCI, los vectores de características poseen un alto número de dimensiones. De hecho, muchas características son generalmente extraídas de algunos canales y de algunos segmentos de tiempo antes de ser concatenados en un único vector de características. También ha de tenerse en cuenta 


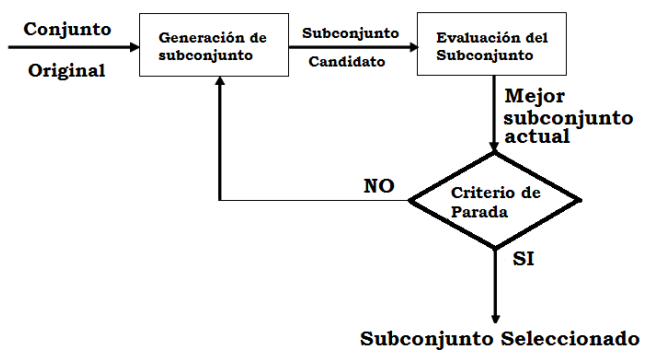

(a)

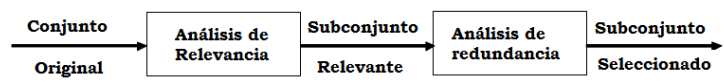

(b)

Figura 3.2: (a) Marco tradicional para la selección de características y (b) nuevo marco propuesto para la selección de características (adaptado de [156])

la información temporal. Las características BCI deben contener este timpo de información como los patrones de la actividad cerebral relativos a variaciones específicas de la señal EEG en el tiempo. Hay que considerar igualmente que las características BCI son no estacionarias, las señales EEG pueden variar rapidamente no solo de un instante a otro sino también y muy especialmente de una sesión a otra. Por último, hay que considerar también que los conjuntos de entrenamiento son relativamente pequeños, en tanto en cuanto los procesos de entrenamiento consumen y demandan tiempo del usuario del sistema BCI.

Algunos estudios [156] apuntan la necesidad de establecer nuevos métodos de selección de características. Se afirma que sería posible aumentar la eficiencia del proceso global en aquellos casos en los que se trate con datos de alta dimensionalidad. El aumento de la eficiencia del proceso de selección de características, pasa por la definición de la reduncancia de caracteristica asi como por la realización de un análisis explícito sobre este parámetro. En este estudio, se ha desarrollado un método basado en la correlación para el análisis de la relevancia y la redundancia. Además, se ha desarrollado un estudio empírico sobre la eficiencia y efectividad del modelo en comparación con otros métodos.

En esta línea, algunos autores [155] profundizaron en la importancia de los sistemas de selección y extracción de características. Esta necesidad venía generada por la evolución en el número de características que estaban siendo objeto de análisis en los conjuntos de datos o señales exploradas. En un primer momento, eran pocos los conjuntos de datos en los que se utilizaran más de 40 características. Esta 
situación ha cambiado de forma considerable en los últimos años y es frecuente encontrar experimentos en los que se analizan señales con cientos e incluso miles de características. Este hecho genera graves problemas en el análisis de la señal debido a la existencia de características que contienen información irrelevante o redundante. Este nuevo modelo de selección de características [156], descrito en la Figura 3.2b ha demostrado su efectividad bajo condiciones muy concretas. Los buenos resultados verificados por dos algoritmos diferentes de aprendizaje máquina, validaron la eficiencia y efectividad para la selección de características, tanto en casos de aprendizaje supervisado, como en aquellos dominios en los que los datos o señales analizadas contienen características irrelevantes o redundantes.

El éxito en el proceso de clasificación de un sistema BCI depende, en gran medida, del desarrollo correcto de la etapa de preprocesamiento de los datos. Debido a este hecho, un ámbito importante en las investigaciones sobre las tecnologías BCI está centrado en la extracción, selección y traducción de las distintas características de la señal EEG. Es necesario minimizar la verosimilitud de las interferencias de aquellas características que pueden ser generadas por otros procesos cognitivos. Estas interferencias pueden ser proyectadas en la corteza cerebral como ondas electroencefalográficas en una forma similar [28]. Por tanto, las características seleccionadas y asociadas a un proceso concreto deben ser fáciles de detectar utilizando métodos automáticos, reconocimiento de patrones, aprendizaje máquina, etc. En este sentido, es fundamental asegurar una buena relación señal a ruido, lo que puede conseguirse mediante aquellas señales que muestran una baja varianza, coherencia en los distintos intentos así como altas amplitudes tanto en el dominio del tiempo como en el de la frecuencia. La señal EEG captada será procesada para extraer características específicas de la señal, las cuales codifican los comandos generados en el cerebro del sujeto de una forma involuntaria o intencionada. Los métodos de extracción de características pueden también extraer información de la señal en el dominio del tiempo, como es el caso de las amplitudes de los potenciales evocados [157] o transformar la señal cerebral para que sea analizada en diferentes dominios como el dominio de la frecuencia o en el de tiempo-frecuencia. Una vez extraidas las características de la señal, éstas deben ser seleccionadas para maximizar la precisión del sistema. Las características extraidas para su uso en el sistema BCI aportarán mejor o peor separabilidad para 
las clases en función de muchos factores como la zona de la corteza cerebral en la que fue adquirida, el dominio en el que se encuentran, etc. Las características encontradas en el cortex motor, por lo general, aportan mejor separabilidad entre clases que en el caso de sistemas BCI basados en imaginería motora. También para sistemas BCI basados en la imaginación de movimientos, existen mayores posibilidades de que exista una mejor separación entre clases para las características en las bandas alfa y beta que para cualquier otra banda de frecuencia. Por tanto, no sólo es importante la extracción de características sino que también es muy necesario seleccionar las características adecuadas que serán utilizadas para la clasificación. Existen diversos métodos para la selección de características tales como R2 o algoritmos genéticos. En posteriores capítulos se describen los resultados obtenidos tras aplicar algoritmos genéticos como método para la selección de características. Una vez las características han sido extraidas y seleccionadas, es necesario clasificarlas. Existen multitud de procedimientos y algoritmos que han sido utilizados en experimentos BCI [158] [153] que pueden ser agrupados en lineales, como los clasificadores bayesianos, el análisis del discriminante lineal, etc., o no lineales como las redes neuronales.

Si se hace referencia a los algoritmos de clasificación de la señal en sistemas BCI, es necesario mencionar la adaptación de estos algoritmos a cada usuario y al contexto en el que se desarrollen. Los sistemas BCI, lejos de mantenerse en una configuración estática, están en coninua adaptación durante todo el proceso. La adaptación puede darse en tres niveles diferentes. En primer lugar, relacionado con el fenómeno neurofisiológico seleccionado para conducir el sistema BCI, el algoritmo de clasificación se orienta para adaptarse a las características producidas por este fenómeno. Si pensamos en el caso de SSVEP, el algoritmo de clasificación debe ajustarse para maximizar la detección de señales con amplitudes de frecuencia generadas por la estimulación visual [159]. El siguiente nivel de adaptación está relacionado con los factores que afectan a la generación de la señal EEG, entre los que se encuentran la fatiga, excitabilidad, enfermedad, etc. Estos factores pueden afectar de forma importante a la calidad de la señal EEG, por lo que el sistema de clasificación necesita un ajuste periódico a los posibles cambios producidos en las características de la señal EEG. Por último el tercer nivel de adaptación procede de la interacción entre el sistema BCI y el cerebro del usuario, ya que ambos son sistemas adaptativos en tanto en 
cuanto el sujeto aprenderá cómo usar características particulares para controlar el dispositivo de salida, con lo que el rendimiento mejorará con el uso prolongado. Es en este momento en el que el usuario asociará fenómenos neurofisiológicos concretos, generados espontanea o voluntariamente, con una tarea concreta. Por tanto, la adaptación pone el foco en la interacción entre el cerebro y el sistema BCI durante el proceso de aprendizaje, generando un reflejo en el cambio de las características de la señal así como en los resultados de la señal de salida, todo ello con consecuencias en la precisión final de la tarea. El algoritmo podría responder a un incremento de la precisión en la clasificación "premiando" al usuario con una comunicación más rápida o con tareas más complejas, reduciendo el tamaño de los targets, etc.

Desde una perspectiva abstracta, los algoritmos de clasificación seleccionan y transforman esa porción de datos EEG que mejor reflejan un determinado patrón de actividad cerebral previamente seleccionado. Este procedimiento se realiza normalmente en un proceso de tres pasos. La señal EEG genera una cantidad importante de datos que no transporta información sobre los procesos a nivel cerebral que están siendo investigados y deben ser filtrados para su eliminación. En el primer paso se aplicarán filtros restrictivos cuya consecuencia inmediata es la limitación de la señal EEG en términos temporales, espaciales o de ancho de banda oscilatorio [159]. Las características serán extraídas por el sistema basándose en el conocimiento derivado del campo de la neurociencia, utilizando herramientas para el tratamiento de señales. En este paso, las características particulares de la señal EEG serán seleccionadas, combinadas e intensificadas con procesamiento posterior [160]. Finalmente se procede a calibrar un algoritmo de clasificación basado en características prototípicas que son normalmente generadas en una sesión de entrenamiento a la que se somete al sujeto de forma separada. En este sentido, determinados estudios [161] han propuesto sistemas de entrenamiento basados en imaginación de movimientos que pueden ayudar al sujeto a obtener información sobre la señal generada, tras la imaginación del movimiento, mostrando en tiempo real el mapa de activación del cortex cerebral. Una vez entrenado el algoritmo, el sistema BCI será capaz de extraer información del estado cognitivo del usuario a partir de las señales EEG. Ha de destacarse que las investigaciones en el campo del BCI han desarrollado herramientas específicas para la extracción y selección óptima de las características [162] que se encuentran a 
disposición de los investigadores. El diseño de los algoritmos utilizados en sistemas BCI serán tratados en el capítulo siguiente. Diversos estudios [163] [153] [164] están siendo desarrollados con el objetivo de mejorar estas herramientas que constituyen uno de los aspectos clave para el rendimiento de cualquier sistema BCI.

En el pasado se han dedicado esfuerzos por parte de la comunidad cientifica con el objetivo de extraer aquellas características relevantes para la clasificación de la señal EEG durante la ejecución espontánea de tareas motoras [165]. Este estudio, ha demostrado empíricamente los beneficios que reportan la utilización de métodos de selección de características para la clasificación de tareas mentales mediante análisis de la señal EEG. Si tomamos como ejemplo el primero de los experimentos realizados, cada muestra EEG estaba representada por 138 características (6 canales con 23 componentes cada uno) y cada tarea ejecutada por el sujeto está compuesta por 40 muestras. En el segundo experimento la longitud de las secuencias y segmentos son de 1 y 0.5 segundos respectivamente. En este caso la resolución frecuencial es de 2 $\mathrm{Hz}$ y cada muestra EEG tiene 72 características (6 canales y 12 componentes cada uno). Este segundo experimento consta de un mínimo de 200 muestras por cada tarea. El trabajo se centra por tanto en el análisis de la relevancia de las distintas características, verificando si transportan información útil y relacionada con la tarea motora desarrollada por el sujeto. Aplicando los métodos desarrollados en dicho estudio, se consigue un doble objetivo: por un lado se seleccionan las características más relevantes y que contienen información útil, descartando aquellas características irrelevantes o con información redundante. Por otro lado se consigue información valiosa sobre la participación o no de las distintas partes de la corteza cerebral en tareas motoras. En este caso, de los resultados se desprende que los electrodos colocados en la línea media de la corteza, etiquetados como 'Cz'y 'Pz', son relevantes a la hora de reconocer tareas de imagineria motora izquierda y derecha, lo que se traduce en una participación de las áreas parietales en las tareas motoras, tal y como han enunciado algunos autores en el pasado [166]. Particularmente se muestra una mejora en el funcionamiento del clasificador para todos los sujetos considerados con solo una pequeña porción de las características iniciales. Mediante el uso de esas pocas características relevantes, no solo se ha conseguido incrementar la eficiencia de los sistemas BCI, sino que también se consigue un mayor nivel de adaptación del 
Capítulo 3. Análisis, procesado y clasificación de señales EEG

sistema BCI al usuario.

\subsubsection{Sistemas BCI espontáneos o de potenciales evocados}

Además de lo anterior, es posible diferenciar los sistemas BCI en dos grandes grupos en función de la forma en la que la señal cerebral es generada. En base a esto es posible hablar por un lado de sistemas en los que la señal analizada es evocada, como ocurre en el caso de los potenciales visuales evocados [167], y por otro lado de señal espontánea como el caso de potenciales corticales, ritmos motosensoriales o tareas cognitivas no motoras [168]. Dentro del primer grupo, es posible encontrar los sistemas BCI basados en SSVEP. Los potenciales visuales evocados pueden ser divididos a su vez en función de la amplitud en alta, media y baja frecuencia. Hace dos décadas tuvo lugar el primer intento de desarrollar un sistema BCI basado en potenciales visuales evocados [169]. La estimulación visual consistía, como en otros experimentos descritos anteriormente, en un número determinado de celdas etiquetadas que se iluminaban periódicamente en una pantalla. El mejor de los sujetos mostró una media de tiempo de respuesta de 1.5s por carácter, consiguiendo una precisión en la clasificación de aproximadamente el $90 \%$. Desde la publicación de los resultados de estas investigaciones, muchos investigadores han desarrollado sistemas BCI basados en potenciales visuales evocados centrándose en las posibilidades de extraer características de la señal o localizar la fuente principal de información útil [170], así como en la utilización de hardware con el propósito de simular la respuesta [50]. Los sistemas BCI basados en potenciales visuales evocados utilizan una pantalla donde se van mostrando una serie de objetos que se iluminan a diferentes frecuencias, cada uno de ellos etiquetado de forma diferente. Esto permite al sujeto relacionar cada uno de los objetos con una letra, aplicación o acción concreta. El tiempo de entrenamiento de este tipo de sistemas BCI es aceptable en comparación con otros sistemas como los basados en SCP [171] que requieren días e incluso semanas de entrenamiento. Por otro lado, los potenciales evocados somatosensoriales o "SSSEPs" han sido utilizados por algunos autores [172] dentro del objetivo común de todos los sistemas BCI de encontrar patrones cerebrales que sean fácilmente detectables. En concreto, el estudio anterior ha utilizado los SSSEPs como base para un sistema BCI que utiliza transductores para estimular los dedos índices de ambas manos a 
través de la estimulación táctil, en el rango de frecuencia de resonancia del sistema somatosensorial. La estimulación vibratoria es reflejada en la señal EEG como SSSP, con lo que posibilita que el sujeto module su actividad cerebral concentrando su atención en el dedo izquierdo o el derecho. El primer paso para desarrollar estos sistemas debe comenzar determinando la frecuencia de resonancia específica del sujeto, que será la frecuencia óptima de la estimulación somatosensorial. Los sujetos serán entonces estimulados con su frecuencia específica de estimulación en el dedo índice derecho, escogiendo una frecuencia diferente para el dedo índice de la mano izquierda. La estimulación se realizará de tal forma que el sujeto creerá que ambas son iguales. Las características de los electrodos etiquetados como C3 y C4 podrían ser extraidas en el dominio del tiempo, la cual puede ser posteriormente promediada a través de un periodo de tiempo de un segundo. En este caso se utiliza el algoritmo de análisis del discriminante lineal (LDA). Según el estudio, dos de cuatro sujetos aprendieron a modificar su SSSEPs para controlar un sistema BCI de dos clases con una precisión de entre el $70 \%$ y el $80 \%$. En los últimos años ha crecido el interés por el uso de SSVEP en los sistemas BCI. Este enfoque es uno de los más rápidos y fiables para la comunicación aplicando sistemas no invasivos. Sin embargo, es necesario mejorar la velocidad en términos de tasa de transferencia de información y el tiempo necesario para realizar un único comando.

\subsubsection{Sistemas BCI online y offline}

Existen dos vias para procesar los datos EEG y utilizarlos en sistemas BCI [28]. El análisis offline es útil para conocer qué información podría ser extraída en el conjunto de datos obtenidos y qué vias podrían ser las mejores para extraer dicha información. Este análisis es muy útil para realizar el ajuste de los algoritmos de detección durante la ejecución de una determinada tarea por parte del sujeto. Segun la bibliografía [28], el análisis offline permite extraer conclusiones sobre tres aspectos distintos del sistema BCI analizado. En primer lugar, este análisis permite analizar los datos obtenidos y relacionarlos con mecanismos y conocimiento ya validado con base en neurociencia. En segundo lugar, se detectan patrones elegidos en los datos obtenidos, con la esperanza de poder detectar estos patrones en nuevos conjuntos de datos obtenidos en el mismo contexto. Por último, el resultado del análisis offline, permite 
extraer conclusiones sobre el rendimiento esperado del sistema BCI. La validez de estos tres aspectos del análisis offline depende del procedimiento seguido para realizar el análisis y de la porción de datos utilizados en el proceso.

Algunos autores [43] han descrito un nuevo tipo de sistema BCI que utiliza un prototipado rápido utilizando herramientas como matlab o Simulink. En dicho trabajo, los autores automatizan la transición entre los experimentos online y offline para el caso de experimentos en tiempo real. Este sistema es capaz de procesar múltiples canales EEG online y operar en tiempo real en un PC estandar. Los resultados arrojan un buen resultado en términos de precisión de clasificación, obteniendo entre un $70 \%$ y un $95 \%$ con dos canales EEG después de algunas sesiones con retroalimentación utilizando un modelo autorregresivo y análisis del discriminante lineal (LDA).

El objetivo de todo análisis offline es tratar de imitar el análisis online tanto como sea posible. Esto ocurre en los análisis causales, que siguen tres reglas básicas: En primer lugar solo tienen en cuenta datos que realmente están disponibles en el punto temporal que se desea analizar. Esto es especialmente relevante para métodos de filtrado que de forma intherente podrían acceder a "datos futuros" (como en el caso del filtrado adelante-atrás). En segundo lugar debe tenerse en cuenta que el tiempo de procesamiento de los algoritmos influye de forma directa en la aplicabilidad del sistema en tiempo real. El hardware y software utilizado también influirá de forma decisiva en el rendimiento del sistema en tiempo real. En tercer lugar, ha de garantizarse la disponibilidad de los datos y la información procesada no solo en el escenario offline sino muy especialmente en el escenario online.

Algunos autores [173] han realizado experimentos utilizando análisis offline de los datos recogidos durante la operación del sistema para explorar métodos que pudieran mejorar la precisión de la clasificación. Los datos fueron aglutinados durante la selección de los usuarios de entre tres posibles targets mediante control vertical del movimiento del cursor. Los tres métodos analizados difieren en la dimensionalidad del movimiento del cursor y en el tipo de función utilizada (lineal vs no lineal). 


\subsection{Algoritmos de Clasificación en sistemas BCI}

La literatura especializada describe los cambios experimentados por los sistemas BCI desde sus planteamientos iniciales [28]. En un primer momento, los sistemas BCI estaban basados en los esfuerzos realizados por el usuario para generar patrones en la señal cerebral, que pudieran ser reconocidos facilmente. Sin embargo, la evolución de los sistemas BCI surgida tras el desarrollo de las técnicas de aprendizaje máquina han hecho que el esfuerzo de aprendizaje se desplace desde el usuario hacia los algoritmos implementados en el sistema BCI. El aprendizaje máquina es una parte de las ciencias de la computación que se ocupa del desarrollo e implementación de sistemas capaces de generalizar patrones de funcionamiento, partiendo de información muestral o procedimental sobre un proceso concreto. Como se verá a continuación, este campo esta muy relacionado con la estadística y el análisis de datos. El aprendizaje máquina tiene aplicación en infinidad de casos prácticos, y durante estos años ha sido aplicado a la detección de vertidos de petróleo en imágenes captada por satélites [174], en los procesos de clasificación automática del tráfico de red [175] e incluso en procesos de diagnóstico en el campo de la medicina [176].

Algunos autores [28] han tratado de describir los aspectos básicos del aprendizaje maquina aplicado al BCI. Los patrones en la actividad EEG son generados tras la ejecución mental de ciertas tareas, las cuales han sido aprendidas a una edad temprana de la vida del sujeto. El usuario genera inicialmente "prototipos" de datos que son utilizados para calibrar el algoritmo de aprendizaje. En este caso, los datos EEG son procesados y analizados en tiempo real para detectar los patrones aprendidos previamente e interpretarlos como comandos. Esto puede ser utilizado para discriminar la presencia o ausencia de distintas clases que pueden ser vistas por el sistema de clasificación como estados cognitivos especificos. El resultado de este análisis es una clasificación de los datos introducidos (ver Figura 3.3), que usualmente se asigna a un valor numérico o a clases discretas predeterminadas. En la última decada, numerosos investigadores se han centrado tanto en el desarrollo de métodos novedosos de aprendizaje máquina para el desarrollo del BCI, como en la mejora de los métodos existentes [177] [178].

La selección del algoritmo más apropiado para un experimento BCI condicionará en gran medida los resultados obtenidos. Es obvio que no todos los algoritmos 


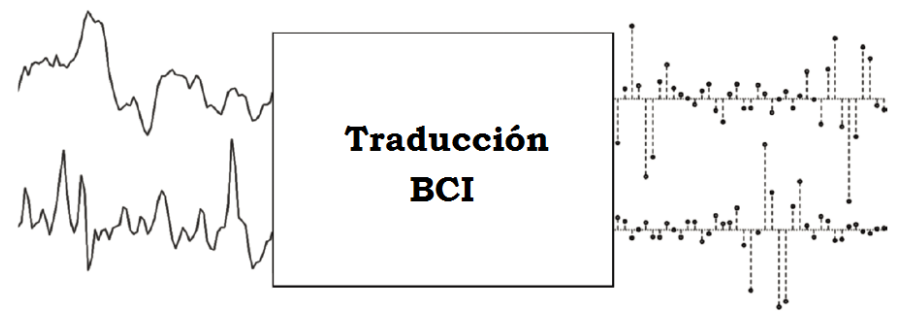

Figura 3.3: Un sistema BCI traduce la señal de entrada EEG en estimaciones de estados cognitivos (adaptado de [28])
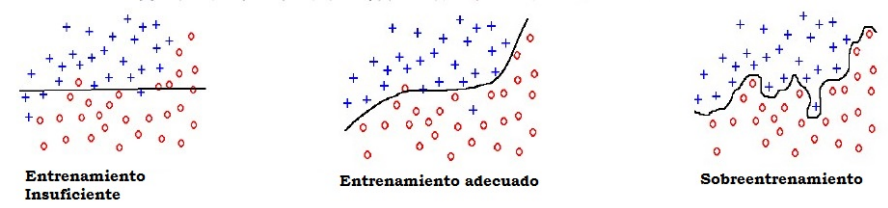

Figura 3.4: Fronteras de decisión obtenidas tras un entrenamiento insuficiente (1), tras un entrenamiento adecuado (2) o en el caso de un algoritmo afectado de sobreentrenamiento (3)

de clasificación arrojarán los mismos resultados para un mismo sistema BCI. Por ello, han de analizarse claramente los puntos fuertes y los puntos débiles de cada uno de los algoritmos de clasificación disponibles. Disponer de esta información permitirá optimizar el proceso de clasificación, utilizando el algoritmo que sea capaz de diferenciar mejor entre las distintas clases que deban ser clasificadas durante el proceso. Será esencial entender claramente todos estos aspectos, considerando también las variaciones en el tiempo de la señal EEG. A continuación se describen los principales algoritmos de clasificación utilizados durante los últimos años en los sistemas BCI, junto con las principales características asociadas a cada uno de ellos.

Según la literatura cientifica [134], se han utilizado distintas definiciones para describir los diferentes tipos de algoritmos de clasificación. En primer lugar se mencionan los Generativos-discriminativos. Los clasificadores generativos, también llamados informativos, computan la verosimilitud de cada clase y seleccionan aquella con mayor valor. Los clasificadores discriminativos, como las SVM (Support Vector Machines), solo aprenden la forma de discriminar las clases con el objetivo de clasificar un vector de características directamente. Los clasificadores estáticos, como los perceptrón multicapa, no pueden tener en cuenta la información temporal duran- 
te la clasificación, ya que utilizan para la clasificación un vector de características individual. Por otro lado, los clasificadores dinámicos, como los modelos ocultos de markov o "Hidden Markov Model", pueden clasificar una secuencia de vectores de características y conseguir asi captar las dinámicas temporales. Mención especial merecen los clasificadores estables y no estables. Los clasificadores estables, como el análisis de discriminante lineal o LDA, tienen una baja complejidad. Se denominan estables debido a que las pequeñas variaciones en el conjunto de entrenamiento no afectan de forma considerable a su fincionamiento. Por el contrario, los clasificadores no estables como el perceptrón multicapa, tienen una gran complejidad y pequeñas variaciones en el conjunto de entrenamiento pueden generar importantes cambios en su funcionamiento. Utilizando algoritmos regularizados, es posible añadir robustez al sistema. La regularización consiste en controlar cuidadosamente la complejidad de un clasificador con el objetivo de prevenir el sobreentrenamiento. Para poner en marcha un algoritmo de clasificación se suelen utilizar dos tipos de subconjuntos extraidos del conjunto de datos disponibles: El conjunto de entrenamiento y el conjunto de prueba. El conjunto de entrenamiento es utilizado para calibrar el algoritmo de clasificación y obtener determinados parámetros de clasificación. A continuación, se utiliza el conjunto de test para validar el algoritmo y evaluar el error de generalización arrojado por el algoritmo de clasificación. El conjunto de entrenamiento y el conjunto de test no deben contener muestras en común, ya que no tendria sentido evaluar la calidad del modelo sobre los datos que han servico para construirlo (Figura 3.4). En caso de que ocurriese esto último, se obtendrían resultados demasiado optimistas no correspondientes con la capacidad real del algoritmo para clasificar correctamente. La pérdida de la capacidad de generalización es una de las consecuencias del sobreentrenamiento. No existe un criterio único para dividir el conjunto inicial en datos de entrenamiento y de test, sin embargo algunos autores [179] afirman que de forma orientativa se deberia utilizar un $80 \%$ de los datos para el conjunto de entrenamiento y un $20 \%$ para el conjunto de test y validación que permita estimar la generalización. Un clasificador regularizado tiene una buena capacidad de generalización y es muy robusto respecto a los valores atípicos (outliers).

Existen muchas formas de categorizar los algoritmos de clasificación utilizados en sistemas BCI. Algunos autores [134] proponen dividir estos algoritmos en varias 
Capítulo 3. Análisis, procesado y clasificación de señales EEG

categorías: Clasificadores lineales, redes neuronales, clasificadores bayesianos y vecino más cercano.

\section{Clasificadores lineales}

Los clasificadores lineales son algoritmos de clasificación que utilizan funciones lineales para crear fronteras de separación entre las distintas clases del problema planteado. Dentro de este tipo de algoritmos de clasificación, pueden distinguirse dos tipos de clasificadores lineales para aplicar a los sistemas BCI. Por un lado se encuentran los algoritmos LDA o análisis del discriminante lineal. Según algunos autores [180], la utilización de clasificadores lineales en investigaciones BCI es algo muy extentedido. La clasificación lineal utiliza un modelo muy simple y por ello si no se cumplen una serie de condiciones, este tipo de clasificadores pueden verse condicionados negativamente. La existencia de datos atípicos o la presencia de señales demasiado ruidosas pueden generar problemas de clasificación y bajo rendimiento utilizando clasificadores lineales. Lamentablemente, es común encontrar estos dos factores en el análisis de las señales utilizadas por sistemas BCI. El algoritmo LDA fue propuesto por Fisher [181] hace más de 70 años. Esta técnica, divide el espacio muestral en subespacios mediante hiperplanos que permiten optimizar la separación entre los conjuntos objeto de clasificación. El algoritmo LDA busca maximizar la distancia entre clases mientras minimiza la distancia entre las muestras que pertenecen a la misma clase. Dicho de otra forma, LDA realiza una transformación lineal que minimiza la dispersión dentro de cada clase del conjunto de entrenamiento mientras maximiza la dispersión entre las clases [182]. La Figura 3.5 ilustra el modo de funcionamiento del algoritmo LDA. Partiendo de un conjunto de entrenamiento con tres clases diferentes (Figura 3.5a), se genera una proyección del conjunto de entrenamiento (Figura 3.5b), minimizando la dispersión dentro de cada clase y consiguiendo que la distancia entre las observaciones medias de cada una de las clases y la media global obtenida sea máxima (Figura 3.5c). La bibliografia contiene ejemplos del proceso de mejora que ha sufrido este algoritmo desde sus inicios por parte de la comunidad científica [183]. Las mejoras introducidas por los investigadores han estado orientadas a la eliminación de restricciones que planteaba el modelo LDA inicial. Gracias a estas mejoras se ha podido encontrar una función discriminante 


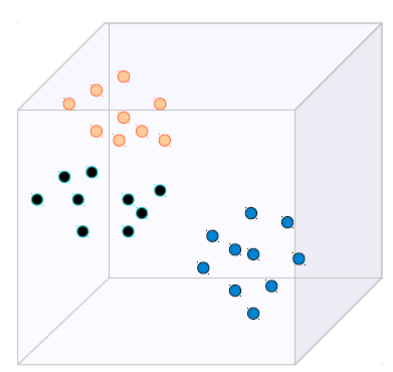

(a)

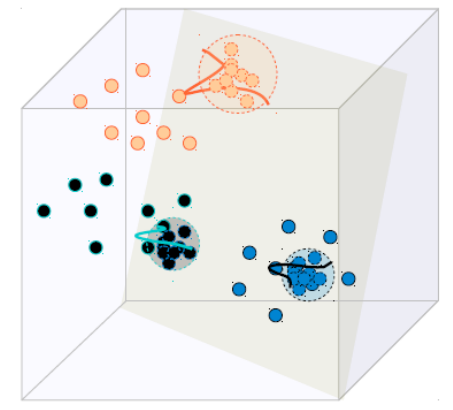

(b)

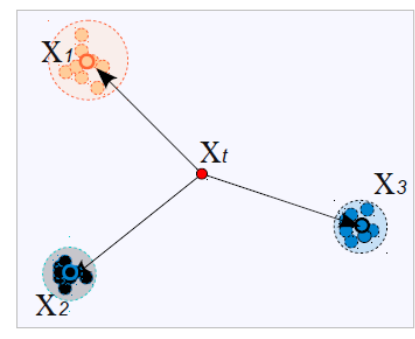

(c)

Figura 3.5: Modelo de funcionamiento del algoritmo LDA [182]

para utilizar en aquellos conjuntos de datos en los que no es posible tener igualdad de matrices de covarianzas [184] o evitar que los datos atípicos pudieran afectar al proceso de clasificación contaminando el proceso de obtención de estimadores. Para ello se acabó proponiendo un nuevo modelo que permita la detección y eliminación de estas observaciones atípicas evitando que afectaran al proceso de clasificación. Esto último fue posible gracias a la introducción de la estimación "high breackdown" [185], produciendo estimadores inmunes a la distorsión causada por una minoria de datos u observaciones atipicas.

Los métodos lineales asumen que es posible una separación lineal de los datos y que estos están conformados en una distribución normal. Estudios recientes sobre técnicas de reconocimiento de patrones para la clasificación EEG en sistemas BCI han puesto de manifiesto el potencial del algoritmo LDA en estos entornos [49]. Los clasificadores lineales son generalmente más robustos que sus clasificadores homólogos no lineales. Este hecho se fundamenta en la flexibilidad limitada de los clasificadores lineales (menos parámetros para definir) y son por tanto menos proclives a generar sobreentrenamiento. Sin embargo, en presencia de fuerte ruido y datos atípicos, también los sistemas lineales pueden resultar poco precisos. Tanto la Figura 3.6b como la Figura 3.6c muestran que ante la presencia de fuerte ruido y datos atípicos, se pueden generar cambios drásticos en la frontera de decisión. Esto ocurrirá si no se limita la influencia de determinados datos durante el proceso de entrenamiento. Para evitar y controlar los problemas generados durante el entrenamiento es posible 


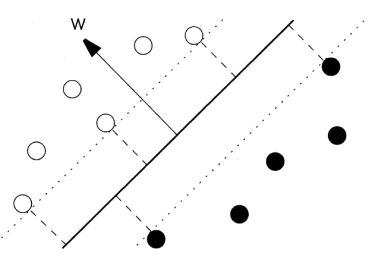

(a)

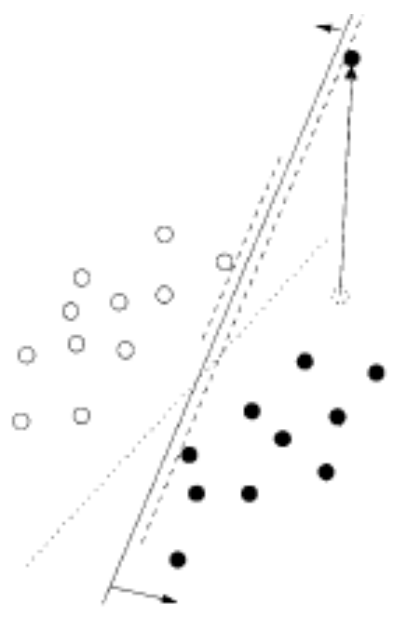

(b)

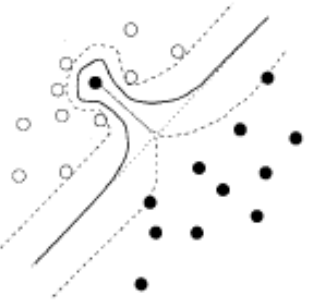

(c)

Figura 3.6: (a) un clasificador lineal está definido por el vector normal al hiperplano 'W'y un offset. El margen de un clasificador lineal es la distancia mínima desde los puntos de entrenamiento hasta el hiperplano. El margen está representado por la distancia entre las líneas punteadas y la línea sólida. (b) Conjunto de datos con presencia de datos atípicos. (c) Conjunto de datos con una etiqueta incorrecta ([180])

utilizar regularización. Aplicando regularización sería posible limitar la influencia de los datos atípicos y reducir la complejidad del clasificador [180].

Además de todo lo expuesto, ha de tenerse en cuenta las características de los conjuntos de datos que toman parte en los experimentos BCI. En este sentido, la utilización del algoritmo LDA en experimentos BCI puede generar problebas relacionados con el la utilización de un pequeño número de muestras (small sample size problem), descrito en detalle por la bibliografía científica [186]. Esto ocurre cuando solo hay disponibles $m$ muestras en un espacio vectorial $n$-dimensional con $m<n$. Dadas las matrices de dispersión entre clases $S_{B}$ e intra clases $S_{W}$, las consecuencias de este hecho hacen que la matriz de dispersión intra clase, dada por $S_{W}=\sum_{j=1}^{c} \sum_{i=1}^{m_{j}}\left(x_{j}^{i}-x_{j}\right)\left(x_{j}^{i}-x_{j}\right)^{T}$ sea singular y no tenga inversa. Este hecho, genera un problema grave en la obtención de una solución a partir del método LDA ya que es necesario que la matriz $S_{w}$ (matriz entre clases: sea no singular, pudiéndose obtener de esta forma los vectores propios de $S_{w} * S_{b}^{T}$ a fin de obtener la matriz de proyección maximizando la ecuación $W_{l d a}=\operatorname{argmax}_{\|w\|=1} \frac{W^{T} S_{B} W}{W^{T} S_{W} W}$. Para resolver este problema generado por un tamaño de muestras pequeño en comparación con el 
número de dimensiones, una de las opciones utilizada con éxito en el pasado ha sido utilizar técnicas de regularización [187]. Algunos autores [187] han propuesto un nuevo modelo de análisis discriminante regularizado (RLDA) para resolver el problema del tamaño de muestras pequeño en experimentos de reconocimiento de rostros. Los resultados de este experimento confirman la efectividad de las técnicas de regularización para solventar el problema planteado. Las tecnicas de regularización fueron propuestas en 1989 por Friedman [188]. Este método de análisis del discriminante regularizado se basa en la introducción de un parámetro al estimador de la matriz de covarianza, lo que consigue eliminar gran parte de las limitaciones del algoritmo original.

Algunos autores que han introducido mejoras en el algoritmo RLDA [189], han descrito las limitaciones que presenta el algoritmo LDA. El algoritmo LDA es un método de clasificación bien conocido que proyecta datos con alta dimensionalidad en un espacio de baja dimensionalidad donde los datos son reformateados para maximizar la separabilidad de las clases. La proyección óptima en el algoritmo LDA clásico es obtenido minimizando la distancia de las muestras dentro de una misma clase al tiempo que se maximiza la distancia entre muestras de distintas clases, asi se obtiene la máxima discriminación. El algoritmo LDA clásico utiliza tres matrices de dispersión: La matriz intra clase $S_{i}$, la matriz entre clase $S_{w}$ y la matriz total de dispersión $S_{t}$. La matriz total de dispersión es un múltiplo de la matriz de covarianza y debe ser no singular. Sin embargo en algunas aplicaciones, como clasificación de texto o reconocimiento facial, todas las matrices de dispersión en cuestión pueden ser singulares, debido a que los datos están en un espacio con gran número de dimensiones y el tamaño de las muestras no excede dicha dimensión. En estas situaciones se genera el conocido como problema de la singularidad. Estos autores afirman que RLDA aporta una solución efectiva para el problema de la singularidad. La idea general de este algoritmo es la de añadir una constante $\lambda>0$ a los elementos diagonales de la matriz total de dispersión; este será el parámetro de regularización. La regularización estabiliza la estimación de las muestras de la matriz de covarianza y mejora el funcionamiento de la clasificación del algoritmo LDA. Tal y como ya afirmaba Friedman [188], escoger un valor apropiado para el parámetro de regularización es crítico para el buen funcionamiento de RLDA. Un valor grande de $\lambda$ puede generar distorsiones 
en la información de la matriz de dispersión. Un valor pequeño de $\lambda$ puede no ser suficientemente efectivo para resolver el problema de la singularidad. $\lambda$ variará entre $0 \leq \lambda \leq 1$. Será el encargado de regular la complejidad del clasificador, produciendo una mezcla entre el caso de la regla de discriminación lineal y cuadratica. Por tanto, la matriz de covarianza estimada será ahora una mezcla de la covarianza de las muestras de cada clase y la covarianza común de los datos. La estimación obtenida será la que se emplee en la regla de decisión de verosimilitud gausiana. El parámetro $\lambda$ será determinado en función del error de clasificación, que se obtendrá para cada valor propuesto en cada uno de los parámetros [190]. El valor óptimo para $\lambda$ no tiene por qué ser conocido de antemano. Se debe tener un método de entrenamiento basado en el conjunto de muestras para estimarlo. Este hecho es un objetivo común asociado a todos los métodos de regularización. Para determinar el valor del parámetro de regularización de forma óptima, el método más utilizado es el de validación cruzada (cross-validation) [191] [192]. Para estimar los parámetros en este contexto de clasificación, también cabe la posibilidad de utilizar el método "bootstrapping". Algunos autores [193] han descrito y comparado en el pasado la validación cruzada y el "bottstrapping", los dos métodos más comunes para la estimación de la precisión en este contexto. Los resultados experimentales, utilizando conjuntos de datos de grandes dimensiones, indican que el método con el que se obtienen mejores resultados es el de validación cruzada utilizando diez "folds. ${ }^{0}$ rodajas. Este sigue siendo el método que mejores resultados arroja, aún cuando la capacidad computacional pudiera permitir la utilización de un número mayor de rodajas. La validación cruzada divide los datos disponibles de forma aleatoria en un conjunto de entrenamiento y un conjunto de test. El conjunto de entrenamiento se subdivide también en dos conjuntos: por un lado el conjunto de estimación que se utiliza para seleccionar el parámetro y por otro lado el conjunto de validación, utilizado para validar el rendimiento del algoritmo. La validación cruzada de K-particiones (k-fold cross validation) es la más utilizada en la actualidad y consiste en dividir el conjunto de ejemplos disponibles en 'k'conjuntos de igual tamaño $T_{1}, T_{2}, \ldots, T_{k}$. En esta validación cruzada se realizan $k$ experimentos, utilizando $T_{j}$ (con $j \neq i$ ) como conjunto de entrenamiento en la iteración i-ésima, y $T_{i}$ como conjunto de test. El esquema de funcionamiento de la validación cruzada en k-particiones está descrito en la Figura 3.7. Una de las principales ventajas 
de este diseño es que las estimaciones del error sobre los conjuntos de test son independientes, debido a que los conjuntos de test no se solapan [192]. Aplicando la validación cruzada es posible comprobar los resultados obtenidos para distintos valores del parámetro de regularización sobre distintos conjuntos de test. Escoger el valor más apropiado de los parámetros de regularización para cada situación particular es el objetivo de este proceso. Cuando se produzcan situaciones favorables a la aplicación del algoritmo de regularización, el procedimiento deberá escoger un alto grado de regularización que reducirá sustancialmente la varianza, al tiempo que introduce una pequeña tendencia extra, reduciendo de forma importante el riesgo de errores en la clasificación. Por otro lado, cuando la situación no aconseja aplicar regularización, hay posibilidad de que el proceso de selección de parámetros pueda producir un pequeño grado de regularización [188]. La propuesta de Friedmann trata de elegir valores de la matriz de covarianza conjuntamente, tanto del parámetro de regularización $\lambda$ como de $\gamma$, que minimicen el error futuro de clasificación en el proceso de validación cruzada. El potencial del algoritmo LDA regularizado, o RLDA, para mejorar el error de clasificación, dependerá de la distribución de la población y del tamaño de las muestras. En aquellas situaciones en las que el tamaño de las muestras de clases $N_{k}$ sean más grandes que la dimensión del espacio de medida $p$, no será necesaria regularización y el proceso de selección de parámetros tenderá a producir valores pequeños de $\lambda$. En aquellas situaciones en las que se dispone de un número reducido de muestras o cuando las matrices de covarianza de las de las clases son muy diferentes y/o no demasiado elipsoidales la aplicación del algoritmo RLDA producirá buenos resultados. Por el contrario, situaciones en las que las matrices de covarianza de cada clase son todas iguales y altamente elipsoidales generan grandes dificultades para un buen funcionamiento del algoritmo RLDA. Por otro lado, cuando el tamaño de la muestra es suficientemente pequeña como para que no sea posible obtener buenos resultados con LDA, la regularización aportada por RLDA es la única esperanza. Este clasificador con regularización introduce un parámetro que puede permitir o castigar errores de clasificación en el conjunto de entrenamiento para construir una regla general. El sistema de clasificación construido a partir de este algoritmo posee una mayor capacidad de generalización respecto de otros algoritmos en los que no se utiliza regularización. Tal y como se ha comentado anteriormente, 


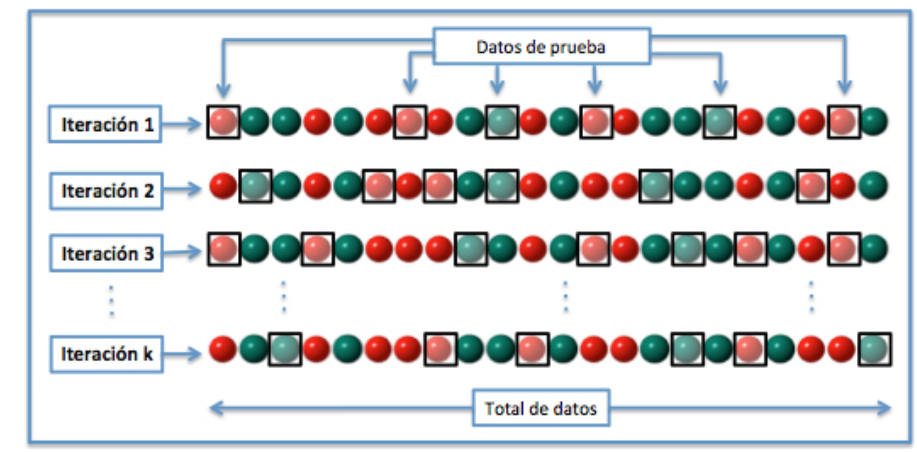

Figura 3.7: Esquema de funcionamiento de la validación cruzada [194]

los valores atípicos son comunes en los conjuntos de datos EEG. Debido a esto, la aplicación de algoritmos de regularización permitirá obtener mejores resultados en las clasificación de señales en entornos BCI.

Años después de que Friedman propusiera su modelo, algunos autores [189] propusieron un método eficiente para seleccionar el parámetro de regularización poniendo de manifiesto el potencial del algoritmo LDA regularizado o RLDA. Este algoritmo aporta una estrategia simple para superar el problema de la singularidad aplicando un término de regularización que es comunmente estimado a través de validación cruzada, a partir de una terna de posibles candidatos. Sin embargo, la validación cruzada puede tener un coste computacional inasumible cuando el conjunto a evaluar es demasiado grande. En dicho trabajo se presenta un algoritmo eficiente para RLDA que computa la transformación óptima de RLDA para un ámplio número de parámetros candidatos, todo ello teniendo prácticamente el mismo coste que ejecutando el algoritmo RLDA un número pequeño de veces. De hecho, esto facilita la selección del parámetro de forma eficiente para RLDA. Del análisis realizado en dicho trabajo se desprenden importantes conclusiones que refuerzan el uso de RLDA. Los experimentos muestran que para un parámetro de regularización apropiadamente seleccionado, el funcionamiento de RLDA genera unos resultados mucho más favorables que otros algoritmos basados en LDA e incluso que SVM (Support Vector Machines), cuyas características se abordarán a continuación.

El algoritmo LDA ha sido utilizado en numerosas ocasiones para la clasificación de señales EEG en entornos BCI. Estudios recientes [49] han demostrado las buenas prestaciones del algoritmo LDA, en su implementación clásica de Fisher, para la 
clasificación de señales EEG. La utilización de este algoritmo está asociada al cumplimiento de determinadas condiciones por parte de los datos analizados. Tal y como se ha comentado anteriormente, para obtener una solución única de los parámetros que definen el discriminante debe existir normalidad multivariada e igualdad de matrices de covarianzas entre los grupos de patrones de las dos clases. En este experimento se consiguió una precisión en la clasificación de un $80 \%$. También han reportado buenos resultados en la precisión de clasificación otros experimentos BCI en los que se ha utilizado el algoritmo LDA [43]. Este estudio describe un nuevo tipo de sistema BCI que utiliza un prototipado para habilitar una transición ágil de varios tipos de estimación paramétrica y algoritmos de clasificación a una implementación y prueba en tiempo real. Este sistema es capaz de procesar múltiples canales EEG de forma online y opera bajo sistema operativo Windows en tiempo real en un PC standar, sin ningun tipo de procesador de señal digital. El sistema BCI fue probado con 3 ususarios que fueron requeridos a imaginar movimientos de su mano a izquierda y derecha. Con los condicionantes anteriormente descritos y la aplicación del algoritmo LDA se obtuvo una precisión en la clasificación de entre el $70 \%$ y el $90 \%$ utilizando dos canales EEG. Algunos autores [195] han utilizado el algoritmo LDA como algoritmo de clasificación en el desarrollo de un nuevo modelo de sistema BCI basado en P300. Las mejoras introducidas estan orientadas a disminuir el tiempo y el número de pruebas realizadas durante el entrenamiento respecto de las que se venían realizando hasta ese momento. Todo ello sin sacrificar las prestaciones del sistema y manteniendo un alto rendimiento desde su puesta en marcha.

Sorprendentemente y a pesar de las bondades descritas anteriormente, el algoritmo LDA con regularización ha sido mucho menos utilizado en entornos BCI que el algoritmo LDA en su implementación de Fisher. Algunos ejemplos de aplicación del algoritmo RLDA han demostrado el potencial de este algoritmo para la clasificación de señales EEG que aconsejarían incrementar su utilización en este tipo de entornos. Algunos autores [154] han utilizado potentes argumentos en favor de la utilización del algoritmo LDA regularizado en entornos BCI. Este estudio pone de manifiesto la idea de que no existen evidencias de que los métodos de clasificación lineal arrojen peores resultados que los clasificadores no lineales. Se posiciona por tanto a favor de la utilización de métodos de clasificación lineal no sólo por su simplicidad, sino 
Capítulo 3. Análisis, procesado y clasificación de señales EEG

también por su rendimiento. En segundo lugar, también se tiene en cuenta la regularización, principio altamente relevante en condiciones experimentales que suelen darse durante el trabajo con sistemas BCI, al disponer de un número pequeño de muestras de entrenamiento con características "débiles". Clasificar este tipo de características con un número pequeño de muestras puede llevar a problemas de sobreentrenamiento del clasificador que limiten la capacidad de generalización. Para eliminar esto, los autores proponen elegir entre dos posibilidades: establecer una etapa robusta de pre-procesamiento para extraer vectores de características de pocas dimensiones que puedan ser tratados por la mayoría de clasificadores, o bien, optar por introducir regularización en el algoritmo de clasificación, de tal forma que las características con alta dimensionalidad puedan ser manejadas también con un pequeño número de muestras de entrenamiento. La utilización del algoritmo RLDA de Friedman [188] es la opción preferida por los autores del mencionado estudio, utilizando validación cruzada con $k=10$ para la estimación del error de generalización así como para la selección del parámetro óptimo de regularización.

Otro tipo de clasificadores lineales son las SVM. Este tipo de clasificadores guardan similitudes con los clasificadores de análisis de discriminante lineal debido a que utilizan también un hiperplano discriminante para separar cada una de las clases. Sin embargo, la forma en la que se selecciona dicho hiperplano es la principal diferencia entre estos dos tipos de algoritmos. Una SVM es un sistema de aprendizaje máquina que permite resolver problemas de clasificación y regresión de manera muy eficiente, ofreciendo un mejor rendimiento que otras técnicas (como las redes neuronales). El éxito de las SVM radica en tres puntos fundamentales: poseen una sólida fundamentación matemática, se basan en minimizar la probabilidad de una clasificación erronea sobre nuevos ejemplos y disponen de potentes herramientas y algoritmos para hallar la solución de manera rápida y eficiente [196]. El entrenamiento de los algoritmos SVM es relativamente fácil; en estos algoritmos no existe un óptimo local (tal y como se verá en el caso de las redes neuronales). Se escalan relativamente bien para datos en espacios con alta dimensionalidad y mantienen un compromiso controlado explícitamente entre la complejidad del clasificador y el error. Además, es posible utilizar como entrada datos no tradicionales como cadenas de caracteres al algoritmo SVM. Todas estas fortalezas de este tipo de algoritmos han sido 


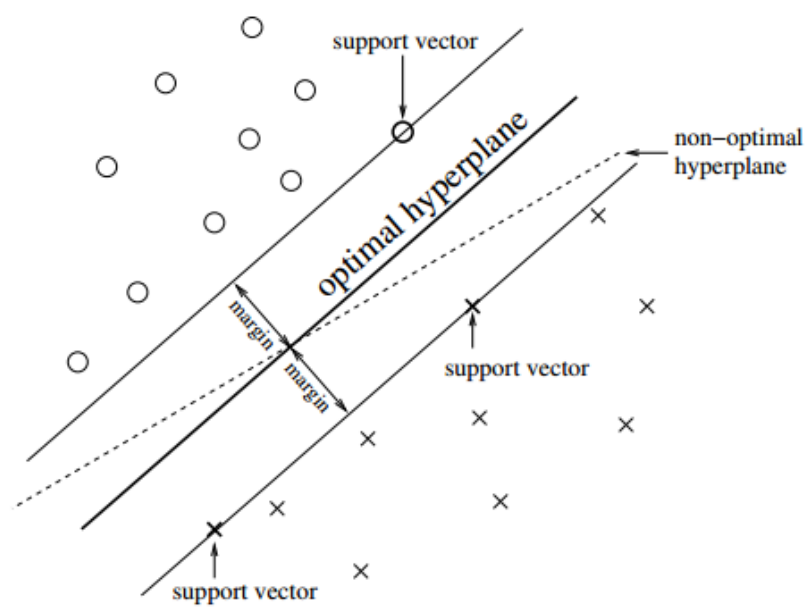

Figura 3.8: Esquema de funcionamiento de los algoritmos SVM ([134])

descritas en la literatura científica [197]. A todo lo anterior hay que añadir que las SVM poseen una buena capacidad de generalización y que guardan algunas similitudes con el algoritmo RLDA. No en vano, las SVM también utilizan un parámetro de regulación "p". La utilización de funciones de base radial o RBF ha permitido crear límites no lineales de decisión mediante este aumento en la complejidad del clasificador mediante el conocido como "truco del núcleo" [198].

Algunos autores [199] [200] [201] han utilizado con éxito el algoritmo SVM en experimentos BCI. Hace algunos años [200] la comunidad científica describía las SVM como herramientas de aprendizaje máquina que han sido ampliamente adoptadas debido a su capacidad para lograr altas capacidades de generalización. Las SVM han sido básicamente diseñadas para problemas de clasificación binaria y emplean aprendizaje supervisado para encontrar el hiperplano óptimo de separación entre dos clases de datos. En dicho trabajo, los autores introducen un algoritmo basado en la correlación cruzada ayudado por un clasificador basado en SVM, utilizando la correlación cruzada para la extracción de características. La técnica propuesta ha sido utilizada para la clasificación binaria de señales EEG utilizando las características adecuadas, extraídas de las correlaciones cruzadas de la señal EEG. La utilización de este método ha demostrado un aumento de la precisión en la clasificación de la señal EEG, pasando de un $94.5 \%$ con un método propuesto recientemente a un $95.96 \%$ de precisión en la clasificación obtenida con el nuevo metodo. Otros autores [201] han 
Capítulo 3. Análisis, procesado y clasificación de señales EEG

propuesto algoritmos SVM multiclase con códigos correctores de error para problemas de clasificación de señales EEG multiclase. Al igual que en el caso anterior, este experimento también demostró que con una adecuada selección de características, la utilización de las SVM puede asegurar la obtención de altas precisiones en el proceso de clasificación.

Algunos autores [202] han comparado el rendimiento de SVM con otros clasificadores durante la caracterización de un experimento BCI para la clasificación de la señal EEG en cuatro clases, basadas en imaginería motora. La señal EEG fue obtenida con 60 electrodos de cinco sujetos que ejecutaban cuatro tareas diferentes de imaginería motora. Los resultados en la precisión de la clasificación indicaron que las SVM era el método de clasificación que maximizaba la precisión del sistema BCI, mientras que el algoritmo K-NN (que será expuesto en secciones siguientes) obtuvo el peor resultado. Otros autores [160] han intentado comparar el rendimiento del algoritmo LDA con las SVM en su forma no lineal analizando los resultados obtenidos a partir de la clasificación de la señal EEG espontánea durante cinco tareas mentales. El resultado obtenido no fue concluyente, ya que solo arrojó resultados ligeramente mejores para el caso en el que se utilizaban las SVM respecto de los resultados en términos de precisión en la clasificación de las señales obtenidos por el algoritmo LDA.

\section{Redes neuronales}

Las redes neuronales han sido utilizadas en un gran número de experimentos y aplicaciones en entornos BCI [203] [204] [205]. Junto con los clasificadores lineales, son los algoritmos de clasificación más utilizados para clasificar la señal EEG. Segun la literatura científica [206] una red neuronal artificial puede definirse como un procesador distribuido en paralelo de forma masiva que tiene una tendencia natural para almacenar conocimiento de forma experimental y lo hace disponible para su uso. Las redes neuronales constituyen nuevas formas de computación inspiradas en modelos biológicos. Estas estructuras guardan ciertas similitudes con el cerebro. Por un lado, el conocimiento de la red se obtiene mediante un proceso de aprendizaje. Por otro lado los pesos sinápticos existentes entre las distintas neuronas son los que almacenan la información de forma distribuida tras el proceso de aprendizaje. 
Las redes neuronales artificiales permiten resolver problemas de clasificación de alta complejidad debido a su capacidad para separar regiones no lineales de decisión tan complicadas como se desee. La complejidad del sistema dependerá del número de neuronas y de capas.

Las redes neuronales más utilizadas son aquellas con múltiples capas, compuestas por un conjunto de nodos de entrada que componen la capa de entrada, un conjunto de una o más capas ocultas de neuronas y una capa de neuronas de salida. En la literatura científica es posible encontrar estudios [207] en los que se enumeran los distintos tipos de redes neuronales utilizadas en procesos de reconocimiento de patrones o aprendizaje máquina. El perceptrón multicapa (MLP, Multi Layer Perceptron) es el modelo de red neuronal artificial más utilizado en la práctica, tanto para la resolución de problemas de clasificación como en problemas de regresión [208]. El modelo de red surgió en los años 80 debido a las limitaciones presentadas por el perceptrón simple, ya que no existia posibilidad de aprender clases de funciones no linealmente separables [209]. La literatura especializada [208] describe en detalle la evolución desde el perceptrón simple hasta el perceptrón multicapa, a través de la inclusión de una o varias capas ocultas siguiendo una estructura similar a la descrita en la Figura 3.9. En este sentido, el perceptrón multicapa puede aproximar cualquier función continua siempre que contenga al menos una capa oculta. Por tanto, asegurando un número suficiente de neuronas en la capa o capas ocultas, las redes MLP pueden funcionar como aproximadores de funciones universales. El problema se centrará, en función del conjunto a clasificar, en el número de neuronas ocultas necesarias para conseguir un rendimiento óptimo. Cuando la literatura científica habla de perceptrón se esta refiriendo a una estructura de perceptrón multicapa que no posee capas ocultas. La Figura 3.10 desribe las distintas configuraciones del MLP junto con las fronteras de decisión a las que pueden dar lugar. Algunos autores han comparado el rendimiento del MLP con la aplicación directa del algoritmo LDA a los datos de entrada [210]. La comparación entre ambos algoritmos es posible debido a que el algoritmo LDA es equivalente al MLP cuando este no posee capas ocultas.

El uso de perceptrones multicapa presenta importantes ventajas pero también algún inconveniente. Debido a la capacidad de los perceptrones multicapa para ser aproximadores universales, esto también los hace sensibles al exceso de entrenamien- 


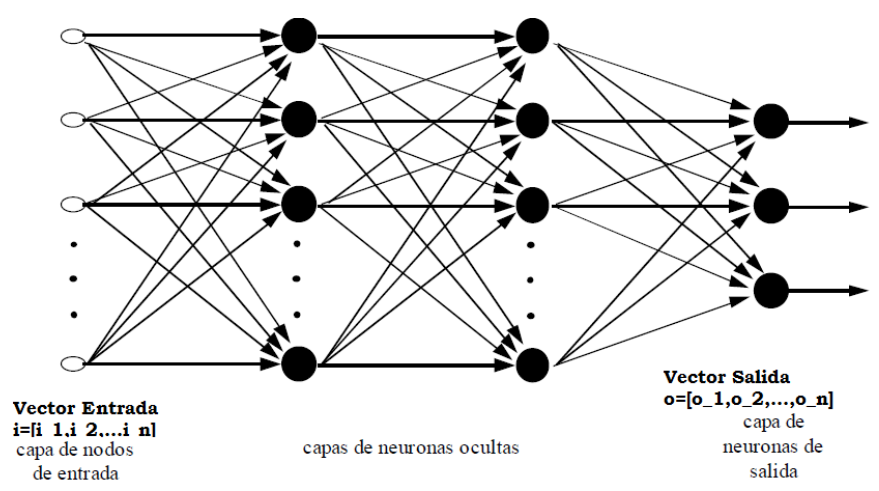

Figura 3.9: Estructura de un perceptrón multinivel [206]

to bajo condiciones similares a las que presentan los datos utilizados en experimentos BCI. Algunos autores [205] han puesto de manifiesto en sus investigaciones este problema, que surge con la introducción del MLP como algoritmo en entornos BCI. Dicho estudio propone introducir un algoritmo evolutivo con el objetivo de entrenar un sistema BCI basado en un MLP. A la vista de los resultados obtenidos, la introducción de este tipo de entrenamiento consigue evitar el sobreajuste. Los resultados experimentales muestran un MLP con una capacidad de generalización mucho mayor que otros experimentos de la literatura científica. El experimento consistía en entrenar un MLP para clasificar un conjunto de entrenamiento contenido en una matriz de 268x5376 en dos clases. El conjunto de test fue utilizado después del proceso de entrenamiento para verificar la calidad del clasificador MLP obtenido. El algoritmo evolutivo, junto con el MLP, permite evitar el sobreajuste debido a la no utilización de un algoritmo de búsqueda local. Se podría concluir que el tamaño del MLP, la velocidad de entrenamiento y la calidad de la clasificación son tres parámetros clave que se encuentran en conflicto al utilizar MLP como base para sistemas BCI.

Hace una década, y basándose en experiencias previas, algunos autores [211] ya apuntaban los resultados prometedores que la aplicación de redes neuronales podría generar en el campo de la clasificación de señales EEG. El proceso de toma de decisión se realizó en dos etapas: inicialmente se utilizó la transformada wavelet (WT) para la extracción de características para posteriormente utilizar algoritmos neuronales para la clasificación. Este estudio muestra un ejemplo en el que se combinan dos tipos de algoritmos de clasificación para utilizar las mejores características de cada uno de 


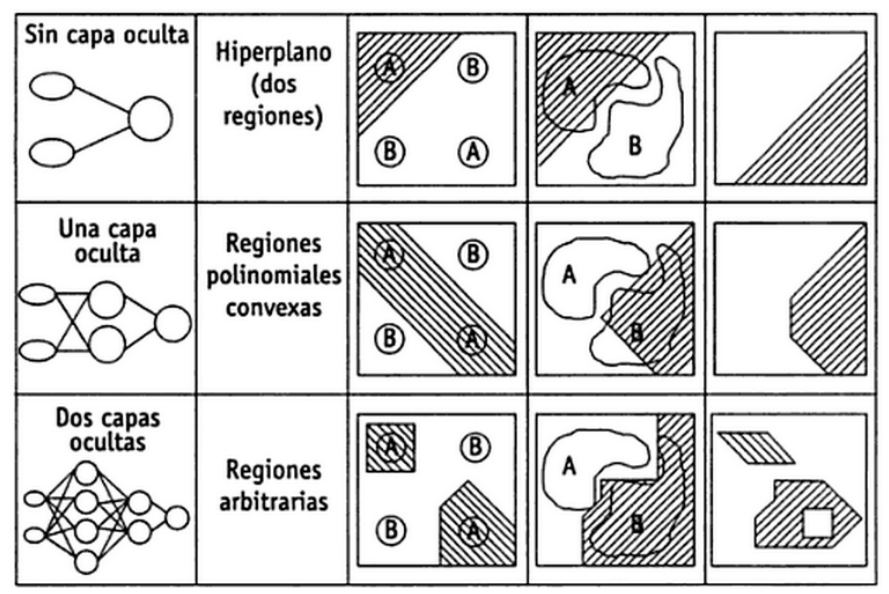

Figura 3.10: Regiones de decisión en función de la configuración del perceptrón [208]

ellos en función de las necesidades del conjunto analizado. Por un lado se utiliza el MLP, con un esquema de gradiente descendente durante el proceso de clasificación. Por otro lado se utiliza una red RBF. Este tipo de redes neuronales compuestas por funciones de base radial se entrenan de forma mucho mas rápida que los MLP y no muestran ninguno de los problemas de entrenamiento que sí muestra el MLP tales como problemas con el mínimo local o parálisis. En las redes RBF la activación de la neurona oculta se determina en dos etapas: En primer lugar se computa la distancia euclidea entre el vector de entrada y el centro $c_{i}$ que representa la iésima neurona oculta. En segundo lugar se aplica una función $h$, normalmente gausiana, y se utiliza la distancia obtenida para obtener la activación final de la neurona oculta. Tal y como ocurre en este experimento, la función suele ser gausiana:

$$
G(x)=\exp \left(-\frac{x^{2}}{\sigma^{2}}\right)
$$

El esquema de clasificación múltiple utiliza 16 subsistemas de clasificación. Para cada uno de estos subsistemas, se utilizará una estructura MLP o RBF. El análisis de la señal EEG consistió en examinar la diferencia entre las señales EEG normales y aquellas relacionadas con un proceso epiléptico. La información estadística extraída tras la aplicación de la transformada wavelet se utilizó como entrada a un conjunto de algoritmos de aprendizaje supervisado compuesto por redes RBF y MLP. Ambos esquemas de clasificación fueron capaces de obtener buenos resultados en la clasifi- 
Capítulo 3. Análisis, procesado y clasificación de señales EEG

cación.Tras la utilización de MLP, la precisión de clasificación para el problema de dos clases fue del $97 \%$. Para el caso de RFB, se obtuvo una precisión del $98 \%$ con un periodo de entrenamiento sustancialmente menor que el utilizado por MLP.

Hace más de una década, se propuso un nuevo clasificador neuronal local [212] para el reconocimiento de tareas mentales de forma online analizando las señales EEG espontáneas. El clasificador estába incluido en un sistema BCI portátil llamado ABI, que había sido evaluado con cuatro sujetos sanos. Se analizó el funcionamiento offline con estos sujetos y para tres de ellos también de forma online, incluyendo retroalimentación. El sistema BCI portátil propuesto reconoce tres tareas mentales diferentes a partir de señales EEG espontáneas de forma online. La precisión del sistema está en torno al $70 \%$. Este resultado modesto es ampliamente compensado por dos propiedades del sistema ABI: Por un lado las respuestas erróneas estaban por debajo del 5\%, y por otro lado el sistema ABI toma una decisión cada medio segundo. Además, debido a que el sujeto y su sistema personal ABI aprenden de forma simultánea uno del otro, el sujeto es capaz de controlar totalmente el sistema en poco tiempo. De hecho, los resultados muestran cómo uno de los sujetos fue capaz de conseguir un control excelente en sólo 5 dias de entrenamiento.

Un clasificador gaussiano utiliza la distribución de probabilidad de las muestras disponibles para determinar si una muestra concreta pertenece o no a una clase. En primer lugar se obtienen los centros de masa de las distintas clases del problema de clasificación, y posteriormente se calcula la probabilidad entre la muestra a clasificar y dichos centros obtenidos. Por tanto, se obtiene la distancia entre la muestra y la dispersión de probabilidad de cada clase. Algunos autores han realizado experimentos de clasificación de señales EEG con un conjunto de varias unidades gausianas [213]. Cada una de las unidades gausianas se corresponde con cada una de las tareas mentales o clases que debían ser reconocidas. Por tanto, conseguir posicionar adecuadamente las gausianas en el espacio y asignarles una varianza apropiada son dos aspectos clave para asegurar un funcionamiento óptimo del sistema. Los clasificadores fueron entrenados de forma offline con un conjunto de una sesión de entrenamiento dada. La primera sesión de entrenamiento fue utilizada para agrupar las señales iniciales EEG para entrenar el clasificador y por tanto los usuarios no recibían ningún tipo de retroalimentación en este caso. Una mejora clara en el funcionamiento del 
clasificador pudo ser observada en términos de errores y respuestas "desconocidas". El clasificador etiquetaba las muestras en base a cuatro clases predefinidas: 1, 2, 3 o "desconocido". Cada una de las clases se corresponde con transiciones entre comportamientos determinados por los estados mentales del usuario. La salida del sistema BCI queda determinada por las transiciones generadas en una máquina de estados. El conjunto de test se evaluó de forma online en la siguiente sesión.

\section{Clasificadores Bayesianos}

La clasificación supervisada es una parte importante del aprendizaje máquina con un gran número de aplicaciones en diversos campos como el reconocimiento de patrones, el diagnóstico medico o el reconocimiento de imágenes [214] [215]. De forma general, la clasificación supervisada asume la exisencia de dos tipos de variables. Por un lado se encuentran las variables predictoras $X=\left(X_{1}, X_{2}, \ldots, X_{n}\right)$ y por otro lado la variable de clase $C$ que toma valores dentro de un rango definido $\left(1,2,3, \ldots C_{n}\right)$. El objetivo principal de los clasificadores supervisados es tratar de aprender las relaciones entre las variables predictorias y una clase determinada, de tal forma que sea posible asignar uno de los valores de $\mathrm{C}$ a un nuevo conjunto de variables no observadas $x=\left(x_{1}, x_{2}, \ldots, x_{n}\right)$ en el que el valor de la clase es desconocido [216]. Uno de los métodos utilizados en numerosas ocasiones para trabajos de clasificación supervisada son las redes bayesianas [217] [218]. Estas redes permiten modelar la distribución de probabilidad de un conjunto de datos disponible. Las redes bayesianas se consideran métodos de clasificación generativos debido a que el objetivo principal durante el aprendizaje es maximizar la verosimilitud conjunta mediante la búsqueda de aquel modelo que represente mejor al conjunto de datos de partida. También puede darse el caso de que se busque maximizar la verosimilitud condiconal, en cuyo caso se estará ante un aprendizaje discriminativo. La diferencia principal entre el aprendizaje discriminativo y el generativo se basa en el hecho de que el aprendizaje discriminativo es una aproximación más natural al aprendizaje de modelos de clasificación debido a que está fundamentado en la probabilidad condicional $p\left(C \mid X_{1}, X_{2}, \ldots, X_{n}\right)$. Este valor de probabilidad es el que se utiliza para clasificar nuevas muestras en las que el valor de la variable de clase es desconocido, una vez que se ha aprendido el modelo. Por otro lado, se ha de tener en cuenta que el cálculo de la verosimilitud conjunta puede 
ser obtenido mediante una fórmula cerrada, haciendo posible un cómputo eficiente. Sin embargo, en el cálculo de la verosimilitud condicional esto no es posible, siendo el aprendizaje discriminativo de modelos de clasificación bayesianos mucho más costoso computacionalmente que la aproximación generativa análoga [216].

El clasificador bayesiano más simple es el modelo naive Bayes [219]. Un marco ampliamente utilizado para propósitos de clasificación basado en la fórmula de bayes o el teorema de bayes

$$
P\left(C=c_{k} \mid X=x\right)=P\left(C=c_{k}\right) \frac{P\left(X=x \mid C=c_{k}\right)}{P(X=x}
$$

donde $P\left(C=c_{k} \mid X=x\right)$ es la probabilidad condicional de que una muestra pertenezca a la clase $c_{k}$, asumiendo que conocemos que tiene un fector de características $x$. La regla de Bayes, especifica cómo esta probabilidad condicional puede ser computada a partir de las probabilidades condicionales de observar valores de características particulares para muestras de cada clase, y la probabilidad incondicional de observar un documento de cada clase.

El clasificador naive Bayes simplifica de forma importante el proceso de aprendizaje asumiendo que las variables de predicción son independientes de las clases dadas. Aunque la independencia es generalmente una asunción pobre, en la práctica el clasificador naive Bayes puede compararse de forma fiable con otros clasificadores más sofisticados. Algunos autores [219] han estudiado de forma empírica el funcionamiento del clasificador naive Bayes tratando de comprender aquellos aspectos que afectan al rendimiento de este clasificador. Las limitaciones son bien conocidas: En el caso de características binarias, este algoritmo solo puede aprender funciones discriminantes lineales. Este hecho hace que nunca se obtenga el punto óptimo en casos no separables linealmente. En otro caso, para un cierto número de características, naive Bayes es capaz de aprender funciones polinómicas de discriminación. Así, la separaibilidad polinomial es una condición necesaria, pero no suficiente, para conseguir un óptimo mediante naive bayes cuando se trata de características en un dominio finito. El estudio citado demuestra que naive Bayes tiene un buen funcionamiento para situaciones en las que las características tienen una cierta dependencia. Se concluye que el clasificador obtiene su mejor funcionamiento bajo dos escenarios opuestos: por 
un lado en aquellos casos en los que existen variables completamente independientes y por otro lado aquellos casos en los que las características son funcionalmente dependientes. Algunos autores [216] argumentan que el modelo naive Bayes tiene una estructura fija que no depende de los datos, por tanto no es posible decir que el aprendizaje estructural de este modelo siga un criterio generativo o discriminativo. Sin embargo el aprendizaje habitualmente utilizado para obtener los parámetros del modelo naive Bayes, que coincide con el procedimiento general de obtención de parámetros en los clasificadores Bayesianos, está basado en las estimaciones máxima verosimilitud (ML) o máximo a posteriori (MAP) del conjunto de parámetros. Estas aproximaciones pueden considerarse generativas al aprendizaje de los parámetros del clasificador, ya que utilizándolas se pretende maximizar la verosimilitud de los datos dado el modelo.

Hace algunos años se trató de optimizar el análisis de la señal EEG mediante la mejora de los clasificadores y del funcionamiento de los sistemas BCI [220]. Los autores trataron de introducir un nuevo modelo de exploración de la señal EEG basada en el análisis estadístico de la transformada wavelet continua (CWT) y la "wavelet coherence" (WC). Los resultados mostraban que la metodología propuesta era capaz de identificar regiones en el espectro tiempo/frecuencia durante una serie de tareas específicas BCI realizadas. Además, se identifica un ejemplo de región y los patrones son clasificados utilizando el clasificador Naive Bayes. Según los autores, las características innovadoras del proceso justifican la posibilidad del modelo propuesto para aplicarlo a otro tipo de escenarios. Otros experimentos [173] han comparado la precisión de un sistema BCI que utiliza a usuarios entrenados para desplazar un cursor en una o dos dimensiones a través de señales EEG. Se ha realizado una doble comparación con el objetivo de conseguir la máxima precisión en la decodificación. En primer lugar, se ha analizado el número óptimo de características a utilizar para maximizar la precisión total del sistema. En segundo lugar, se han comparado tres tipos de clasificadores basados por un lado en el análisis del discriminante lineal y en el teorema de bayes por otro. A pesar de que el clasificador basado en el teorema de Bayes no obtuvo el mejor resultado en términos de precisión, estuvo muy cerca de los resultados arrojados por el mejor de los clasificadores que utilizaron funciones de discriminante lineal para realizar la decisión. Otros experimentos realizados 
Capítulo 3. Análisis, procesado y clasificación de señales EEG

en el pasado [221] han propuesto métodos para BCI basados en modelos gráficos probabilísticos que superan algunas de las limitaciones de los enfoques basados en clasificadores de señales. Los autores modelaron la dinámica del cerebro a nivel de las relaciones amplitud-frecuencia ocultas, utilizando una red bayesiana dinámica que se aprende a partir de datos obtenidos de la señal EEG. En este sentido, se demostró que el modelo puede utilizarse para inferir las distribuciones de probabilidad sobre variables de estado ocultas, las cuales corresponden a funciones cerebrales, útiles para establecer un modelo de interface. El uso del modelo proporcionó una estimación probabilística continua del estado del cerebro, permitiendo modelar la estructura causal y las dependencias entre los diferentes estados cerebrales.

También se han utilizado clasificadores bayesianos para la clasificación de señales EEG P300 [222]. Los autores utilizan estímulos visuales consistentes en ocho flechas aleatoriamente intensificadas para la selección del target correspondiente a una dirección. La clasificación está basada en enfoque bayesiano y se utiliza el conocimiento estadístico a priori de las componentes de los targets. Los eventos son entonces agrupados para mejorar su detección. El sistema tiene un funcionamiento adaptativo que consigue amoldarse al usuario y a la calidad de la onda P300. Los algoritmos de clasificación fueron desarrollados de forma offline para el proceso de entrenamiento y posteriormente validados tanto offline como online. El sistema obtuvo una tasa de transferencia de 7 comandos/minuto con $95 \%$ de falsos positivos en la precisión de la clasificación.

Como alternativa al uso de clasificadores lineales, existe la posibilidad de utilizar clasificadores bayesianos no lineales, basados en el modelo generativo, que producen fronteras no lineales de decisión. Esto genera unas ventajas evidentes debido a que aumenta el rendimiento del clasificador y disminuye su coste computacional. Sin embargo, los clasificadores bayesianos no lineales no han sido generalmente utilizados en aplicaciones BCI.

\section{Cadenas Ocultas de Markov}

Los modelos ocultos de Markov fueron descritos en la decada de 1960 por LE Baum [223]. Un modelo oculto de Markov (HMM o Hidden Markov Model) desarrolla un modelo estadístico basado en un sistema cuyos parámetros deben ser mode- 


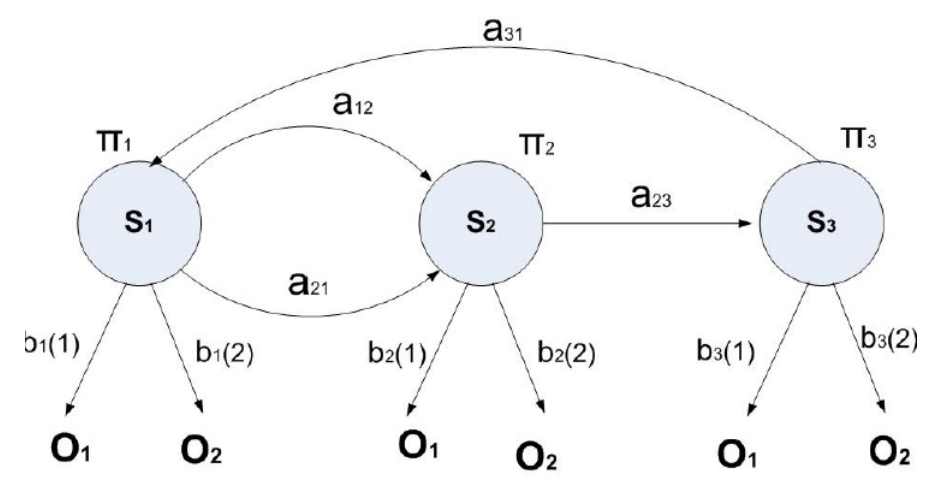

Figura 3.11: Representación grafica de un HMM ([225])

lados. La determinación de los parámetros ocultos de la cadena de markov en base a una serie de observaciones constituye el objetivo principal de este método. Un HMM se puede considerar como la red bayesiana dinámica más simple. Se establece una representación matemática del problema que permite explicarlo, al tiempo que permite analizar el problema sin que sea necesario observarlo de forma directa. Las HMM deterministas analizan características conocidas de la señal, mientras que los estadísticos caracterizan la señal con valores estadísticos. Los modelos ocultos de markov parten de las cadenas de Markov [224] en las que las observaciones están representadas como un proceso aleatorio paramétrico. En dicho proceso, es posible determinar el valor de los parámetros con una precisión suficiente. La Propiedad markoviana [225] establece que la probabilidad condicional de cualquier suceso en cualquier instante "futuro" dada la ocurrencia de cualquier suceso "pasado" y el estado actual es independiente de lo acontecido en el pasado y más bien solo depende del estado acutal del sistema analizado. En un Diagrama de Estados, cada nodo representa a un elemento del espacio muestral (Estado) y cada arco dirigido a la probabilidad de transición Pij (desde i a j) asociada al par de estados que conecta (i , j). Las Cadenas de Markov Ocultas son modelos en los que se rastrean y encuentran algunos estados que eran ocultos para las Cadena de Markov simples. Esto hace que el modelo oculto tenga una gran eficiencia en la estimación de sus probabilidades y un gran alcance. Las HMM poseen un modelo doblemente estocástico en el cual hay un proceso subyacente que está oculto y que sólo puede ser detectado a través de las observaciones. En la Figura 3.11 se describe gráficamente un HMM. 
Los modelos ocultos de Markov han sido especialmente aplicados en casos de reconocimiento del habla [226], de escritura manual [227] o de gestos [228]. Sin embargo, también se han aplicado al análisis de la señal EEG espontánea para controlar un teclado virtual [229]. En este caso, la actividad EEG de tres sujetos es modulada a través de movimientos imaginarios de la mano y la pierna para operar el teclado virtual. Los resultados obtenidos por los tres sujetos muestran que el diseño propuesto de teclado virtual (VK) es útil como alternativa a los dispositivos de deletreo. Los sujetos podrían conseguir una tasa de deletreo que varía entre 1.02 y 0.67 letras por minuto. Algunos autores [230] han contribuido a desarrollar el uso de los modelos markovianos en general, y los HMM en particular, para distinguir entre tres tareas mentales cognitivo-motoras en un sistema BCI basado en un protocolo asíncrono. En este protocolo, el sujeto no sigue ningún tipo de esquema fijo, sino que está instruido para concentrarse de forma repetitiva en una tarea mental para una cantidad aleatoria de tiempo y cambia directamente al siguiente, sin pasar por el estado de reposo. Asi, la señal asociada con cada tarea mental representa una secuencia continua de eventos mentales sin tener un principio o un final definido. A partir de esto, los modelos markovianos deben extraer información para la clasificación. En dicho experimento, los potenciales EEG fueron obtenidos a través de un sistema portatil, utilizando 32 electrodos localizados en una posición estandar del sistema internacional 10-20. De los resultados obtenidos en este experimento deben señalarse dos aspectos importantes sobre el modelo markoviano de la señal EEG: En primer lugar, la superioridad de modelos discriminantes como IOHMM (Input Output Hidden Markov Models [231]) sobre los modelos generativos como HMM. En segundo lugar, la falta de ventajas práticas en el uso de modelos secuenciales cuando la información a priori no puede ser utilizada para construir una estructura adecuada, como en el caso del modelado de los ritmos EEG con un sistema BCI asíncrono.

Otros experimentos [232] han utilizado HMM para la clasificación online de datos EEG durante la imaginación de movimientos de la mano a derecha e izquierda. Los resultados tras el proceso de clasificación muestran una mejora del experimento online en comparación con los métodos de clasificación lineal. Los autores pretenden introducir un nuevo sistema BCI basado en un clasificador HMM para los patrones EEG obtenidos durante la imaginación de movimientos a derecha e izquierda. 


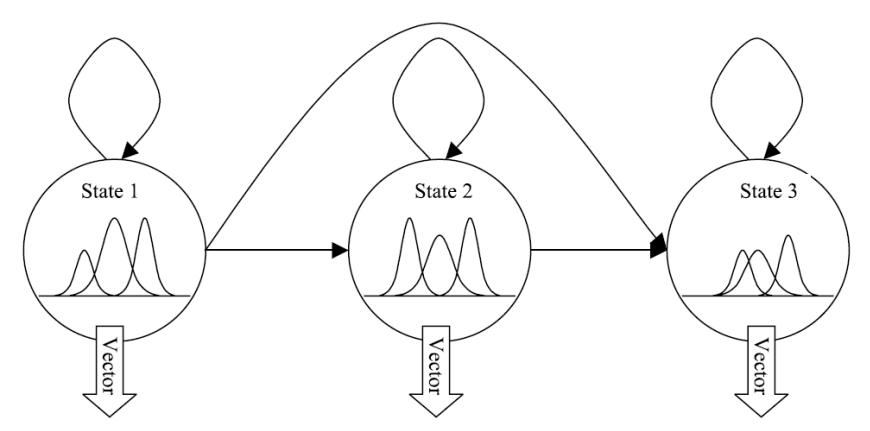

Figura 3.12: HMM utilizado en el sistema BCI-HMM [232] con $s=3$ estados. En la figura, las flechas indican las transiciones permitidas, un vector de características $m=3$ es emitido en cada punto de tiempo. El HMM ha sido diseñado como un modelo de izquierda a derecha, debido a que las transiciones son permitidas desde un estado a si mismo y a cualquier estado vecino de la derecha

Además, se ha efectuado una comparación entre los sistemas BCI basados en un algoritmo lineal y en HMM. Las HMM pueden ser vistas en si mismas como un autómata finito, que contiene $s$ estados discretos, emitiendo un vector de características en cada punto de tiempo que depende del estado actual (Figura 3.12). Cada vector de características es modelado utilizando $m$ gausianas por estado. Las probabilidades de transición entre estados son descritas utilizando una matriz de transición. Los autores concluyen que los sistemas BCI basados en HMM pueden ser utilizados para la clasificación online de patrones espacio-temporales EEG durante la imaginación motora.

\section{Vecino más cercano o K-NN}

Por último, dentro de los algoritmos de clasificación para sistemas BCI más utilizados se encuentran los algoritmos de vecino más cercano o "K-NN". Son algoritmos con reglas muy simples y destacan por su sencillez. Se basan en la asignación de un vector de características a una clase, de acuerdo con su vecino más cercano. Estos métodos fueron desarollados en 1951 y han sido objeto de mejoras y comentarios a lo largo de los últimos años [233].

El método de clasificación K-NN realiza un aprendizaje inductivo supervisado. El aprendizaje es inductivo debido a que se obtienen conclusiones generales de información específica y se crean modelos de conceptos a partir de la generalización 
de ejemplos simples, buscando patrones comunes que expliquen los ejemplos [179]. El método K-NN es un metodo de clasificación no paramétrico que estima el valor de la función de densidad de probabilidad, o directamente la probabilidad a posteriori de que un elemento $x$ pertenezca a una clase $C_{j}$ partiendo de la información suministrada por el conjunto de muestras disponibles. En este sentido, las reglas de clasificación por vecindad basan su búsqueda en un conjunto de prototipos de los k prototipos más cercanos al patrón a clasificar. En este tipo de algoritmos, se debe especificar una métrica concreta con el objetivo de evaluar la proximidad. Suele utilizarse por razones computacionales la distancia euclídea $\delta\left(x_{i}, x_{j}\right)$ [234]. A partir de esta distancia, se define un conjunto de referencia que aglutina a una serie de muestras sobre las que se buscarán los vecinos más cercanos. El espacio se divide en regiones por localizaciones y etiquetas de los ejemplos de entrenamiento. Un punto en el espacio es asignado a la clase $C_{i}$ si esta es la clase más frecuente de entre los k ejemplos de entrenamiento más cercanos, habiendo definido previamente los límites del espacio observable.

$$
\delta\left(x_{i}, x_{j}\right)=\sqrt{\sum_{r=1}^{p}\left(x_{i r}-x_{j r}\right)^{2}}
$$

Dentro del método K-NN, la regla de vecindad más simple es la regla de clasificación del vecino más cercano 1-NN. Esta regla de vecindad está basada en asumir que la clase de la muestra que se desea etiquetar es la de la muestra más cercana en el conjunto de referencia definido. Durante el proceso de clasificación, el vecino del elemento a clasificar puede ser un vector de características del conjunto de entrenamiento (como en el caso de vecinos más cercanos (K-NN)), o una clase prototipo (como en la distancia de Mahalanobis).

Algunos autores [236] han abordado uno de los principales problemas del algoritmo K-NN, que no es otro que su sensibilidad al ruido. Los autores procedieron a la eliminación de instancias ruidosas para mejorar la calidad de la clasificación de los $\mathrm{k}$ vecinos más cercanos. Concretamente, se basaron en un método existente de selección de instancias basada en conjuntos rugosos difusos. Otra de las deventajas del algoritmo que los autores dejaron patente es la de su bajo rendimiento ante problemas con alta dimensionalidad. También ha de tenerse en cuenta que K-NN necesita mucho almacenamiento debido a que el conjunto de entrenamiento com- 


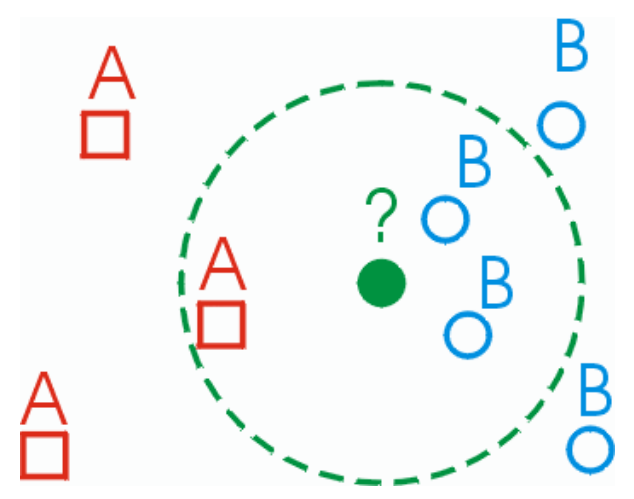

Figura 3.13: Ejemplo de clasificación de la muestra '?'mediante K-NN en un espacio muestral de dos clases ([235])

pleto tiene que ser almacenado para la clasificación de nuevas instancias. El coste de computación también es relativamente alto, debido a que es necesario calcular las coincidencias entre la instancia nueva y todas las instancias de entrenamiento. Este método supone que, evaluando sólo a los vecinos cercanos, es posible obtener la mayor precisión en la clasificación y esto se hace utilizando todos los atributos. Sin embargo, se genera un inconveniente, ya que es posible que se tengan muchos atributos irrelevantes que dominen sobre la clasificación y unos pocos atributos relevantes perderían peso entre otros muchos que fuesen irrelevantes. Por último, cabe destacar la gran susceptibilidad a los datos ruidosos, ya que considera todos los datos igual de relevantes, no teniendo en cuenta que el conjunto de entrenamiento pueda contener datos incorrectos. Estas desventajas en el uso de K-NN hacen que su uso en sistemas BCI para la clasificación de señales EEG esté circunscrito a unos pocos casos. En uno de esos casos [202] se estudió la precisión que podría obtenerse mediante la utilización de K-NN basado en la teoria Dempster-Shafer. Para extraer las características de la señal EEG se utilizaron modelos autorregresivos y descomposición "wavelet". Para evaluar la precisión del metodo de clasificación se utilizó un conjunto de señales EEG obtenidas durante la ejecución de cinco tareas mentales diferentes. Los autores mostraron que el clasificador K-NN basado en Demptster-shafer obtuvo una mayor tasa de precisión durante el proceso de clasificación comparando con los resultados tanto con el K-NN de votación clásico como con el K-NN de distancia-pesos.

La comparación entre los distintos métodos disponibles para la clasificación de señales EEG en entornos BCI ha sido objeto de estudio en el pasado [202]. Los autores 
Capítulo 3. Análisis, procesado y clasificación de señales EEG

desarrollaron este estudio con el objetivo de determinar y comparar el rendimiento de diferentes métodos de clasificación aplicados a un problema de cuatro clases mediante la extracción de datos EEG. Los datos EEG fueron obtenidos mediante 60 electrodos a partir de cinco sujetos ejecutando cuatro tareas diferentes de imaginería motora. La señal EEG fue modelada mediante un proceso adaptativo autoregresivo (AAR). Los clasificadores utilizados fueron cuatro: análisis de la distancia mínima (MDA), análisis del discriminante lineal (LDA), k-NN, y máquinas de soporte vectorial (SVM). El funcionamiento de los cuatro clasificadores fue cuantificado y evaluado por el coeficiente de cohen kappa. Los resultados arrojaron como conclusión que el algoritmo que mejores resultados obtenía era SVM, siendo el algoritmo K-NN el que peor resultado mostraba tras el proceso de clasificación.

\subsubsection{Problemas en la clasificación en entornos BCI}

La naturaleza de la señal EEG es el origen de determinados problemas durante la clasificación de las muestras recogidas en un experimento BCI. Los clasificadores se enfrentan con problemas generados por la presencia de valores atípicos, posible sobreentrenamiento, número de muestras demasiado bajo, etc. Debido a las peculiaridades de los experimentos BCI, uno de los problemas frecuentes a los que debe enfrentarse un algoritmo de clasificación en este contexto es la llamada "maldición de la dimensionalidad". Este problema aparece en la mayoría de experimentos BCI analizados

en la literatura cientifica [237] [238] [239]. Si el número de muestras disponibles es demasiado pequeño en comparación con el número de dimensiones o vectores de características, el clasificador tendrá dificultades para arrojar buenos resultados. Para la definición de las diferentes clases en las que se deben clasificar las muestras de un experimento BCI, será necesario disponer de un número mínimo de muestras. El número mínimo de muestras necesarias para evitar la maldición de la dimensionalidad, aumenta de forma directa y exponencial con las dimensiones de los vectores de características. En este contexto, las técnicas de selección de características descritas constituyen una pieza clave para tratar la maldición de la dimensionalidad. La selección de características determina un subconjunto de características extraído del conjunto total. Esta selección puede ayudar además a descartar aquellas características que no aportan información útil al proceso de decisión, o aquellas que sólo 
introducen ruido en el proceso, en favor de aquellas que contienen información útil. De esta forma, la reducción del espacio de características se realiza de forma óptima, mejorando el rendimiento del proceso de clasificación [240]. Existen diversos métodos de selección de características que han sido comentados en apartados anteriores [241] [162]. Al margen de la selección de características, es posible utilizar algoritmos con buena capacidad de generalización e inmunidad a la maldición de la dimensionalidad como el SVM (Descrito en sección 3.4).

Otro de los problemas de los procesos de clasificación en entornos BCI señalados por la literatura cientifica [134] es el compromiso existente entre la varianza y el sesgo. En este caso, la varianza representa la sensibilidad del clasificador al conjunto de datos que se usa para entrenarlo y el sesgo representa la divergencia entre la salida estimada y la mejor salida. Para conseguir el menor error en la clasificación, se deben tener valores de sesgo y varianza bajos. Los clasificadores estables tienden a tener un alto sesgo y una baja varianza, mientras que los clasificadores inestables tienen un sesgo bajo y una alta varianza. Esto puede explicar el hecho de que los clasificadores simples superan en ocasiones el rendimiento de otros más complejos. Reducir al mismo tiempo el sesgo y la varianza es el reto para conseguir un menor error durante el proceso de clasificación. En este escenario, el error medio de clasificación depende del ruido, el sesgo y la varianza (ver ecuación 3.4). En dicha expresión, el caracter aleatorio de las muestras que forman tanto el conjunto de test como de entrenamiento está representado por el factor de ruido. La divergencia entre el modelo estimado y el mejor modelo está representado por el sesgo. Por último, el termino de la varianza está directamente relacionada con la sensibilidad del clasificador al conjunto de muestras de entrenamiento empleado.

$$
\text { Error }- \text { Medio }- \text { Clasificacion }=\text { Ruido }^{2}+\operatorname{Sesgo}(f(x))^{2}+\operatorname{Var}(f(x))
$$

Otro de los problemas que afectan a los clasificadores utilizados en entornos BCI es el sobreentrenamiento, al que ya se ha hecho referencia en secciones anteriores. El sobreentrenamiento de un clasificador se genera cuando sólo es capaz de clasificar correctamente las muestras que han sido utilizadas como conjunto de entrenamiento. Esto hace que el clasificador sea incapaz de arrojar buenos resultados cuando 
trata de clasificar cualquier conjunto que difiera sensiblemente del conjunto de entrenamiento. Existen métodos de regularización que pueden limitar los efectos del sobreentrenamiento y limitar el efecto de los outliers [242] [189].

Al margen de estos dos problemas a los que se deben enfrentar los clasificadores utilizados para clasificar la señal EEG, también existen otras deficiencias propias de los sistemas BCI que deben ser destacadas. Si se pretende incorporar los sistemas BCI para el uso cotidiano fuera de los laboratorios de investigación, la autonomía es un aspecto clave que debe ser mejorado. La mayoría de sistemas BCI disponibles actualmente en el mercado [243] necesitan de un proceso de ajuste y colocación de electrodos que debe hacerse con asistencia. Además, algunos autores [134] señalan también el problema de la iniciación de los sistemas BCI. La mayor parte de estos sistemas no pueden ser iniciados de forma independiente por el usuario, sino que deben ser iniciados externamente. El problema de iniciación de un sistema BCI ha sido generalmente obviado. En algunos casos, se ha dejado la posibilidad de activación o desactivación del sistema de forma voluntaria por parte del usuario, siempre que este se encuentre en estado consciente. Los autores creen que para solucionar el problema de la iniciación, sería necesario utilizar un método basado en alguna característica que pudiera estar presente en las señales EEG durante el estado consciente y no tuviera presencia relevante con el sujeto en estado inconsciente. El uso generalizado de la tecnologia BCI también puede encontrar otro obstáculo importante en los efectos generados por la carga cognitiva. La concentración del usuario durante los experimentos que se realizan en el laboratorio, hace que no se tengan en cuenta las variables que aparecen fuera del laboratorio en forma de respuestas emocionales, fatiga, interacciones con el entorno, etc. En este sentido, diversos estudios sobre la carga cognitiva han concluido que es posible desarrollar sistemas capaces de detectar la carga de trabajo en tiempo real y actuar sobre el entorno reduciendo elementos distractores según necesidad. Cuanto mayor sea la carga mental, mayor tiempo de reacción tendrá el usuario. Por último, la variabilidad de la señal EEG, no sólo entre distintos sujetos sino también entre ensayos realizados por un mismo sujeto, puede generar altas probabilidades de error. La presencia de artefactos, descritos en el capítulo anterior, generan potenciales muy potentes y difíciles de controlar que, si no se detectan, pueden empeorar de forma drástica la tasa de acierto. 
Además de todo lo anterior, los retos a los que las investigaciones en BCI con señales EEG deben enfrentarse en la actualidad, se sintetizan en tres aspectos clave, enunciados en la literatura cientifica [244]:

1. Respecto de la calidad de los datos, cabe preguntarse: ¿es la tarea ejecutada independiente de la respuesta obtenida?

2. Atendiendo a la capacidad de generalización o al posible sobreentrenamiento del sistema, debe analizarse: ¿los resultados offline pueden generalizarse para el caso de experimentos realizados de forma online?

3. Ante la existencia de retroalimentación, es necesario preguntarse: ¿los métodos desarrollados con datos recogidos sin retroalimentación, tendrán el mismo rendimiento en presencia de esta?

Durante la planificación, ejecución y seguimiento de cualquier experimento BCI, deben plantearse estas cuestiones.

\subsubsection{Comparativa de algoritmos BCI}

Cada uno de los apartados anteriores ha descrito distintos experimentos BCI basados en diferentes algoritmos de clasificación para la detección de los patrones de la señal EEG. Los algoritmos de clasificación expuestos poseen evidentes diferencias que pueden generar un rendimiento óptimo bajo determinadas circunstancias, o un mal funcionamiento en otros casos. Para maximizar la precisión en la clasificación debe tenerse en cuenta el tipo de experimento ejecutado y las características de la señal EEG. En función de estos dos elementos, debe seleccionarse un algoritmo de clasificación para maximizar el rendimiento del sistema BCI. La Figura 3.14 muestra las características principales de los clasificadores más utilizados en experimentos BCI.

Existe también la posibilidad de utilizar varios clasificadores durante el proceso de decodificación, combinando algoritmos en sistemas BCI. Es posible utilizar varios clasificadores en cascada, en cuyo caso cada uno de los clasificadores se centra en los errores de clasificación cometidos por el inmediatamente anterior. De esta forma, 


\begin{tabular}{|c|c|c|c|c|c|c|c|c|c|}
\hline & Lineal & $\begin{array}{l}\text { No } \\
\text { Lineal }\end{array}$ & Generativo & Discriminante & Dinámico & Estático & Regularizado & Estable & $\begin{array}{l}\text { No } \\
\text { estable }\end{array}$ \\
\hline MLP & & $x$ & & $x$ & & $x$ & & & $x$ \\
\hline k-NN & & $x$ & & $x$ & & $x$ & & & $x$ \\
\hline $\begin{array}{l}\text { Bayes } \\
\text { cuadrático }\end{array}$ & & $x$ & $x$ & & & $x$ & & & $x$ \\
\hline HMM & & $x$ & $x$ & & $x$ & & & & $x$ \\
\hline RLDA & $x$ & & & $x$ & & $x$ & $x$ & $x$ & \\
\hline \multicolumn{10}{|l|}{ Perceptrón } \\
\hline RBF-SVM & & $x$ & & $x$ & & $x$ & $x$ & $x$ & \\
\hline
\end{tabular}

Figura 3.14: Propiedades de los clasificadores utilizados comunmente en los experimentos BCI (adaptado de [134])

es posible construir un clasificador con un buen rendimiento a partir de otros clasificadores más débiles. Sin embargo, la forma más popular de combinación de los clasificadores BCI es el voto [245] [246], debido a la simpleza de su uso y a los buenos resultados obtenidos.

\subsubsection{Algoritmos Genéticos}

Los algoritmos genéticos son una subclase de los algoritmos evolutivos donde los elementos del espacio de búsqueda son cadenas binarias u otros tipos de arrays de caracteres elementales [247]. Se trata de métodos de búsqueda adaptativos, que se inspiran en los procesos evolutivos biológicos y en los procesos de selección natural para aplicarlos al ámbito de la computación. Los algoritmos genéticos utilizan el método evolutivo para generar nuevos individuos partiendo de una población inicial. En la naturaleza, a medida que se va avanzando y se pasa de una generación a otra, las poblaciones van evolucionando de acuerdo a los principios evolutivos de la selección natural y el mantenimiento de aquellos individuos mejor adaptados. El funcionamiento de los algoritmos genéticos es similar y ha sido ampliamente descrito en el pasado por diversos autores [248] [249] [250]. Estos algoritmos disponen de una población de individuos que se corresponden con las distintas soluciones disponibles al problema planteado. Cada una de las posibles soluciones tendrá asignado un valor directamente relacionado con el grado de cercanía a la solución óptima del problema. Una mayor cercanía a la solución óptima del problema implica una mayor probabilidad de que dicho individuo (solución) sea seleccionado para reproducirse y genere descendientes tras la combinación con otro individuo. Los nuevos individuos 


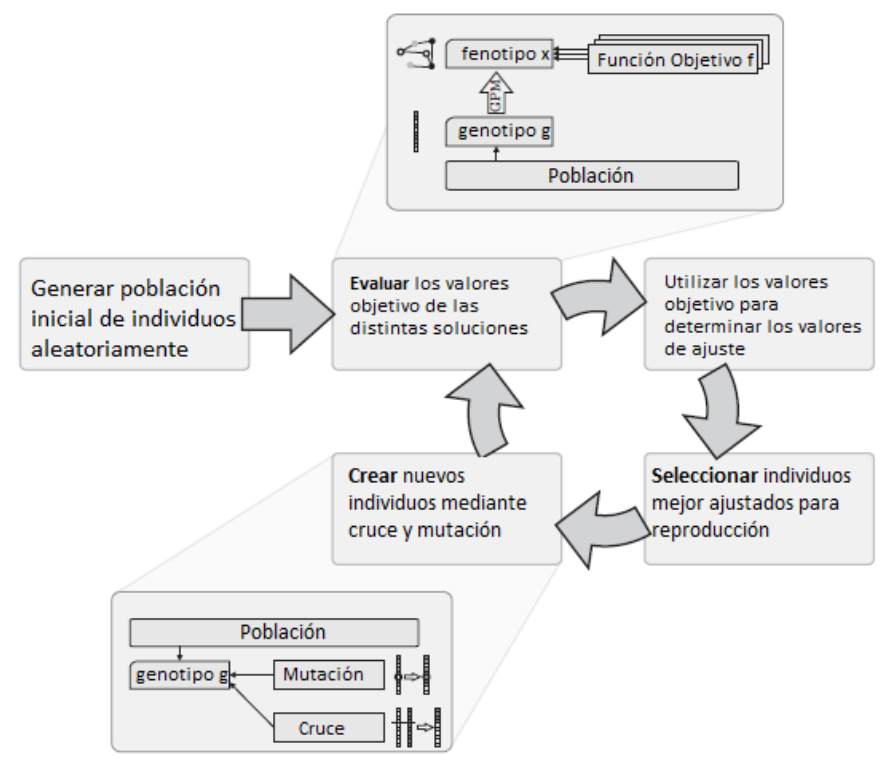

Figura 3.15: El ciclo básico de un algoritmo genético (adaptado de [247])

obtenidos en esta nueva época constituyen nuevas soluciones al problema planteado que comparten algunos de los rasgos de aquellos individuos de los que proceden. Por tanto, se genera una nueva terna de posibles soluciones al problema dado, que está más cerca que sus antecesores de la solución óptima y presentan por tanto una mejor adaptación. De esta forma, las buenas características se propagan a través del cruce de aquellos individuos mejor adaptados, más cercanos a la solución óptima. Tras un número suficiente de iteraciones, la población convergerá hacia una solución óptima del problema. Los algoritmos genéticos tienen su inicio en la decada de los 50, de la mano de un biólogo y un informático que comenzaron a aplicar simulaciones asistidas por computador con el objetivo de mejorar el conocimiento de los procesos genéticos, la selección natural y la evolución. Fue en la universidad de Michigan donde Holland [251] [252] estableció los principios básicos de los algoritmos genéticos que darían lugar a posteriores desarrollos.

Tal y como demuestra la literatura cientifica [253], los algoritmos genéticos pueden utilizarse para obtener un subconjunto óptimo de características. La selección de características en el contexto del análisis EEG se presenta como un problema de optimización multicriterio. En este sentido, los algoritmos evolutivos ofrecen una forma particularmente atractiva para abordar estos problemas de optimización. Los 
resultados obtenidos en el estudio referido, confirman que es posible utilizar algoritmos genéticos para obtener un subconjunto óptimo de características relevantes. La utilización de algoritmos genéticos para este fin no está asentada en asunciones tradicionales basadas en la monotonía, hecho que suelen limitar su aplicabilidad en problemas reales de clasificación u obtención de información.

Cuanto menor sea la adaptación de un individuo, menor será la probabilidad de que dicho individuo sea seleccionado para la reproducción, y por tanto de que su material genético se propague en sucesivas generaciones [254].

De esta manera se produce una nueva población de posibles soluciones, la cual reemplaza a la anterior y verifica la interesante propiedad de que contiene una mayor proporción de buenas características en comparación con la población anterior. Así a lo largo de las generaciones las buenas características se propagan a través de la población. Favoreciendo el cruce de los individuos mejor adaptados, van siendo exploradas las áreas más prometedoras del espacio de búsqueda. Si el Algoritmo Genético ha sido bien diseñado, la población convergerá hacia una solución óptima del problema. El poder de los Algoritmos Genéticos proviene del hecho de que se trata de una técnica robusta, y pueden tratar con éxito una gran variedad de problemas provenientes de diferentes áreas, incluyendo aquellos en los que otros métodos encuentran dificultades. Si bien no se garantiza que el Algoritmo Genético encuentre la solución óptima del problema, existe evidencia empírica de que se encuentran soluciones de un nivel aceptable, en un tiempo competitivo con el resto de algoritmos de optimización combinatoria. En el caso de que existan técnicas especializadas para resolver un determinado problema, lo más probable es que superen al Algoritmo Genético, tanto en rapidez como en eficacia. El gran campo de aplicación de los Algoritmos Genéticos se relaciona con aquellos problemas para los cuales no existen técnicas especializadas. Incluso en el caso en que dichas técnicas existan, y funcionen bien, pueden efectuarse mejoras de las mismas hibridándolas con los Algoritmos Genéticos.

Independientemente del problema al que se aplique un algoritmo genético, todos ellos tienen una serie de características comunes. Todo algoritmo genético posee una función de ajuste (fitness) que es la encargada de comprobar las distintas soluciones posibles y asignar un valor a cada una de ellas en función de su adaptación y su cer- 
canía a la solución óptima. Esta función de "fitness"parte del conjunto de soluciones posibles para el problema planteado. En este sentido, juegan un papel fundamental los procedimientos genéticos encargados de cruzar individuos entre si para generar nuevos individuos a partir de los anteriores. El proceso de búsqueda genética es iterativo: evaluar, seleccionar y recombinar la cadena de la población durante cada una de las iteraciones o generaciones hasta que se alcance una condición de terminación concreta. La evaluación de cada cadena está basada en la función de "fitness"que depende del problema. Esta función determina cuales de las soluciones candidatas son mejores. Esto corresponde con la determinación ambiental de la supervivencia en la selección natural. Las mutaciones en un algoritmo genético se producen al igual que en los sistemas naturales, son poco probables e introducen pequeños cambios. El propósito de las mutaciones es introducir nuevo material genético en un sujeto existente. Con esto se consigue añadir diversidad a las características genéticas de la población. La mutación es utilizada para asegurar que se cubre el rango total de posibilidades accesibles por cada gen, tal y como se describe en la literatura [255]. Los individuos o posibles soluciones del problema se representan a través de cadenas binarias de dígitos. A los individuos o soluciones también se les llama cromosomas. Sirva como ejemplo el estudio anteriormente mencionado, en el que se codifican un conjunto de características en una cadena numérica con elementos binarios. En esta cadena se indica con un 0 que la características que ocupa ese lugar debe ser omitida, tomando el valor 1 en caso de que deba ser incluida. Este esquema de codificación representa la presencia o ausencia de una caracteristica particular del espacio de características.

Un algoritmo genético básico comienza con una población de posibles soluciones al problema dado, que generalmente se crea de forma aleatoria. Es deseable que los individuos de la población inicial cubran un rango suficientemente grande de posibles soluciones con el objetivo de asegurar la obtención de la solución óptima, tras la finalización del algoritmo genético. Cada uno de los individuos de esta población inicial debe ser evaluado según la función de "fitness" que determinará la bondad de cada una de las soluciones respecto del problema propuesto. A través de esta función se le asigna un valor a cada individuo directamente relacionado con su cercanía a la solución óptima del problema. La correcta evaluación de cada uno de los individuos 
constituye uno de los puntos clave del algoritmo genético, de ahí la importancia de la función de ajuste. Una vez asignado un valor a todos y cada uno de los individuos, se seleccionan los mejores individuos de la población para su cruce y reproducción, con el objetivo de crear nuevos individuos que contengan las mejores características de sus antecesores. En este punto, la selección de los mejores individuos no se hace siempre siguiendo un patrón concreto, sino que existen distintos métodos de selección de individuos, aunque el objetivo final de todos ellos es descartar a los individuos con peor adaptación. Este paso es el que hace avanzar al algoritmo a través de distintas épocas, abriendo o cerrando el espacio de búsqueda de la solución óptima. La generación de nuevos individuos a partir de sus antecesores se realiza mediante la combinación del material genético de dos individuos de la época anterior. A este respecto cabe destacar que el material genético de estos individuos puede combinarse de formas muy distintas: modificando la cadena genética del individuo de forma aleatoria, combinando los cromosomas de sus antecesores para crear nuevos individuos, creando recombinaciones del material genético partiendo de dos individuos anteriores, etc. Es posible condicionar la terminación de un algoritmo genético en función de las necesidades del problema a resolver. Un algoritmo genético puede terminar porque se ha alcanzado la solución óptima o bien porque se han impuesto condiciones para la terminación del algoritmo como la limitación del número de iteraciones.

Cuanto menor sea la adaptación de un individuo, menor será la probabilidad de que dicho individuo sea seleccionado para la reproducción, y por tanto de que su material genético se propague en sucesivas generaciones [254] (Figura 3.16).

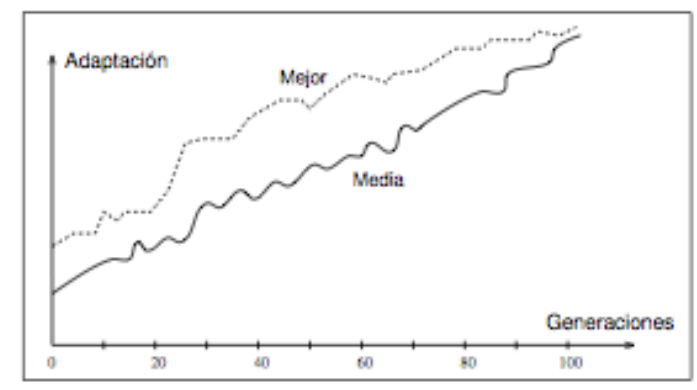

Figura 3.16: Adaptación media y mejor adaptación en un algoritmo genético simple [254]

Los algoritmos genéticos han sido utilizados en el pasado para resolver infini- 
dad de problemas de optimización en campos como el diseño de dispositivos electromagnéticos [256] o la optimización de la geometria molecular [257] entre otros muchos. También en el ámbito del BCI se han utilizado algoritmos genéticos para mejorar el rendimiento global de estos sistemas. Algunos autores [258] han implementado un sistema BCI basado en P300 que utiliza un algoritmo genético para detectar la onda P300. Este sistema fue probado de forma online en tres sujetos obteniéndose un buen funcionamiento del algoritmo genético con unos resultados de entre el $60 \%$ y el $90 \%$ en la precisión de la decodificación. Otros experimentos [259] han hecho uso de algoritmos genéticos como ayuda tanto para el proceso de selección de características como para el proceso de clasificación. Los autores de este estudio argumentan que los resultados confirman la importancia de seleccionar ciertos canales EEG y utilizar estos algoritmos para cancelar aquellas componentes que no aportan información útil al proceso de decisión.

Los algoritmos genéticos también han sido utilizados para tareas de clasificación de los potenciales generados a partir de movimientos del sujeto. En los sistemas BCI basados en potenciales relativos a movimientos, conseguir la correspondencia entre la señal generada y el movimiento ejecutado es una tarea crucial. Hace una decada, algunos autores [241] presentaron como algo muy novedoso la posibilidad de seleccionar una serie de características extraidas de una larga lista a través de un algoritmo genético. Este estudio muestra que es posible diferenciar entre movimiento contralateral de los dedos con una precisión en la clasificación de un $77 \%$ utilizando un pequeño número de características (10-20) seleccionadas de una terna de 1000 posibles características.

En el caso de los sistemas BCI asíncronos, tal y como se ha descrito anteriormente, se encargan de monitorizar de forma continua las señales cerebrales y se activan únicamente cuando el usuario intenta controlarlo. Hace algunos años, un experimento basado en un sistema BCI asíncrono [260] mostraba resultados prometedores, a pesar de que las tasas de error para la mayoría de aplicaciones prácticas eran todavía demasiado altas. Con el objetivo de mejorar su funcionamiento, los autores propusieron un modelo de adaptación al usuario. En este sentido se aplicó un algoritmo genético híbrido consistente en un algoritmo genético seguido de una búsqueda local, para personalizar el tamaño de la ventana . La aplicación de este esquema ha 
mostrado una mejora significativa de los resultados. Tras la aplicación del algoritmo genético híbrido se pasó de un $65 \%$ a un $76 \%$, en el caso del primer sujeto, y de un $53 \%$ a un $63 \%$ en el caso del segundo sujeto analizado, una mejora que demuestra el potencial de aplicación de los algoritmos genéticos en el camino para maximizar la precisión de los sistemas BCI.

\subsection{Conclusiones}

Las principales características de los sistemas BCI han sido descritas en este capítulo. Tal y como se ha puesto de manifiesto, los investigadores se han enfrentado a no pocas dificultades para comparar las tecnicas BCI descritas durante estos últimos años en la literatura cientifica. Como respuesta a esta situación, se ha venido trabajando para establecer métodos objetivos que permitan comparar entre sí sistemas BCI distintos. Al tratarse de un campo de investigación muy joven, que se está desarrollando en un entorno multidisciplinar, la comunidad BCI dispone de un lenguaje propio. Sin embargo, no existe un modelo funcional común para describir un sistema BCI que posibilite la comparación de tecnologias BCI con otros sistemas o interfaces de usuario no basadas en BCI. En este capítulo, se han abordado las diferentes formas de clasificar los sistemas BCI y sus modos de funcionamiento, en base al tipo de señal captada, a la sincronía o asincronía del proceso, a si el usuario ejerce un control directo sobre el sistema BCI o no, etc. También se ha analizado si se trata de un sistema BCI espontáneo o de potenciales evocados.

Se han descrito y comparado los diferentes algoritmos de clasificación utilizados para diseñar sistemas BCI. Los algoritmos mencionados pueden encontrarse con mucha facilidad en la literatura científica como base de la mayoria de experimentos $\mathrm{BCI}$ realizados en los últimos años. Analizando el comportamiento de cada uno de estos algoritmos en experimentos BCI concretos, pueden extraerse interesantes conclusiones que ponen de manifiesto dos aspectos clave en el diseño de sistemas BCI:

1. La necesidad de definir previamente las necesidades o prestaciones del sistema BCI para seleccionar el modo de funcionamiento óptimo del sistema en función de la tarea a realizar, la naturaleza de la señal, la sincronía del proceso, etc. 
2. La importancia de seleccionar el algoritmo adecuado para minimizar el error de clasificación durante el proceso de análisis, detección y clasificación de la señal EEG.

Los experimentos referenciados en este capítulo, arrojan resultados concluyentes sobre los factores que influyen en la precisión de la clasificación del sistema. Es fundamental disponer de información sobre las necesidades del sistema BCI en el momento de elegir o diseñar un clasificador para dicho sistema. Durante el proceso de diseño del sistema BCI se han de tener en cuenta los problemas a los que se enfrentará el clasificador en un contexto como el planteado. Si el número de muestras disponibles es demasiado pequeño en comparación con el número de dimensiones o vectores de características, el clasificador tendrá dificultades para arrojar buenos resultados debido a la "maldición de la dimensionalidad". Este es el reto más importante, de entre los mencionados anteriormente, a los que se enfrentan los clasificadores utilizados en sistemas BCI para reducir al mínimo el error, debido a la naturaleza de la señal EEG. A pesar de que los clasificadores lineales son generalmente más robustos que sus clasificadores homólogos no lineales, en presencia de fuerte ruido y datos atípicos, también los sistemas lineales pueden resultar poco precisos. Durante este capítulo ha quedado demostrado el gran potencial de los algoritmos de regularización en enrotnos BCI. Con la aplicación de estos algoritmos sería posible limitar la influencia de los datos atípicos y reducir la complejidad del clasificador. En este sentido, también SVM presenta buenos resultados de clasificación, debido fundamentalmente a su inmunidad ante la "maldición de la dimensionalidad". Otros algoritmos descritos también pueden presentar buenos resultados en función del escenario al que se enfrenten. Por tanto, escoger un clasificador adecuado y adaptado a las características de las señales que deben ser analizadas es el aspecto clave a la hora de diseñar con éxito un sistema BCI.

El objetivo principal de esta tesis está centrada en demostrar la viabilidad de decodificar y clasificar señales EEG durante la ejecución de tareas motoras en el espacio tridimensional. Durante la clasificación se dispondrá de un número limitado de muestras con una alta dimensionalidad. Para conseguir altas precisiones en la decodificación de los movimientos tridimensionales a partir de señales EEG, se desarollará un sistema BCI optimizado a partir de las conclusiones obtenidas en este 
capítulo. El algoritmo seleccionado deberá estar optimizado para superar la maldición de la dimensionalidad, reducir el tiempo de clasificación de los datos y conseguir altas precisiones. Las conclusiones de este capítulo tras el análisis de los distintos algoritmos ponen de manifiesto la superioridad computacional con la que cuenta el algoritmo RLDA para experimentos BCI complejos. Ha quedado demostrado que los métodos de clasificación lineales son una buena opción para desarrollar experimentos BCI tanto por su simplicidad como por su rendimiento. El algoritmo RLDA aporta una solución efectiva para el problema de la singularidad, mejorando el funcionamiento del algoritmo LDA en terminos de precisión, especialmente en aquellos casos en los que se dispone de un número pequeño de muestras de entrenamiento. En casos en los que se disponga de unas pocas muestras con una gran dimensionalidad, la regularización del algoritmo RLDA permitira evitar problemas de sobreentrenamiento que pudieran limitar la capacidad de generalización.

Está fuera de toda duda que algunos de los resultados mencionados durante este capítulo reflejan unos claros avances teóricos y prácticos en el campo del BCI. Sin embargo, la alta variabilidad entre sujetos y la baja velocidad de transmisión de información, limitan el desarrollo de los sistemas BCI tanto para aplicaciones clínicas como para potenciales aplicaciones fuera del laboratorio. La bibliografia referenciada durante todo el capítulo utiliza algunas tareas específicas como correlación de la intención del usuario. Se utilizan, por ejemplo, movimientos imaginarios para tomar decisiones en base a la correspondencia con uno u otro movimiento. Hasta ahora, se han desarrollado un número importante de investigaciones cuyo objetivo era generar avances del estado del arte relacionados con cada uno de los pasos del ciclo de funcionamiento de un sistema BCI (Figura 3.1). Sin embargo, cada vez está más extendida la necesidad de orientar los sistemas BCI hacia aplicaciones reales, fuera de las condiciones ideales que proporcionan los laboratorios de investigación. Uno de los objetivos de esta tesis, es el desarrollo de aplicaciones prácticas tanto para personas sanas como con posibles discapacidades. En los últimos años se ha generado un interés creciente en torno a la tecnología desarrollada alrededor del BCI. En el pasado, el desarrollo del BCI ha estado centrado en mejorar las condiciones de interacción con el entorno de las personas discapacitadas y con problemas motores. Por tanto, los sistemas BCI surgen por la necesidad de establecer nuevos canales de 
comunicación para personas con discapacidades graves. Sin embargo, para su pleno desarrollo algunas empresas como Neurosky [261] o Emotiv [262] han enfocado su modelo de negocio a partir del BCI aplicado al mundo del vídeojuego. Debido fundamentalmente al gran potencial existente en el número de usuarios. En este sentido, Las mejoras que se han producido durante estos últimos años han hecho que la tecnología BCI comience a ser considerada para su uso en otro tipo de aplicaciones. Este cambio parcial en el público objetivo, hace que en esta nueva línea sea imprescindible definir las necesidades y características de los sistemas BCI para estas nuevas aplicaciones que serán utilizados por sujetos sanos para aplicaciones totalmente distintas que aquellos sujetos con problemas motores o cognitivos que utilizan esta tecnologia como herramienta para mejorar sus condiciones de vida. En los capítulos siguientes se profundiza en estos aspectos del BCI. Se analizará por un lado el rendimiento de los sistemas BCI en entornos tridimensionales, para desarrollar una propuesta de aplicación práctica de un sistema BCI orientado al uso fuera de los laboratorios de investigación. 


\title{
Capítulo 4
}

\section{Decodificación y clasificación de la señal EEG en entornos tridimensionales}

\author{
Tengo buenas noticias. Tendrás la \\ oportunidad de ser inmortal. Mientras nada \\ le ocurra a ese bonito cerebro.
}

Robocop 2

\subsection{Introducción}

Estudios recientes sobre la decodificación neuronal del movimiento o la intención de movimiento basado en señales electroencefalográficas (EEG) [263-266] [13] [14] [267] han mostrado que las señales de EEG contienen información útil sobre el sistema motor que puede ser extraída, analizada y clasificada. Estos estudios mostraron que la decodificación de las trayectorias puede realizarse con la observación de las fluctuaciones obtenidas a partir de un filtro paso bajo (modulación de amplitud, o AM) de las señales de EEG o de las modulaciones espectrales en la banda delta $(\delta)$ $(0,1-4 \mathrm{~Hz})$. Además, la viabilidad de la decodificación continua de los movimientos de la mano iniciados por el propio sujeto y desarrollados en el espacio tridimensional, a partir de información electroencefalografica utilizando decodificadores lineales ha 
sido recientemente verificada [265]. En general, los decodificadores neuronales pueden ser diseñados para predecir, en base al análisis de la señal EEG, las estimaciones de múltiples estados internos tales como dirección de movimiento hacia determinados puntos en el espacio [263] [264] o para predecir variables continuas de tiempo asociadas al punto final de la trayectoria recorrida por la mano [13]. Estas referencias pueden servir como señales de referencia a un dispositivo protésico colocado en una extremidad inferior o superior, el cursor de una computadora, un dispositivo de deletreo o un teclado virtual; en definitiva, a un sistema BCI. Uno de los objetivos de las investigaciones para la mejora del BCI es el desarrollo de sistemas capaces de decodificar las señales cerebrales generadas tras la planificación y/o ejecución de movimientos naturales. La comprensión de los límites sobre la información EEG que puede extraerse de la corteza cerebral con respecto al número de grados de libertad, la complejidad, fiabilidad, velocidad, y el nivel de control intuitivo que se puede lograr, son aspectos clave en las investigaciones actuales del BCI.

Tal y como se ha comentado anteriormente, los sistemas BCI traducen las señales cerebrales del individuo en productos funcionales para el control o la comunicación directa con un dispositivo final. Debido a que este tipo de comunicación no depende de los nervios periféricos y los músculos, el desarrollo de sistemas no invasivos se ha centrado en las solicitudes de las personas que están gravemente paralizadas para restablecer la comunicación y la interacción con su entorno. Desafortunadamente, los sistemas BCI todavía poseen algunas limitaciones mencionadas en el Capítulo 2. En este sentido, algunos de los sistemas BCI basados en EEG requieren semanas o meses de entrenamiento antes de que los sujetos puedan alcanzar un cierto nivel de control cerebral y sólo son capaces de desarrollar velocidades de comunicación lentas. El tiempo de entrenamiento y la velocidad en bits por segundo son los principales determinantes de la aceptación de la tecnología BCI de los usuarios potenciales. Como se verá en apartados siguientes, en el presente trabajo se desarrolla un sistema BCI capaz de mejorar sustancialmente ambos parámetros.

Hace unos años, algunos autores [268] comenzaron a mostrar la posibilidad de controlar movimientos en el espacio tridimensional mediante un sistema BCI. Este estudio muestra que una alta resulución de señal no es un aspecto esencial para el control de movimientos complejos, y sugiere que existen otros factores más impor- 
tantes a tener en cuenta. Los autores lanzan una hipótesis, argumentando que la combinación de señales de múltiples áreas corticales podrían producir un funcionamiento mucho más consistente. Con desarrollo posterior, argumentan los autores, podría ser posible que personas con desórdenes neuromusculares severos pudieran operar, a través de sus señales cerebrales, dispositivos como brazos robóticos, sillas de ruedas motorizadas o neuroprótesis.

El rendimiento limitado de los actuales EEG basados en sistemas BCI puede estar relacionado con la elección de características (P300, ritmos motosensoriales, etc.), y/o con el uso de señales neuronales estereotipadas y no específicas para provocar salidas del sistema predefinidas. Sin embargo, estudios recientes sugieren que las amplitudes $(<4 \mathrm{~Hz})$ de señales EEG en toda la corteza cerebral contiene información detallada acerca de la representación del movimiento en la macro escala, que podría conducir a mejoras en la velocidad de comunicación y tiempo de entrenamiento. En este sentido [264], se investigó la actividad EEG relativa a aquellos casos en los que los targets eran seleccionados por el propio sujeto. Bajo estas condiciones se mostró que la dirección del movimiento de la mano puede deducirse, a través de un solo ensayo, usando un filtro paso-bajo $(3 \mathrm{~Hz})$ el cual, mide la actividad neuronal con más de 20 sensores distribuidos sobre la corteza cerebral. Utilizando un sistema EEG de alta densidad y un conjunto de ocho vectores de características diferentes, algunos autores [263] mostraron que es viable decodificar la intencionalidad (durante la planificación motora) del movimiento de la mano para alcanzar un objetivo (uno de cuatro objetivos en el espacio 2D) y la dirección del movimiento de la mano durante la trayectoria real (uno de tres objetivos en 3D) a partir de 140-150 canales EEG.

Agunos autores [264], han comparado la precisión en la decodificación (DA) y la información mutua de shannon ${ }^{1}$ entre la dirección del movimiento decodificada y la medida (definida como información decodificada (DI) en bits) en los estudios anteriormente referenciados. Sin embargo, no está del todo claro cómo un número mayor de objetivos a alcanzar en los experimentos puede acabar afectando a la precisión de decodificación. Por tanto, el número óptimo de objetivos para maximizar la precisión de decodificación (DA) y la información decodificada (DI) es desconocido.

\footnotetext{
${ }^{1}$ comprobar que es correcta la traducción Shannon's mutual information
} 
En este estudio, se evalúan DA y DI durante movimientos autoiniciados de la mano hacia uno de los ocho objetivos. Estos objetivos han sido seleccionados por el propio sujeto y están dispuestos en un espacio tridimensional.

Con este trabajo se pretende demostrar que es posible obtener altas precisiones en la decodificación de las señales cerebrales asociadas a movimientos voluntarios de las extremidades superiores del sujeto en entornos tridimensionales. Para realizar una comparativa entre los resultados que arrojan los diferentes estudios, han de tenerse en cuenta otros parámetros a la hora de evaluar si el porcentaje de precisión en la decodificación es suficientemente bueno o no. Es evidente que para comparar el porcentaje de precisión en la decodificación en un determinado experimento se debe tener en cuenta el número de targets, si el sistema es invasivo o no y/o el número de electrodos del sistema electroencefalográfico. Para ello, utilizaremos un parámetro que permite relacionar y comparar experimentos BCI teniendo en cuenta los parámetros anteriormente mencionados. El rendimiento en la decodificación puede cuantificarse mediante la precisión en la decodificación (DA), definida como el porcentaje de ensayos decodificados correctamente. A partir de la DA, es posible obtener la información decodificada (DI), parámetro que cuantifica la cantidad de información extraida sobre la dirección de movimiento [269]. La información decodificada (DI) ha sido definida como la información mutua entre el movimiento decodificado y la dirección real del movimiento. Asumiendo igualdad de probabilidades de predicciones correctas para cada dirección e igual distribución para las predicciones erróneas de todas las direcciones, la relación entre DI y DA puede expresarse de la siguiente forma [26]:

$$
D I(D A)=\frac{D A}{100} \log _{2} \frac{D A}{D A_{\text {chance }}}+\frac{100-D A}{100} \log _{2} \frac{100-D A}{100-D A_{\text {chanche }}}
$$

De esta forma, conociendo la precisión obtenida en el proceso de decodificación de un experimento concreto y el número de targets, es posible obtener la información decodificada en dicho esperimento. El número de targets vendrá dado por $D A_{\text {chance }}$, cuyo valor se obtiene del cociente: $D A_{\text {chance }}=\frac{100}{N_{\text {Targets }}} \%$, donde $N_{\text {Targets }}$ es el número de targets utilizados. Esto permitirá comparar los resultados obtenidos en el presente estudio con los resultados de otros sistemas BCI, aún cuando se trate de experimentos que han utilizado diferente número de targets. 


\subsection{Materiales y métodos}

Para llevar a cabo el procedimiento experimental, la Junta de Revisión Institucional de la Universidad de Maryland en "College Park" aprobó un protocolo experimental. Después de que dieran su consentimiento, cinco sujetos sanos y diestros fueron colocados en una silla, invitando a todos ellos a ejecutar por iniciativa propia, trayectorias desde un punto central hacia objetivos o targets externos, distribuidos en su espacio visual y en un entorno tridimensional. La distancia desde la posición central para cada uno de los objetivos era de $22 \mathrm{~cm}$ aproximadamente y la estructura utilizada en este experimento se muestra en la Figura 4.1.

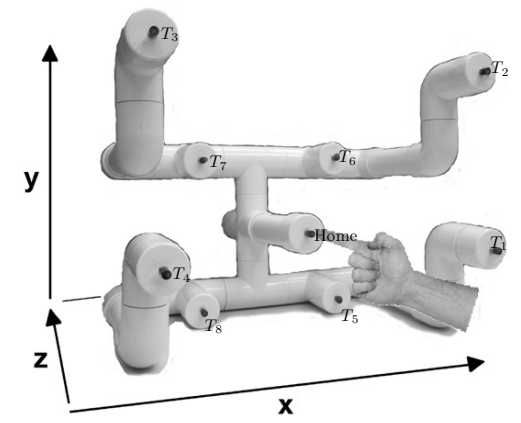

Figura 4.1: Estructura utilizada en el experimento con la numeración de cada uno de los targets que el sujeto puede seleccionar y el punto central de inicio

Antes de comenzar con la adquisición de señales EEG, se instruyó a los sujetos para hacer selecciones aleatorias de los ocho objetivos sin contar las veces que hacían la selección. Durante el tiempo en el que los sujetos ejecutaban la trayectoria, fijaban su mirada en un LED, parpadeando únicamente cuando su mano descansara en el destino. El experimento se consideró finalizado una vez que la trayectoria hasta cada uno de los ocho objetivos fue realizada al menos en 10 ocasiones. La recogida de datos se llevó a cabo mediante un casco EEG de 58 electrodos que se colocó sobre la cabeza de los sujetos de acuerdo con el sistema internacional 10-20 (Figura 4.2). Las señales de EEG fueron muestreados a $1000 \mathrm{~Hz}$, filtradas por un paso-banda de 0,5 a $100 \mathrm{~Hz}$. La actividad electro ocular horizontal y vertical se midió mediante sensores bipolares. Los datos del EEG fueron diezmados desde $1 \mathrm{kHz}$ a $100 \mathrm{~Hz}$ y filtrados mediante un filtro paso-bajo y filtro anti-aliasing con una frecuencia de corte de 40 $\mathrm{Hz}$. La trayectoria seguida por el brazo del sujeto fue monitorizada y grabada en 


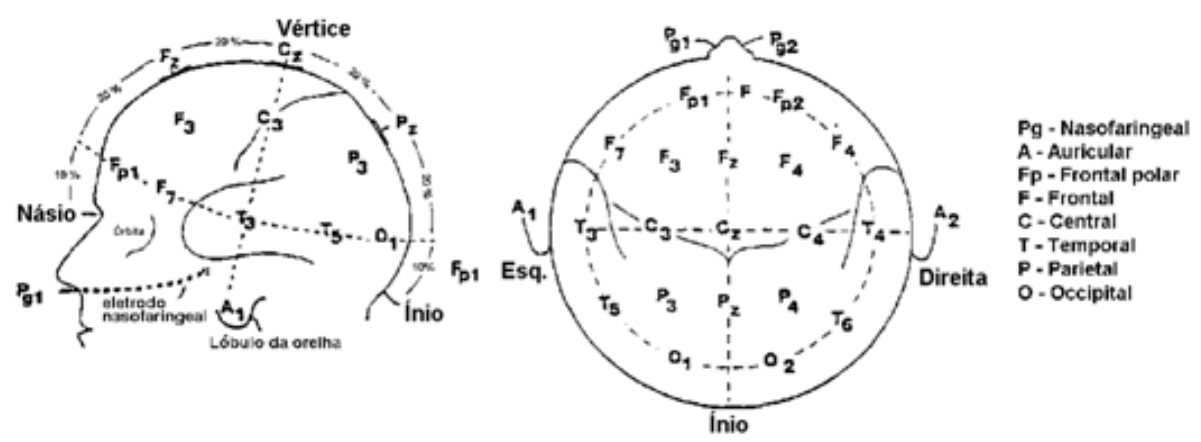

Figura 4.2: El sistema internacional 10-20. Los electrodos se encuentran espaciados entre el $10 \%$ y el $20 \%$ de la distancia total entre puntos reconocibles en el cráneo. Estos puntos clave, a partir de los cuales se realizan las medidas, son: "Naison" que corresponde con la indentación entre la frente y nariz. Ïnión", que se corresponde con la protuberancia occipital y el "punto preauricular", que se encuentra delante del trago de cada pabellón de la oreja [270] [271]

todo momento tomando muestras a $100 \mathrm{~Hz}$, utilizando un sistema de detección de movimiento que seguía el desplazamiento de un LED infrarrojo colocado a la punta del dedo índice. Este sistema aporta información detallada sobre la trayectoria exacta del brazo del sujeto durante la ejecución del movimiento. Relacionando esta información con la señal EEG captada durante la ejecución del movimiento, será posible mejorar el proceso de clasificación relacionando la trayectoria espacial con la actividad cerebral decodificada por el sistema BCI. Además, la actividad electroocular horizontal y vertical fue grabada mediante la utilización de un sensor bipolar.

La elección del algoritmo de clasificación del sistema BCI se efectuará a partir del análisis de las características de las señales EEG captadas. El algoritmo elegido debe optimizar el proceso de decodificación del tipo de señales de entrada de este sistema. Cada sujeto $s$ genera una matriz $\left(X_{s i}\right)$ con las señales EEG captadas en cada uno de los intentos $i$. Las filas de esa matriz $X_{s}$ se corresponden con cada uno de los electrodos del sistema BCI y contienen la información EEG captada por cada canal en el dominio de tiempo. El número de columnas de la matriz $X_{s}$ están directamente relacionadas con el tiempo que tarda el sujeto en completar la tarea de desplazamiento del brazo. 


$$
X_{s i}=\left(\begin{array}{cccccc}
a_{11} & a_{12} & a_{13} & a_{14} & \ldots & a_{1 T} \\
a_{21} & a_{22} & a_{23} & a_{24} & \ldots & a_{2 T} \\
\vdots & \vdots & \vdots & \vdots & \vdots & \vdots \\
a_{n 1} & a_{n 2} & a_{n 3} & a_{n 4} & \ldots & a_{n T}
\end{array}\right)
$$

Cada sujeto realiza un número mínimo de intentos (10) desplazando su brazo derecho desde el centro hasta cada uno de los targets marcados en la estructura de la Figura 4.1. Por cada uno de los intentos, se obtiene una matriz como la descrita en la ecuación 4.2. Una vez que el sujeto ha realizado las trayectorias hacia todas las direcciones, se dispondrá de la matriz $X_{s i}$ para un sujeto $s$ por cada uno de los intentos $i$. Con estos datos, se construirá una matriz $X_{s}$. Esta matriz contendrá los datos EEG obtenidos en cada uno de los intentos hacia las distintas direcciones del sujeto $s$. Cada una de las filas de $X_{s}$ contendrá los datos de los distintos intentos realizados por un sujeto. Cada fila de la matriz $X_{s}$ contiene todos los datos EEG del intento $i$ realizado por el sujeto. A partir de la matriz de la ecuación 4.2 de dimensiones $N * T$ (donde $\mathrm{N}$ es el número de electrodos y $\mathrm{T}$ es la duración del intento) se construye un vector fila de dimensiones $1 x L$. Las matrices $X_{s i}$ de cada uno de los intentos se convierten en un vector fila, todos ellos juntos forman las filas de la nueva matriz $X_{s}$ de dimensiones $i x L$ donde i es el número total de intentos y $\mathrm{L}$ es la longitud de cada intento. Llegado este punto, la construcción de la matriz que aglutina los intentos totales del sujeto presenta un problema importante. Es evidente que cada sujeto utiliza un tiempo distinto en recorrer la trayectoria hasta cada uno de los objetivos desde el punto inicial. Como consecuencia, la longitud de los datos EEG obtenidos en cada uno de los intentos varían de un intento a otro. Cada una de las muestras tiene un número distinto de elementos y este hecho dificulta la construcción de la matriz $X_{s}$ (ecuación 4.3)

$$
X_{s}=\left(\begin{array}{cccccc}
a_{11} & a_{12} & a_{13} & a_{14} & \ldots & \\
a_{21} & a_{22} & a_{23} & a_{24} & a_{2 t} & \\
\vdots & \vdots & \vdots & a_{f j} & & \\
a_{n 1} & a_{n 2} & a_{n 3} & a_{n 4} & \ldots & a_{n T}
\end{array}\right)
$$

Para solucionar este problema los datos de cada intento deben ser remuestreados 
para que todos ellos posean una longitud fija, facilitando el proceso de entrenamiento del algoritmo de clasificación. Antes de comenzar este proceso, los datos del EEG fueron ordenados por dirección de destino. Posteriormente se les alineó con el inicio del movimiento (MO - Movement Onset), donde MO se define como el momento en el que el sujeto suelta la punta del dedo del interruptor de la posición inicial mostrada en la Figura 4.1. Cada uno de los intentos del sujeto desde que pulsa el botón de inicio hasta que pulsa el botón de final constituye una muestra, una fila en la matriz $X_{s}$. Se consideró como final del movimiento el momento en el que el sujeto pulsa el botón de destino ubicado en cualquiera de los ocho objetivos marcados en la Figura 4.1. Cada una de las muestras fueron sometidas a un proceso de filtrado mediante un filtro paso bajo . Una vez filtradas, las muestras fueron re-muestreadas para que todas compartiesen una longitud fija de 1,4 segundos. Los datos EEG captados durante cada uno de los intentos hacia cualquiera de las direcciones tiene una longitud temporal de 1,4 segundos de los que 0,2 segundos corresponden al periodo previo al MO mientras que los siguientes 1,2 segundos se corresponden con los datos obtenidos desde que el usuario pulsa el boton inicial (Figura 4.1) hasta que alcanza su destino. Tras este proceso, las muestras se filtraron de nuevo para extraer la información de amplitud dentro de la banda delta. Es importante destacar que la operación de re-muestreo conserva la información de patrón. Durante el proceso se mantienen las fluctuaciones en la amplitud de las señales del EEG durante el intervalo de análisis elegido, proporcionando vectores de características de entrada $(\mathrm{n}=140)$ de longitud constante tanto para el proceso de entrenamiento como de clasificación.

Una fragmento temporal de las señales utilizadas como entrada al algoritmo del clasificación puede observarse en la Figura 4.3. Se muestra el voltaje y la variación temporal de las señales captadas por los electrodos FC5, FC3, C3 y CP3. La Figura 4.4 muestra la localización espacial de cada uno de los electrodos a lo largo de la superficie de la cabeza del sujeto. Tal y como se ha descrito anteriormente, las señales fueron remuestreadas para que todas tuviesen una duración de 1.2 segundos conservando la información de patrón y las fluctuaciones en la amplitud durante este intervalo. Estas señales corresponden al periodo de tiempo en el que el sujeto desplaza el brazo desde el centro hacia cualquiera de los objetivos marcados en la 


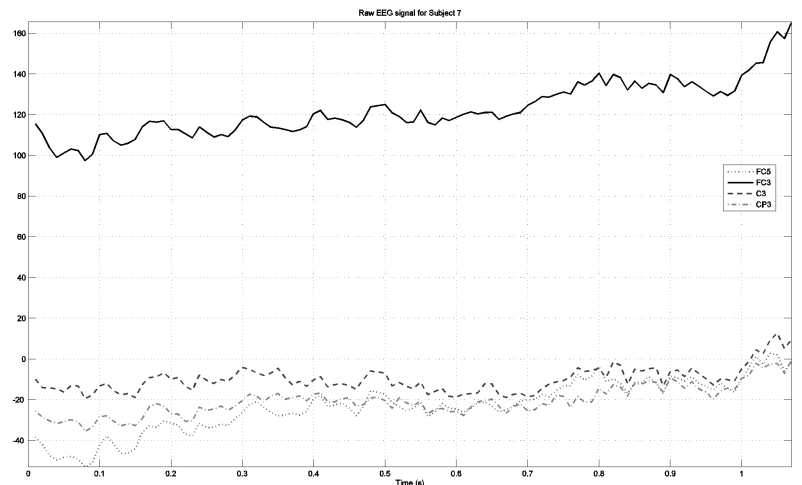

Figura 4.3: Señales captadas por los electrodos FC5, FC3, C3 y CP3. Han sido remuestreadas con el objetivo de que todas ellas posean una duración de 1.2 segundos.

\section{Figura 4.1.}

Cada fila de la matriz $X_{s}$ tiene asociada un valor de la matriz columna $Y_{s}$ (ecuación 4.4). La matriz $Y_{s}$ es un vector clumna cuyos elementos toman valores entre 1 y 8. Estos valores representan cada una de las 8 trayectorias posibles que el sujeto puede realizar y que se encuentran etiquetadas en la Figura 4.1. La muestra $i$ de la matriz $X_{s}$ estara asociada a la dirección marcada por el elemento $Y_{i}$. El sujeto $s$, al realizar el intento correspondiente a los datos contenidos en la fila $i$ de la matriz $X_{s}$ desplazo su brazo hasta el target indicado en el elemento $Y_{i}$ de la matriz 4.4.

$$
Y_{s}=\left(\begin{array}{c}
Y_{1} \\
Y_{2} \\
\vdots \\
Y_{j}
\end{array}\right)
$$

Para definir los principales parametros del algoritmo de clasificación, se utilizará una parte de las muestras de $X_{s}$ e $Y_{s}$ como conjunto de entrenamiento, dejando otra parte para utilizar como conjunto de test. Una vez entrenado el algoritmo con un número de muestras concreto definido como conjunto de entrenamiento, el resto de muestras no evaluadas todavía por el algoritmo serán clasificadas en función de las reglas del clasificador. La precisión en la decodificación (DA) será el porcentaje de muestras correctamente clasificadas. El valor de DA se obtiene comparando la matriz $Y_{s}$, que contiene las etiquetas a las que efectivamente pertenecen cada una de 


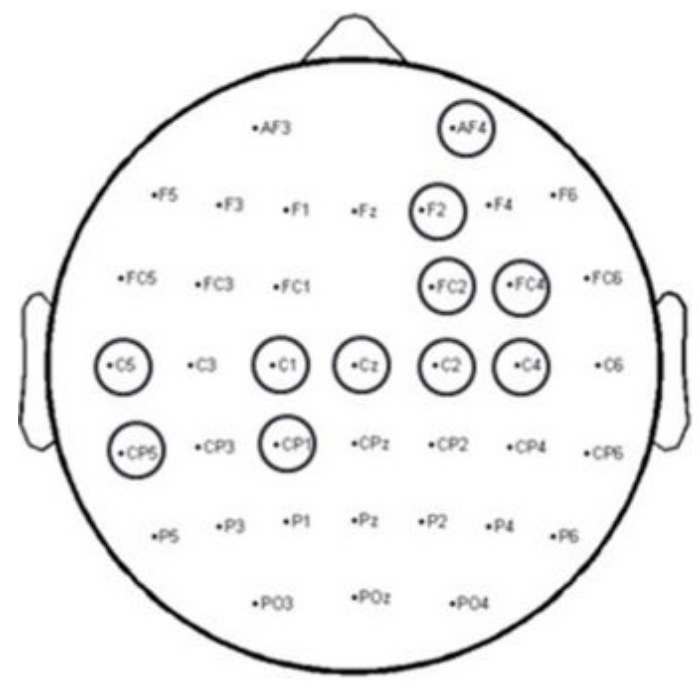

Figura 4.4: Etiquetas asignadas a cada uno de los electrodos utilizados para captar las señales EEG

las muestras, con la matriz $Y_{\text {predict }}$ que contiene las clases a las que el algoritmo ha asignado cada muestra clasificada.

\subsubsection{Método de clasificación}

Durante el proceso de clasificación de la señal EEG obtenida, han de considerarse los aspectos descritos en el Capítulo 3 para seleccionar el clasificador más adecuado. El algoritmo seleccionado deberá clasificar cada una de las muestras contenidas en la matriz $X_{s}$, relacionando cada una de las filas de la matriz con una de las ocho posibles clases (objetivos de la Figura 4.1). Cada una de las muestras de $X_{s}$ posee un número muy elevado de dimensiones en comparación con el número de muestras disponibles. Cada uno de los sujetos realizará 10 intentos para cada una de las 8 direcciones posibles en las que puede desplazar su extremidad. Dispondremos por tanto de 10 muestras con datos EEG asociados a cada una de las 8 clases entre las que el clasificador deberá escoger. Este número de muestras es muy pequeño en comparación con las dimensiones de cada muestra, situadas en el orden de $10^{5}$. En este sentido, el algoritmo LDA clásico (Classical Linear Discriminant Analysis) no es aplicable para aquellas muestras que tengan un tamaño demasiado pequeño debido a la singularidad de las matrices de dispersión involucradas. Sin embargo, 
el algoritmo LDA regularizado o RLDA, aporta una estrategia muy simple para superar el problema de la singularidad a través de la aplicación de un término de regularización $(\lambda)$, que es comunmente estimado utilizando la validación cruzada a partir de una terna de posibles valores para dicho parámetro [272]. El algoritmo LDA clásico no es efectivo en aquellos casos en los que los datos poseen un elevado número de dimensiones y el tamaño de las muestras no exceden dichas dimensiones. Este problema es posible resolverlo mediante el algoritmo RLDA propuesto [272]. En el presente trabajo se ha utilizado el algoritmo LDA regularizado (RLDA) en su implementación descrita recientemente [189] para clasificar patrones de señales en el dominio del tiempo en banda delta. El algoritmo RLDA posee las características de un algoritmo estable y regularizado, debido a que pequeñas variaciones en el conjunto de entrenamiento no afectarán de forma considerable al proceso de clasificación. Además, por el hecho de ser un algoritmo regularizado, estará dotado de una buena capacidad de generalización y una gran robustez ante la presencia de outliers. En el Capítulo 3 ya se pronosticó que en aquellos casos en los que los datos están en un espacio con gran número de dimensiones y el tamaño de las muestras no excede dicha dimensión, las matrices de dispersión involucradas pueden ser singulares. Este hecho genera el problema de la singularidad que puede ser solucionado de forma efectiva utilizando RLDA. La base de esta solución se encuentra en añadir una constante $\lambda>$ 0 a los elementos diagonales de la matriz total de dispersión; este será el parámetro de regularización que permitirá estabilizar la estimación de las muestras de la matriz de covarianza y mejorar el funcionamiento de la clasificación del algoritmo LDA. Dada las consecuencias positivas de introducir el parámetro de regularización, escoger un valor apropiado para este parámetro es crítico para el buen funcionamiento de RLDA ([188]). Un valor grande de $\lambda$ puede generar distorsiones en la información de la matriz de dispersión. Un valor pequeño de $\lambda$ puede no ser suficientemente efectivo para resolver el problema de la singularidad. El parámetro $\lambda$ será determinado en función del error de clasificación, que se obtendrá para cada valor propuesto en cada uno de los parámetros [190]. El valor óptimo para $\lambda$ no tiene por qué ser conocido de antemano. El valor óptimo será seleccionado durante el proceso de entrenamiento utilizando un conjunto de muestras para estimarlo.

El parámetro de regularización $(\lambda)$ fue seleccionado a partir de un conunto de 
posibles valores seleccionados para este experimento:

$$
\lambda=\left[10^{-10}, 10^{-7}, 10^{-4}, 0,1,10,100,10^{4}, 10^{7}, 10^{10}\right]
$$

Como entrada al clasificador RLDA se han utilizado las señales EEG de la matriz $X_{s}$ (ecuación 4.6). Cada una de las muestras (filas de la matriz) se ha clasificado utilizando dos diferentes periodos de tiempo:

1. El fragmento correspondiente al pre-movimiento del sujeto (tomando $200 \mathrm{~ms}$ de datos EEG previos al inicio del movimiento, desde $-200 \mathrm{~ms}$ hasta 0 s.). De esta forma se clasifican los datos EEG obtenidos durante los instantes inmediatamente anteriores al inicio de de la trayectoria por parte del sujeto.

2. El fragmento de datos EEG correspondiente a la duración total del movimiento, incluyendo los datos del pre-movimiento. Todo ello alineado con el inicio de movimiento (MO)

$$
X_{s}=\left(\begin{array}{cccccc}
a_{11} & a_{12} & a_{13} & a_{14} & \ldots & a_{m T} \\
a_{21} & a_{22} & a_{23} & a_{24} & \ldots & a_{m T} \\
\vdots & \vdots & \vdots & \vdots & \vdots & \vdots \\
a_{n 1} & a_{n 2} & a_{n 3} & a_{n 4} & \ldots & a_{m T}
\end{array}\right)
$$

Para determinar el valor del parámetro de regularización de forma óptima, tal y como se apuntó en el Capítulo 3, el método más utilizado es el de validación cruzada (cross-validation) [191] [192]. La validación cruzada de K-particiones (k-fold cross validation) es la más utilizada en la actualidad y consiste en dividir el conjunto de ejemplos disponibles en 'k'conjuntos de igual tamaño $T_{1}, T_{2}, \ldots, T_{k}$. En esta validación cruzada se realizan $k$ experimentos, utilizando $T_{j}$ (con $j \neq i$ ) como conjunto de entrenamiento en la iteración i-ésima, y $T_{i}$ como conjunto de test. Aplicando la validación cruzada es posible comprobar los resultados obtenidos para distintos valores del parámetro de regularización sobre distintos conjuntos de test. Escoger el valor más apropiado de los parámetros de regularización para cada situación particular es el objetivo de este proceso. Se utiliza la validación cruzada con k=10, por lo que se utilizarán 10 conjuntos de muestras para obtener el parámetro de regularización que mejores resultados aporte en términos de precisión en la decodificación (DA). 


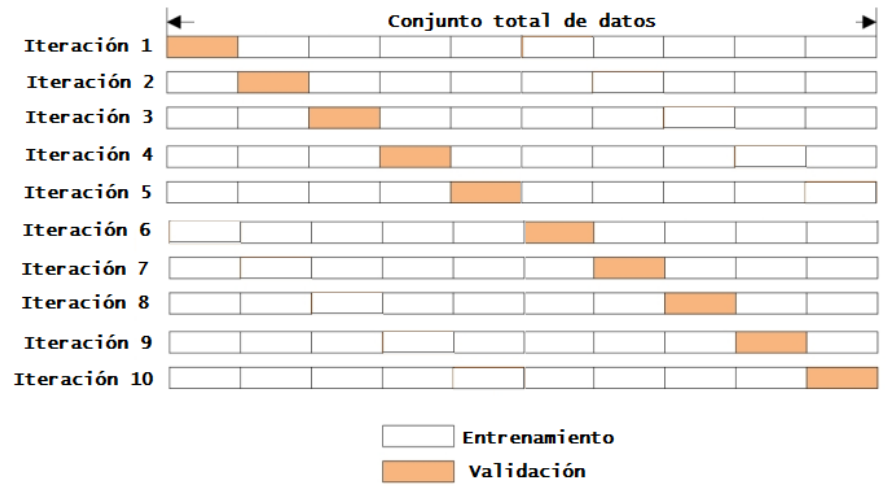

Figura 4.5: Validación cruzada con $\mathrm{k}=10$. Se utilizan parte de las muestras para entrenar el algoritmo y parte de ellas para verificar el resultado del entrenamiento. El proceso se repite para cada valor de $\lambda$. El valor de $\lambda$ elegido será aquel que mejor resultado medio obtenga en términos de precisión de las cinco iteraciones

La Figura 4.5 ilustra el proceso de validación cruzada con diez iteraciones $(\mathrm{k}=10)$. En este proceso se toman 9 de los 10 conjuntos para entrenar el algoritmo y parte de ellas para verificar el resultado del entrenamiento. El proceso se repite para cada valor de $\lambda$. Una vez se han completado todas las iteraciones, el algoritmo de regularización tomará el valor de $\lambda$ que mejor resultado médio haya obtenido en términos de precisión de las cinco iteraciones

El algoritmo RLDA utiliza el parámetro de regularización durante el proceso de entrenamiento y validación. Del conjunto de datos totales obtenidos, el algoritmo RLDA utilizó un $90 \%$ del conjunto total para el entrenamiento del clasificador, mientras que como conjunto de test se tomó el $10 \%$ de los datos restantes. Partiendo de la precisión (DA) obtenida evaluando las muestras del conjunto de test correctamente clasificadas, se computa la información decodificada (DI) [26] cuantificando la cantidad de información sobre la dirección del movimiento contenida en las señales EEG extraídas en cada caso.

\subsubsection{Selección de electrodos}

Los algoritmos genéticos son métodos de búsqueda innovadores basados en fenómenos naturales, cuyas principales ventajas son su gran robustez y su independencia respecto del problema planteado. A lo largo de los últimos años, se ha venido de- 
Capítulo 4. Decodificación y clasificación de la señal EEG en entornos

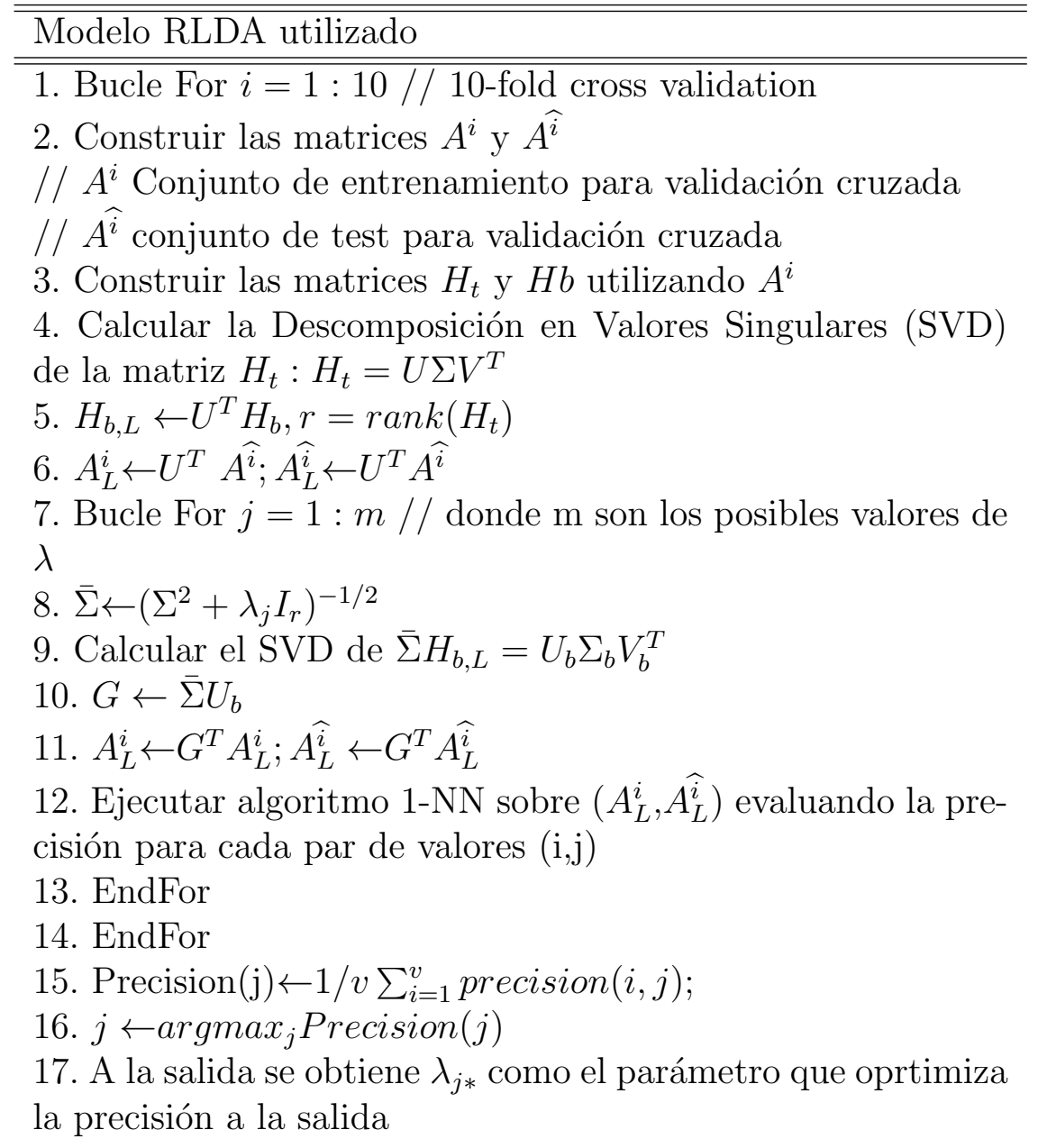

Cuadro 4.1: Modelo de aplicación del algoritmo RLDA utilizado para la clasificación de las muestras. Adaptado de [273]

mostrando la capacidad de los algoritmos genéticos en el ámbito de la optimización de parámetros [274].

Estudios recientes [275], han propuesto la utilización de algoritmos genéticos conjuntamente con SVM a través de un modelo híbrido, con el objetivo de llevar a cabo tareas de clasificación de señales EEG para evaluar su uso potencial en sistemas BCI. El uso del sistema BCI basado en señales EEG espontáneas requiere una clasificación precisa y el reconocimiento de múltiples canales EEG. El diseño de representaciones EEG y clasificadores para sistemas BCI es un tema sobre el que los investigadores trabajan actualmente, y cuya dificultad se encuentra en la extracción de complejos 
patrones espaciales y temporales de una serie temporal, ruidosa y multidimensional, obtenida de las medidas EEG. En este trabajo, se pretende demostrar que la combinación entre un algoritmo genético y el análisis del discriminante lineal regularizado puede maximizar la precisión (DA) de los sistemas BCI en entornos tridimensionales. Con el objetivo de incrementar la precisión (DA) durante el procedimiento de clasificación, el algoritmo genético seleccionará aquellos electrodos que mayor cantidad de información relevante contengan, relacionada con la tarea de movimiento ejecutada. Los electrodos irrelevantes no solo transportan información irrelevante o redundante, sino que también pueden contener señales ruidosas o erroneas que afectarían de forma importante al proceso generando resultados erróneos. En este sentido, los resultados demuestran que la selección de un subconjunto de características idóneas para el problema de clasificación incrementa la precisión del proceso utilizando datos nuevos, nunca antes analizados por el algoritmo. La selección de electrodos utilizando algoritmos genéticos se centra en clasificar las muestras tomando únicamente un subconjunto óptimo de electrodos. El objetivo principal de este paso es la selección de ese subconjunto óptimo de electrodos que permita maximizar la precisión (DA) al final del proceso de clasificación. Existe un amplio rango de posibilidades para identificar un subconjunto óptimo de electrodos con el objetivo de obtener la máxima precisión (DA). Aunque un subconjunto óptimo de electrodos podría ser obtenido mediante el uso de la fuerza bruta, el coste computacional del proceso de clasificación global sería demasiado alto. Por esta razón, el presente trabajo utiliza un algoritmo genético, debido a que es la mejor forma de seleccionar un subconjunto óptimo de electrodos con el mínimo coste computacional posible. Reduciendo el número de electrodos a tener en cuenta en el proceso de clasificación, se reduce de forma drástica el número de dimensiones de las muestras con la consecuente reducción del coste computacional. 


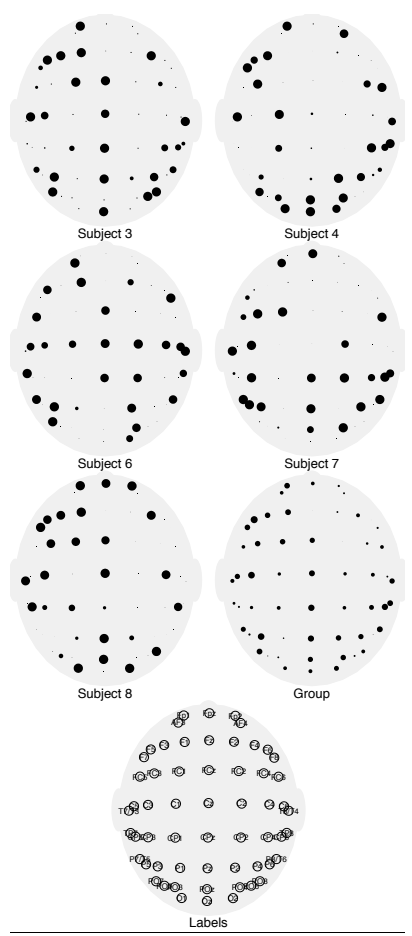

Figura 4.6: Selección de electrodos mediante algoritmo genético. El tamaño del círculo indica \% de relevancia de dicho electrodo ((El círculo de mayor tamaño indica un $100 \%$ de presencia, y una presencia del $0 \%$ está representada por un círculo vacío) para cada electrodo en el subconjunto óptimo de electrodos después de aplicar el algoritmo genético. Los electrodos que poseen circulos de mayor tamaño poseen información relevante que ha sido utilizada en mayor número de ocasiones que aquellos con círculos más pequeños

Debido al alto número de dimensiones de las muestras analizadas obtenidas por cada uno de los 58 electrodos activos que recogen información de la actividad EEG, resulta dificil obtener un valor óptimo en la precisión de la decodificación utilizando únicamente el algoritmo RLDA. Para proceder a la selección de los electrodos que aportan información útil en detrimento del resto se utilizará un algoritmo genético, que en combinación con el algoritmo LDA regularizado, permitirá obtener la máxima precisión en la decodificación para cada sujeto. Es importante destacar dos aspectos relevantes sobre la selección de electrodos mediante algoritmos genéticos. Por un lado, el algoritmo genético representa el conjunto de electrodos como un vector de 55 elementos binarios, donde el valor de cada elemento representa la relevancia o utilidad de cada electrodo. Por otro lado, la función de error está relacionada 
con la precisión obtenida por el algoritmo RLDA. El algoritmo genético controla la evolución del fenotipo con el objetivo de minimizar la función de coste. El error del clasificador será la función de coste que el algoritmo genético deberá minimizar. Antes de cada evaluación de los datos por parte del algoritmo RLDA y después de aplicar el algoritmo genético, los conjuntos de entrenamiento y test son utilizados por la función RLDA sin aquellos electrodos marcados con 0 como irrelevantes o ruidosos. La Figura 4.6 muestra los electrodos seleccionados tras la aplicación del algoritmo genético. El resultado obtenido para cada uno de los sujetos está representado en una imagen distinta. Los electrodos con mayor porcentaje de presencia en cada sujeto, han sido seleccionados mediante el algoritmo genético. Utilizando como muestras de entrada únicamente la información que transportan dichos electrodos y desechando la información procedente del resto, se ha conseguido el mejor resultado de DA de entre todas las posibles combinaciones de electrodos. La figura muestra los distintos conjuntos óptimos de electrodos seleccionados que han conseguido minimizar la función de error en la clasificación. En la Figura 4.6 se muestran las etiquetas de cada uno de los electrodos en una imagen separada. Puede apreciarse que los electrodos seleccionados en mayor número de ocasiones se corresponden con aquellos situados cerca de la corteza motora, del área premotora y de aquellas zonas relacionadas con el control del movimiento voluntario (Ver Capítulo 2).

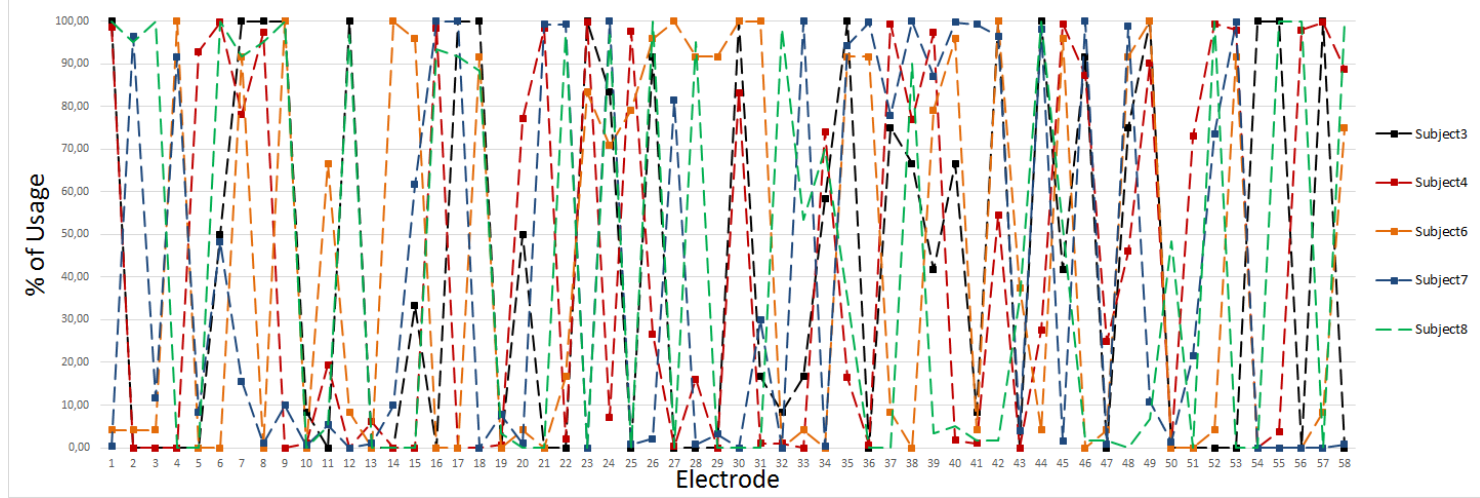

Figura 4.7: Porcentaje de presencia de cada uno de los electrodos en el subconjunto óptimo de electrodos seleccionado por el algoritmo genético. Cada sujeto posee un subconjunto óptimo de electrodos que optimiza la precisión en la decodificación. Cada una de las líneas de colores representa a un sujeto distinto 
El algoritmo genético evalúa combinaciones de electrodos con el objetivo de encontrar un conjunto de electrodos que permita minimizar la función de error. Para cada sujeto, el algoritmo trata de encontrar un subconjunto óptimo de electrodos que permita obtener un valor máximo de precisión (DA). El conjunto óptimo de electrodos que optimiza la precisión (DA) variará de un sujeto a otro. La Figura 4.7 muestra el porcentaje de presencia de cada uno de los electrodos en el subconjunto óptimo de electrodos seleccionado por el algoritmo genético en cada caso. En esta figura puede apreciarse que alguno de los electrodos (como es el caso de 7, 24, 26, 35, 39, 42, 44, 45, 48, 49, etc.) forman parte del subconjunto óptimo de electrodos prácticamente para todos los sujetos. Mientras que otros electrodos (como es el caso de 10, 11, 19, 20, etc.) no forman parte de ninguno de los subconjuntos de electrodos que optimizan la precisión en la decodificación.

\subsection{Resultados}

Los resultados obtenidos confirman las hipótesis expuestas en los primeros capítulos de esta tesis doctoral. La precisión obtenida durante el proceso de clasificación de las señales EEG evidencia que es posibloe obtener altas precisiones en sistemas BCI utilizando técnicas no invasivas. El análisis detallado de los resultados obtenidos en términos de precisión en la decodificación, así como su relación con la información decodificada, respaldan estas afirmaciones. Además, para evaluar el grado de mejora aportado por este estudio, al final de esa sección se realiza una comparativa de los resultados obtenidos con resultados de otros experimentos similares, analizando y comparando los valores de precisión (DA) e información decodificada (DI) obtenidos en un contexto similar. Los resultados de esta comparativa, muestran las bondades del modelo BCI desarrollado, arrojando unos valores significativamente mayores de DI y DA que el resto de estudios, incluso en el caso de experimentos basados en técnicas invasivas.

La Figura 4.8 muestra la precisión (DA) total obtenida tras la clasificación de las muestras de test y una vez entrenado el algoritmo. La precisión (DA) ha sido calculada tanto para cada individuo como para la media total de todos los sujetos en dominio del tiempo. Los vectores de características utilizados en cada caso represen- 
tan por un lado el fragmento inmediatamente anterior al inicio del movimiento o fase de planificación del movimiento (200ms antes del inicio del MO), y por otro lado el fragmento correspondiente a la fase de planificación del movimiento junto con la fase de movimiento desde el punto central hasta que el sujeto alcanza el objetivo (desde -200ms hasta 1,2ms alineado al MO). La precisión obtenida con cada uno de los dos conjuntos de datos y para cada uno de los sujetos puede observarse en la Figura 4.8.

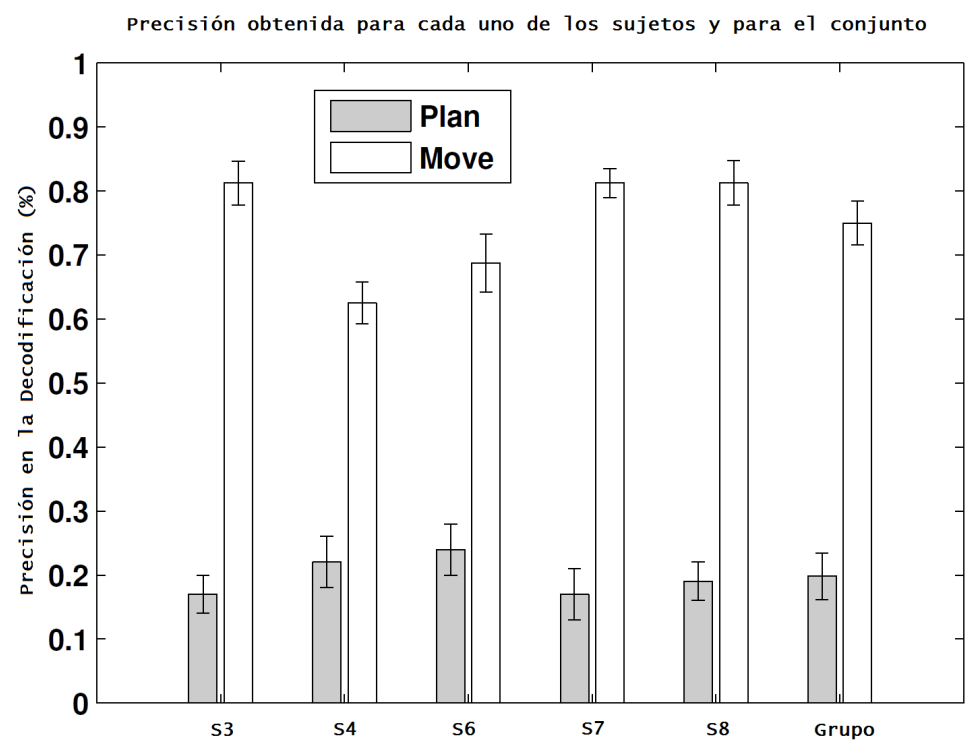

Figura 4.8: Precisión (DA) (\%) obtenida tanto para cada sujeto (S3, S5, S6, S7, S8) como para el conjunto de todos los sujetos $(\mathrm{N}=5)$ (Group). El nivel mínimo (chance) es $12.5 \%$ para ocho objetivos. 'Plan' corresponde al periodo de pre-movimiento ($200 \mathrm{~ms}$ - MO), mientras que en el caso de "Move" se ha utilizado la longitud completa del segmento de tiempo (-200 to $1.2 \mathrm{~s})$.

Evaluando el periodo justo anterior al inicio del movimiento, en la Figura 4.8 se desprende que la precisión media para todos los sujetos fue relativamente baja (21\%). Sin embargo, este porcentaje se encuentra por encima del nivel de oportunidad $(12.5 \%)$. Este valor es el resultado de dividir los resultados totales posibles (100\%) entre el número de clases posibles (8). Si el algoritmo arroja resultados significativamente superiores al $12.5 \%$, puede concluirse que las señales analizadas transportan información útil asociada a cada una de las direcciones del movimiento.

La Figura 4.8 muestra los resultados obtenidos por el clasificador para cada uno de los sujetos utilizando los datos EEG tanto del periodo de pre-movimiento como 
del periodo de movimiento del brazo hacia el objetivo final. La clasificación de los datos EEG asociados a la longitud completa del segmento de tiempo ha generado una precisión media del $75 \%$. Este valor ha sido obtenido teniendo en cuenta el conjunto de los sujetos analizados. Los mejores resultados tras la clasificación de las muestras de test han sido los asociados a los sujetos 3,7 y 8 con una precisión del $81.25 \%$. El peor resultado ha sido obtenido por el sujeto 4 con un valor de $62.5 \%$. Los resultados de precisión están significativamente por encima del nivel de oportunidad $(12.5 \%)$, lo que indica claramente que las señales EEG contienen información útil para decodificar la dirección del movimiento en el espacio tridimensional.

La evolución de la precisión en función del segmento decodificado también ha sido objeto de análisis. Para obtener la evolución de la precisión en función del tiempo, se han clasificado segmentos de distinta duración tomando el total de sujetos. Todos los intentos de los distintos usuarios tienen la misma duración. Partiendo únicamente del segmento que contiene la información recogida en los instantes anteriores al inicio del movimiento, se han ido añadiendo fragmentos de tiempo hasta llegar a la clasificación del segmento completo para el total de muestras. En la Figura 4.9 puede observarse la precisión (DA) como una función de la ventana de tiempo utilizada para la clasificación. La información sobre el movimiento ejecutado es transportada por tanto, en varias ventanas de tiempo utilizadas por el decodificador. La precisión (DA) se incrementa con el número de muestras utilizadas para la clasificación, aunque el periodo de pre-movimiento también contiene información sobre el "target"seleccionado según se desprende de los resultados de precisión obtenidos tras la evaluación del periodo de pre-movimiento.

La figura 4.11 muestra la matriz de confusión obtenida a partir las salidas del clasificador del conjunto de los sujetos. La estructura de la matriz de confusión permite entender cómo y dónde ocurren tanto los errores en la clasificación como las muestras clasificadas correctamente [276]. Una matriz de confusión permite visualizar el resultado del proceso de clasificación para algoritmos de aprendizaje supervisado. Cada columna de la matriz de confusión está asociada al número de predicciones de cada clase. Por otro lado, cada una de las filas de la matriz representan las clases a las que la muestra pertenece realmente. En este caso, las columnas indican la etiqueta real de la clase a la que pertenece la muestra, mientras que las columnas indican la 


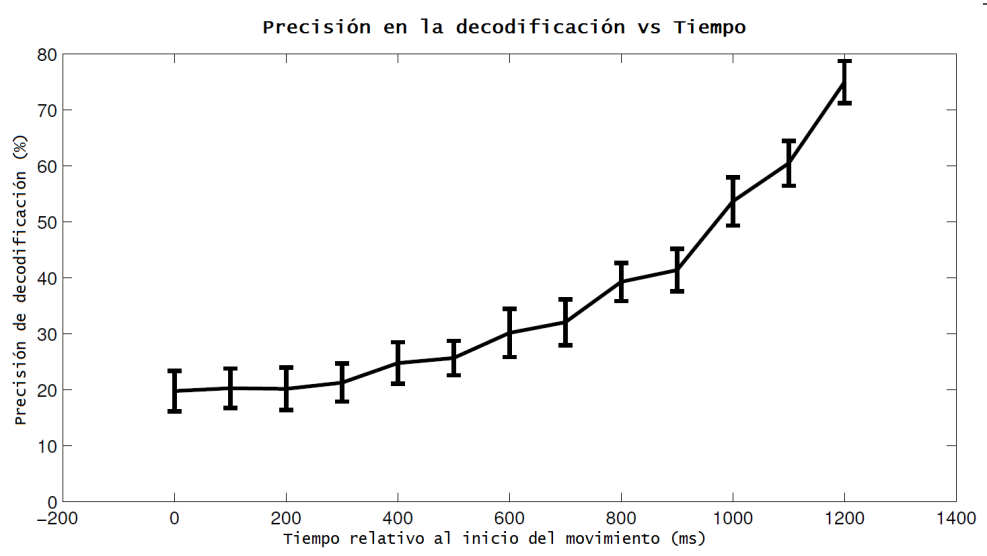

Figura 4.9: Evolución de la precisión (DA) utilizando señales EEG filtradas paso bajo en banda delta $(0.5-3 \mathrm{~Hz})$ como función de la longitud del fragmento evaluado (alineado con el inicio del movimiento (MO). El grafico muestra la precisión (DA) de diferentes fragmentos de tiempo: La precisión obtenida 200ms inmediatamente antes del movimiento esta indicado en la abcisa como tiempo 0 (MO). Los datos de precisión posteriores se obtienen tomando el fragmento total desde -200ms hasta el tiempo indicado en la abcisa, hasta tomar el fragmento completo desde -200ms hasta $1200 \mathrm{~ms}$. El nivel mínimo (chance) es $12.5 \%$ para 8 targets.

etiqueta asignada por el clasificador tras hacer la predicción. Así, cada celda indica la fracción de muestras de la clase indicada por la columna a la que pertenece, ha sido clasificada como perteneciente a la clase indicada por la columna en la que se encuentre dicha celda. Una muestra de datos EEG perteneciente a la dirección 4 ha sido correctamente clasificada si esta muestra se encuentra situada en el elemento $(4,4)$ de la matriz de confusión. En consecuencia, sólo las muestras que se encuentran situadas en la diagonal de la matriz de confusión han sido correctamente clasificadas. Estas muestras han sido asignadas por el clasificador a la clase a la que efectivamente pertenecían. La matriz de confusión (figura 4.11) también aporta información útil sobre las muestras clasificadas de forma errónea. Las muestras situadas en el elemento $(7,2)$ han sido clasificadas de forma errónea. Los datos EEG de esas muestras han sido obtenidos durante el desplazamiento del brazo del sujeto hasta el target número 7, sin embargo el clasificador ha asignado dichas muestras a la dirección 2. La matriz de confusión es una herramienta útil para comprobar el buen funcionamiento del clasificador o verificar si el sistema está confundiendo dos clases. En este caso, la matriz de confusión puede servir como herramienta para la evaluación de los 


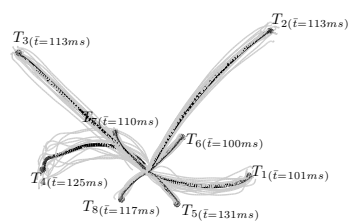

(a)

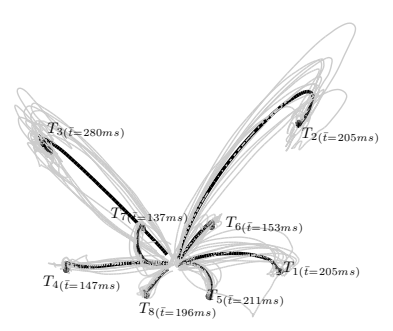

(d)

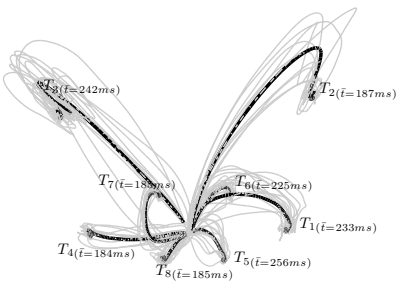

(b)

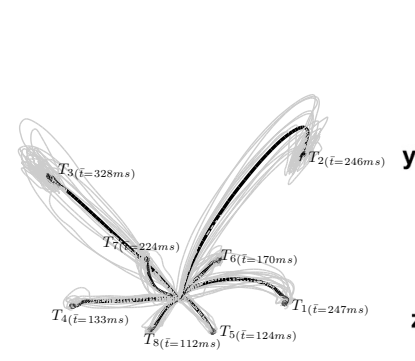

(e)

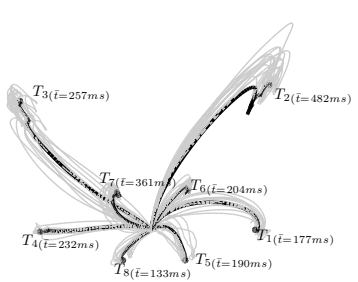

(c)

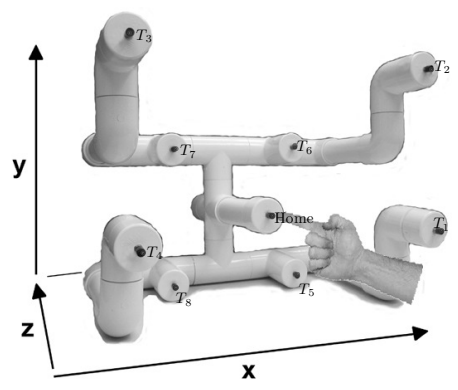

(f)

Figura 4.10: Configuración del experimento y trayectorias medias realizadas por cada uno de los sujetos. El artilugio utilizado para marcar el centro y los distintos objetivos (f) se muestra con el sistema de coordenadas cartesiano utilizado. Las trayectorias medias recorridas por el dedo de cada sujeto desde el centro al objetivo seleccionado (línea negra) y las trayectorias individuales para cada intento realizado (línea gris) se muestran en esta figura para cada sujeto. (a) Sujeto 3, (b) Sujeto 4, (c) Sujeto 6, (d) Sujeto 7, (e) Sujeto 8 
resultados obtenidos debido a que el número de muestras de entrada al clasificador es similar para cada una de las clases a clasificar.

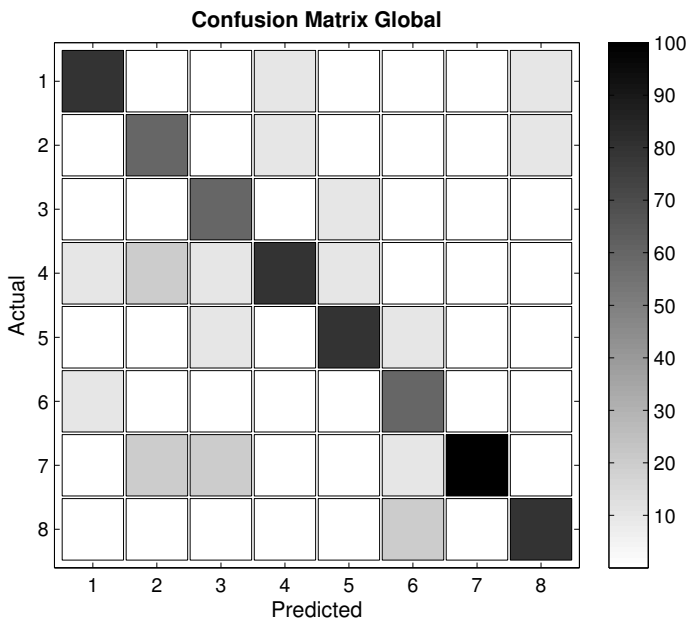

Figura 4.11: Matriz de confusión obtenida. Las celdas de color gris están relacionadas con la predicción realizada en cada caso (en \%). Cada etiqueta en . ${ }^{\mathrm{a}} \mathrm{ctual}$. etiqueta real de las muestras evaluadas por el clasificador, mientras que las etiquetas en.$^{\circ}$ utputrepresentan la clase predicha (etiqueta asignada) por el clasificador.

Una cámara colocada frente al sujeto registró las trayectorias del brazo del sujeto desde el centro hasta cada uno de los 8 objetivos en el espacio tridimensional (Figura 4.10f). La información detallada sobre la trayectoria hacia los distintos objetivos por parte de cada uno de los sujetos permite asegurar que las señales EEG captadas pertenecen a una trayectoria concreta. La Figura 4.10 Muestra las distintas trayectorias que han realizado los sujetos hasta cada uno de los targets en el espacio tridimensional. Las trayectorias medias recorridas por el brazo de cada sujeto se muestran en línea negra mientras que las trayectorias individuales de cada uno de los intentos se muestran en línea gris.

\subsection{Discusión y conclusiones}

Para evaluar si los resultados obtenidos por el sistema BCI suponen realmente un avance significativo, será necesario compararlos con otros estudios similares. Este movimiento puede ser decodificado a partir de las señales EEG en el mismo grado que ECoG. En este sentido, ECoG esta apoyado por estudios de decodificación 
Capítulo 4. Decodificación y clasificación de la señal EEG en entornos

\begin{tabular}{lll}
\hline \hline Autor & Técnica (No de sensores) / No de Targets & Precisión \\
\hline \hline Hammon 2008 [263] & EEG (140-150 Electrodos) / 3 Targets & $58.9 \%$ \\
Hammon 2008 [263] & EEG (140-150 Electrodos) / 4 Targets & $47.3 \%$ \\
Waldert 2008 [26] & EEG (20 Electrodos) / 4 Targets & $67 \%$ \\
Ball 2009 [277] & ECoG (112 Electrodos) / 8 Targets & $63 \%$ \\
Lew 2012 [278] & EEG (64 Electrodos) / 4 Targets & $92 \%$ \\
JS Woo 2015 [279] & EEG / 6 Targets & $74 \%$ \\
B Xia 2015 [280] & EEG (11 Electrodos) / 5 Targets (2D) & $96 \%$ \\
Y Li 2010 [44] & EEG (32 Electrodos) / 4 Targets & $90,75 \%$ \\
Este estudio & EEG (20 Electrodos) / 8 Targets & $75 \%$
\end{tabular}

Cuadro 4.2: Comparación entre los resultados arrojados por distintos estudios sobre la decodificación de la señal EEG durante el movimiento de la extremidad superior

basados en potenciales de campo locales de baja frecuencia, también llamados potenciales locales motores (LMP) o potenciales relativos a movimientos (MRP) [277] [14] [281]. Estas bajas frecuencias son probablemente las que menos atenuación sufrirán debido al cráneo [282] y podrían tener mayores relaciones de señal a ruido que señales localizadas en bandas de frecuencia más altas. De los resultados obtenidos en este estudio se desprende una conclusión clara: es viable decodificar las direcciones del movimiento del brazo en el espacio tridimensional mediante señales EEG. Los resultados demuestran que es posible obtener altas precisiones en la decodificación del movimiento utilizando amplitudes variables en el tiempo y aisladas $(0.5-3 \mathrm{~Hz})$ a partir de señales EEG obtenidas de forma no invasiva. La precisión (DA) obtenida es significativamente mayor que otros estudios similares que también han utilizado señales EEG. En este sentido, cabe destacar que la precisión obtenida en este estudio es comparable a la de aquellos estudios analizados que han utilizado tecnicas invasivas (Electrocorticograma ECoG) (Ver Tabla 4.2).

Los resultados del presente estudio muestran precisiones (DA) mayores que aquellos estudios realizados mediante tecnicas invasivas como ECoG [277], y en los que también se involucra al usuario para realizar experimentos con 4 y 8 objetivos espaciales que debe alcanzar para su posterior decodificación analizando las señales generadas. Con el objetivo de comparar los resultados expuestos anteriormente con otros estudios BCI sobre decodificación que han utilizado un número diferente de objetivos o una tecnica diferente de obtención de las señales cerebrales, se propone 


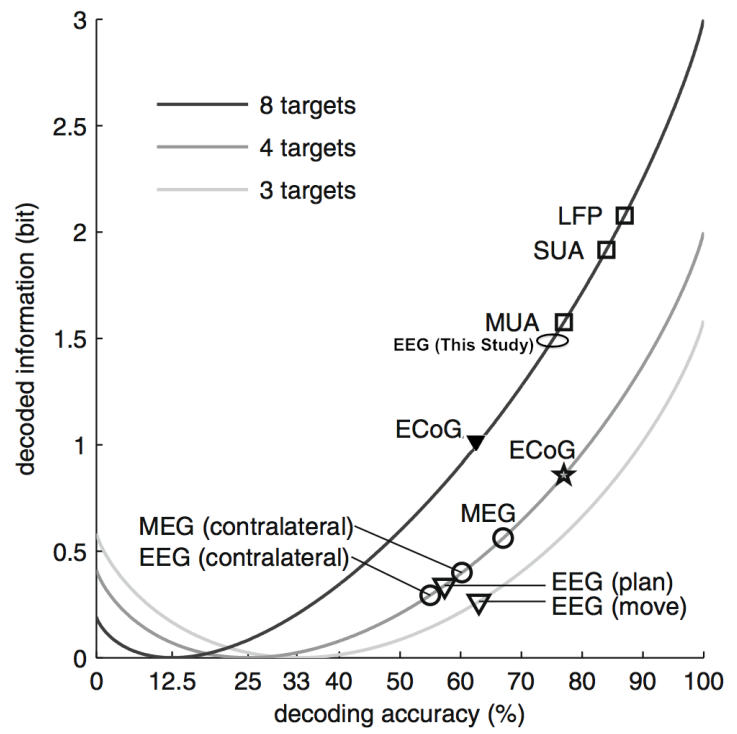

Figura 4.12: Comparación de diferentes tecnicas de obtención de señales cerebrales respecto de DA y DI relativas a la dirección del movimiento para tareas de desplazamiento de la mano desde un punto de referencia hasta cualquiera de los $N$ objetivos. La línea gris refleja la dependencia de DI sobre DA bajo ciertas premisas de simplificación (Ecuación 2 de [269]) para 3, 4 u 8 objetivos. Símbolos: Cuadrados abiertos — [283]; Estrella y triángulo sólido — [277]; circulos — [264] (En la referencia contralateral: Sólo fueron utilizados los sensores sobre el área motora contralateral); triángulos vacíos [263]; óvalo vacio - Este estudio (75\% DA). Adaptada y revisada, Figura 7 de [269]

la utilización del parámetro DI referenciado en secciones anteriores. Para cada uno de los experimentos se obtiene el valor de DI [26] a partir de DA (ecuación 4.1). Una vez obtenidos los resultados de DI, se incluyen los resultados obtenidos en el presente trabajo, para facilitar la comparativa, en la Figura 7 de [269] incluyendo estudios adicionales. La Figura 4.12 muestra la comparativa de la información decodificada (DI) para cada uno de los experimentos en función del número de targets y la precisión (DA) obtenida. Aquellos estudios que se han desarrollado en entornos con 3 o 4 objetivos y han arrojado una alta precisión pueden obtener valores menores en términos de información decodificada que otros con precisión similar pero cuyo experimento se ha desarrollado en un entorno de 8 targets. Es evidente que las señales deben disponer de mayor cantidad de información espacial para diferenciar entre 8 
clases en un entorno tridimensional que para poder clasificar muestras en entornos de dos dimensiones y 4 clases. La simple comparación de la precisión obtenida en cada uno de los estudios no es suficiente para verificar que se ha realizado un avance importante respecto de otros estudios similares. El parámetro DI permite comparar los distintos estudios de decodificación del movimiento a pesar de que cada uno de ellos se desarrolle en entornos con distinto número de targets. En este caso, los resultados obtenidos por el presente estudio en términos de información decodificada (DI), avalan las bondades del sistema BCI diseñado.

Tal y como se desprende de los resultados presentados en este trabajo, y apoyado por recientes estudios [268], es posible concluir que los sujetos pueden aprender a utilizar la actividad EEG para controlar el movimiento tridimensional. La mayoría de estudios basados en grabaciones análogas (LFP o ECOG) se han centrado generalmente en la identificación de la banda o bandas de frecuencia óptima para la decodificación basada en la potencia espectral [277] [284] [23] [22]; asumiendo que la resolución espacial de las frecuencias en banda gamma son críticas para la decodificación del movimiento. Sin embargo, algunos de los estudios anteriormente citados han evaluado la posibilidad de realizar la decodificación a partir de potenciales relativos al movimiento (en dominio del tiempo) asi como utilizando las modulaciones espectrales de amplitud (en dominio de la frecuencia) en muy bajas frecuencias y en la banda gamma alta. Especificamente, en [277] se reportó una alta precisión en la decodificación de la dirección del movimiento del brazo basada en la modulación de amplitud tanto en la banda delta $(<2 \mathrm{~Hz})$ como en la banda gamma alta (52-128 $\mathrm{Hz}$ ), obtieniéndose valores de precisión considerablemente más altos en comparación con las bandas alpha, beta y banda gamma baja. Otros estudios recientes [285] han desarrollado modelos BCI similares para el control de un brazo robótico antropomorfo en el espacio tridimensional. Sin embargo, la señal cerebral ha sido obtenida de forma invasiva a través de 96 arrays de microelectrodos, restando versatilidad y aplicabilidad al sistema fuera del laboratorio.

Desde que algunos autores [286] examinaron la decodificación de la dirección del movimiento hacia unos objetivos determinados basados en la actividad individual de una unidad (SUA) y señales LFP, concluyeron que las precisiones decodificadas de la grabación simultánea de señales SUA y LFP son muy similares. Mientras que la 
información direccional varía con la frecuencia de LFP, siendo mayor en la banda de frecuencias baja $(0.3-4 \mathrm{~Hz})$ y alta $(48-200 \mathrm{~Hz})$ que en la bandas de frecuencia intermedias. Ellos atribuyen la mayor precisión obtenida a la organización espacial de las señales LFP sobre las áreas de grabación (cortezas primaria motora y premotora). Ademas, algunos autores [287] mostraron la posibilidad de decodificar el movimiento de alcance y agarre en tres dimensiones a partir de señales LFP basadas en información de las bandas delta y gamma. Teniendo todo esto en cuenta, los estudios EEG, ECoG y LFP anteriormente mencionados y los resultados mostrados en el presente trabajo indican que la información sobre la dirección del movimiento esta contenida tanto en la banda delta como en las frecuencias altas de la banda gamma. En este sentido, las señales cerebrales obtenidas mediante tecnicas no invasivas como EEG, pueden ser una fuente de señal conveniente para la decodificación de la intención del movimiento en aplicaciones BCI 
Capítulo 4. Decodificación y clasificación de la señal EEG en entornos 


\section{Capítulo 5}

\section{Conclusiones}

El verdadero progreso es aquel que pone la tecnología al alcance de todos

Henry Ford

\subsection{Logros}

El sistema BCI desarrollado en la presente tesis doctoral, utiliza técnicas de selección de caracteristicas y algoritmos de clasificación de la señal EEG. A la vista de los resultados obtenidos, ha quedado demostrada la viabilidad de decodificar la intencionalidad del sujeto con una precisión comparable a la obtenida por otros estudios que han utilizado sistemas invasivos. En este sentido, ha quedado demostrado que es posible obtener altas precisiones en la decodificación de la señal electroencefalográfica en sistemas BCI. Se han refrendado las teorías desarrolladas respecto de la señal EEG y las posibilidades futuras del BCI con experimentos que han certificado su validez. Concretamente, este trabajo demuestra la viabilidad de decodificar las direcciones del movimiento de una extremidad superior hacia ocho objetivos dispuestos en el espacio tridimensional. Para el desarrollo de este trabajo se han utilizando amplitudes variables en el tiempo a partir de señales EEG obtenidas de forma no invasiva. La precisión obtenida (DA) puede ser comparada tanto con aquellos estudios analizados que han utilizado técnicas invasivas (ECoG) como con aquellos que han utilizado técnicas no invasivas o EEG (Ver Capítulo 4). Sorprendentemente, los 
resultados del presente trabajo muestran precisiones mayores que aquellos estudios realizados mediante técnicas invasivas como ECoG [277], y en los que también se involucra al usuario para realizar experimentos con 4 y 8 objetivos espaciales que debe alcanzar. Es evidente que este trabajo, realizado con técnicas no invasivas, ha obtenido mejores resultados en términos de precisión que el experimento con técnicas invasivas anteriormente mencionado. Todo ello, a pesar de que tal y como se comentó anteriormente, [277] ha utilizado el doble de electrodos, técnicas invasivas, una relación señal a ruido mucho mayor y un mismo número de targets distribuidos en el entorno tridimensional. A lo largo de la presente tesis doctoral se han descrito distintos casos en los que los sistemas BCI han sido utilizados para controlar dispositivos externos a través de señales cerebrales. A la vista de los sistemas expuestos en los primeros capítulos, se aprecia un avance importante tras analizar y comparar los resultados obtenidos con el modelo BCI no invasivo propuesto en el presente trabajo. Evaluando la amplitud de la señal EEG y utilizando algoritmos genéticos para la selección de características, se han obtenido altas precisiones en la decodificación de la señal. El método de selección de características ha desechado aquellos canales que no transportaban información útil, utilizando para la clasificación solo aquellos que contenían información relevante respecto de la tarea ejecutada. Los electrodos localizados en zonas de la corteza cerebral con funciones relacionadas con el movimiento voluntario, han sido los mayoritariamente seleccionados por el algoritmo para utilizar la señal EEG captada como entrada al algoritmo de clasificación. Este hecho, junto con las altas tasas de precisión en la decodificación, demuestran que la señal EEG codifica información relevante respecto de procesos cerebrales relacionados con el movimiento voluntario.

Aumentar la precisión de los sistemas BCI no invasivos abre un amplio abanico de posibilidades para desarrollar dispositivos robóticos de asistencia al movimiento. Se planteó el desarrollo de un nuevo modelo de BCI que permita añadir robustez y nuevas funcionalidades al modelo propuesto como uno de los objetivos del presente trabajo. Permitiendo la interacción directa entre GoogleGlass y un dispositivo EEG para controlar una prótesis robótica, se abren nuevas líneas para el desarrollo de sistemas robóticos controlados por el usuario a través de las señales cerebrales. Esta interacción permitirá que los sistemas BCI puedan salir de los laboratorios de inves- 
tigación para ser utilizados en otro tipo de entornos. La conexión entre GoogleGlass y un dispositivo EEG, junto con el modelo BCI desarrollado en el presente trabajo, permitirá aumentar las formas de interacción del sujeto con su entorno. Todo ello, combinado con un modelo BCI no invasivo con altas precisiones de decodificación en entornos tridimensionales, permitirá eliminar algunas de las barreras que hasta ahora han impedido un mayor desarrollo de las prótesis robóticas BCI.

\subsection{Desarrollos futuros}

Algunos autores han propuesto en el pasado esquemas para el control de acciones en un robot [288] mediante algoritmos de inteligencia artificial en combinación con información visual. Estos sistemas han estado centrados en el control de la percepción visual, la percepción táctil y la interacción motora, implementando modelos biológicos en distintos subsistemas. El desarrollo que integra un monitorizador BCI junto con las GoogleGlass, abre una importante línea futura que, integrada con el modelo del estudio anterior, puede generar importantes avances en este campo. Este modelo se sirve de tres elementos fundamentales para interaccionar con el entorno y recabar información: un cabezal para la entrada de datos visuales, un brazo robótico y un sistema táctil. La aplicación del modelo desarrollado en esta tesis doctoral, junto con la implementación del modelo de comunicación entre las GoogleGlass y un sistema BCI portátil, abre la puerta a la simplificación y mejora del modelo propuesto en [288].

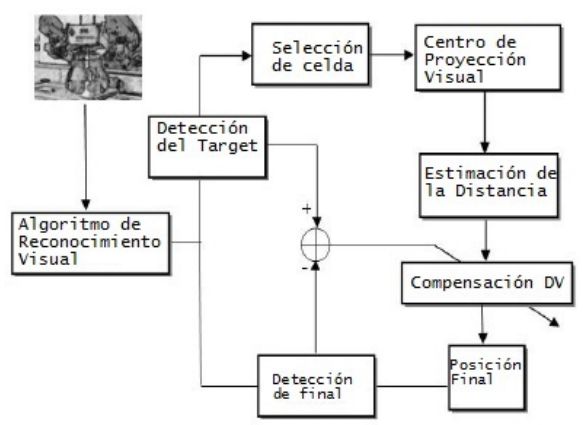

Figura 5.1: Esquema general de funcionamiento del sistema [288] 
La operación del sistema se realiza en dos fases: En primer lugar se ejecuta una fase de aprendizaje en la que se calculan los pesos de la red neuronal que realizarán la transformación entre lo que la máquina percibe (Información sensorial procedente de la visión por computador y el sensor de presión tactil) y la posición a la que se debe llevar el brazo robótico. Es lógico que el desplazamiento del brazo robótico sea una función de las observaciones del sistema, de ahí que sea un algoritmo no supervisado, ya que no se dispone de información a priori sobre una función densidad de probabilidad acerca de una tarea concreta a realizar por el brazo, todo se basa en las observaciones. Por tanto, para traducir la información sensorial a movimientos o posiciones del brazo es necesaria una matriz intermedia de pesos "Z" que es objeto de calibración y cálculo en esta primera fase del aprendizaje. El cálculo de la matriz de pesos se realiza mediante el algoritmo LMS (Least Mean Square) ya que la principal funcionalidad de este algoritmo es el encontrar los coeficientes que permiten obtener el valor esperado mínimo del cuadrado de la señal de error, definida como la diferencia entre la señal deseada y la señal producida a la salida. Posteriormente tiene lugar la fase de operación, en la que se ejecutan las tareas propias del sistema en base a lo ocurrido en la primera, teniendo en cuenta otros condicionantes externos. En esta fase debe existir una retroalimentación que esté continuamente evaluando el "vector diferencia" entre la posición actual del brazo y la posición deseada, con la ventaja adicional de poder demandar una nueva posición del brazo una vez el movimiento hacia un punto deseado ha comenzado. Esto permite que no se tenga que esperar a que el brazo llegue al punto demandado para pedir el cambio en la trayectoria, lo cual añade una prestación importante que confirma la robustez a priori del sistema. En contra de lo que ocurre en otros sistemas, en la fase de operación es posible también que los pesos cambien o evolucionen su valor. Tras el proceso de aprendizaje y ya en la fase de operación es posible hacer que el sistema aprenda nuevas maniobras utilizando un sencillo sistema cuya filosofía es similar al algoritmo de descenso por gradiente. Se utiliza un factor $\mu$ como parámetro de aprendizaje, en función del cual el algoritmo se desliza por la superficie de error, hasta obtener los valores óptimos de los pesos que hacen el error mínimo en una determinada situación. Las coordenadas motoras se obtienen a través de una transformación multiplicando los pesos de la red neuronal por las coordenadas espaciales, por tanto será posible evaluar el error 
producido entre estos dos valores que debieran ser iguales en condiciones ideales. La situación de mínimo error nos dará el valor de los pesos óptimos.

La Figura 5.1 muestra el esquema general de funcionamiento del sistema de control desarrollado por [288]. En los últimos años la comunidad científica ha trabajado intensamente para mejorar el diseño y prestaciones de los brazos robóticos antropomorfos [289] [290] [291]. La interacción entre el modelo BCI tridimensional y no invasivo propuesto en esta tesis, el esquema descrito en la Figura 5.1 y los últimos avances en el ámbito de la robótica, permiten abrir esta línea de desarrollo, combinando los útimos progresos de cada campo. En el presente capítulo, se propone el desarrollo de un sistema que posibilite aumentar notablemente las prestaciones de los sistemas BCI actuales. Introduciendo la posibilidad de su utilización fuera del laboratorio.

\subsection{Nuevo sistema basado en GoogleGlass y BCI}

La introducción de innovaciones tecnológicas en campos como la medicina esta propiciando mejoras que permiten mejorar la eficacia y eficiencia de los procedimientos médicos. Desde el punto de vista asistencial, el desarrollo de la telemedicina está cambiando la atención médica, el diagnóstico y el seguimiento de personas mayores, dependientes y enfermos. La telemedicina puede ser definida como el uso de las tecnologías asociadas a las telecomunicaciones con el objetivo de suministrar información y servicios médicos. Aunque esta definición pueda estar fundamentalmente asociada al uso de tecnologías asociadas al teléfono, la telemedicina está siendo usada cada vez más como un elemento remoto para la consulta clínica. Los gobiernos están utilizando recursos propios para construir el estado del arte de los sistemas de telemedicina, en algunos casos con inversiones superiores a los 50 millones de dólares [292]. Las innovaciones desarrolladas en forma de dispositivos, elementos robóticos, algoritmos, protocolos de comunicación, etc. son incorporadas al campo de la medicina como herramientas valiosas con las que aumentar la eficacia y eficiencia de los tratamientos médicos. Con el desarrollo de la neurorehabilitación, las estrategias de rehabilitación físicas para las extremidades superiores están siendo aceptadas gradualmente por terapeutas e investigadores del campo de la medicina. 
Estas estrategias incluyen intervenciones intensivas, entrenamiento orientado a tareas y entrenamiento bilateral. Recientemente [293] ha sido posible desarrollar un dispositivo exo esqueleto para rehabilitación de las extremidades superiores que puede ser utilizado para entrenamiento bilateral. Este dispositivo ha sido evaluado por terapeutas experimentados durante el diseño del proceso para determinar su potencial en aplicaciones clínicas. Los resultados experimentales indican que la resolución del movimiento es efectiva y que este tipo de movimiento bilateral puede ser implementado utilizando el dispositivo exo esqueleto desarrollado [293]. Algunos autores [294], sugieren que después de ciertas intervenciones, la mayoría de los pacientes que han sufrido derrames cerebrales quedan con una gran limitación en las caracteristicas funcionales de la mano. La robótica para rehabilitación ha demostrado ser una herramienta útil a la hora de mejorar el proceso de rehabilitación y acelerar la recuperación funcional de la mano. Debido al papel crucial que juega la mano en las actividades cotidianas de la vida, las terapias relacionadas con la recuperación funcional de este miembro están incrementándose recientemente. Los resultados obtenidos demuestran que la aplicación de tecnología para complementar las labores de rehabilitación aceleran el proceso de rehabilitación y mejoran la precisión de las trayectorias que realiza el sujeto, permitiendo al sujeto extender y flexionar sus dedos libremente [294]. Los ejemplos descritos, muestran el abanico de posibilidades generadas cuando los últimos avances tecnológicos se introducen en campos como la medicina quirúrjica, la restitución del movimiento o las neuroprótesis. En este campo, también se ha incorporado tecnología en el campo de la monitorización de las señales vitales para facilitar el seguimiento, el control y el diagnóstico de los pacientes. Durante los últimos años se han dedicado muchos esfuerzos desde la comunidad científica para el diseño y desarollo de sistemas basados en biosensores que el sujeto puede llevar puesto a todas horas. Todo ello ha estado motivado por el incremento de los costes del sistema de salud y por los avances tecnológicos en el campo de la miniaturización de los biosensores, las prendas inteligentes, la microelectronica y las comunicaciones inalámbricas. Esta tecnologia puede facilitar el desarrollo de dispositivos de bajo coste y no invasivos para la monitorización del estado de salud y la actividad mental del sujeto en cualquier lugar y en cualquier momento. Recientes estudios [? ] han analizado el estado de las investigaciones de los sistemas de bio- 
sensores para la monitorización de la salud con el objetivo de evaluar el nivel de madurez de las investigaciones realizadas en los últimos años en este campo. Todos estos estudios, apuntan hacia un futuro en el que la tecnologia formara parte de la vida cotidiana del sujeto, permitiendo no solo la monitorización continua de su estado de salud, sino también facilitando la interacción del sujeto con su entorno a traves de tecnologia que se lleva puesta. En el futuro, este tipo de sistemas deberán estar basados en la facilidad de uso, la capacidad para su integración plena en la vida cotidiana y una gran versatilidad.

\subsubsection{Actuadores en sistemas BCI}

El campo del Brain Computer Interface también puede verse afectado positivamente por estos desarrollos tecnológicos. La irrupción de nuevos dispositivos y componentes electrónicos que pueden ser utilizados por un sistema BCI para captar la señal cerebral, clasificarla, recoger información de su entorno o para interaccionar con él. Esto puede mejorar la funcionalidad de estos sistemas abriendo nuevas vias de desarrollo. Llegado este punto, es posible plantearse la posibilidad de combinar el desarrollo tecnológico descrito anteriormente junto con el modelo BCI desarrollado en capítulos anteriores. A lo largo del presente capítulo, se propone el desarrollo de un sistema BCI para monitorización de la actividad cerebral e interacción con el entorno. A partir de dispositivos desarrollados en los últimos años, se ha diseñado un modelo de BCI versátil que el sujeto puede llevar puesto en cualquier lugar y situación.

El desarrollo principal de esta tesis doctoral permite a un sujeto seleccionar un objeto de entre ocho posibles, situados en un entorno tridimensional. Gracias al desarrollo propuesto, ha sido posible demostrar que, a traves de señales no invasivas, los sujetos pueden llevar a cabo esa selección con una precisión muy alta, a través de un dispositivo BCI. Los experimentos desarrollados por sistemas BCI han estado contextualizados de forma general en un entorno aislado en los laboratorios de investigación [295] [296] [39]. Sin embargo, cada vez más investigadores trabajan para tratar de eliminar las limitaciones que impiden a los sistemas BCI salir del laboratorio para ser incorporados plenamente a la vida cotidiana. La primera de las limitaciones está relacionada con la utilización de electrodos húmedos tradicionales que acarrean 


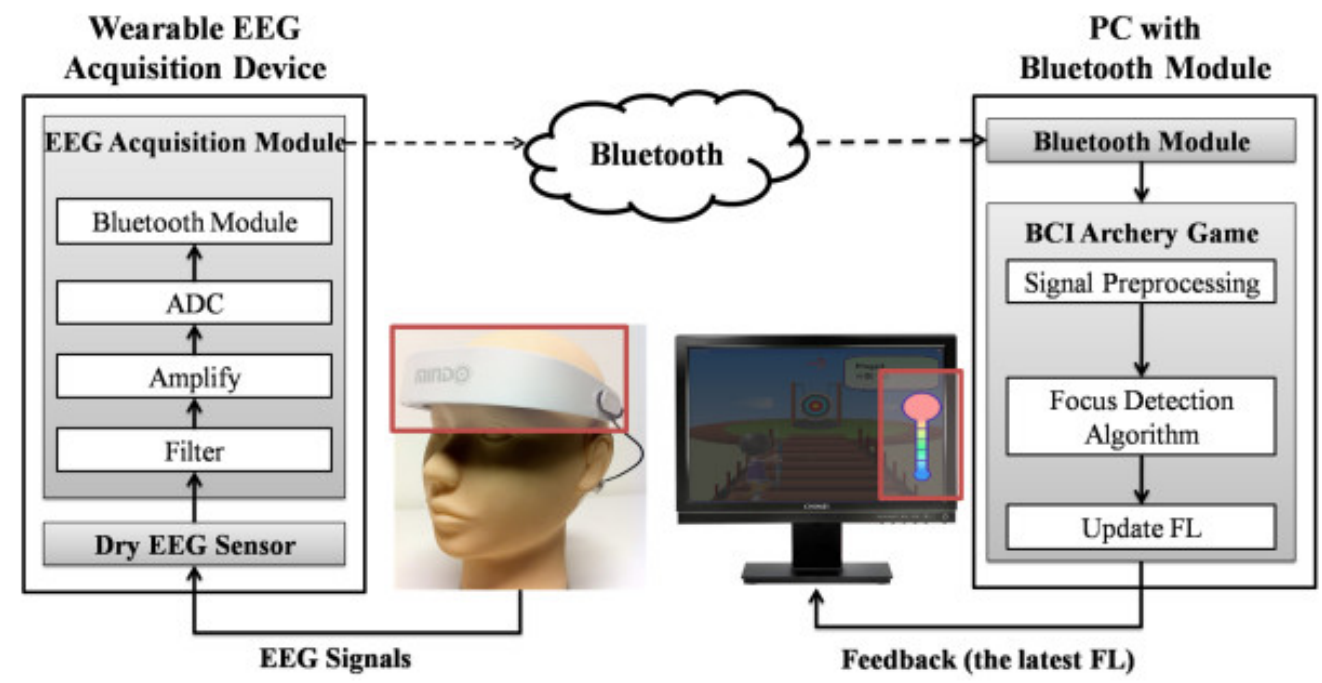

Figura 5.2: Esquema del sistema BCI autónomo con electrodo seco para control de un videojuego de forma inalámbrica [297]

desventajas importantes y no sólo requieren gel conductor, sino también una cierta preparación previa en la piel del usuario. Para abandonar los laboratorios es fundamental disponer de sistemas BCI que incorporen tecnología inalámbrica, permitiendo analizar y procesar la señal captada eliminando las conexiones físicas. Recientes estudios [297] proponen un sistema BCI inalámbrico Figura 5.2 que permite controlar un videojuego utilizando electrodos secos. Este sistema ha demostrado buenas prestaciones en la detección de estados cognitivos en tiempo real y su aplicación al control BCI. Los buenos resultados arrojados por este estudio confirman la viabilidad de utilizar de este tipo de dispositivos BCI para interaccionar con el exterior.

Si la incorporación de hardware puede aumentar considerablemente las prestaciones de los sistemas BCI, la introducción de nuevos desarrollos software relacionados con la realidad aumentada, puede suponer una auténtica revolución en la forma de operar los sistemas BCI del futuro. Se han dado pasos importantes recientemente [298], introduciendo un paradigma novedoso de realidad aumentada llamado "mindmirror". Este sistema permite visualizar lo que está ocurriendo en el cerebro del sujeto en tiempo real. La actividad cerebral es extraída y analizada en tiempo real mientras que el sujeto puede ver un cerebro virtual en una pantalla colocada frente a él. Esto permite que el usuario pueda aprender progresivamente cómo controlar los 
diferentes estados mentales, gracias a la información que se muestra a través de la realidad aumentada. Estos desarrollos no sólo pueden ser interesantes desde el punto de vista neurológico, sino que también podrían ser aprovechados en campos como la educación o el entretenimiento.

A pesar de que en los ejemplos descritos anteriormente se avanza en la autonomía de los sistemas BCI, sigue siendo necesario disponer de una computadora con la que el sistema BCI pueda interactuar. La incorporación de dispositivos inteligentes, realidad aumentada, 'wearablesó tabletas en combinación con el elemento de monitorización BCI puede aumentar las prestaciones y autonomía de los sistemas. La mejora conjunta de estos elementos, generará nuevos sistemas BCI más robustos, más versátiles y con mejores prestaciones.

La tecnologia BCI posee evidentes limitaciones para su uso generalizado por parte del gran público. Los sistemas EEG actuales necesitan un tiempo importante para su colocación y calibración. Con el paso de los años, las distintas compañías han ido desarrollado productos hardware y software que permiten monitorizar y tratar la señal EEG de forma portatil e inalámbrica como g.MOBIlab+ [299], Emotiv EPOC [300] o Neurosky MindWAVE [301] . Estos desarrollos constituyen la base de los sistemas BCI del futuro, incorporando los avances en este campo con los nuevos dispositivos robóticos de última generación.

La introducción de sistemas auxiliares a los dipositivos BCI existentes permitirán dotarlos de nuevas funcionalidades, aumentando sus prestaciones de forma exponencial. Hace algunos años que los sistemas BCI son utilizados para interactuar con videojuegos. Los desarrolladores comenzaron por juegos sencillos como el que se ilustra en la Figura 5.3. Es evidente que esta nueva forma de utilización de los sistemas BCI abrió una nueva forma de interactuar con el exterior utilizando las señales EEG del usuario. La comunidad cientifica ha ido perfeccionando este tipo de interacciones dentro de la industria del videojuego. Uno de los últimos logros puede apreciarse en la Figura 5.4, en la que un sujeto controla un videojuego de cierta complejidad como Warcraft a través de un sistema BCI.

Los dispositivos BCI también han sido utilizados como actuadores dentro del campo de la telefonía móvil. La Figura 5.5 muestra un ejemplo de las posibilidades que la introducción de la tecnologia BCI puede ofrecer en la vida diaria a personas con 


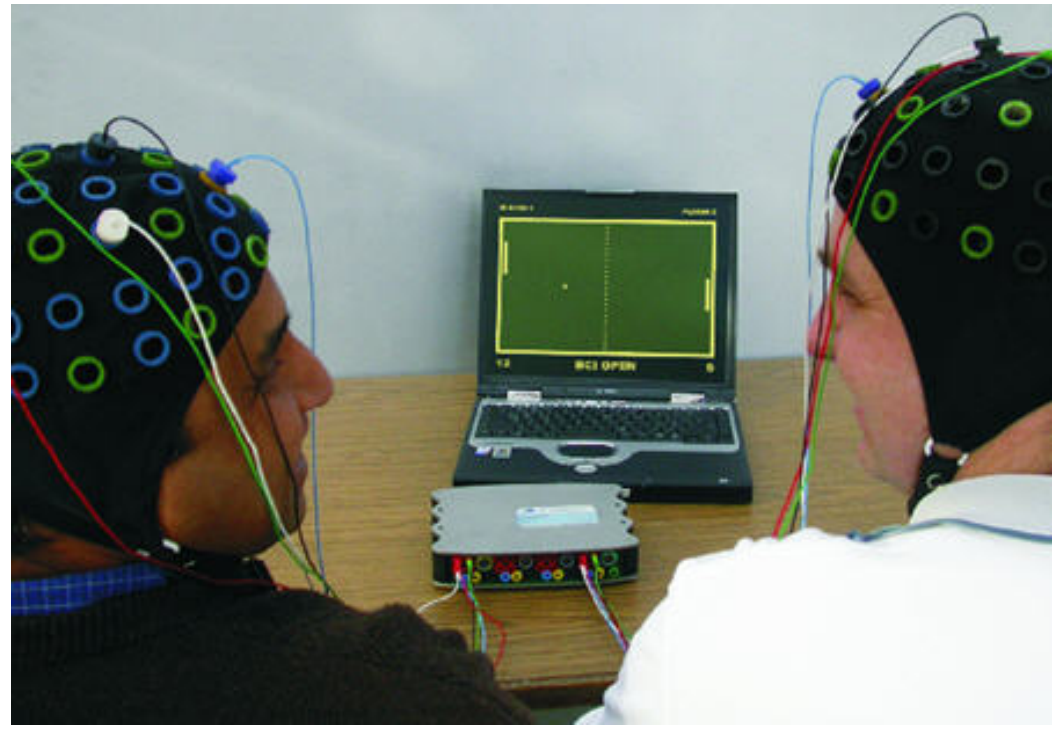

Figura 5.3: Ejemplo de uso de un sistema BCI interactuando con el videojuego PingPong en la versión de dos usuarios

lesiones motoras. Los autores [302] de este estudio proponen el uso de señales EEG para controlar un teléfono móvil sin esfuerzo, prescindiendo de usar las manos y de forma silenciosa. Tomando como base los principios de los sistemas BCI que utilizan el paradigma P300, el dispositivo telefónico va mostrando una secuencia de fotografias que corresponden a los contactos almacenados en el teléfono. El sujeto selecciona el contacto deseado a traves de la activación del potencial P300. La actividad cerebral es captada por el dispositivo EPOC Emotiv y se transmite por tecnología inalámbrica al teléfono donde se ejecuta una aplicación nativa que discrimina las señales P300 del ruido. Actualmente, la comunidad científica trabaja para mejorar estos prototipos y hacerlos más practicos, robustos y versátiles.

En los últimos años los vehículos aéreos no tripulados están experimentando un interés creciente por parte de muchos sectores. Los drones están siendo utilizados en diversos campos con fines muy distintos, desde la captación de fotografias aéreas hasta el análisis de terrenos cultivados, pasando por aplicaciones militares y de reconocimiento. Un dron puede ser utilizado también como un dispositivo a controlar por un sistema BCI (ver Figura 5.6).

Hay casos en los que el usuario no está completamente impedido y necesita rehabilitación para restituir el movimiento en ciertas zonas del cuerpo. Tradicionalmente 


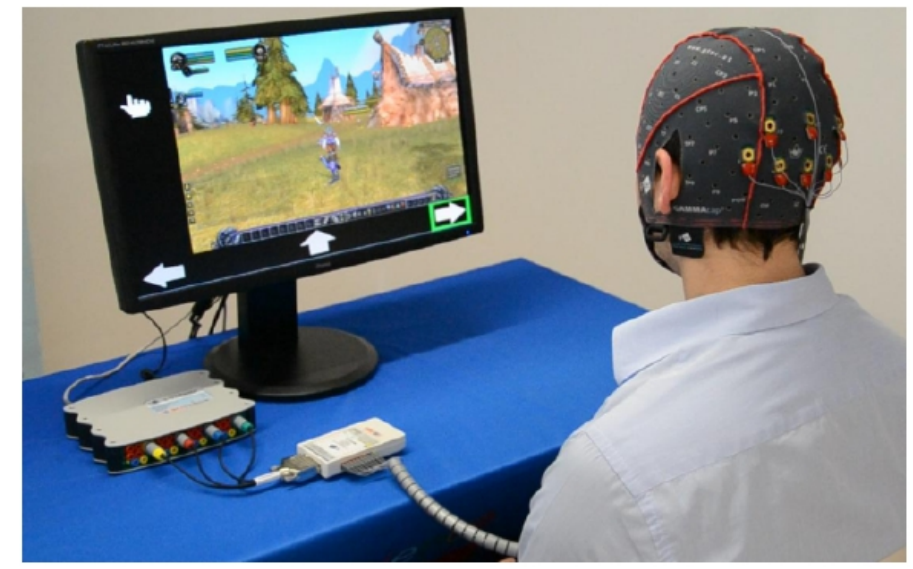

Figura 5.4: Ejemplo de uso de un sistema BCI para controlar la acción durante la ejecución del videojuego Warcarft

la rehabilitación se ha llevado a cabo en clínicas de fisioterapia con personal especializado. Sin embargo, existe la posibilidad de llevar a cabo la terapia de rehabilitación asistida a través de un dispositivo BCI. Un elemento robótico fijado adecuadamente a la zona concreta a rehabilitar permite al sujeto controlar la rehabilitación a través de sus ondas cerebrales. La Figura 5.7 ilustra la configuración del sistema BCI que permite el movimiento asistido de ciertos gestos de la mano.

También existe la posibilidad de controlar otros elementos de mayor embergadura a través de sistemas BCI. Es el caso del sistema de la Figura 5.8 en el que un sujeto utiliza el sistema BCI para controlar el desplazamiento de una silla de ruedas. Tal y como se comentó en el Capítulo 1, este sistema resulta especialmente útil para pacientes con lesiones físicas importantes que tienen en los sistemas BCI la única alternativa posible para controlar sus movimientos.

En el cine es posible encontrar multitud de ejemplos en los que se aborda, de forma directa o indirecta, la utilización de sistemas BCI con distintos objetivos. La comunidad cientifica ha realizado experimentos orientados al control de cuerpos completos a través del cerebro de un sujeto. La Figura 5.9a ilustra un sistema en el que a través de las señales cerebrales, el usuario es capaz de controlar una serie de funciones de un robot antropomorfo completo como si fuera su propio cuerpo. La película Ávatar ilustra un caso evidente de la utilización por parte del cine de estos sistemas. A través de una cama de control como la de la Figura 5.9b, el cerebro 


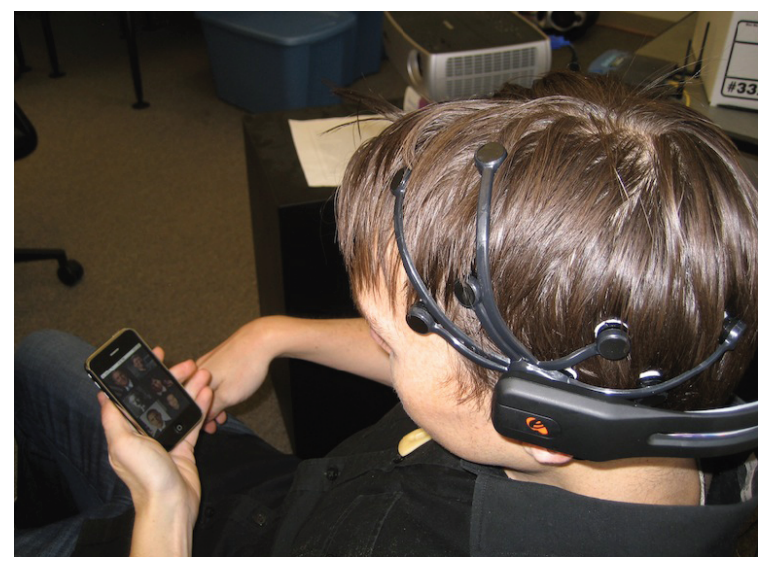

Figura 5.5: Un sujeto haciendo uso del sistema Neurophone a traves de EPOC Emotiv [302]

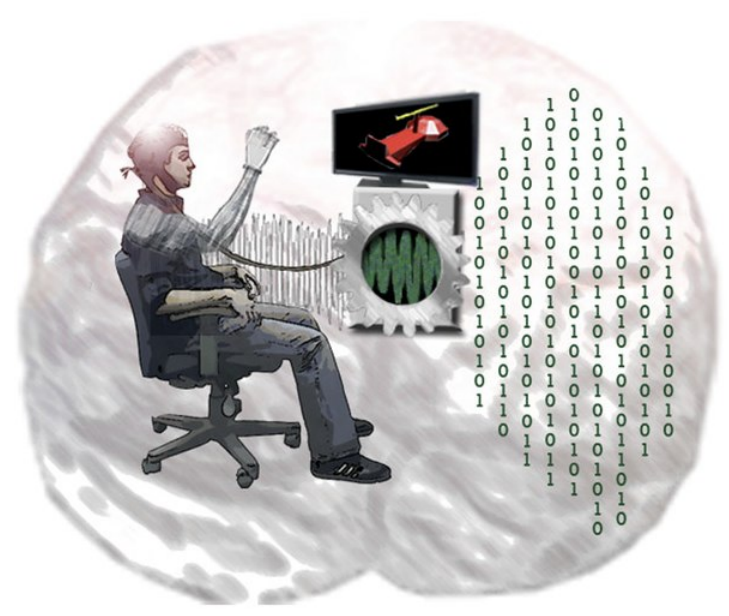

Figura 5.6: Sistema BCI utilizado para controlar vehículos aéreos no tripulados 


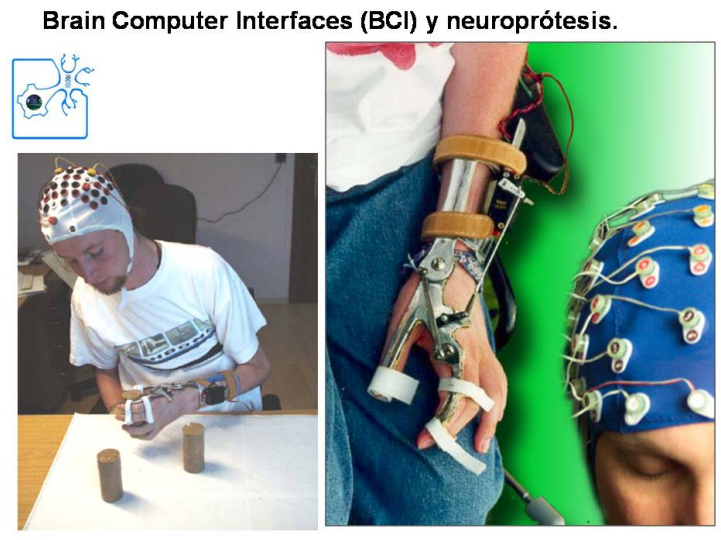

Figura 5.7: Un sujeto hace uso del sistema BCI para controlar una prótesis para rehabilitación

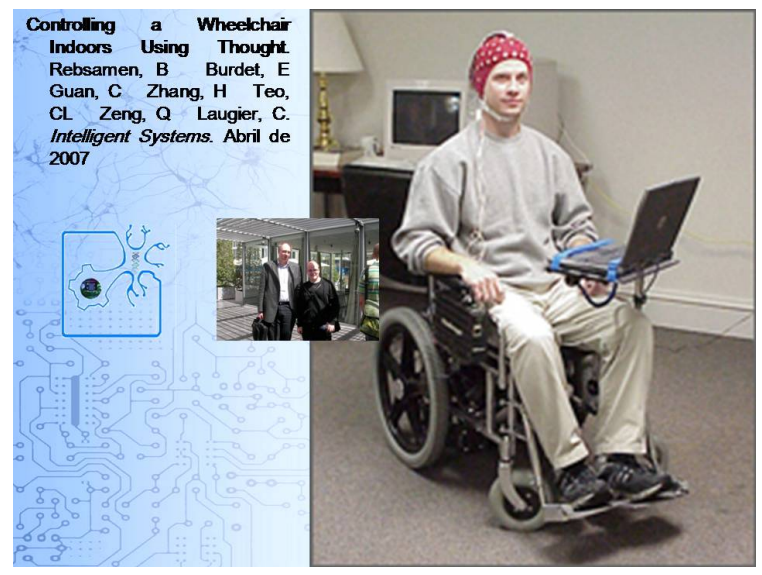

Figura 5.8: Sistema BCI para controlar el desplazamiento de una silla de ruedas

del usuario toma el control de un sujeto como si de su propio cuerpo se tratase. La Unión Europea ha financiado en los últimos meses proyectos relacionados con la telepresencia y el control de avatares. Es el caso del trabajo que se está desarrollando por parte del Laboratorio de Virtualidad Avanzada (AVL) de Israel dirigido por Doron Friedman. Los investigadores del AVL sostienen que es posible controlar un ávatar activando las áreas cerebrales correctas en cada momento. Los componentes del AVL han profundizado incluso en aspectos emocionales, llegando a preguntarse por qué las personas siguen teniendo preferencia por encuentros reales, a pesar del avance de la telefonía móvil y las videoconferencias. En este sentido, afirman que 


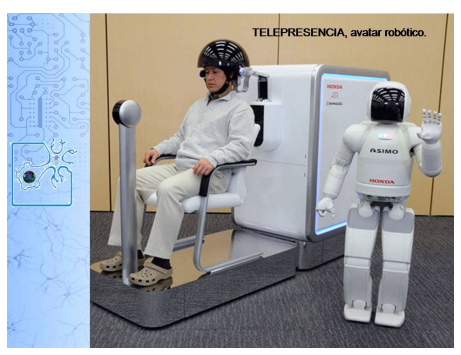

(a)

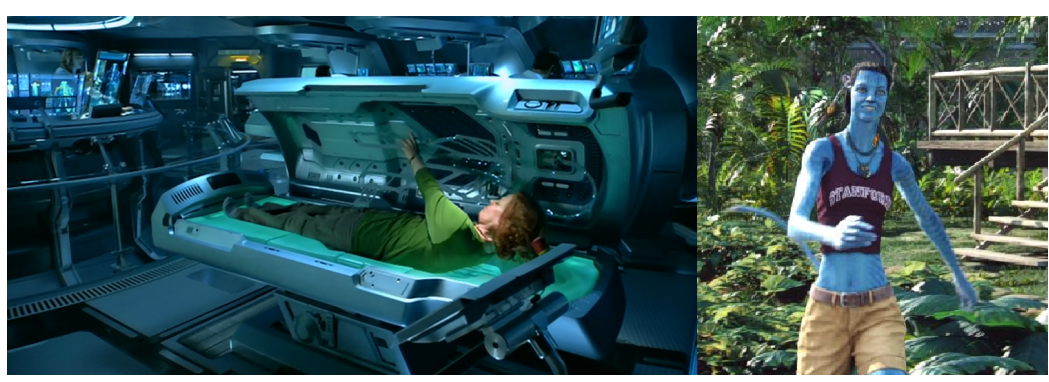

(b)

Figura 5.9: (a) Sistema BCI para el control completo de las funciones de un robot antropomorfo (b) Extracto de la película 'Ávatar'. El sujeto es capaz de controlar un ávatar a través de una interfaz cerebro-máquina.

algo debe perderse en esa comunicación virtual para que esto ocurra y trabajan actualmente en una línea que permitiría desarrollar tecnología que hiciera sentir a los interlocutores como si de una reunión en persona se tratase.

Todos estos ejemplos demuestran el potencial que los sistemas BCI pueden desplegar cuando se combinan con otros dispositivos complementarios. Lo expuesto anteriormente evidencia el esfuerzo de la comunidad científica para desarrollar sistemas BCI que puedan ser utilizados fuera de los laboratorios de investigación. Este es uno de los retos más importantes a los que se enfrenta esta tecnología. La mejora de los algoritmos de clasificación, el perfeccionamiento de los electrodos de adquisición de señal y la formulación de nuevos modelos de funcionamiento permitirán desarrollar una nueva generación de dispositivos BCI con altas precisiones, para su utilización fuera de los laboratorios.

\subsubsection{GoogleGlass como actuador en sistemas BCI}

La comunidad médica se esta preparando para un futuro en el que la información sobre la salud estará disponible de un vistazo. El sistema GoogleGlass (Figura 5.10) parece un par de gafas sin lentes y posee una pequeña computadora junto con una camara montada en el interior del marco. Colocado en la esquina superior derecha, el usuario dispone de una pantalla holográfica que parece flotar frente a él. El usuario interactúa con las GoogleGlass mediante comandos de voz, movimientos de la cabeza e incluso una barra tactil en uno de los laterales. Este dispositivo puede to- 


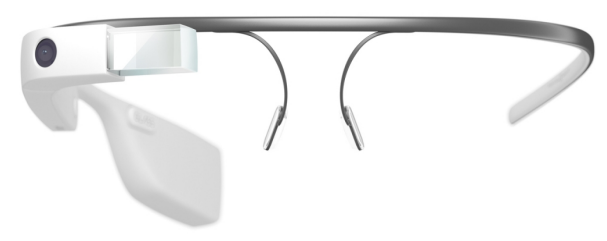

Figura 5.10: Dispositivo GoogleGlass utilizado en el presente desarrollo

mar imágenes, realizar video llamadas y permite al usuario realizar búsquedas web sin utilizar las manos. Las GoogleGlass están siendo probadas para su posible uso en aplicaciones médicas como formación a distancia, retransmisión de cirujías para estudiantes de medicina o revisión de datos de laboratorio sin apartar la vista del paciente. Algunos profesionales de la medicina han incorporado este dispositivo como apoyo para la visualización de datos obtenidos desde dispositivos médicos, otros han utilizado este sistema para permitir a los estudiantes seguir las intervenciones quirúrjicas desde la perspectiva del cirujano [35].

Estudios recientes [303] afirman que el futuro ha llegado de la mano de este tipo de dispositivos, entre los que se encuentran las GoogleGlass. Segun los autores, en poco tiempo, será posible encontrar personas en calles, centros educativos, fiestas, etc. utilizando este tipo de dispositivos. El análisis en este caso va mas allá de las consideraciones anteriores en las que este dispositivo era considerado únicamente como una cómoda cámara con conexión a internet o un display que mostraba información de forma confortable. En el citado articulo se diserta sobre la evolución y caracteristicas de las GoogleGlass asi como de otros dispositivos inteligentes que el usuario puede llevar puestos.

El 22 de Mayo de 2014, una cirugía realizada en el Royal London Hospital se convirtió en la primera cirugía retransmitida en directo a través de las GoogleGlass. El encargado de realizar dicha operación, Shafi Ahmed, declaró posteriormente que gracias a las GoogleGlass es posible realizar un gran servicio a los futuros cirujanos. El médico, afirmaba que gracias a este dispositivo es posible introducir a los futuros médicos en el interior del teatro de operaciones, permitiendo suministrar una forma- 
ción de gran calidad que repercutirá en un futuro con una mejor calidad asistencial. Las GoogleGlass fueron utilizadas por el médico durante toda la operación, consistente en extirpar tejido canceroso de ciertas partes del paciente. Esta cirujía fue seguida por 13.000 estudiantes de medicina de 115 países a través de computadoras, tabletas y teléfonos inteligentes. El sistema permitía que los espectadores pùdieran plantear cuestiones directamente al cirujano que iban apareciendo en la esquina inferior izquierda del display de las GoogleGlass del cirujano, sin entorpecer el campo visual del médico. El cirujano podía responder a las cuestiones planteadas sobre la marcha de forma verbal, siendo escuchadas por todos los seguidores en tiempo real. Más del $90 \%$ de los participantes en este experimento se mostraron favorables a que este tipo de aprendizaje forme parte del proceso de enseñanza. Según el presidente del Royal London Hospital, este sistema tiene un gran potencial para enseñar a cirujanos de todo el mundo nuevas técnicas quirúrjicas, realizadas por cada uno de los expertos en cada campo [304]. Las GoogleGlass han sido utilizadas también durante otras cirujías relacionadas con el aparato digestivo [305]

Los ejemplos anteriores muestran casos concretos en los que se ha conseguido vislumbrar el potencial evidente que poseen las GoogleGlass utilizadas en distintos entornos. Este dispositivo ha conseguido revolucionar los ámbitos en los que ha irrumpido, introduciendo nuevas prestaciones que hasta ahora eran difíciles de imaginar. La integración de las GoogleGlass en un nuevo modelo BCI, generará una revolución en este campo con mayor alcance que las descritas anteriormente.

\subsubsection{EMOTIV EPOC y GoogleGlass}

$\mathrm{Al}$ contrario de lo que se podría pensar, los dispositivos BCI portátiles no sólo han sido utilizados como elementos demostrativos, sino que algunos investigadores también los han utilizado para llevar a cabo investigaciones clínicas de forma exitosa. Recientemente [306] se desarrolló un sistema en el que un paciente con amputación de uno de sus miembros puede aprender a manipular a intención una prótesis virtual mediante un sistema BCI. A través de un sistema de realidad aumentada, se consigue transmitir a los sentidos del sujeto la sensación de que el modelo simulado es parte de su propio cuerpo, de esta forma se consigue generar un movimiento voluntario y natural. Para captar las señales electroencefalográficas se utilizó un dispositivo BCI 
comercial, el Emotiv EPOC, un neuroheadset inalámbrico de alta resolución para la adquisición de señales EEG. Analizando los resultados, puede concluirse que el sistema de rehabilitación propuesto es relativamente fácil de utilizar por personas sanas, con tiempos de entrenamiento que rondan los 30 minutos. El desarrollo del motor de clasificación permitió tener un mayor control sobre los parámetros del procesamiento y detección de patrones en las señales, donde se obtuvo hasta un $82.1 \%$ de precisión en la clasificación. Estas señales neuronales detectadas y procesadas, fueron utilizadas para descifrar la intención de cerrar o abrir un modelo virtual de una mano que se mostraba al sujeto adherida a su muñón mediante técnicas de realidad aumentada. Este entorno brindaba una retroalimentación visual al sujeto que contribuía a reducir neurologicamente el dolor del miembro fantasma.

El nivel de estres de un sujeto también ha sido objeto de medida a través de un dispositivo BCI portatil [307]. Utilizando un dispositivo NeuroSky Mindset se ha monitorizado la respuesta emocional de un sujeto durante la ejecución de tareas que inducían estres psicológico. Mediante este experimento se ha investigado la posibilidad de medir y categorizar el nivel de estres de los sujetos utilizando dispositivos de bioseñales sometiendo al sujeto a dos tipos de tests psicológicos. Por un lado se sometió a 20 sujetos a un test en el que existe un conflicto entre la palabra escrita y el color en el que esta escrita, conocido como test stroop. Por otro lado, a 17 sujetos se les sometió al test de las torres de hanoi. Todo ello con el objetivo de examinar la atención directa y sostenida durante la ejecución de estos tests y su correlación con el nivel de estrés generado durante la tarea. Los resultados mostraron que para la mayor parte de sujetos, cuanto menos atención mostraba, menor era su nivel de estress. En este sentido, el nivel de estres era mayor cuanto mayor nivel de atención mostraba el sujeto. A medida que el nivel de dificultad de la tarea se incrementaba, algunos sujetos comenzaban a perder interés y reducir su nivel de atención. Como concsecuencia de esto, decrecia el nivel de estres.

Otro de los campos analizados para el desarrollo de sistemas BCI ha sido la industria. En este sentido se ha estudiado la posibilidad de introducir sistemas BCI para ser utilizado en entornos industriales. Algunos autores [308] han resumido los progresos recientes desde la perspectiva de la robótica industrial, evaluando los nuevos productos comerciales en el campo del BCI. En este estudio se abordan nuevas 
vias para el uso de sistemas BCI presentando potenciales aplicaciones interesantes para sistemas robóticos industriales.

Se dispone de una cantidad limitada de trabajos relacionados con la conexión entre los dispositivos de monitorización cerebral y otros dispositivos como teléfonos inteligentes. A pesar de su gran calidad de señal, son todavia muy caros y poco versátiles a la hora de utilizarlos fuera del entorno de los laboratorios de investigación. Sin embargo, otros dispositivos portatiles como EPOC Emotiv o NeuroSky son más faciles de utilizar, más baratos y estan mas orientados a este tipo de aplicaciones a pesar de introducir un mayor ruido. Algunos autores [302] afirman que la conexión entre un dispositivo EEG como Emotiv EPOC y un actuador como un teléfono inteligente no puede despreciarse considerándolo un simple ejercicio de ingenieria. Precisamente por eso, se considera que este tipo de sistemas constituyen desarrollos importantes debido a su simplicidad y bajo coste. Ademas, abren nuevas oportunidades y retos en la sensorización cerebral y la seguridad de dichos datos. Monitorizar de forma inalámbrica los datos cerebrales de un sujeto puede tener ciertos riesgos si no se hace con las suficientes garantías de seguridad. Hacerlo en una red no segura, podría dar lugar a que dicha información fuese monitorizada por un tercero y con la tecnologia adecuada puede reconstruir a partir de la señal cerebral, las intenciones del usuario. Al margen de consideraciones legales, se abren nuevas posibilidades para el desarrollo de sistemas BCI más robustos, versátiles y faciles de utilizar fuera del laboratorio.

Los ejemplos anteriores demuestran la viabilidad de utilizar dispositivos portátiles de monitorización como parte de sistemas BCI con un cierto nivel de robustez. Las prestaciones que ofrecen los dispositivos de monitorización de señales EEG portátiles pueden ser evaluados y comparados en función de diversos parámetros como su capacidad, portabilidad, resolución, escalabilidad, fiabilidad, muestreo, o compatibilidad. Comercialmente existen varios tipos de hardware con características diferentes, que se ajustan a distintas necesidades y aplicaciones. Entre los más representativos del mercado se destacan cuatro de ellos debido a su calidad, precio y popularidad en el campo de la investigación académica: g.BCIsys con g.USBamp de g.tec (G.tec, 2011) producidos en Austria, EEG Powerlab Data Adquisition System (ADInstruments, 2012) producido en Nueva Zelanda, Emotiv EPOC EEG (Emotiv, 


\begin{tabular}{ll}
\hline \hline Criterios & Detalle \\
\hline \hline Capacidad & Cantidad de canales de adquisición \\
Asequibilidad & Precio, distribución local, Promoción \\
Portabilidad & Usabilidad, alámbrico/inalámbrico, tipos de electrodos \\
Resolución & Espacial y el número de bits de la señal de lectura \\
Muestreo & Frecuencia de adquisición, amplificación y filtrado \\
Compatibilidad & Facilidad de integración con distintas plataformas de software \\
Fiabilidad & Marca, garantía, trabajos realizados, referencias \\
Flexibilidad & Tipo de sensores y señales que puede adquirir \\
Escalabilidad & Librerias y funcionalidades que permitan procesar en diferentes eta- \\
& pas las señales, ya sean desde su obtención como señales puras hasta \\
& ya clasificadas
\end{tabular}

Cuadro 5.1: Definición de los distintos criterios de evaluación a los que se han sometido los dispositivos evaluados [306]

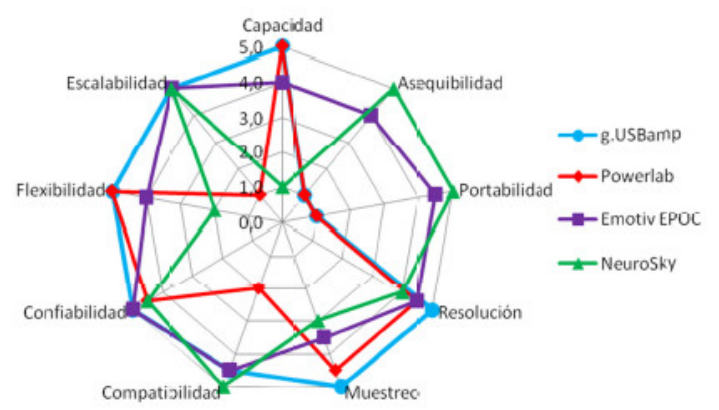

Figura 5.11: Comparativa de los resultados obtenidos por cada uno de los sistemas evaluados [306]

2012) producido en Australia y Neurosky Mindwave (Neurosky, 2012) producido en Estados Unidos. El Cuadro 5.2 muestra una comparativa de los distintos dispositivos de monitorización disponibles. Se fijan unos criterios de evaluación comunes para todos los sistemas analizando su capacidad, asequibilidad en el mercado, resolución, portabilidad, muestreo, compatibilidad, fiabilidad, flexibilidad y escalabilidad.

Las cuatro alternativas se clasificaron con base a estos parámetros, evaluando cada uno de los parámetros de 0 a 5 puntos como queda reflejado tanto en la Figura 5.11 como en el Cuadro 5.2. Tras la evaluación, el Emotiv EPOC (Adelson, 2011) ha sido el dispositivo que mejores resultados ha obtenido y por ello ha sido 


\begin{tabular}{llllll}
\hline \hline Criterios & Pesos & g.USBamp & PowerLab & $\begin{array}{l}\text { EPOC } \\
\text { Emotiv }\end{array}$ & Neurosky \\
\hline \hline Capacidad & $10 \%$ & 5,0 & 5,0 & 4,0 & 1,0 \\
Asequibilidad & $20 \%$ & 1,0 & 1,0 & 4,0 & 5,0 \\
Portabilidad & $18 \%$ & 1,0 & 1,0 & 4,5 & 5,0 \\
Resolución & $5 \%$ & 5,0 & 4,5 & 4,5 & 5,0 \\
Muestreo & $5 \%$ & 5,0 & 4,5 & 3,5 & 3,0 \\
Compatibilidad & $15 \%$ & 4,5 & 2,0 & 4,5 & 5,0 \\
Fiabilidad & $10 \%$ & 5,0 & 4,5 & 5,0 & 4,5 \\
Flexibilidad & $5 \%$ & 5,0 & 5,0 & 4,0 & 2,0 \\
Escalabilidad & $12 \%$ & 5,0 & 1,0 & 5,0 & 5,0 \\
\hline \hline Total Ponderado & $100 \%$ & 3.41 & 2.45 & 4.39 & 4.25 \\
\hline
\end{tabular}

Cuadro 5.2: Resultado de la evaluación realizada a cada uno de los dispositivos en función de cada uno de los parámetros del Cuadro 5.2 [306]

clasificado como el dispositivo que mayores prestaciones ofrece.

La evaluación anterior sitúa en una clara posición de ventaja al dispositivo Emotiv EPOC. Este monitorizador BCI es un neuroheadset inalámbrico de alta resolución para la adquisición y procesamiento de señales EEG. El registro se realiza a través de 14 canales correspondientes a 14 electrodos secos colocados en la parte superior de la cabeza de acuerdo al sistema 10-20 de posicionamiento de electrodos para EEG, ilustrado en la Figura 2.

El dispositivo Emotiv EPOC ha sido utilizado en el pasado por diversos autores de forma satisfactoria [309] [310] [311]. Algunos autores [309], utilizaron este dispositivo como base para diseñar un sistema BCI cuyo objetivo era el controlar un robot humanoide utilizando señales obtenidas a partir de los 14 electrodos de Emotiv EPOC. Los sujetos seleccionan la dirección fijando la mirada en un grupo de LEDs que representa la dirección deseada. Para detectar la componente frecuencial dominante se utilizó transformada rápida de fourier (FFT) y modelos gausianos. A partir del resultado obtenido, el robot recibe una señal de control con la dirección seleccionada por el usuario a través del sistema BCI. Los resultados experimentales confirman que el sistema funciona correctamente. La precisión media en la decodificación de la dirección fue del $75 \%$, sin necesidad de realizar ningún tipo de entrenamiento previo.

Algunos autores han comparado el funcionamiento de Emotiv EPOC con otros 
sistemas EEG más sofisticados [312], utilizados normalmente en los laboratorios médicos. En el contexto de un sistema BCI P300 se han evaluado las prestaciones de Emotiv EPOC frente a las del sistema médico ANT (Advanced Neuro Technology, ANT, Enschede, The Netherlands). Este sistema EEG, está compuesto por 128 electrodos $\mathrm{Ag} / \mathrm{AgCl}$, protegidos del ruido exterior y con el correspondiente amplificador DC que puede alcanzar tasas de muestreo de $2048 \mathrm{~Hz}$ mientras se mantiene la impedancia por debajo de $20 \mathrm{k} \Omega$ gracias al uso de gel para electrodos. Tras comparar ambos sistemas en un mismo contexto, los resultados en terminos de precisión muestran que Emotiv EPOC puede sustituir a otros sistemas de mayor capacidad en determinadas aplicaciones en las que un fallo no genera consecuencias graves. Algunos de los resultados arrojados por este estudio son concluyentes. Globalmente, los resultados obtenidos por Emotiv EPOC parecen ser ligeramente peores que los obtenidos por el sistema ANT. En algunos casos, como en condiciones en las que el sujeto esta sentado o caminando durante la comparación basada en Validación cruzada en k segmentos (k-fold cross validation explicada en el Capítulo 3), la diferencia puede deberse a una mayor relación señal a ruido del sistema médico. En el caso de los conjuntos de test, la diferencia es debida probablemente a aspectos relacionados con el contexto P300.

\subsection{Modelo combinado BCI - GoogleGlass}

En el marco de la presente tesis doctoral se ha desarrollado un sistema complementario con el descrito en el Capítulo 4, que abre una nueva línea para mejorar los sistemas BCI utilizados fuera del laboratorio. A través de un desarrollo software específico, ha sido posible comunicar directamente un dispositivo BCI portátil con las GoogleGlass. Esta comunicación permite el control directo de algunas acciones de las GoogleGlass a través de las señales EEG del sujeto. Un ejemplo sobre la utilización real de este sistema puede observarse en la Figura 5.12. La comunicación establecida entre las GoogleGlass y el sistema Emotiv EPOC, permite al usuario controlar la cámara de video y hacer fotografías cuando el usuario cambia sus estados mentales. El usuario debe concentrarse para que las GoogleGlass realicen una fotografía en dicho instante. El sistema también es capaz de detectar otros estados mentales y 


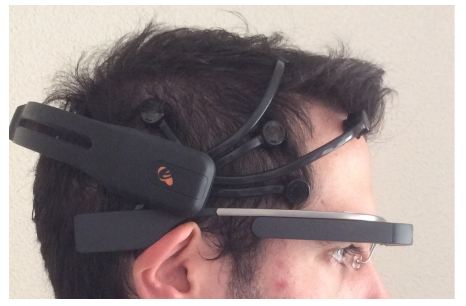

(a)

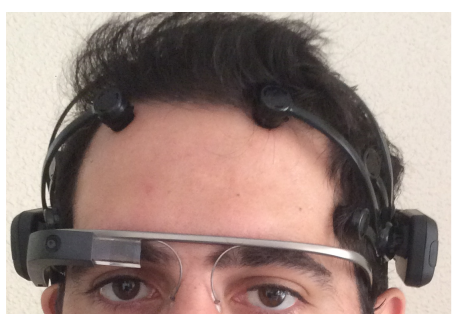

(b)

Figura 5.12: Un sujeto hace uso del desarrollo software que permite controlar algunas funciones de las GoogleGlass mediante señales cerebrales

grabar vídeo a voluntad del usuario de distintas duraciones. La comunicación que se establece entre las GoogleGlass y el sistema BCI no sólo permite controlar las GoogleGlass mediante señales EEG, sino que también es posible utilizar este canal para otras funciones complementarias.

Combinando este desarrollo junto con el anteriormente mencionado [288], se abre una línea de desarrollo futuro con la que sacar los sistemas BCI de los laboratorios. El sistema propuesto está basado en incorporar al modelo de la Figura 5.1 un sistema BCI portátil, que esté apoyado por sistemas de realidad aumentada a traves de GoogleGlass. Para conseguir la utilización de este sistema como prótesis robótica que permita restituir parcialmente el movimiento de una persona impedida, es necesario aumentar las funcionalidades y mejorar la capacidad de selección del objetivo deseado por el usuario de entre varias posibilidades. La combinación entre el sistema [288] y el modelo desarrollado en la presente tesis, abre una línea futura para el desarrollo de nuevas protesis robóticas inteligentes. El perfeccionamiento de este modelo, abre la puertra a una nueva generación de neuroprótesis basadas en BCI, realidad aumentada, visión artificial y robótica inteligente.

En la presente tesis doctoral se plantea un nuevo modelo de BCI que incorpore las posibidades que ofrecen las GoogleGlass para interaccionar con el entorno. Las neuroprótesis actuales poseen ciertas limitaciones para interactuar con el entorno con cierta precisión. Desarrollar un modelo BCI completo integrando a las GoogleGlass, permitiría superar muchas de las limitaciones que impiden su utilización fuera de los laboratorios. Los resultados obtenidos en la presente tesis doctoral no hacen sino confirmar el margen de mejora que todavía existe para mejorar la precisión de los 


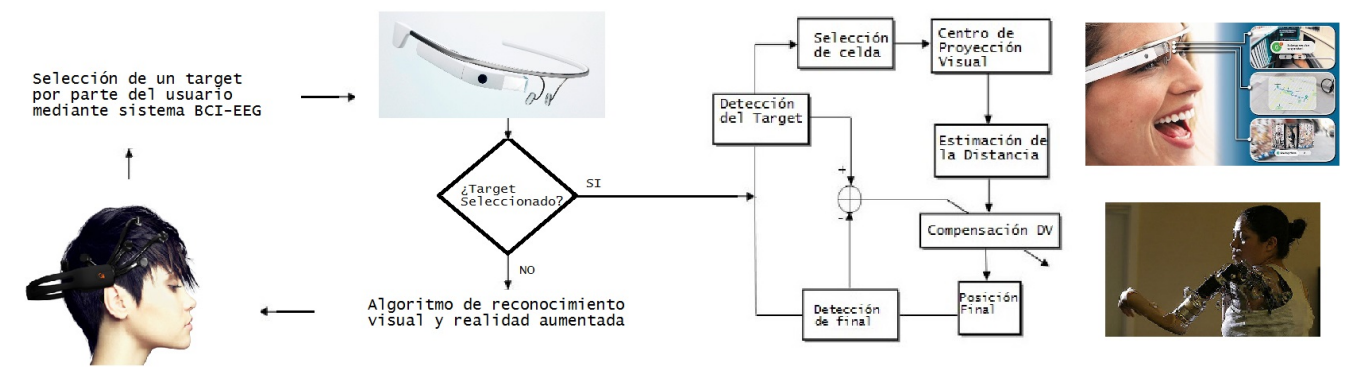

Figura 5.13: Modelo de neuroprótesis propuesto utilizando GoogleGlass, realidad aumentada y el sistema EMOTIV Epoc

sistemas BCI no invasivos a partir de señales EEG obtenidas en entornos tridimensionales. La comunicación establecida en la presente tesis doctoral entre las GoogleGlass y un sistemas BCI portátil, permitirá desarrollar modelos como el descrito en la Figura 5.13. Para aumentar la robustez del sistema, se propone la incorporación de un sistema de realidad aumentada basado en desarrollos probados y publicados en la literatura científica [306]. Incorporar realidad aumentada al sistema permitirá que las GoogleGlass reconozcan mejor los distintos elementos que forman parte del entorno del sujeto. El reconocimiento de los targets y la identificación de los elementos robóticos del sistema se facilita mediante la utilización de la realidad aumentada. El display de las GoogleGlass mostrará al usuario distintos targets para que este pueda seleccionar uno de ellos mediante la actividad EEG. Una vez seleccionado el target, el control del movimiento hasta el Target se realiza siguiendo el flujograma de la Figura 5.13.

En este trabajo se han abierto líneas importantes para el desarrollo futuro de los sistemas BCI. La alta precisión obtenida en entornos tridimensionales abre la posibilidad a seguir mejorando estos sistemas BCI no invasivos para obtener valores mayores de información decodificada. El modelo de neuroprótesis propuesto utilizando GoogleGlass, realidad aumentada y el sistema EMOTIV Epoc (Figura 5.13 abre una nueva línea de actuación para mejorar las prestaciones y la usabilidad de los sistemas BCI. El desarrollo futuro de los sistemas BCI debe pasar por el aumento de la información decodificada (DI) y por la integración de nuevos dispositivos al modelo BCI, que permitan aumentar la independencia entre el laboratorio y el sistema 
robótico. 


\section{Bibliografía}

[1] G Schalk, J Kubanek, KJ Miller, NR Anderson, EC Leuthardt, JG Ojemann, D Limbrick, D Moran, LA Gerhardt, and JR Wolpaw. Decoding twodimensional movement trajectories using electrocorticographic signals in humans. Journal of neural engineering, 4(3):264, 2007.

[2] Gerwin Schalk and Eric C Leuthardt. Brain-computer interfaces using electrocorticographic signals. Biomedical Engineering, IEEE Reviews in, 4:140-154, 2011.

[3] Wolfgang Klimesch. Eeg alpha and theta oscillations reflect cognitive and memory performance: a review and analysis. Brain research reviews, 29(2):169195, 1999.

[4] Kyungmin Su and Kay A. Robbins. A framework for content-based retrieval of eeg with applications to neuroscience and beyond. Proceedings of ... International Joint Conference on Neural Networks / co-sponsored by Japanese Neural Network Society (JNNS) ... [et al.]. International Joint Conference on Neural Networks, pages 1-8, 2013.

[5] Guillermo Gallegos-Ayala, Adrian Furdea, Kouji Takano, Carolin A. Ruf, Herta Flor, and Niels Birbaumer. Brain communication in a completely locked-in patient using bedside near-infrared spectroscopy. Neurology, Apr 2014.

[6] Grupo Orgánico de Enfermedades No Transmisibles and Salud Mental. Envejecimiento activo: un marco político. Rev Esp Geriatr Gerontol, 37(S2):74-105, 2002. 
[7] Bram van de Laar, Boris Reuderink, Danny Plass-Oude Bos, and Dirk Heylen. Evaluating user experience of actual and imagined movement in bci gaming. 2010 .

[8] Cuntai Guan, Manoj Thulasidas, and Jiankang Wu. High performance p300 speller for brain-computer interface. In Biomedical Circuits and Systems, 2004 IEEE International Workshop on, pages S3-5. IEEE, 2004.

[9] Christoph Guger, Shahab Daban, Eric Sellers, Clemens Holzner, Gunther Krausz, Roberta Carabalona, Furio Gramatica, and Guenter Edlinger. How many people are able to control a p300-based brain-computer interface (bci)? Neuroscience letters, 462(1):94-98, 2009.

[10] William J Ray and Harry W Cole. Eeg alpha activity reflects attentional demands, and beta activity reflects emotional and cognitive processes. Science, 228(4700):750-752, 1985.

[11] Kannathal Natarajan, Rajendra Acharya, Fadhilah Alias, Thelma Tiboleng, and Sadasivan K Puthusserypady. Nonlinear analysis of eeg signals at different mental states. BioMedical Engineering OnLine, 3(1):7, 2004.

[12] Thalía Harmony, Thalía Fernández, Juan Silva, Jorge Bernal, Lourdes DíazComas, Alfonso Reyes, Erzsébet Marosi, Mario Rodríguez, and Miguel Rodríguez. Eeg delta activity: an indicator of attention to internal processing during performance of mental tasks. International journal of psychophysiology, 24(1):161-171, 1996.

[13] Jun Lv and Yuanqing Li. Decoding hand movement velocities from eeg signals during a continuous drawing task. In Fuzzy Systems and Knowledge Discovery (FSKD), 2010 Seventh International Conference on, volume 5, pages 21862189. IEEE, 2010.

[14] G Schalk, J Kubanek, KJ Miller, NR Anderson, EC Leuthardt, JG Ojemann, D Limbrick, D Moran, LA Gerhardt, and JR Wolpaw. Decoding twodimensional movement trajectories using electrocorticographic signals in humans. Journal of neural engineering, 4(3):264, 2007. 
[15] Ethan Buch, Cornelia Weber, Leonardo G Cohen, Christoph Braun, Michael A Dimyan, Tyler Ard, Jurgen Mellinger, Andrea Caria, Surjo Soekadar, Alissa Fourkas, et al. Think to move: a neuromagnetic brain-computer interface (bci) system for chronic stroke. Stroke, 39(3):910-917, 2008.

[16] Jürgen Mellinger, Gerwin Schalk, Christoph Braun, Hubert Preissl, Wolfgang Rosenstiel, Niels Birbaumer, and Andrea Kübler. An meg-based braincomputer interface (bci). Neuroimage, 36(3):581-593, 2007.

[17] N Jeremy Hill, Thomas Navin Lal, M Schroder, Thilo Hinterberger, Barbara Wilhelm, Femke Nijboer, Ursula Mochty, Guido Widman, CE Elger, Bernhard Scholkopf, et al. Classifying eeg and ecog signals without subject training for fast bci implementation: comparison of nonparalyzed and completely paralyzed subjects. Neural Systems and Rehabilitation Engineering, IEEE Transactions on, 14(2):183-186, 2006.

[18] Adam G Rouse and Daniel W Moran. Neural adaptation of epidural electrocorticographic (eecog) signals during closed-loop brain computer interface (bci) tasks. In Engineering in Medicine and Biology Society, 2009. EMBC 2009. Annual International Conference of the IEEE, pages 5514-5517. IEEE, 2009.

[19] Martin Lotze, Pedro Montoya, Michael Erb, Ernst Hülsmann, Herta Flor, Uwe Klose, Niels Birbaumer, and Wolfgang Grodd. Activation of cortical and cerebellar motor areas during executed and imagined hand movements: an fmri study. Journal of cognitive neuroscience, 11(5):491-501, 1999.

[20] Ranganatha Sitaram, Haihong Zhang, Cuntai Guan, Manoj Thulasidas, Yoko Hoshi, Akihiro Ishikawa, Koji Shimizu, and Niels Birbaumer. Temporal classification of multichannel near-infrared spectroscopy signals of motor imagery for developing a brain-computer interface. NeuroImage, 34(4):1416-1427, 2007.

[21] Peter Brunner, Anthony L Ritaccio, Joseph F Emrich, Horst Bischof, and Gerwin Schalk. Rapid communication with a "p300" matrix speller using electrocorticographic signals (ecog). Frontiers in neuroscience, 5:5, 2011. 
[22] G Schalk, KJ Miller, NR Anderson, JA Wilson, MD Smyth, JG Ojemann, DW Moran, JR Wolpaw, and EC Leuthardt. Two-dimensional movement control using electrocorticographic signals in humans. Journal of neural engineering, 5(1):75, 2008.

[23] Pradeep Shenoy, Kai J Miller, Jeffrey G Ojemann, and Rajesh PN Rao. Generalized features for electrocorticographic bcis. Biomedical Engineering, IEEE Transactions on, 55(1):273-280, 2008.

[24] Tobias Pistohl, Tonio Ball, Andreas Schulze-Bonhage, Ad Aertsen, and Carsten Mehring. Prediction of arm movement trajectories from ecog-recordings in humans. Journal of neuroscience methods, 167(1):105-114, 2008.

[25] Laura Kauhanen, Tommi Nykopp, Janne Lehtonen, P Jylanki, Jukka Heikkonen, Pekka Rantanen, Hannu Alaranta, and Mikko Sams. Eeg and meg braincomputer interface for tetraplegic patients. Neural Systems and Rehabilitation Engineering, IEEE Transactions on, 14(2):190-193, 2006.

[26] Stephan Waldert, Hubert Preissl, Evariste Demandt, Christoph Braun, Niels Birbaumer, Ad Aertsen, and Carsten Mehring. Hand movement direction decoded from meg and eeg. The Journal of neuroscience, 28(4):1000-1008, 2008.

[27] Karim Jerbi, Samson Freyermuth, Lorella Minotti, Philippe Kahane, Alain Berthoz, and Jean-Philippe Lachaux. Watching brain tv and playing brain ball: Exploring novel bci strategies using real-time analysis of human intracranial data. International review of neurobiology, 86:159-168, 2009.

[28] Thorsten Oliver Zander. Utilizing brain-computer interfaces for humanmachine systems. PhD thesis, Universitätsbibliothek der Technischen Universität Berlin, 2011.

[29] Lin He, Youpan Hu, Yuanqing Li, and Daoli Li. Channel selection by rayleigh coefficient maximization based genetic algorithm for classifying single-trial motor imagery eeg. Neurocomputing, 121:423-433, 2013.

[30] Gerwin Schalk, Dennis J McFarland, Thilo Hinterberger, Niels Birbaumer, and Jonathan R Wolpaw. Bci2000: a general-purpose brain-computer interface 
(bci) system. Biomedical Engineering, IEEE Transactions on, 51(6):1034-1043, 2004.

[31] Gert Pfurtscheller, Christa Neuper, Gernot R Müller, Bernhard Obermaier, Gunter Krausz, Alois Schlögl, Reinhold Scherer, Bernhard Graimann, Claudia Keinrath, Dimitris Skliris, et al. Graz-bci: state of the art and clinical applications. IEEE transactions on neural systems and rehabilitation engineering: a publication of the IEEE Engineering in Medicine and Biology Society, 11(2):177-180, 2003.

[32] E Rocon, AF Ruiz, JL Pons, José M Belda-Lois, and JJ Sánchez-Lacuesta. Rehabilitation robotics: a wearable exo-skeleton for tremor assessment and suppression. pages 2271-2276, 2005.

[33] Shah Mihir Rajesh. Design of human exo-skeleton suit for rehabilitation of hemiplegic people. Procedia Engineering, 51:544-553, 2013.

[34] Atilla Kilicarslan, Saurabh Prasad, Robert G Grossman, and Jose L ContrerasVidal. High accuracy decoding of user intentions using eeg to control a lowerbody exoskeleton. In Engineering in Medicine and Biology Society (EMBC), 2013 35th Annual International Conference of the IEEE, pages 5606-5609. IEEE, 2013.

[35] Wendy Glauser. Doctors among early adopters of google glass. Canadian Medical Association Journal, pages cmaj-109, 2013.

[36] Jonathan R Wolpaw, Dennis J McFarland, Gregory W Neat, and Catherine A Forneris. An eeg-based brain-computer interface for cursor control. Electroencephalography and clinical neurophysiology, 78(3):252-259, 1991.

[37] Andrea Kübler, Femke Nijboer, Jürgen Mellinger, Theresa M Vaughan, Hannelore Pawelzik, Gerwin Schalk, DJ McFarland, Niels Birbaumer, and Jonathan R Wolpaw. Patients with als can use sensorimotor rhythms to operate a brain-computer interface. Neurology, 64(10):1775-1777, 2005. 
[38] Niels Birbaumer, Nimr Ghanayim, Thilo Hinterberger, Iver Iversen, Boris Kotchoubey, Andrea Kübler, Juri Perelmouter, Edward Taub, and Herta Flor. A spelling device for the paralysed. Nature, 398(6725):297-298, 1999.

[39] Eleanor A Curran and Maria J Stokes. Learning to control brain activity: a review of the production and control of eeg components for driving braincomputer interface (bci) systems. Brain and cognition, 51(3):326-336, 2003.

[40] Jonathan R Wolpaw. Brain-computer interfaces: progress, problems, and possibilities. In Proceedings of the 2nd ACM SIGHIT International Health Informatics Symposium, pages 3-4. ACM, 2012.

[41] SG Mason, MM Moore Jackson, and GE Birch. A general framework for characterizing studies of brain interface technology. Annals of Biomedical Engineering, 33(11):1653-1670, 2005.

[42] SG Mason, A Bashashati, M Fatourechi, KF Navarro, and GE Birch. A comprehensive survey of brain interface technology designs. Annals of biomedical engineering, 35(2):137-169, 2007.

[43] Christoph Guger, Alois Schlogl, Christa Neuper, Dirk Walterspacher, Thomas Strein, and Gert Pfurtscheller. Rapid prototyping of an eeg-based braincomputer interface (bci). Neural Systems and Rehabilitation Engineering, IEEE Transactions on, 9(1):49-58, 2001.

[44] Yuanqing Li, Jinyi Long, Tianyou Yu, Zhuliang Yu, Chuanchu Wang, Haihong Zhang, and Cuntai Guan. An eeg-based bci system for 2-d cursor control by combining mu/beta rhythm and p300 potential. Biomedical Engineering, IEEE Transactions on, 57(10):2495-2505, 2010.

[45] Gernot R Müller-Putz, Reinhold Scherer, Gert Pfurtscheller, and Rüdiger Rupp. Eeg-based neuroprosthesis control: a step towards clinical practice. Neuroscience letters, 382(1):169-174, 2005.

[46] Brendan Z Allison and Jaime A Pineda. Erps evoked by different matrix sizes: implications for a brain computer interface (bci) system. Neural Systems and Rehabilitation Engineering, IEEE Transactions on, 11(2):110-113, 2003. 
[47] George Townsend, Bernhard Graimann, and Gert Pfurtscheller. Continuous eeg classification during motor imagery-simulation of an asynchronous bci. Neural Systems and Rehabilitation Engineering, IEEE Transactions on, 12(2):258-265, 2004.

[48] Gert Pfurtscheller, C Guger, G Müller, G Krausz, and C Neuper. Brain oscillations control hand orthosis in a tetraplegic. Neuroscience letters, 292(3):211214,2000 .

[49] Germán Rodríguez Bermúdez, Pedro José García Laencina, José Luis Roca González, Joaquín Roca González, and Joaquín Roca Dorda. Técnicas de reconocimiento de patrones para la clasificación de señales eeg en sistemas bci. 2012.

[50] Leonard J Trejo, Roman Rosipal, and Bryan Matthews. Brain-computer interfaces for 1-d and 2-d cursor control: designs using volitional control of the eeg spectrum or steady-state visual evoked potentials. Neural Systems and Rehabilitation Engineering, IEEE Transactions on, 14(2):225-229, 2006.

[51] JC Woestenburg, MN Verbaten, and JL Slangen. The removal of the eyemovement artifact from the eeg by regression analysis in the frequency domain. Biological psychology, 16(1):127-147, 1983.

[52] Edmund Wascher, Sven Hoffmann, Jessica Sänger, and Marc Grosjean. Visuospatial processing and the n1 component of the erp. Psychophysiology, 46(6):1270-1277, 2009.

[53] David Edward Goldberg et al. Genetic algorithms in search, optimization, and machine learning, volume 412. Addison-wesley Reading Menlo Park, 1989.

[54] Anusha Venkatakrishnan, Gerard E Francisco, and Jose L Contreras-Vidal. Applications of brain-machine interface systems in stroke recovery and rehabilitation. Current Physical Medicine and Rehabilitation Reports, 2(2):93-105, 2014 .

[55] Roman Krepki, Benjamin Blankertz, Gabriel Curio, and Klaus-Robert Müller. The berlin brain-computer interface (bbci)-towards a new communication 
channel for online control in gaming applications. Multimedia Tools and Applications, 33(1):73-90, 2007.

[56] Ahmed A Karim, Thilo Hinterberger, Jürgen Richter, Jürgen Mellinger, Nicola Neumann, Herta Flor, Andrea Kübler, and Niels Birbaumer. Neural internet: Web surfing with brain potentials for the completely paralyzed. Neurorehabilitation and Neural Repair, 20(4):508-515, 2006.

[57] Tihomir Surdilovic and Yan-Qing Zhang. Convenient intelligent cursor control web systems for internet users with severe motor-impairments. International journal of medical informatics, 75(1):86-100, 2006.

[58] Babak A Taheri, Robert T Knight, and Rosemary L Smith. A dry electrode for eeg recording. Electroencephalography and clinical neurophysiology, 90(5):376383, 1994.

[59] Florin Popescu, Siamac Fazli, Yakob Badower, Benjamin Blankertz, and KlausR Müller. Single trial classification of motor imagination using 6 dry eeg electrodes. PloS one, 2(7):e637, 2007.

[60] Giuseppe Moruzzi. The electrophysiological work of carlo matteucci. Brain research bulletin, 40(2):69-91, 1996.

[61] Francisco Villarejo Ortega. Tratamiento de la epilepsia. Ediciones Díaz de Santos, 1998.

[62] M Tudor, L Tudor, and KI Tudor. [hans berger (1873-1941)-the history of electroencephalography]. Acta medica Croatica: casopis Hravatske akademije medicinskih znanosti, 59(4):307-313, 2004.

[63] Hans Berger. Über das elektrenkephalogramm des menschen. European Archives of Psychiatry and Clinical Neuroscience, 87(1):527-570, 1929.

[64] LF Haas. Hans berger (1873-1941), richard caton (1842-1926), and electroencephalography. Journal of Neurology, Neurosurgery Eamp; Psychiatry, 74(1):9-9, 2003. 
[65] AE Kornmüller and R Janzen. Über die normalen bioelektrischen erscheinungen des menschlichen gehirns. European Archives of Psychiatry and Clinical Neuroscience, 110(2):224-252, 1939.

[66] W Grey Walter and VJ Dovey. Electro-encephalography in cases of sub-cortical tumour. Journal of neurology, neurosurgery, and psychiatry, 7(3-4):57, 1944.

[67] F Golla, S Graham, and W Grey Walter. The electro-encephalogram in epilepsy. The British Journal of Psychiatry, 83(343):137-155, 1937.

[68] Yunyong Punsawad, Yodchanan Wongsawat, and Manukid Parnichkun. Hybrid eeg-eog brain-computer interface system for practical machine control. In Engineering in Medicine and Biology Society (EMBC), 2010 Annual International Conference of the IEEE, pages 1360-1363. IEEE, 2010.

[69] Anton Nijholt. Bci for games: A "state of the art"survey. In Entertainment Computing-ICEC 2008, pages 225-228. Springer, 2009.

[70] Wenjie Xu, Cuntai Guan, Chng Eng Siong, S Ranganatha, M Thulasidas, and Jiankang Wu. High accuracy classification of eeg signal. In Pattern Recognition, 2004. ICPR 2004. Proceedings of the 17th International Conference on, volume 2, pages 391-394. IEEE, 2004.

[71] Eiji Hoshi, Léon Tremblay, Jean Féger, Peter L Carras, and Peter L Strick. The cerebellum communicates with the basal ganglia. Nature neuroscience, 8(11):1491-1493, 2005.

[72] Frank A Middleton and Peter L Strick. Anatomical evidence for cerebellar and basal ganglia involvement in higher cognitive function. Science, 266(5184):458461, 1994.

[73] F Lopes da Silva and Ab Van Rotterdam. Biophysical aspects of eeg and magnetoencephalogram generation. Electroencephalography: Basic principles, clinical applications, and related fields, pages 107-125, 2005.

[74] Robin I Goldman, John M Stern, Jerome Engel Jr, and Mark S Cohen. Simultaneous eeg and fmri of the alpha rhythm. Neuroreport, 13(18):2487, 2002. 
[75] H Laufs, A Kleinschmidt, A Beyerle, E Eger, A Salek-Haddadi, C Preibisch, and K Krakow. Eeg-correlated fmri of human alpha activity. Neuroimage, 19(4):1463-1476, 2003.

[76] AS Gevins and RE Schaffer. A critical review of electroencephalographic (eeg) correlates of higher cortical functions. Critical reviews in bioengineering, 4(2):113-164, 1979.

[77] Paul Sauseng, Birgit Griesmayr, Roman Freunberger, and Wolfgang Klimesch. Control mechanisms in working memory: a possible function of eeg theta oscillations. Neuroscience \&3 Biobehavioral Reviews, 34(7):1015-1022, 2010.

[78] Gerard J Tortora, Bryan Derrickson, and Mario Dvorkin. Principios de anatomía y fisiología. 2006.

[79] F Valverde. Estructura de la corteza cerebral. organización intrínseca y análisis comparativo del neocortex. Rev Neurol, 34(8):758-780, 2002.

[80] Mahlon R DeLong. Primate models of movement disorders of basal ganglia origin. Trends in neurosciences, 13(7):281-285, 1990.

[81] Kim E Barrett and William F Ganong. Ganong fisiología médica. McGrau Hill Interamericana, 2010.

[82] Margaret A Kennard. Effects on eeg of chronic lesions of basal ganglia, thalamus and hypothalamus of monkeys. J. Neurophysiol, 6(5-6):405, 1943.

[83] Peter Brown. Oscillatory nature of human basal ganglia activity: relationship to the pathophysiology of parkinson's disease. Movement Disorders, 18(4):357363, 2003.

[84] Ivan Rektor, Robert Kuba, and Milan Brázdil. Interictal and ictal eeg activity in the basal ganglia: an seeg study in patients with temporal lobe epilepsy. Epilepsia, 43(3):253-262, 2002.

[85] DH Hubel and TN Wiesel. Anatomical demonstration of columns in the monkey striate cortex. Nature, 221(5182):747-750, 1969. 
[86] Sandrine Lefort, Christian Tomm, J-C Floyd Sarria, and Carl CH Petersen. The excitatory neuronal network of the $\mathrm{c} 2$ barrel column in mouse primary somatosensory cortex. Neuron, 61(2):301-316, 2009.

[87] Wilder Penfield and Edwin Boldrey. Somatic motor and sensory representation in the cerebral cortex of man as studied by electrical stimulation. Brain: A journal of neurology, 1937.

[88] Geoffrey D Schott. Penfield's homunculus: a note on cerebral cartography. Journal of neurology, neurosurgery, and psychiatry, 56(4):329, 1993.

[89] G Cheron, M Duvinage, C De Saedeleer, T Castermans, A Bengoetxea, M Petieau, K Seetharaman, T Hoellinger, B Dan, T Dutoit, et al. From spinal central pattern generators to cortical network: integrated bci for walking rehabilitation. Neural plasticity, 2012, 2012.

[90] Zheng Yang Chin, Kai Keng Ang, Chuanchu Wang, Cuntai Guan, and Haihong Zhang. Multi-class filter bank common spatial pattern for four-class motor imagery bci. In Engineering in Medicine and Biology Society, 2009. EMBC 2009. Annual International Conference of the IEEE, pages 571-574. IEEE, 2009.

[91] K Brodmann. Vergleichende Lokalisationslehre der Gro hirnrinde. Springer, 1909.

[92] Ronald L Paul, Michael Merzenich, and Herbert Goodman. Representation of slowly and rapidly adapting cutaneous mechanoreceptors of the hand in

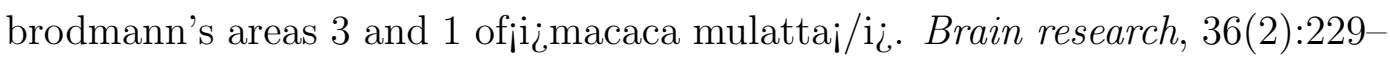
249,1972 .

[93] Hideo Sakata, Yoshio Takaoka, Atsushi Kawarasaki, and Hidetoshi Shibutani. Somatosensory properties of neurons in the superior parietal cortex (area 5) of the rhesus monkey. Brain research, 64:85-102, 1973.

[94] Takashi Hanakawa, Manabu Honda, Nobukatsu Sawamoto, Tomohisa Okada, Yoshiharu Yonekura, Hidena Fukuyama, and Hiroshi Shibasaki. The role of 
rostral brodmann area 6 in mental-operation tasks: an integrative neuroimaging approach. Cerebral Cortex, 12(11):1157-1170, 2002.

[95] Jeffrey L Cummings. Frontal-subcortical circuits and human behavior. Archives of neurology, 50(8):873-880, 1993.

[96] F Ganong William. Fisiología médica. 15a. Edición. Editorial El Manual Moderno, 1996.

[97] Stephen M Kosslyn, A Pascual-Leone, O Felician, S Camposano, JP Keenan, G Ganis, KE Sukel, NM Alpert, et al. The role of area 17 in visual imagery: convergent evidence from pet and rtms. Science, 284(5411):167-170, 1999.

[98] Karl Spencer Lashley. The mechanism of vision: Xviii. effects of destroying the visual. ${ }^{\mathrm{a}}$ Ssociative areas. ${ }^{\circ} \mathrm{f}$ the monkey. Genetic psychology monographs, 1948.

[99] Cathy J Price, Jenny T Crinion, and Mairéad MacSweeney. A generative model of speech production in brocas and wernickes areas. Frontiers in psychology, 2,2011 .

[100] Karl HS Kim, Norman R Relkin, Kyoung-Min Lee, and Joy Hirsch. Distinct cortical areas associated with native and second languages. Nature, 388(6638):171-174, 1997.

[101] Eric C Leuthardt, John Cunningham, and Dennis Barbour. Towards a speech bci using ecog. In Brain-Computer Interface Research, pages 93-110. Springer, 2013.

[102] Anusha Sritharan, Per Line, Alex Sergejew, Richard Silberstein, Gary Egan, and David Copolov. Eeg coherence measures during auditory hallucinations in schizophrenia. Psychiatry research, 136(2):189-200, 2005.

[103] Jie Wang, Dongyu Wu, Yan Chen, Ying Yuan, and Meikui Zhang. Effects of transcranial direct current stimulation on language improvement and cortical activation in nonfluent variant primary progressive aphasia. Neuroscience letters, 549:29-33, 2013. 
[104] Daniel J Levitin and Vinod Menon. Musical structure is processed in language areas of the brain: a possible role for brodmann area 47 in temporal coherence. Neuroimage, 20(4):2142-2152, 2003.

[105] Katrin Amunts, Aleksandar Malikovic, Hartmut Mohlberg, Thorsten Schormann, and Karl Zilles. Brodmann's areas 17 and 18 brought into stereotaxic space - where and how variable? Neuroimage, 11(1):66-84, 2000.

[106] Simon B Eickhoff, Klaas E Stephan, Hartmut Mohlberg, Christian Grefkes, Gereon R Fink, Katrin Amunts, and Karl Zilles. A new spm toolbox for combining probabilistic cytoarchitectonic maps and functional imaging data. Neuroimage, 25(4):1325-1335, 2005.

[107] Giovanni Buccino, Ferdinand Binkofski, Gereon R Fink, Luciano Fadiga, Leonardo Fogassi, Vittorio Gallese, Rüdiger J Seitz, Karl Zilles, Giacomo Rizzolatti, and H-J Freund. Action observation activates premotor and parietal areas in a somatotopic manner: an fmri study. European journal of neuroscience, 13(2):400-404, 2001.

[108] Benedict Shien Wei Ng, Nikos K Logothetis, and Christoph Kayser. Eeg phase patterns reflect the selectivity of neural firing. Cerebral Cortex, 23(2):389-398, 2013.

[109] Quentin Noirhomme, Richard I Kitney, and Benoit Macq. Single-trial eeg source reconstruction for brain-computer interface. Biomedical Engineering, IEEE Transactions on, 55(5):1592-1601, 2008.

[110] AL Hodgkin and AF Huxley. Propagation of electrical signals along giant nerve fibres. Proceedings of the Royal Society of London. Series B, Biological Sciences, pages 177-183, 1952.

[111] Gilberto Flores. Modelación y estudio matemático del proceso de conducción de impulsos eléctricos en nervios. Revista de la Real Academia de Ciencias Exactas, Físicas y Naturales, 87(2):223-262, 1993. 
[112] Fernando Lopes da Silva. Functional localization of brain sources using eeg and/or meg data: volume conductor and source models. Magnetic resonance imaging, 22(10):1533-1538, 2004.

[113] Christophe Phillips, Michael D Rugg, and Karl J Friston. Anatomically informed basis functions for eeg source localization: combining functional and anatomical constraints. NeuroImage, 16(3):678-695, 2002.

[114] Christoph M Michel, Micah M Murray, Göran Lantz, Sara Gonzalez, Laurent Spinelli, and Rolando Grave de Peralta. Eeg source imaging. Clinical neurophysiology, 115(10):2195-2222, 2004.

[115] Rodrigo Sigala, Sebastian Haufe, Dipanjan Roy, Hubert R Dinse, and Petra Ritter. The role of alpha-rhythm states in perceptual learning: insights from experiments and computational models. Frontiers in computational neuroscience, 8, 2014.

[116] Seon Chill Kim, Myoung Hee Lee, Chel Jang, Jung Won Kwon, and Joo Wan Park. The effect of alpha rhythm sleep on eeg activity and individuals attention. Journal of physical therapy science, 25(12):1515, 2013.

[117] Erol Başar, Canan Başar-Eroglu, Sirel Karakaş, and Martin Schürmann. Gamma, alpha, delta, and theta oscillations govern cognitive processes. International Journal of Psychophysiology, 39(2):241-248, 2001.

[118] FH Lopes da Silva and W Storm van Leeuwen. The cortical source of the alpha rhythm. Neuroscience letters, 6(2):237-241, 1977.

[119] Saeid Sanei and Jonathon A Chambers. EEG signal processing. John Wiley \& Sons, 2008.

[120] J Carlos Sierra, A Fernández-Guardiola, G Luna-Villegas, and G Buela-Casal. Efectos residuales de las benzodiacepinas sobre la atención en humanos. Psicothema, 5(2):277-291, 1993.

[121] Andreas K Engel and Pascal Fries. Beta-band oscillations - signalling the status quo? Current opinion in neurobiology, 20(2):156-165, 2010. 
[122] Till R Schneider, Stefan Debener, Robert Oostenveld, and Andreas K Engel. Enhanced eeg gamma-band activity reflects multisensory semantic matching in visual-to-auditory object priming. Neuroimage, 42(3):1244-1254, 2008.

[123] C Mulert, V Kirsch, Roberto Pascual-Marqui, Robert W McCarley, and Kevin M Spencer. Long-range synchrony of gamma oscillations and auditory hallucination symptoms in schizophrenia. International Journal of Psychophysiology, 79(1):55-63, 2011.

[124] Keith B Doelling, Luc H Arnal, Oded Ghitza, and David Poeppel. Acoustic landmarks drive delta-theta oscillations to enable speech comprehension by facilitating perceptual parsing. Neuroimage, 85:761-768, 2014.

[125] Ian G Campbell and Irwin Feinberg. Longitudinal trajectories of non-rapid eye movement delta and theta eeg as indicators of adolescent brain maturation. Proceedings of the National Academy of Sciences, 106(13):5177-5180, 2009.

[126] Jonathan R Wolpaw, Dennis J McFarland, and Theresa M Vaughan. Braincomputer interface research at the wadsworth center. Rehabilitation Engineering, IEEE Transactions on, 8(2):222-226, 2000.

[127] Dennis J McFarland, Dean J Krusienski, and Jonathan R Wolpaw. Braincomputer interface signal processing at the wadsworth center: mu and sensorimotor beta rhythms. Progress in brain research, 159:411-419, 2006.

[128] G Pfurtscheller, GR Müller-Putz, A Schlögl, B Graimann, R Scherer, R Leeb, C Brunner, C Keinrath, F Lee, G Townsend, et al. 15 years of bci research at graz university of technology: current projects. IEEE transactions on neural systems and rehabilitation engineering: a publication of the IEEE Engineering in Medicine and Biology Society, 14(2):205-210, 2006.

[129] Robert Leeb, Doron Friedman, Gernot R Müller-Putz, Reinhold Scherer, Mel Slater, and Gert Pfurtscheller. Self-paced (asynchronous) bci control of a wheelchair in virtual environments: a case study with a tetraplegic. Computational intelligence and neuroscience, 2007, 2007. 
[130] Eric R Kandel, James H Schwartz, Thomas M Jessell, et al. Principles of neural science, volume 4. McGraw-Hill New York, 2000.

[131] Jaime A Pineda. The functional significance of mu rhythms: translating seeing and hearing into doing. Brain Research Reviews, 50(1):57-68, 2005.

[132] G Pfurtscheller, Ch Neuper, C Andrew, and G Edlinger. Foot and hand area mu rhythms. International Journal of Psychophysiology, 26(1-3):121-135, 1997.

[133] Marcel van Gerven, Jason Farquhar, Rebecca Schaefer, Rutger Vlek, Jeroen Geuze, Anton Nijholt, Nick Ramsey, Pim Haselager, Louis Vuurpijl, Stan Gielen, et al. The brain-computer interface cycle. Journal of Neural Engineering, 6(4):041001, 2009.

[134] Fabien Lotte, Marco Congedo, Anatole Lécuyer, Fabrice Lamarche, Bruno Arnaldi, et al. A review of classification algorithms for eeg-based brain-computer interfaces. Journal of neural engineering, 4, 2007.

[135] Gert Pfurtscheller, C Neuper, C Guger, WAHW Harkam, Herbert Ramoser, Alois Schlogl, BAOB Obermaier, MAPM Pregenzer, et al. Current trends in graz brain-computer interface (bci) research. IEEE Transactions on Rehabilitation Engineering, 8(2):216-219, 2000.

[136] Pradeep Shenoy, Matthias Krauledat, Benjamin Blankertz, Rajesh PN Rao, and Klaus-Robert Müller. Towards adaptive classification for bci. Journal of neural engineering, 3(1):R13, 2006.

[137] Steven G Mason and Gary E Birch. A general framework for brain-computer interface design. Neural Systems and Rehabilitation Engineering, IEEE Transactions on, 11(1):70-85, 2003.

[138] Brendan Z Allison, Dennis J McFarland, Gerwin Schalk, Shi Dong Zheng, Melody Moore Jackson, and Jonathan R Wolpaw. Towards an independent brain-computer interface using steady state visual evoked potentials. Clinical Neurophysiology, 119(2):399-408, 2008. 
[139] Jaimie F Borisoff, Steven G Mason, Ali Bashashati, and Gary E Birch. Braincomputer interface design for asynchronous control applications: improvements to the lf-asd asynchronous brain switch. Biomedical Engineering, IEEE Transactions on, 51(6):985-992, 2004.

[140] Gernot R Muller-Putz and Gert Pfurtscheller. Control of an electrical prosthesis with an ssvep-based bci. Biomedical Engineering, IEEE Transactions on, 55(1):361-364, 2008.

[141] Nicola Neumann, Jochen Kaiser, Boris Kotchoubey, Thilo Hinterberger, Niels P Birbaumer, et al. Brain-computer communication: self-regulation of slow cortical potentials for verbal communication. Archives of physical medicine and rehabilitation, 82(11):1533-1539, 2001.

[142] Niels Birbaumer, Thilo Hinterberger, Andrea Kubler, and Nicola Neumann. The thought-translation device (ttd): neurobehavioral mechanisms and clinical outcome. Neural Systems and Rehabilitation Engineering, IEEE Transactions on, 11(2):120-123, 2003.

[143] Niels Birbaumer. Slow cortical potentials: Plasticity, operant control, and behavioral effects. The Neuroscientist, 5(2):74-78, 1999.

[144] Junichi Takahashi, Akira Yasumura, Eiji Nakagawa, and Masumi Inagaki. Changes in negative and positive eeg shifts during slow cortical potential training in children with attention-deficit/hyperactivity disorder: a preliminary investigation. Neuroreport, 25(8):618-624, 2014.

[145] G Pfurtscheller, C Brunner, A Schlögl, and FH Lopes da Silva. Mu rhythm (de) synchronization and eeg single-trial classification of different motor imagery tasks. Neuroimage, 31(1):153-159, 2006.

[146] Fazel Reza. Recent advances in brain-computer interface systems. 2011.

[147] Nikolina Ivica, Marina Titlic, and Sanda Pavelin. P300 wave changes in patients with multiple sclerosis. Acta Informatica Medica, 21(3):205, 2013. 
[148] Lawrence Ashley Farwell and Emanuel Donchin. Talking off the top of your head: toward a mental prosthesis utilizing event-related brain potentials. Electroencephalography and clinical Neurophysiology, 70(6):510-523, 1988.

[149] Gernot R Müller-Putz, Reinhold Scherer, Christian Brauneis, and Gert Pfurtscheller. Steady-state visual evoked potential (ssvep)-based communication: impact of harmonic frequency components. Journal of neural engineering, 2(4):123, 2005.

[150] D Lesenfants, D Habbal, Z Lugo, M Lebeau, P Horki, E Amico, C Pokorny, F Gómez, A Soddu, G Müller-Putz, et al. An independent ssvep-based braincomputer interface in locked-in syndrome. Journal of neural engineering, 11(3):035002, 2014.

[151] Yuan-Pin Lin, Yijun Wang, and Tzyy-Ping Jung. A mobile ssvep-based braincomputer interface for freely moving humans: The robustness of canonical correlation analysis to motion artifacts. In Engineering in Medicine and Biology Society (EMBC), 2013 35th Annual International Conference of the IEEE, pages 1350-1353. IEEE, 2013.

[152] Wei-Peng Teo and Effie Chew. Is motor-imagery brain-computer interface feasible in stroke rehabilitation? PMÉamp;R, 2014.

[153] Dennis J McFarland and Jonathan R Wolpaw. Sensorimotor rhythm-based brain-computer interface (bci): feature selection by regression improves performance. Neural Systems and Rehabilitation Engineering, IEEE Transactions on, 13(3):372-379, 2005.

[154] Guido Dornhege, Benjamin Blankertz, Gabriel Curio, and Klaus-Robert Müller. Combining features for bci. In Advances in Neural Information Processing Systems, pages 1115-1122, 2002.

[155] Isabelle Guyon and André Elisseeff. An introduction to variable and feature selection. The Journal of Machine Learning Research, 3:1157-1182, 2003.

[156] Lei Yu and Huan Liu. Efficient feature selection via analysis of relevance and redundancy. The Journal of Machine Learning Research, 5:1205-1224, 2004. 
[157] Dario Farina, Omar Feix do Nascimento, Marie-Françoise Lucas, and Christian Doncarli. Optimization of wavelets for classification of movement-related cortical potentials generated by variation of force-related parameters. Journal of neuroscience methods, 162(1):357-363, 2007.

[158] Dennis J McFarland, Charles W Anderson, K Muller, Alois Schlogl, and Dean J Krusienski. Bci meeting 2005-workshop on bci signal processing: feature extraction and translation. IEEE transactions on neural systems and rehabilitation engineering, 14(2):135, 2006.

[159] Zhenghua Wu, Yongxiu Lai, Yang Xia, Dan Wu, and Dezhong Yao. Stimulator selection in ssvep-based bci. Medical engineering 83 physics, 30(8):1079-1088, 2008 .

[160] Deon Garrett, David A Peterson, Charles W Anderson, and Michael H Thaut. Comparison of linear, nonlinear, and feature selection methods for eeg signal classification. Neural Systems and Rehabilitation Engineering, IEEE Transactions on, 11(2):141-144, 2003.

[161] Han-Jeong Hwang, Kiwoon Kwon, and Chang-Hwang Im. Neurofeedbackbased motor imagery training for brain-computer interface (bci). Journal of neuroscience methods, 179(1):150-156, 2009.

[162] Michael Schroder, Martin Bogdan, T Hinterberger, and N Birbaumer. Automated eeg feature selection for brain computer interfaces. In Neural Engineering, 2003. Conference Proceedings. First International IEEE EMBS Conference on, pages 626-629. IEEE, 2003.

[163] Mohammad Reza Haji Samadi and Neil Cooke. A novel approach for adaptive eeg artefact rejection and eog gaze estimation. In HCI International 2013Posters Extended Abstracts, pages 603-607. Springer, 2013.

[164] Rifai Chai, Sai Ho Ling, Gregory P Hunter, and Hung T Nguyen. Toward fewer eeg channels and better feature extractor of non-motor imagery mental tasks classification for a wheelchair thought controller. In Engineering in Medicine 
and Biology Society (EMBC), 2012 Annual International Conference of the IEEE, pages 5266-5269. IEEE, 2012.

[165] José del R Millán, Marco Franzé, Josep Mouriño, Febo Cincotti, and Fabio Babiloni. Relevant eeg features for the classification of spontaneous motorrelated tasks. Biological cybernetics, 86(2):89-95, 2002.

[166] Christian Gerloff, Jacob Richard, Jordan Hadley, Andrew E Schulman, Manabu Honda, and Mark Hallett. Functional coupling and regional activation of human cortical motor areas during simple, internally paced and externally paced finger movements. Brain, 121(8):1513-1531, 1998.

[167] Ulrich Hoffmann, Gary Garcia, J Vesin, Karin Diserens, and Touradj Ebrahimi. A boosting approach to p300 detection with application to brain-computer interfaces. In Neural Engineering, 2005. Conference Proceedings. 2nd International IEEE EMBS Conference on, pages 97-100. IEEE, 2005.

[168] Dean J Krusienski, Dennis J McFarland, and Jonathan R Wolpaw. Value of amplitude, phase, and coherence features for a sensorimotor rhythm-based brain-computer interface. Brain research bulletin, 87(1):130-134, 2012.

[169] HA Baseler, EE Sutter, SA Klein, and T Carney. The topography of visual evoked response properties across the visual field. Electroencephalography and clinical neurophysiology, 90(1):65-81, 1994.

[170] DA Jeffreys and JG Axford. Source locations of pattern-specific components of human visual evoked potentials. i. component of striate cortical origin. Experimental Brain Research, 16(1):1-21, 1972.

[171] Mirko Pham, Thilo Hinterberger, Nicola Neumann, Andrea Kübler, Nadine Hofmayer, Anke Grether, Barbara Wilhelm, Jean-Jacques Vatine, and Niels Birbaumer. An auditory brain-computer interface based on the self-regulation of slow cortical potentials. Neurorehabilitation and Neural Repair, 19(3):206218, 2005. 
[172] GR Muller-Putz, Reinhold Scherer, Christa Neuper, and Gert Pfurtscheller. Steady-state somatosensory evoked potentials: suitable brain signals for braincomputer interfaces? Neural Systems and Rehabilitation Engineering, IEEE Transactions on, 14(1):30-37, 2006.

[173] Georg E Fabiani, Dennis J McFarland, Jonathan R Wolpaw, and Gert Pfurtscheller. Conversion of eeg activity into cursor movement by a brain-computer interface (bci). Neural Systems and Rehabilitation Engineering, IEEE Transactions on, 12(3):331-338, 2004.

[174] Miroslav Kubat, Robert C Holte, and Stan Matwin. Machine learning for the detection of oil spills in satellite radar images. Machine learning, 30(2-3):195215, 1998.

[175] Sebastian Zander, Thuy Nguyen, and Grenville Armitage. Automated traffic classification and application identification using machine learning. In Local Computer Networks, 2005. 30th Anniversary. The IEEE Conference on, pages 250-257. IEEE, 2005.

[176] Igor Kononenko. Machine learning for medical diagnosis: history, state of the art and perspective. Artificial Intelligence in medicine, 23(1):89-109, 2001.

[177] Paul Sajda, Adam Gerson, K Muller, Benjamin Blankertz, and Lucas Parra. A data analysis competition to evaluate machine learning algorithms for use in brain-computer interfaces. Neural Systems and Rehabilitation Engineering, IEEE Transactions on, 11(2):184-185, 2003.

[178] Carmen Vidaurre, Claudia Sannelli, Klaus-Robert Müller, and Benjamin Blankertz. Machine-learning-based coadaptive calibration for brain-computer interfaces. Neural computation, 23(3):791-816, 2011.

[179] Cristina García Cambronero and Irene Gómez Moreno. Algoritmos de aprendizaje: knn \& kmeans. Intelgencia en Redes de Comunicación, Universidad Carlos III de Madrid, 2006. 
[180] K Muller, Charles W Anderson, and Gary E Birch. Linear and nonlinear methods for brain-computer interfaces. Neural Systems and Rehabilitation Engineering, IEEE Transactions on, 11(2):165-169, 2003.

[181] R Fisher. Linear discriminant analysis. Ann. Eugenics, 7:179, 1936.

[182] Katerine Díaz Chito et al. Algunas extensiones del método de vectores comunes discriminantes para la clasificación de imágenes. 2011.

[183] Freddy Hernández Barajas and Juan Carlos Correa Morales. Comparación entre tres técnicas de clasificación. Revista Colombiana de Estadística, 32(2):247$265,2009$.

[184] James A Anderson. Separate sample logistic discrimination. Biometrika, 59(1):19-35, 1972.

[185] Douglas M Hawkins and Geoffrey J McLachlan. High-breakdown linear discriminant analysis. Journal of the American Statistical Association, 92(437):136143, 1997.

[186] Keinosuke Fukunaga. Introduction to statistical pattern recognition. Academic press, 1990.

[187] Juwei Lu, Kostas N Plataniotis, and Anastasios N Venetsanopoulos. Regularized discriminant analysis for the small sample size problem in face recognition. Pattern Recognition Letters, 24(16):3079-3087, 2003.

[188] Jerome H Friedman. Regularized discriminant analysis. Journal of the American statistical association, 84(405):165-175, 1989.

[189] Jieping Ye, Tao Xiong, Qi Li, Ravi Janardan, Jinbo Bi, Vladimir Cherkassky, and Chandra Kambhamettu. Efficient model selection for regularized linear discriminant analysis. In Proceedings of the 15th ACM international conference on Information and knowledge management, pages 532-539. ACM, 2006.

[190] Luis Gomez Chova. Pattern Recognition Methods for Crop Classification from Hyperspectral Remote Sensing Images. Universal-Publishers, 2004. 
[191] Jun Shao. Linear model selection by cross-validation. Journal of the American statistical Association, 88(422):486-494, 1993.

[192] Francisco Herrera, Cesar Hervas, José Otero, and Luciano Sánchez. Un estudio empırico preliminar sobre los tests estadısticos más habituales en el aprendizaje automático. Tendencias de la Minerıa de Datos en Espana, Red Espanola de Minerıa de Datos y Aprendizaje (TIC2002-11124-E), pages 403-412, 2004.

[193] Ron Kohavi et al. A study of cross-validation and bootstrap for accuracy estimation and model selection. In IJCAI, volume 14, pages 1137-1145, 1995.

[194] Wikipedia. Validacion cruzada — wikipedia, la enciclopedia libre, 2014. [Internet; descargado 6-enero-2015].

[195] Fabien Lotte, Cuntai Guan, et al. An efficient p300-based brain-computer interface with minimal calibration time. In Assistive Machine Learning for People with Disabilities symposium (NIPS'09 Symposium), 2009.

[196] Leonardo Jiménez Moscovitz and Pervys Rengifo Rengifo. Al interior de una máquina de soporte vectorial. Revista de Ciencias, Facultad de Ciencias Naturales y Exactas, Universidad del Valle, 14:73-78, 2010.

[197] Gustavo A Betancourt. Las máquinas de soporte vectorial (svms). Scientia et Technica, 1(27), 2005.

[198] A Aizerman, Emmanuel M Braverman, and LI Rozoner. Theoretical foundations of the potential function method in pattern recognition learning. Automation and remote control, 25:821-837, 1964.

[199] Mohammadreza Asghari Oskoei, John Q Gan, and Huosheng Hu. Adaptive schemes applied to online svm for bci data classification. In 31st Annual International Conference of the IEEE EMBS, pages 2600-2603, 2009.

[200] Suryannarayana Chandaka, Amitava Chatterjee, and Sugata Munshi. Crosscorrelation aided support vector machine classifier for classification of eeg signals. Expert Systems with Applications, 36(2):1329-1336, 2009. 
[201] N Fatma Guler and Elif Derya Ubeyli. Multiclass support vector machines for eeg-signals classification. Information Technology in Biomedicine, IEEE Transactions on, 11(2):117-126, 2007.

[202] Alois Schlögl, Felix Lee, Horst Bischof, and Gert Pfurtscheller. Characterization of four-class motor imagery eeg data for the bci-competition 2005. Journal of neural engineering, 2(4):L14, 2005.

[203] Leandro Bueno and José Luis Pons. Un sistema de interfaz cerebro ordenador basado en redes neuronales auto-organizadas. XXVIII Jornadas de Automática, 2007, 2007.

[204] José del R Millán and Josep Mouriño. Asynchronous bci and local neural classifiers: an overview of the adaptive brain interface project. Neural Systems and Rehabilitation Engineering, IEEE Transactions on, 11(2):159-161, 2003.

[205] Gustavo Romero, Maribel García Arenas, PA Castillo, and JJ Merelo. Evolutionary design of a brain-computer interface. In Computational Intelligence and Bioinspired Systems, pages 669-676. Springer, 2005.

[206] Emiliano Aldabas-Rubira. Introducción al reconocimiento de patrones mediante redes neuronales. IX Jornades de Conferències dÉnginyeria Electrònica del Campus de Terrassa, Terrassa, España, del 9 al 16 de Diciembre del 2002, 2002 .

[207] MW Gardner and SR Dorling. Artificial neural networks (the multilayer perceptron)-a review of applications in the atmospheric sciences. Atmospheric environment, 32(14-15):2627-2636, 1998.

[208] Raquel Florez López and José Miguel Fernandez Fernandez. Las redes neuronales artificiales. Netbiblo, 2008.

[209] Marvin Minsky, Seymours Papert, and M IoT Perceptrons. Press. Cambridge, Ma, pages 105-110, 1969. 
[210] Sunil Sivadas and Hynek Hermansky. Generalized tandem feature extraction. In Acoustics, Speech, and Signal Processing, 2003. Proceedings.(ICASSP'03). 2003 IEEE International Conference on, volume 1, pages I-56. IEEE, 2003.

[211] Pari Jahankhani, Vassilis Kodogiannis, and Kenneth Revett. Eeg signal classification using wavelet feature extraction and neural networks. In Modern Computing, 2006. JVA'06. IEEE John Vincent Atanasoff 2006 International Symposium on, pages 120-124. IEEE, 2006.

[212] Jose del R Millan, Josep Mourino, Fabio Babiloni, Febo Cincotti, Markus Varsta, and Jukka Heikkonen. Local neural classifier for eeg-based recognition of mental tasks. In Neural Networks, IEEE-INNS-ENNS International Joint Conference on, volume 3, pages 3632-3632. IEEE Computer Society, 2000.

[213] JR del Millán. On the need for on-line learning in brain-computer interfaces. In Neural Networks, 2004. Proceedings. 2004 IEEE International Joint Conference on, volume 4, pages 2877-2882. IEEE, 2004.

[214] Rich Caruana and Alexandru Niculescu-Mizil. An empirical comparison of supervised learning algorithms. In Proceedings of the 23rd international conference on Machine learning, pages 161-168. ACM, 2006.

[215] Gail Carpenter, Stephen Grossberg, et al. A self-organizing neural network for supervised learning, recognition, and prediction. Communications Magazine, IEEE, 30(9):38-49, 1992.

[216] Guzmán Santafé, Jose A Lozano, and Pedro Larrañaga. Aprendizaje discriminativo de clasificadores bayesianos. Inteligencia Artificial. Revista Iberoamericana de Inteligencia Artificial, 10(29):39-47, 2006.

[217] Haddad Haddad, Maher Khatib, Simon Lacroix, and Raja Chatila. Reactive navigation in outdoor environments using potential fields. In Robotics and Automation, 1998. Proceedings. 1998 IEEE International Conference on, volume 2, pages 1232-1237. IEEE, 1998. 
[218] Sasan S Saatchi, Joao Vianei Soares, and Diogenes Salas Alves. Mapping deforestation and land use in amazon rainforest by using sir-c imagery. Remote Sensing of Environment, 59(2):191-202, 1997.

[219] Irina Rish. An empirical study of the naive bayes classifier. In IJCAI 2001 workshop on empirical methods in artificial intelligence, volume 3, pages 41-46, 2001.

[220] Thiago Bassani and Julio Cesar Nievola. Brain-computer interface using wavelet transformation and naive bayes classifier. In Brain Inspired Cognitive Systems 2008, pages 147-165. Springer, 2010.

[221] Gabriela García Manzo and Guillermo De la Torre-Gea. Análisis de la relación amplitud-frecuencia en las señales eeg mediante redes bayesianas. Avances en Tecnologías de Información, page 115.

[222] Gabriel Pires, Miguel Castelo-Branco, and Urbano Nunes. Visual p300-based bci to steer a wheelchair: a bayesian approach. In Engineering in Medicine and Biology Society, 2008. EMBS 2008. 30th Annual International Conference of the IEEE, pages 658-661. IEEE, 2008.

[223] Leonard E Baum, Ted Petrie, George Soules, and Norman Weiss. A maximization technique occurring in the statistical analysis of probabilistic functions of markov chains. The annals of mathematical statistics, pages 164-171, 1970.

[224] William J Stewart. Introduction to the numerical solution of Markov chains, volume 41. Princeton University Press Princeton, 1994.

[225] Miguel Ángel Patiño Antonioli. Aplicación de las cadenas ocultas de markov para la preferencia de los consumidores en el mercado cervecero. 2011.

[226] Xuedong D Huang, Yasuo Ariki, and Mervyn A Jack. Hidden Markov models for speech recognition, volume 2004. Edinburgh university press Edinburgh, 1990. 
[227] Jianying Hu, Michael K Brown, and William Turin. Hmm based online handwriting recognition. Pattern Analysis and Machine Intelligence, IEEE Transactions on, 18(10):1039-1045, 1996.

[228] Hyeon-Kyu Lee and Jin-Hyung Kim. An hmm-based threshold model approach for gesture recognition. Pattern Analysis and Machine Intelligence, IEEE Transactions on, 21(10):961-973, 1999.

[229] B Obermaier, GR Muller, and G Pfurtscheller. "virtual keyboardçontrolled by spontaneous eeg activity. IEEE Transactions on Neural Systems and Rehabilitation Engineering, 11(4):422-426, 2003.

[230] Silvia Chiappa, Nicolas Donckers, Samy Bengio, and Frédéric Vrins. Hmm and iohmm modeling of eeg rhythms for asynchronous bci systems. In ESANN, pages 193-204, 2004.

[231] Yoshua Bengio and Paolo Frasconi. An input output hmm architecture. Advances in neural information processing systems, pages 427-434, 1995.

[232] Bernhard Obermaier, Christoph Guger, Christa Neuper, and Gert Pfurtscheller. Hidden markov models for online classification of single trial eeg data. Pattern recognition letters, 22(12):1299-1309, 2001.

[233] Bernard W Silverman and M Christopher Jones. E. fix and jl hodges (1951): An important contribution to nonparametric discriminant analysis and density estimation: Commentary on fix and hodges (1951). International Statistical Review/Revue Internationale de Statistique, pages 233-238, 1989.

[234] Toyofumi Saito and Jun-Ichiro Toriwaki. New algorithms for euclidean distance transformation of an n-dimensional digitized picture with applications. Pattern recognition, 27(11):1551-1565, 1994.

[236] Nele Verbiest, Chris Cornelis, and Francisco Herrera. Selección de prototipos basada en conjuntos rugosos difusos. In Proceedings of XVI Congreso espanol sobre Tecnologias y Lógica (ESTYLF2012), pages 638-643, 2012. 
[237] Akram Saleh Mousa AlSukker. An Improved EEG Pattern Classification System Based on Dimensionality Reduction and Classifier Fusion. PhD thesis, University of Technology, Sydney, 2012.

[238] Guido Dornhege, Matthias Krauledat, Klaus-Robert Muller, and Benjamin Blankertz. 13 general signal processing and machine learning tools for bci analysis. Toward Brain-Computer Interfacing, page 207, 2007.

[239] Piotr Indyk and Rajeev Motwani. Approximate nearest neighbors: towards removing the curse of dimensionality. In Proceedings of the thirtieth annual ACM symposium on Theory of computing, pages 604-613. ACM, 1998.

[240] Zexuan Zhu, Sen Jia, and Zhen Ji. Towards a memetic feature selection paradigm [application notes]. Computational Intelligence Magazine, IEEE, 5(2):4153, 2010.

[241] E Yorn-Tov and GF Inbar. Selection of relevant features for classification of movements from single movement-related potentials using a genetic algorithm. In Engineering in Medicine and Biology Society, 2001. Proceedings of the 23rd Annual International Conference of the IEEE, volume 2, pages 1364-1366. IEEE, 2001.

[242] Mark JL Orr. Regularization in the selection of radial basis function centers. Neural computation, 7(3):606-623, 1995.

[243] F Nijboer, BZ Allison, S Dunne, D Plass-Oude Bos, A Nijholt, and P Haselager. A preliminary survey on the perception of marketability of brain-computer interfaces and initial development of a repository of bci companies. 2011.

[244] Benjamin Blankertz, K Muller, Gabriel Curio, Theresa M Vaughan, Gerwin Schalk, Jonathan R Wolpaw, Alois Schlogl, Christa Neuper, Gert Pfurtscheller, Thilo Hinterberger, et al. The bci competition 2003: progress and perspectives in detection and discrimination of eeg single trials. Biomedical Engineering, IEEE Transactions on, 51(6):1044-1051, 2004.

[245] Amir Ahangi, Mehdi Karamnejad, Nima Mohammadi, Reza Ebrahimpour, and Nasoor Bagheri. Multiple classifier system for eeg signal classification with 
application to brain-computer interfaces. Neural Computing and Applications, 23(5):1319-1327, 2013.

[246] Alain Rakotomamonjy and Vincent Guigue. Bci competition iii: dataset iiensemble of svms for bci p300 speller. Biomedical Engineering, IEEE Transactions on, 55(3):1147-1154, 2008.

[247] Thomas Weise. Global Optimization Algorithms - Theory and Application. SelfPublished, second edition, June 26, 2009. Online available at http://www.itweise.de/.

[248] Lawrence Davis et al. Handbook of genetic algorithms, volume 115. Van Nostrand Reinhold New York, 1991.

[249] Christopher R Houck, Jeff Joines, and Michael G Kay. A genetic algorithm for function optimization: a matlab implementation. NCSU-IE TR, 95(09), 1995.

[250] John J Grefenstette et al. Genetic algorithms for changing environments. In PPSN, volume 2, pages 137-144, 1992.

[251] John H Holland. Outline for a logical theory of adaptive systems. Journal of the ACM (JACM), 9(3):297-314, 1962.

[252] John H Holland. Adaptation in natural and artificial systems: An introductory analysis with applications to biology, control, and artificial intelligence. $\mathrm{U}$ Michigan Press, 1975.

[253] Jihoon Yang and Vasant Honavar. Feature subset selection using a genetic algorithm. In Feature extraction, construction and selection, pages 117-136. Springer, 1998.

[254] Abdelmalik Moujahid, Iñaki Inza, and Pedro Larrañaga. Tema 2. algoritmos genéticos. Departamento de Ciencias de la Computación e Inteligencia Artificial Universidad del País Vasco, 2008.

[255] Abdolreza Asadi Ghanbari, Ali Broum, Hamidreza Navidi, and Ali Ahmadi. Brain computer interface with genetic algorithm. International Journal of Information, 2(1), 2012. 
[256] Daniel S Weile and Eric Michielssen. Genetic algorithm optimization applied to electromagnetics: A review. Antennas and Propagation, IEEE Transactions on, 45(3):343-353, 1997.

[257] DM Deaven and KM Ho. Molecular geometry optimization with a genetic algorithm. Physical Review Letters, 75(2):288, 1995.

[258] Bernardo Dal Seno, Matteo Matteucci, and Luca Mainardi. Online detection of p300 and error potentials in a bci speller. Computational intelligence and neuroscience, 2010:11, 2010.

[259] Luca Citi, Riccardo Poli, Caterina Cinel, and Francisco Sepulveda. Feature selection and classification in brain computer interfaces by a genetic algorithm. In Late-breaking papers of the Genetic and Evolutionary Computation Conference (GECCO-2004), volume 400, 2004.

[260] Mehrdad Fatourechi, Ali Bashashati, Rabab K Ward, and Gary E Birch. A hybrid genetic algorithm approach for improving the performance of the lf-asd brain computer interface. In Acoustics, Speech, and Signal Processing, 2005. Proceedings.(ICASSP'05). IEEE International Conference on, volume 5, pages v-345. IEEE, 2005.

[261] Myeung-Sook Yoh, Joonho Kwon, and Sunghoon Kim. Neurowander: a bci game in the form of interactive fairy tale. In Proceedings of the 12th ACM international conference adjunct papers on Ubiquitous computing-Adjunct, pages 389-390. ACM, 2010.

[262] Anton Nijholt, Danny Plass-Oude Bos, and Boris Reuderink. Turning shortcomings into challenges: Brain-computer interfaces for games. Entertainment Computing, 1(2):85-94, 2009.

[263] Paul S Hammon, Scott Makeig, Howard Poizner, Emanuel Todorov, and Virginia R De Sa. Predicting reaching targets from human eeg. Signal Processing Magazine, IEEE, 25(1):69-77, 2008. 
[264] Stephan Waldert, Hubert Preissl, Evariste Demandt, Christoph Braun, Niels Birbaumer, Ad Aertsen, and Carsten Mehring. Hand movement direction decoded from meg and eeg. The Journal of neuroscience, 28(4):1000-1008, 2008.

[265] Trent J Bradberry, Rodolphe J Gentili, and José L Contreras-Vidal. Reconstructing three-dimensional hand movements from noninvasive electroencephalographic signals. The journal of Neuroscience, 30(9):3432-3437, 2010.

[266] Trent J Bradberry, Rodolphe J Gentili, and José L Contreras-Vidal. Fast attainment of computer cursor control with noninvasively acquired brain signals. Journal of neural engineering, 8(3):036010, 2011.

[267] Alessandro Presacco, Larry Forrester, and Jose L Contreras-Vidal. Towards a non-invasive brain-machine interface system to restore gait function in humans. In Engineering in Medicine and Biology Society, EMBC, 2011 Annual International Conference of the IEEE, pages 4588-4591. IEEE, 2011.

[268] Dennis J McFarland, William A Sarnacki, and Jonathan R Wolpaw. Electroencephalographic (eeg) control of three-dimensional movement. Journal of Neural Engineering, 7(3):036007, 2010.

[269] Stephan Waldert, Tobias Pistohl, Christoph Braun, Tonio Ball, Ad Aertsen, and Carsten Mehring. A review on directional information in neural signals for brain-machine interfaces. Journal of Physiology-Paris, 103(3):244-254, 2009.

[270] Teresa Talamillo García. Manual básico para enfermeros en electroencefalografía. Enfermería Docente, 94:29-33, 2011.

[271] Maurício Campelo Tavares, M Eng, and Eng Biomédica. Eeg e potenciais evocados-uma introdução. Contronic Sistemas Automáticos Ltda, 2011.

[272] Jieping Ye and Tao Xiong. Computational and theoretical analysis of null space and orthogonal linear discriminant analysis. The Journal of Machine Learning Research, 7:1183-1204, 2006. 
[273] Shuiwang Ji and Jieping Ye. Generalized linear discriminant analysis: a unified framework and efficient model selection. Neural Networks, IEEE Transactions on, 19(10):1768-1782, 2008.

[274] Cezary Z Janikow and Zbigniew Michalewicz. An experimental comparison of binary and floating point representations in genetic algorithms. In ICGA, pages 31-36, 1991.

[275] Chunying Fang, Haifeng Li, and Lin Ma. Eeg signal classification using the event-related coherence and genetic algorithm. In Advances in Brain Inspired Cognitive Systems, pages 92-100. Springer, 2013.

[276] Foster Provost and Ron Kohavi. Guest editorsíntroduction: On applied research in machine learning. Machine learning, 30(2):127-132, 1998.

[277] Tonio Ball, Andreas Schulze-Bonhage, Ad Aertsen, and Carsten Mehring. Differential representation of arm movement direction in relation to cortical anatomy and function. Journal of neural engineering, 6(1):016006, 2009.

[278] Eileen Lew, Ricardo Chavarriaga, Stefano Silvoni, and José del R Millán. Detection of self-paced reaching movement intention from eeg signals. Front. Neuroeng, 5(13), 2012.

[279] Jeong-Seok Woo, Klaus-Robert Muller, and Seong-Whan Lee. Classifying directions in continuous arm movement from eeg signals. In Brain-Computer Interface (BCI), 2015 3rd International Winter Conference on, pages 1-2. IEEE, 2015.

[280] Bin Xia, Oladazimi Maysam, Sandra Veser, Lei Cao, Jie Li, Jie Jia, Hong Xie, and Niels Birbaumer. A combination strategy based brain-computer interface for two-dimensional movement control. Journal of neural engineering, 12(4):046021, 2015.

[281] Soumyadipta Acharya, Matthew S Fifer, Heather L Benz, Nathan E Crone, and Nitish V Thakor. Electrocorticographic amplitude predicts finger positions during slow grasping motions of the hand. Journal of neural engineering, $7(4): 046002,2010$. 
[282] Bradley Voytek, Lavi Secundo, Aurelie Bidet-Caulet, Donatella Scabini, Shirley I Stiver, Alisa D Gean, Geoffrey T Manley, and Robert T Knight. Hemicraniectomy: a new model for human electrophysiology with high spatio-temporal resolution. Journal of cognitive neuroscience, 22(11):2491-2502, 2010.

[283] Carsten Mehring, Jörn Rickert, Eilon Vaadia, Simone Cardoso de Oliveira, Ad Aertsen, and Stefan Rotter. Inference of hand movements from local field potentials in monkey motor cortex. Nature neuroscience, 6(12):1253-1254, 2003.

[284] Eric C Leuthardt, Kai J Miller, Gerwin Schalk, Rajesh PN Rao, and Jeffrey G Ojemann. Electrocorticography-based brain computer interface-the seattle experience. Neural Systems and Rehabilitation Engineering, IEEE Transactions on, 14(2):194-198, 2006.

[285] Leigh R Hochberg, Daniel Bacher, Beata Jarosiewicz, Nicolas Y Masse, John D Simeral, Joern Vogel, Sami Haddadin, Jie Liu, Sydney S Cash, Patrick van der Smagt, et al. Reach and grasp by people with tetraplegia using a neurally controlled robotic arm. Nature, 485(7398):372-375, 2012.

[286] Nuri F Ince, Rahul Gupta, Sami Arica, Ahmed H Tewfik, James Ashe, and Giuseppe Pellizzer. High accuracy decoding of movement target direction in non-human primates based on common spatial patterns of local field potentials. PLoS One, 5(12):e14384, 2010.

[287] Jun Zhuang, Wilson Truccolo, Carlos Vargas-Irwin, and John P Donoghue. Decoding 3-d reach and grasp kinematics from high-frequency local field potentials in primate primary motor cortex. Biomedical Engineering, IEEE Transactions on, 57(7):1774-1784, 2010.

[288] Juan L Pedreño-Molina, Antonio Guerrero-González, Oscar A Florez-Giraldo, and Javier Molina-Vilaplana. Sensory-motor control scheme based on kohonen maps and avite model. In Artificial Neural Nets Problem Solving Methods, pages 185-192. Springer, 2003. 
[289] Meel Velliste, Sagi Perel, M Chance Spalding, Andrew S Whitford, and Andrew B Schwartz. Cortical control of a prosthetic arm for self-feeding. Nature, 453(7198):1098-1101, 2008.

[290] Eliana Costa e Silva, M Fernanda Costa, Wolfram Erlhagen, and Estela Bicho. Global vs. local nonlinear optimization techniques for human-like movement of an anthropomorphic robot. In PROCEEDINGS OF THE INTERNATIONAL CONFERENCE ON NUMERICAL ANALYSIS AND APPLIED MATHEMATICS 2014 (ICNAAM-2014), volume 1648, page 140004. AIP Publishing, 2015.

[291] Yuancan Huang, Jian Li, Qiang Huang, and Philippe Soueres. Anthropomorphic robotic arm with integrated elastic joints for tcm remedial massage. Robotica, 33(02):348-365, 2015.

[292] Douglas A Perednia and Ace Allen. Telemedicine technology and clinical applications. Jama, 273(6):483-488, 1995.

[293] Zhibin Song and Shuxiang Guo. Design process of exoskeleton rehabilitation device and implementation of bilateral upper limb motor movement. Journal of Medical and Biological Engineering, 32(5):323-330, 2012.

[294] Christopher N Schabowsky, Sasha B Godfrey, Rahsaan J Holley, Peter S Lum, et al. Development and pilot testing of hexorr: hand exoskeleton rehabilitation robot. J Neuroeng Rehabil, 7(36):1-16, 2010.

[295] Carmen Vidaurre, A Schlogl, Rafael Cabeza, Reinhold Scherer, and Gert Pfurtscheller. A fully on-line adaptive bci. Biomedical Engineering, IEEE Transactions on, 53(6):1214-1219, 2006.

[296] Gert Pfurtscheller, Gernot R Muller-Putz, Reinhold Scherer, and Christa Neuper. Rehabilitation with brain-computer interface systems. Computer, 41(10):58-65, 2008.

[297] Lun-De Liao, Chi-Yu Chen, I-Jan Wang, Sheng-Fu Chen, Shih-Yu Li, Bo-Wei Chen, Jyh-Yeong Chang, and Chin-Teng Lin. Gaming control using a wearable and wireless eeg-based brain-computer interface device with novel dry foambased sensors. Journal of neuroengineering and rehabilitation, 9(1):5, 2012. 
[298] Jonathan Mercier-Ganady, Fabien Lotte, Emilie Loup-Escande, Maud Marchal, and Anatole Lecuyer. The mind-mirror: See your brain in action in your head using eeg and augmented reality. In Virtual Reality (VR), 2014 iEEE, pages 33-38. IEEE, 2014.

[299] http://www.gtec.at/Products/Hardware-and-Accessories/g.MOBIlab-Specs-Feature

[300] https://emotiv.com/epoc.php.

[301] http://store.neurosky.com/products/mindwave-1.

[302] Andrew Campbell, Tanzeem Choudhury, Shaohan Hu, Hong Lu, Matthew K Mukerjee, Mashfiqui Rabbi, and Rajeev DS Raizada. Neurophone: brainmobile phone interface using a wireless eeg headset. In Proceedings of the second ACM SIGCOMM workshop on Networking, systems, and applications on mobile handhelds, pages 3-8. ACM, 2010.

[303] Kristin Bergman. Cyborgs in the courtroom: The use of google glass recordings in litigation. Rich. JL $\&$ Tech., 20:11-11, 2014.

[304] Matthew Whitaker and Esther Kuku. Google glass: the future for surgical training? Bulletin of The Royal College of Surgeons of England, 96(7):223223, 2014.

[305] Rafsan Halim. Unusual utilisation of mobile technology in medicine. Journal of Mobile Technology in Medicine, 2(2):1-2, 2013.

[306] Juan Esteban Arango, Julián Cárdenas Mazo, and Alejandro Peña Palacio. Sistema para rehabilitación del síndrome del miembro fantasma utilizando interfaz cerebro-computador y realidad aumentada. RISTI-Revista Ibérica de Sistemas e Tecnologias de Informação, (11):93-106, 2013.

[307] Katie Crowley, Aidan Sliney, Ian Pitt, and Dave Murphy. Capturing and using emotion-based bci signals in experiments: how subject's effort can influence results. In Proceedings of the 25th BCS Conference on Human-Computer Interaction, pages 132-138. British Computer Society, 2011. 
[308] Biao Zhang, Jianjun Wang, and Thomas Fuhlbrigge. A review of the commercial brain-computer interface technology from perspective of industrial robotics. In Automation and Logistics (ICAL), 2010 IEEE International Conference on, pages 379-384. IEEE, 2010.

[309] Arzu Guneysu and H Levent Akin. An ssvep based bci to control a humanoid robot by using portable eeg device. In Engineering in Medicine and Biology Society (EMBC), 2013 35th Annual International Conference of the IEEE, pages 6905-6908. IEEE, 2013.

[310] Nicholas A Badcock, Petroula Mousikou, Yatin Mahajan, Peter de Lissa, Johnson Thie, and Genevieve McArthur. Validation of the emotiv epoc® eeg gaming system for measuring research quality auditory erps. PeerJ, 1:e38, 2013.

[311] Yue Liu, Xiao Jiang, Teng Cao, Feng Wan, Peng Un Mak, Pui-In Mak, and Mang I Vai. Implementation of ssvep based bci with emotiv epoc. In Virtual Environments Human-Computer Interfaces and Measurement Systems (VECIMS), 2012 IEEE International Conference on, pages 34-37. IEEE, 2012.

[312] Matthieu Duvinage, Thierry Castermans, Thierry Dutoit, M Petieau, T Hoellinger, C De Saedeleer, K Seetharaman, and G Cheron. A p300-based quantitative comparison between the emotiv epoc headset and a medical eeg device. Biomedical Engineering, 765:2012-764, 2012. 